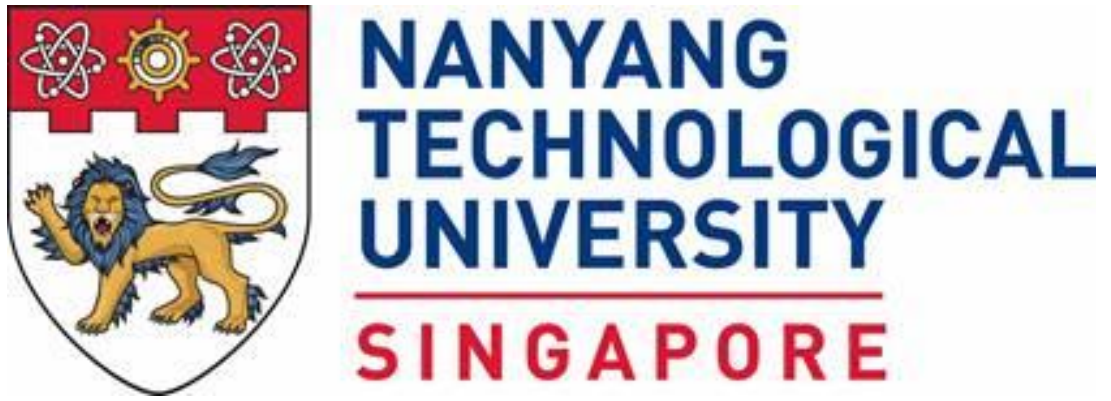

\title{
INVESTIGATIONS INTO SWEPT SOURCE BASED OPTICAL COHERENCE TOMOGRAPHY FOR BIO-IMAGING
}




\section{INVESTIGATIONS INTO SWEPT SOURCE BASED OPTICAL COHERENCE TOMOGRAPHY FOR BIO-IMAGING}

\section{RATHEESH KUMAR MELEPPAT}

School of Mechanical and Aerospace Engineering

A thesis submitted to the Nanyang Technological University in partial fulfilment of the requirement for the degree of Doctor of Philosophy 


\section{ACKNOWLEDGEMENTS}

I take this opportunity to acknowledge the help and encouragement received from various sources during the period my research. I would like to express sincere thanks to my supervisors, Assoc Prof. Seah Leong Keey and Assoc Prof. Murukeshan Vadakke Matham, for providing me the resources and valuable guidance in conducting this thesis. They were continuously inspired me and showed me the right direction to get things on track.

I acknowledge Assoc Prof Christopher Shearwood for the collaborative works conducted in his lab. I also express my heartfelt thanks to all my colleagues and staff of Centre for Optical and Laser Engineering, and Biological Processing Lab for their help, co-operation and support.

I acknowledge the Research Student Scholarship (RSS) and other financial support received through NTU. Also, I acknowledge the funding support received through COLEEDB and A* STAR-SERC towards the completion of this thesis.

I acknowledge Prof Tan Ooi Kiang [Deputy Associate Provost (Undergraduate Education), NTU and SFiR (Crescent Hall)], and Prof NG Pak Tee [National Institute of Education and FiR (Crescent Hall)] for selecting me as a Residential Mentor and provide in-campus accommodation.

I am deeply indebted to my family, especially my wife Karuna and my son Kiran, without their support and encouragement none of this would have been possible. I also would like to thank all my great teachers from public school, who always encouraged me to follow my dreams. Above all, I thank God almighty for guiding and strengthening me these years. 


\section{TABLE OF CONTENTS}

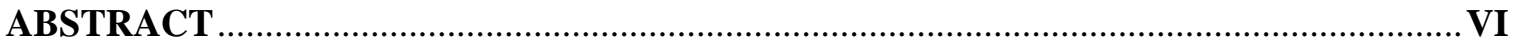

LIST OF FIGURES

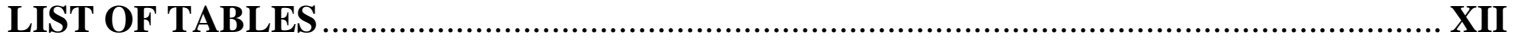

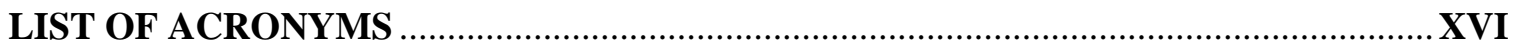

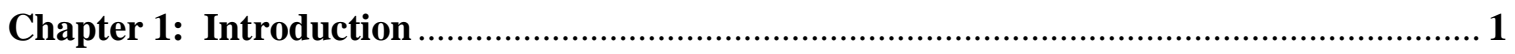

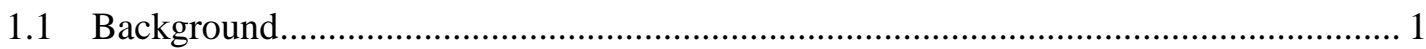

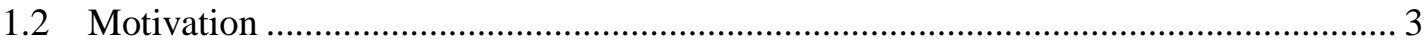

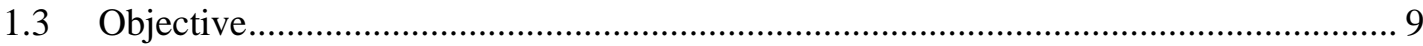

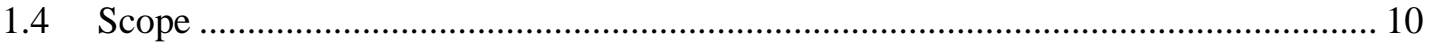

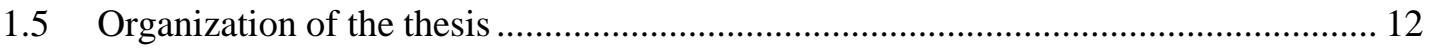

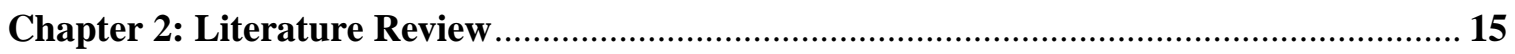

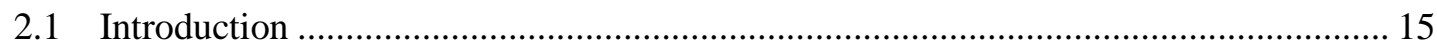

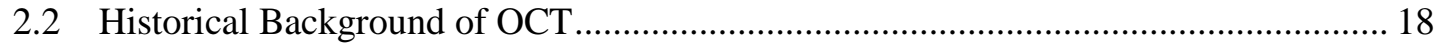

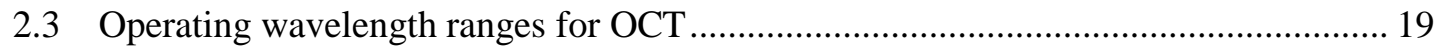

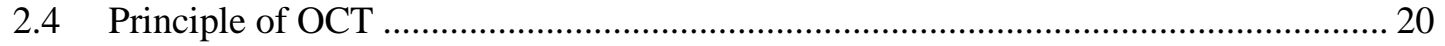

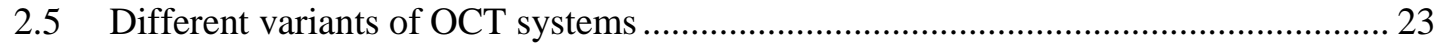

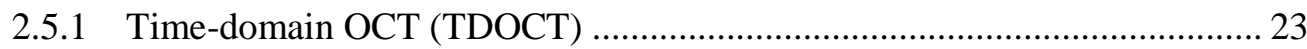

2.5.2 Fourier-domain OCT (FDOCT) ….......................................................... 24

2.6 Different fiber-optic implementations of OCT systems .......................................... 27

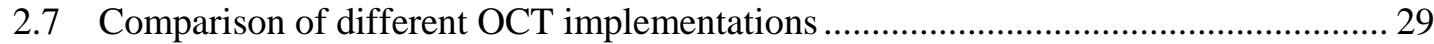

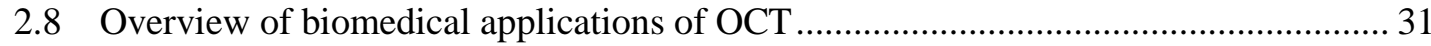

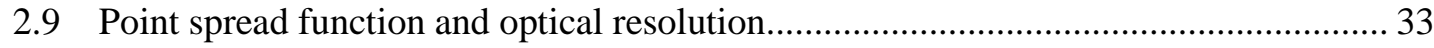

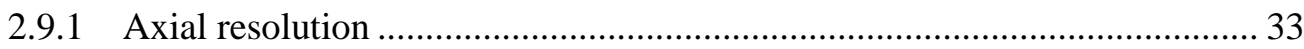

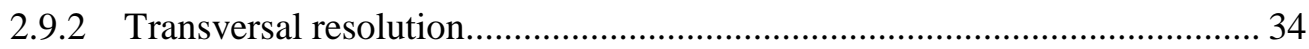

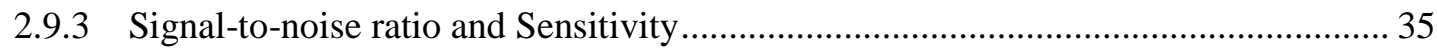


2.10 Comparison of the sensitivities of different OCT configurations.............................. 36

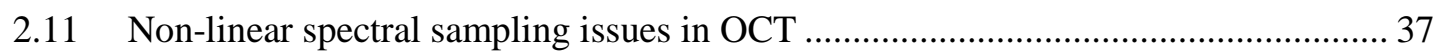

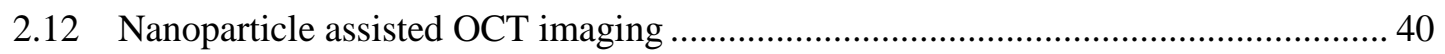

2.13 Optical imaging for real-time monitoring of developmental biology....................... 44

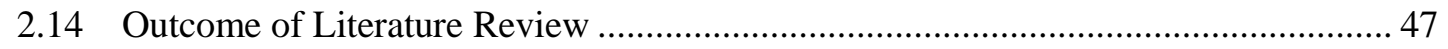

Chapter 3: A compact SSOCT system with enhanced imaging capability ...........................50

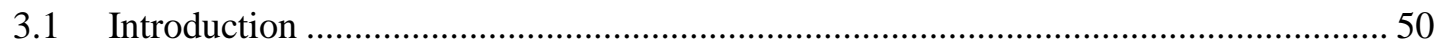

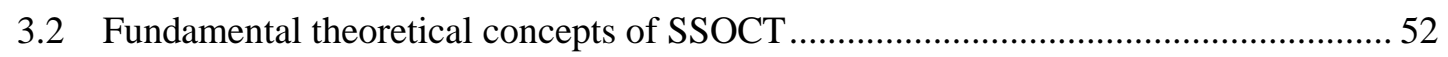

3.3 Instrumentation scheme for the proposed SSOCT system .................................... 55

3.3.1 Frequency-Swept Laser Source ......................................................... 56

3.3.2 Non-reflective reference arm-based Interferometer configuration ............... 57

3.3.3 Balanced detection and data acquisition..................................................5 58

3.3.4 Beam delivery system and control signal generation ...............................59

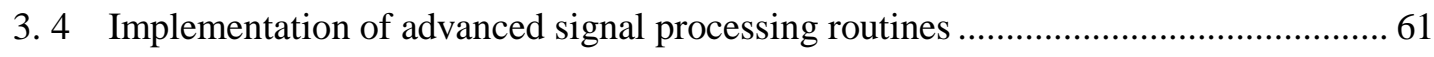

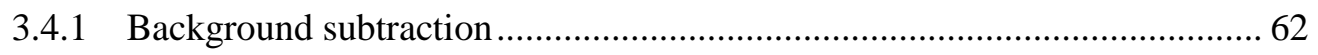

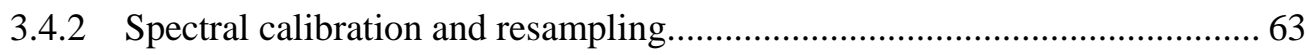

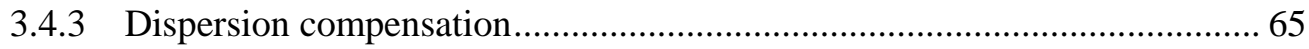

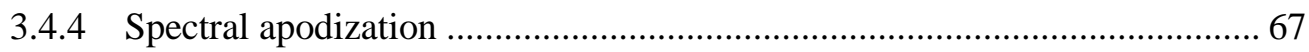

3.4.5 Fourier transform and image construction.............................................. 68

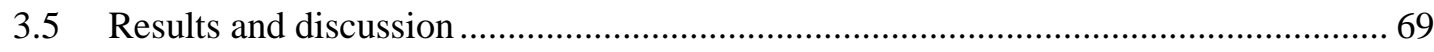

3.5.1 Characterization of the proposed SSOCT system.................................... 69

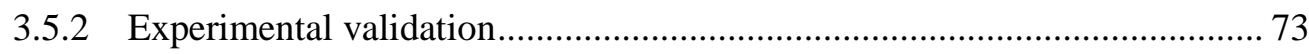

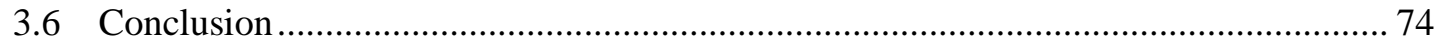

Chapter 4: Real-time automatic spectral calibration for SSOCT systems ........................ 76

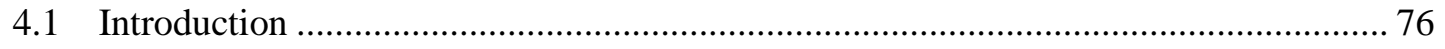

4.2 Implementation of proposed automatic calibration scheme ..................................... 78

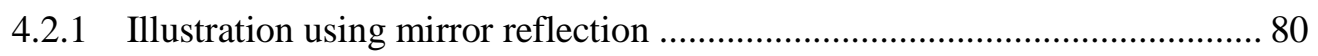




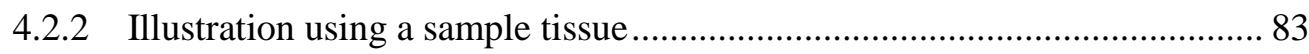

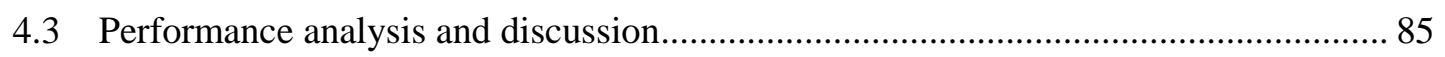

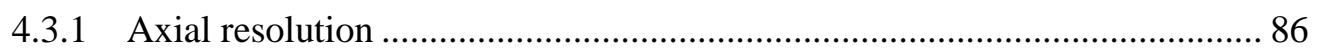

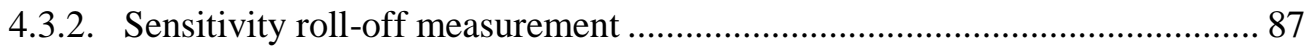

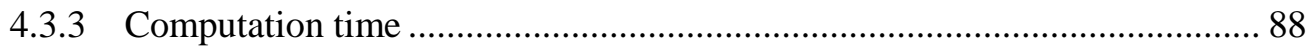

4.3.4 Images acquired with different calibration schemes.................................. 89

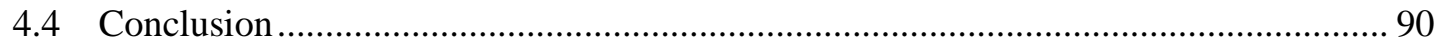

\section{Chapter 5: Nanoparticle-Assisted Contrast Enhancement in Optical Coherence}

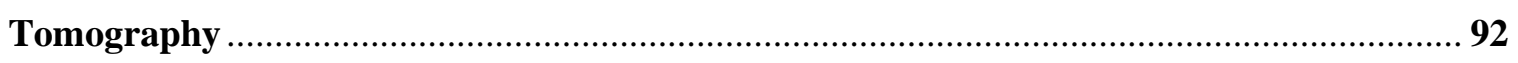

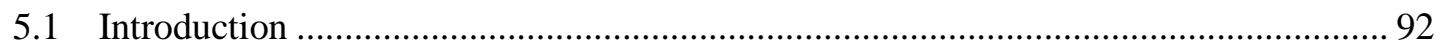

5.1.1 Surface plasmon resonance ................................................................. 94

5.1.2 Surface plasmon absorption, scattering and optical tuning ........................ 95

5.1.3 Modelling and simulation using finite difference time domain method.......96

5.2 Investigation into contrast enhancement using Silica-Coated Silver Nanoplates......... 97

5.2.1 FDTD model for LSPR Characterization in Silica-Coated Silver Nanoplates98

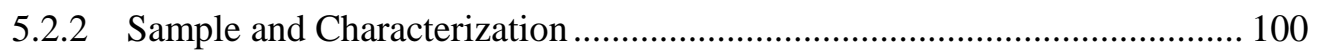

5.2.3 LSPR Characterization using OCT..................................................... 101

5.2.4 Experimental investigation into contrast enhancement using SSNPs ........ 104

5.2.5 Speckle-variance imaging for the analysis of the nanoparticles distribution 106

5.3 Gold nanorods with higher aspect-ratio as absorption-based contrast agent for OCT 108

5.3.1 FDTD model for LSPR characterization in gold nanorod ......................... 108

5.3.2 Sample and Characterization ......................................................... 110

5.3.3 Estimation of extinction cross-section using OCT .................................. 111

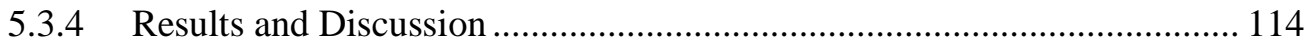

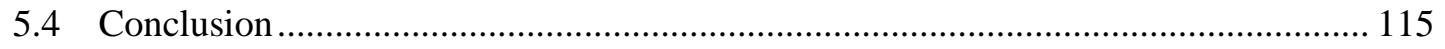

Chapter 6: Real-time in-situ investigation of bacterial biofilms using optical coherence

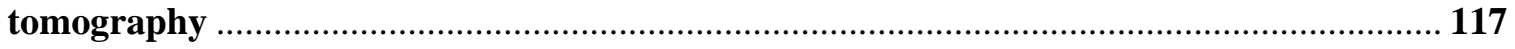

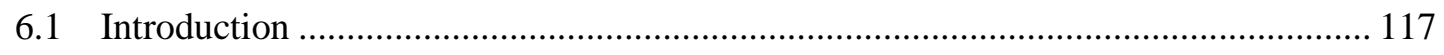


6.2 In-situ investigation of biofilms of Pseudomonas Aeruginosa ............................... 119

6.2.1 Experiments and operational conditions................................................ 120

6.2.2 Methodology for the simultaneous measurement of refractive index and thickness of biofilm ........................................................................ 121

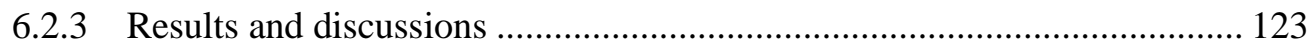

6.3 Real-time in-situ investigation of Klebsiella Pneumonia using SSOCM ................... 124

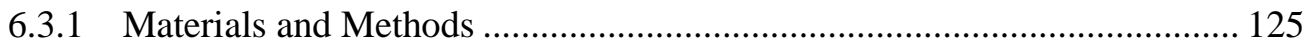

6.3.2 Real-time optical density measurement using OCM technique.................. 128

6.3.3 Measurement of planar density measurement ....................................... 129

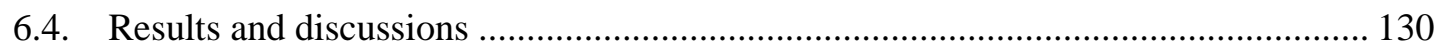

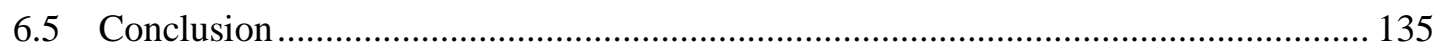

Chapter 7: Conclusions and Future Research Directions ............................................. 137

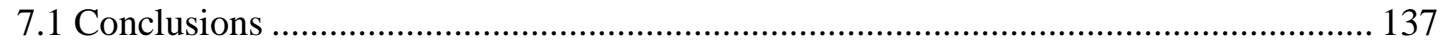

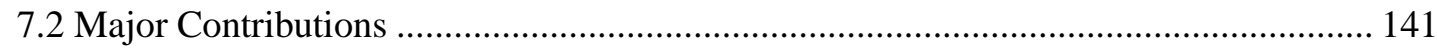

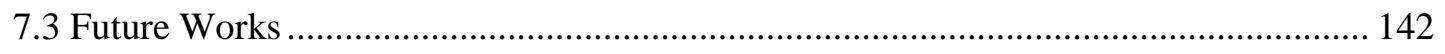

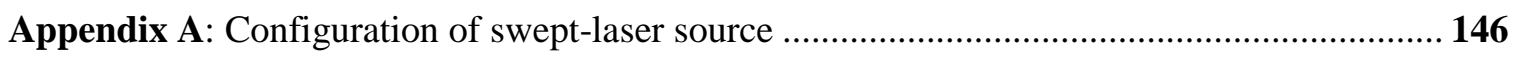

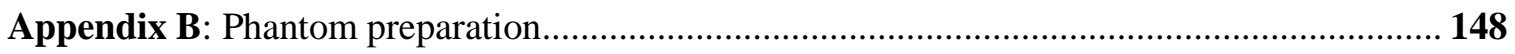

Appendix C: Qualitative Investigation of photothermal effect of gold nanorods....................... 151

Appendix D: Experimental arrangement for the real-time in-situ investigation of biofilms ...... 153

Appendix E: Enface images of Klebsiella Pneumonia .......................................................... 154

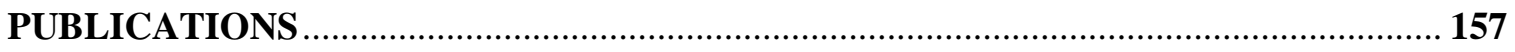

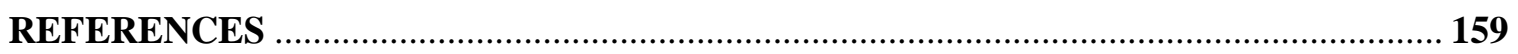




\begin{abstract}
Optical imaging at both the macroscopic and microscopic levels is used intensively these days by clinicians for diagnosis and treatment. New advances in optics, data acquisition, and signal processing enabled the development of novel optical imaging technologies with enhanced imaging capabilities for targeted applications. Further, optical imaging technologies are more affordable than conventional radiation based technologies and provide both structural and functional information with enhanced resolution. Among different optical imaging modalities, optical coherence tomography (OCT) has gained a special attraction since it allows a non-invasive and in-vivo subsurface imaging of the biological specimen at high resolution and relatively better penetration depth. Fourierdomain OCT (FDOCT) configurations are highly attractive for many diagnostic imaging applications due to their potential for high speed imaging with high sensitivity. Nevertheless, the imaging capability and applicability of OCT are often challenged by the factors such as complex and delicate system design, degradation of axial resolution and sensitivity, and reduced contrast. Accordingly, intense efforts aimed at making technical improvements in resolution, image quality, and functional capabilities are underway among the scientific community.
\end{abstract}

A compact FDOCT system based on swept-source configuration (SSOCT) with enhanced imaging capabilities has been proposed and demonstrated in this thesis. An interferometry system based on the non-reflective reference arm has been introduced in place of the Michelson interferometer to offer highly stable, alignment free and portable architecture. The degradation of the axial resolution and sensitivity caused by the non-linear sweeping characteristics of the laser and optical dispersion are efficiently corrected by a spectral 
phase-based wavenumber calibration and a numerical dispersion correction scheme, respectively. The proposed calibration scheme is implemented by an automatic method, which further reduces the computational and hardware complexity. Performance of the system is analyzed experimentally and compared with their theoretically predicted values.

Contrast enhancement in OCT imaging using plasmonic nanoprobes has been investigated. Plasmon-resonant nanostructures of gold and silver have been demonstrated as powerful contrast agents for OCT imaging around $1300 \mathrm{~nm}$. The localized surface plasmon response (LSPR) induced extinction properties of the nanoparticles have been characterized by OCT using a cross-correlation approach. The extinction cross-section measured using OCT found to be consistent with the simulation model based on finite difference time domain (FDTD) and spectroscopy method. The qualitative and quantitative investigations of the contrast enhancement using proposed nanoprobes have been conducted on both biological tissue as well as tissue mimicking phantom.

The project further aims in exploring the potential of optical coherence tomography and microscopy for the real-time in-situ investigation of bacterial biofilms. Both the crosssectional and enface images are presented to study the development of biofilms and their time-resolved growth. The simultaneous measurement of refractive index and the thickness of the biofilms (Pseudomonas Aeruginosa) using OCT are demonstrated. Monitoring of growth dynamics of biofilm (Klebsiella Pneumonia) using optical density (OD) and planar density are also demonstrated using optical coherence microscopy technique and compared with OD and colony forming units (CFUs) measured using standard procedures. It is envisaged that the outcome of the research presented in this thesis will contribute well towards the diagnostic abilities of the OCT based bio imaging systems. 


\section{LIST OF FIGURES}

Figure 1.1 Block diagram representation of the whole research methodology implemented for the research thesis.

Figure 2.1 Performance of different biomedical imaging techniques with respect to image penetration and achievable resolution (Logarithmic scale).

Figure 2.2 Schematic of Michelson Interferometer used in OCT .20

Figure 2.3 Interferogram obtained for (a) Single reflector (b) Multiple reflectors .22

Figure 2.4 Schematic of Time-domain OCT system. 23

Figure 2.5 Schematic of spectral-domain OCT 25

Figure 2.6 Schematic of swept source based-OCT system 27

Figure 2.7 Schematic of a typical fiber-optic SSOCT system with MI configuration. L1-L4, lenses; M1, mirror; OB, water-immersion objective; D1-D2, dual-balanced photodetectors; FW, neutral density filter wheel; DC, dispersion compensating glass.

Figure 2.8. Schematic of SSOCT system with MEMS based common-path endoscopic imaging probe.

Figure 2.9 Fixed fiber Fabry-Perot interferometer (FFPI) and photodetector (PD) based trigger pulse generation scheme for uniform k-space sampling. .38

Figure 3.1 Modular structure of a SSOCT system. .51

Figure 3.2 Signal reconstruction in SSOCT systems .53

Figure 3.3 Representative figure showing the decay of the amplitude of the PSF .54

Figure 3.4 (a) Schematic of the developed SSOCT system (b) Photograph of the developed system. BD: Balance detector; C: Collimator; DAQ: Data acquisition and Control; FC: Fiber coupler; GM: Galvo Mirror; ILA: In-line variable Attenuator; PC: Personal Computer; PD: Photodetector. .56

Figure 3.5 Spectrum of the SL1325-P16 swept laser acquired using optical spectrum analyzer.........57

Figure 3.6 (a) Schematic of sample arm optics (a) Photograph of the sample arm optics used in the system.

Figure 3.7 Control waveform for galvo mirror based beam steering. AB: forward scan BC: backward scan .

Figure 3.8 Process flow of signal processing steps in SSOCT .62

Figure 3.9 Spectral waveform (a) before background subtraction, and (b) after background subtraction. 
Figure 3.10 (a) and (b) shows spectral waveform and PSF before sampling and (b) and (d), after sampling.

Figure 3.11 Illustration of dispersion (a) Spectral waveform with (red) and without dispersion (blue) (b) unwrapped phase plot (c) PSF before (blue) and after dispersion correction (red) (d) zoomed image of selected portion.

Figure 3.12 Spectral apodization using Hamming window (a) before apodization, and (b) after apodization. (c) PSF obtained before (blue) and after apodization (red). .68

Figure 3.13 Enface image of the USAF chart demonstrating the lateral resolution of the imaging system.

Figure 3.14 Gaussian approximated point spread function ......................................................... 70

Figure 3.15 (a) PSF at different depth positions, and (b) sensitivity fall-off as function of depth.......72

Figure 3.16 Images acquired by SSOCT system (a) finger nail-skin region (b) iridocorneal angle of pig eye. (Images are resized for better visual representation). 74

Figure 4.1 Block schematic of typical calibration setup in SSOCT systems .77

Figure 4.2 Block schematic of the proposed real time automatic calibration scheme 79

Figure 4.3 (a) Fringe signal before noise removal (b) fringe signal after noise removal (c) and (d) zoomed view of raw fringe signal before and after noise removal (e) and (f) FFT of fringe signal before and after noise removal.

Figure 4.4 (a) Unwrapped phase function (b) resampling function (c) resampled spectral waveform

(d) Fourier transform of the resampled spectral waveform data.

Figure 4.5 (a) Phase functions acquired from different depths, and (b) Resampling functions corresponding to the individual phase functions.

Figure 4.6 (a) Cross-sectional image of the finger nail-skin sample before calibration (b) A-scan acquired from an arbitrary position (c) Band-pass filtered signal representing the surface reflection (d) (d) Spectral phase function (e) Resampling function (f) Resampled 84

Figure 4.7 (a) PSF for the proposed scheme and PTDI with $6^{\text {th }}$ order polynomial fitting (b) FWHM at different depths (c) PSF for proposed scheme and without calibration scheme at $1.5 \mathrm{~mm}$ (d) PSF obtained for proposed scheme, PTDI with $6^{\text {th }}$ order and NNC scheme

Figure 4.8 Sensitivity roll-off measurement of different calibration schemes (a) absolute values (b) normalized sensitivity roll-off curve.

Figure 4.9 Images obtained under different calibration schemes. (a)-(d) Images of human finger nail and skin region; (e)-(h) images of an anterior chamber eye model; (a) and (e) without any calibration scheme; (b) and (f) using NNC; (c) and (g) using PTDI (sixth-order fitting); (d) and (h) using the proposed linearization scheme. .90 
Figure 5.1 Reflection from the boundary between two media

Figure 5.2 Variation of the resonance peak wavelength with (a) side length (b) thickness of silicacoating (c) enhancement of the electric field in the triangular-shaped SSNP. (b) extinction, scattering and absorption cross-section plot using the FDTD method.

Figure 5.3 (a) TEM image of SSNPs (b) UV-Vis-NIR extinction spectrum recorded for SSNPs with a concentration of $1 \mathrm{mg} / \mathrm{mL}$. 101

Figure 5.4 (a) Cross-sectional image of SSNPs filled in a flow channel. (b) Confocal point spread function (c) Normalized raw and calibrated A-scan data on a logarithmic scale. 102

Figure 5.5 (a) Pig adipose tissue before applying nanoplates (b) Image of the tissue just after application of nanoplates droplet (c) Image of the tissue after the nanoplates are completely diffused (d) Variation of the pixel intensity along the marked (red) line. ....104

Figure 5.6 OCT images acquired at (a) 20 (b) 40 (c) 60, and (d) $80 \mathrm{~min}$ after the delivery of nanoplates (e) Average A-scan profiles. 105

Figure 5.7 Speckle variance OCT images demonstrating the distribution of SSNPs (a) without SSNPs, and (b)-(e) 20, 40, 60, and 80 min after silver nanoplate delivery 107

Figure 5.8 FDTD simulation results (a) Variation of LSPW with aspect ratio (b) Enhancement of the electric field due to the LSPR in GNR. (c) The scattering, absorption cross-section and total extinction plot.

Figure 5.9 (a) TEM image of GNRs (b) Extinction spectrum obtained via UV-Vis-NIR .110

Figure 5.10 OCT image of agar gel phantom embedded with $\mathrm{TiO}_{2}$ (a) Phantom without GNRs (b) Phantom with GNRs (c) Averaged A-scan data with and without GNRs.

Figure 6.1 Flow cell channel used for the biofilm growth and imaging using OCT .......................120

Figure 6.2 The positions of the sample and the reference reflectors.....

Figure 6.3 (a), (b) and (c) represent the cross-sectional images of an empty flow cell, flow cell with 1 week old biofilm and flow cell with 2 weeks old biofilm respectively ;(d), (e) and (f) represent the A-scan signal from an empty flow cell, flow cell with 1 week old biofilm and flow cell with 2 weeks old biofilm, respectively.

Figure 6.4 (a) Schematic of the planar flow cell configuration used for the biofilm growth and OCM imaging. (b) Enface image of the USAF chart demonstrating the lateral resolution of the imaging system. FS: scan lens; FF: tube lens 128

Figure 6.5 (a) Enface image of bottom surface of the flow cell channel (a) acquired by OCM (b) acquired by light microscopy. The image sizes are $1.3 \mathrm{~mm} \times 1.0 \mathrm{~mm}$ 130 
Figure 6.6 Enface images and edge detected images of the $K P-1$ microcolonies formed on the base of flow cell channel. Images (a), (c) and (e) correspond to biofilm growth on day 1, day 3, and on day 3; $2 \mathrm{~h}$ after perturbation. Images (b), (d) and (f) corresponding edge detected images. The image sizes are $1.150 \mathrm{~mm} \times 1.030 \mathrm{~mm}$.

Figure 6.7 Enface image of KP-1 biofilm on matured state (Day-3). (b), (c) and (d) shows the cross sectional images acquired from the vertical positions indicated by the red dotted lines and numbers respectively. 132

Figure 6.8 A-scan profiles (averaged) demonstrating a reduction of the reflection peak caused by the presence of the microbial.

Figure 6.9 (a) Standard Optical Density $\left(\mathrm{OD}_{600}\right)$ measurements and (b) Colony Forming Unit (CFU) measurements made by periodically sampling the flow-cell effluent. (c) Optical density measured via OCM and (d) planar density measurements of the biofilm via OCM .......135

Figure 7.1 Schematic of the proposed beam delivery system via spatial multiplexing 143

Figure A.1 Schematic of the swept source laser. 164

Figure C.1 OCT images of pig adipose tissue (a) Just after refrigeration (b) After three hours at normal temperature (c) GNRs droplet deposited over tissue sample just after refrigeration (d) 15 minutes after the GNR deposition. TS: tissue surface; CL: adiposecells. 170

Figure D.1 Selective photograph of the combined experiment, located inside an incubator. The OCM probe is seen located above the flow-cell coverslip, with a working distance of about $1 \mathrm{~cm}$. .171

Figure D.2 Photograph of imaging system including data acquisition and control .171

Figure E.1 Enface images of KP-1 biofilms at the bottom surface of the flow channel 


\section{LIST OF TABLES}

Table 2.1: Comparison of means square current, noise variance and SNR of three OCT

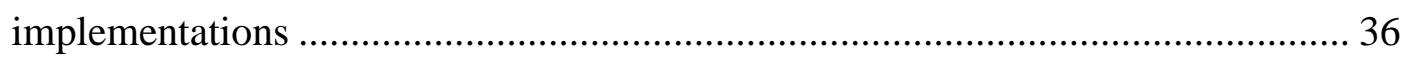

Table 5 .1: Optical extinction properties of different contrast agents ................................ 114 


\section{LIST OF SYMBOLS}

\section{$\underline{\text { Symbol }}$}

E

$e$

$\lambda$

$k$

$\omega$

$t$

$n$

$n_{g}$

$f$

D

$b$

C

$z$

$z_{f}$

S

$\overline{S(k)}$

$K\left(z-z_{f}\right)$

$\rho$

$R_{R}$

$R_{S}$

$R_{S, \min }$

\section{Definition}

Electric Field

Electronic Charge

Wavelength

Wavenumber

Angular frequency

Time

Refractive index

Group refractive index

Focal length

Diameter of the beam spot size

Confocal parameter

Concentration

Distance (Depth)

Position of the confocal gate

Spectral power

Spectral power density

Confocal point spread function

Responsivity of the photodetector

Power reflectivity of reference arm

Power reflectivity of sample arm

Minimum sample reflectivity 


\begin{tabular}{|c|c|}
\hline$R_{\text {Ref }}$ & Reflection peak before inoculation \\
\hline$R_{\text {new }}$ & Time varying reflection peak \\
\hline$Z_{R}$ & Distance to the reference mirror \\
\hline$Z_{S}$ & Distance to the sample surface \\
\hline$\Delta z$ & Path length difference \\
\hline$k^{\prime}$ & Initial wavenumber \\
\hline$k_{0}$ & Center wavenumber \\
\hline$\Delta k$ & $\begin{array}{l}\text { Bandwidth of the swept source in } \\
\text { terms of wavenumber }\end{array}$ \\
\hline$\Delta T$ & Sweep period \\
\hline$T$ & A-scan acquisition time \\
\hline$g(z)$ & Coherence gate function \\
\hline$\lambda_{0}$ & Central wavelength \\
\hline$\Delta \lambda$ & Bandwidth of the swept laser source \\
\hline$\Delta \mathrm{x}$ & Focal spot size \\
\hline$P_{S}$ & Incident signal power \\
\hline$P_{\min }$ & Minimum detectable power \\
\hline$I_{D}$ & Detector current \\
\hline$I_{D, \min }$ & Minimum detectable current \\
\hline$\sigma$ & Standard deviation of noise floor \\
\hline$M$ & Number of spectral channels \\
\hline$L$ & Depth of flow cell channel \\
\hline$L^{\prime}$ & Physical thickness of biofilm \\
\hline$B$ & Electrical bandwidth \\
\hline$B_{T D}$ & $\begin{array}{l}\text { Detection bandwidth of time- } \\
\text { domain system }\end{array}$ \\
\hline
\end{tabular}




\begin{tabular}{|c|c|}
\hline$B_{S D}$ & $\begin{array}{l}\text { Detection bandwidth of spectral- } \\
\text { domain system }\end{array}$ \\
\hline$B_{S D}$ & $\begin{array}{l}\text { Detection bandwidth of swept- } \\
\text { source system }\end{array}$ \\
\hline$\mu$ & Extinction coefficient \\
\hline$\mu_{b}$ & Back-scattering coefficient \\
\hline$\mu_{\text {Ref }}$ & $\begin{array}{l}\text { Extinction coefficient of reference } \\
\text { phantom }\end{array}$ \\
\hline$\mu_{R e f+G N R}$ & $\begin{array}{l}\text { Extinction coefficient of gold } \\
\text { nanorod embedded phantom }\end{array}$ \\
\hline$\circledast$ & Convolution \\
\hline$\Psi$ & Spectral resolution function \\
\hline$\delta_{r}$ & Instantaneous spectral width \\
\hline$\delta_{s} k$ & Spectral sampling interval \\
\hline$\sum$ & Sensitivity \\
\hline$\varphi$ & Spectral phase \\
\hline$\varphi(k, i)$ & Spectral phase function \\
\hline$\varphi_{\text {Disp }}$ & Dispersion induced phase \\
\hline$w(n)$ & Hamming window function \\
\hline$\Delta z_{\text {air }}$ & Axial resolution in air \\
\hline$\Delta z_{\text {eff }}$ & Effective axial resolution \\
\hline$Z_{\max }$ & Maximum imaging range \\
\hline$Z_{-d B}$ & Principal imaging range \\
\hline
\end{tabular}




\section{LIST OF ACRONYMS}

CA

CCD

CFU

$\mathrm{CM}$

CPI

CT

D

DAC

DAQ

ED

EPS

FDML

FDTD

FFPI

FFT

FTIR

FWHM

GNR

GPU

IFFT

IS

KP-1

LSPR
Cornea

Charged Coupled Device

Colony Formation Unit

Confocal Microscopy

Common Path Interferometer

Computed Tomography

Dermis

Digital to Analog Converter

Data Acquisition

Epidermis

Extracellular Polymer Substance

Fourier Domain Mode Locking

Finite Difference Time Domain

Fixed Fiber Fabry-Perot Interferometer

Fast Fourier Transform

Fourier-Transform Infrared Microscopy

Full Width at Half Maximum

Gold nanorod

Graphic Processing Unit

Inverse Fast Fourier Transform

Iris

Klebsiella Pneumonia

Localized Surface Plasmon Resonance 
LSPW

MI

MRI

MRT

MSD

MZI

NA

NB

NDFT

NIR

NMRI

NNC

NP

NU-FFT

OCM

OCT

OD

OFDI

OFDR

PA-1

PAS

PD

PTDI

PEG

PET
Longitudinal Surface Plasmon Wavelength

Michelson Interferometer

Magnetic Resonance Imaging

Magnetic Resonance Tomography

Mean Square Displacement

Mach Zehnder Interferometer

Numerical Aperture

Nail Bed

Non-Uniform Discrete Fourier Transform

Near Infra-Red

Nuclear Magnetic Resonance Imaging

Nearest Neighbor Check

Nail Plate

Non-Uniform Fast Fourier Transform

Optical Coherence Microscopy

Optical Coherence Tomography

Optical Density

Optical Frequency Domain Imaging

Optical Frequency Domain Reflectometry

Pseudonymous Aeruginosa

Photoacoustic Microscopy

Photodetector

Phase based Time Domain Interpolation

Polyethylene Glycol

Positron Emission Tomography 


\begin{tabular}{|c|c|}
\hline PIC & Photonic Integrated Circuit \\
\hline PLC & Planar Light Circuit \\
\hline PSF & Point Spread Function \\
\hline RIN & Relative Intensity Noise \\
\hline RPE & Retinal Pigment Epithelium \\
\hline $\mathrm{SC}$ & Sclera \\
\hline SCLM & Scanning Confocal Laser Microscopy \\
\hline SDOCT & Spectral Domain Optical Coherence Tomography \\
\hline SDS & Sodium Dodecyl Sulfate \\
\hline SNR & Signal-to-Noise-Ratio \\
\hline SOCT & Spectroscopic Optical Coherence Tomography \\
\hline SPR & Surface Plasmon Resonance \\
\hline SSNP & Silica-coated silver nanoplates \\
\hline SSOCT & Swept Source Optical Coherence Tomography \\
\hline STXM & Scanning Transmission X-Ray Microscopy \\
\hline SV & Speckle Variance \\
\hline TDOCT & Time domain Optical Coherence Tomography \\
\hline TEM & Transmission Electron Microscopy \\
\hline TFSF & Total Field Scattered Field \\
\hline $\mathrm{TM}$ & Trabecular Mesh \\
\hline UHR & Ultra-High Resolution \\
\hline USAF & United States Air Force \\
\hline VCSEL & Vertical Cavity Surface Emitting Laser \\
\hline
\end{tabular}




\section{Chapter 1: Introduction}

This chapter begins with the background and motivation for undertaking this thesis, followed by a brief review on the optical coherence tomography and its application in biomedical area. A short note on the importance and current research challenges with the optical coherence tomography are explained. The main objectives and scopes of this thesis will be discussed, followed by a block diagram representation of the research roadmap planned for meeting the desired objectives of the thesis. The chapter concludes with the organization of the whole thesis.

\subsection{Background}

Biomedical research mainly focuses on detection, diagnosis, prevention and treatment of diseases which ultimately leads to better health. Ultra-sensitive detection and imaging methods are enabling current technologies to evolve into a new generation of medical diagnosis techniques. Advancement in bio-imaging technologies provides excellent capabilities for the healthcare and disease management which includes early detection of diseases, disease prevention, injury assessment, guided surgery and interventions, and noninvasive monitoring of treatment effects. Furthermore, these technologies are capable of probing complex and dynamic biological processes thereby offering tremendous capabilities to accelerate basic research and clinical diagnostics.

Optical coherence tomography (OCT) has evolved to become a powerful imaging modality in biomedical research and clinical medicine. OCT performs high resolution crosssectional and three-dimensional (3D) volumetric imaging of internal microstructures of the 
biological specimen in-situ and in real-time [1-3]. Unlike computed tomography (CT) and positron emission tomography (PET), OCT makes use of non-ionization radiation, specifically, near infrared (NIR) radiation for imaging. Imaging with NIR window (800 nm-1300 nm) offers better penetration depth $(1-3 \mathrm{~mm})$ in biological samples. OCT provides high spatial resolution $(1-15 \mu \mathrm{m})$ close to the histopathology and allows optical biopsy without the need for tissue excision or processing of specimens. This resolution is one or two order finer than ultrasound imaging. Highly flexible instrumentation designs based on the fiber optic technology allows OCT to integrate with a wide range of clinical instruments such as surgical microscopes, endoscopes, catheters, and imaging needles. These unique features have made OCT a powerful imaging modality with applications spanning many clinical specialties, fundamental scientific, developmental biological research, as well as non-biological applications.

OCT is a low coherence interferometric technique that constructs tomographic images using depth dependent back reflected NIR signals from the tissues. Low coherence interferometry enables accurate measurement of optical echoes from the closely packed layers and provides an excellent optical sectioning capability. Earlier version of this technique is known as the time-domain OCT (TDOCT), which acquires the depth resolved images by scanning the reference arm of the interferometer [1, 4]. Mechanical movement of the reference mirror limits the imaging speed (A-scan rate) of TDOCT to only a few kHz. Alternatively, Fourier-domain configurations were introduced for high speed OCT imaging. Fourier-domain OCT (FDOCT) systems avoid the translatory motion of the reference mirror and facilitate image construction via spectrally resolved detection of the interferogram [5, 6]. Depending on the methodology adopted for spectral discrimination, 
FDOCT systems are categorized into spectral-domain OCT (SDOCT) and swept-source OCT (SSOCT) systems. In SDOCT systems, the interferometer is illuminated by a broadband source and the spectral components are spatially resolved and detected by a combination of grating and line scan camera [5, 7]. In the case of SSOCT, a rapidly sweeping narrow bandwidth laser source is used in the system and the spectral fringes are coherently detected by a photodetector [8-10]. Recent trends in OCT imaging are more focused on FDOCT systems because of the inherent capability to perform high speed imaging with improved sensitivity $[11,12]$. Among the FDOCT systems, SSOCT systems have gained considerable attention due to the potential for high speed imaging and low cost-detection at longer wavelengths. Furthermore, recent developments in the sweptsource technology and the availability of sophisticated data acquisition systems enable imaging with longer depth range and reduced sensitivity roll-off [13-15]. This has made SSOCT a better choice for many diagnostic imaging applications and biomedical research.

\subsection{Motivation}

The current research trends in OCT technology are more focused on enhancing its imaging capability by addressing various fundamental research challenges in optics and electronics. Similarly, intense efforts are directed towards bringing innovations in the architectural design and instrumentation schemes of OCT to extend its applicability for many complex imaging scenarios. Most of the OCT systems adopt fiber-optic configurations due to their significant advantages in terms of signal quality, compactness, stability and easiness of the light distribution into the sample. The heart of every OCT system is an interferometer. The most common interferometer used in the development of OCT configuration is the fiber-optic Michelson interferometer (MI). Despite of its regular 
implementation, the standard MI configuration is not a compact and efficient design for many in-vivo imaging applications. For example, the reference arm of MI is generally bulky and alignment sensitive due to the presence of optical elements such as mirror, neutral density filter and dispersion compensation elements. This affects the compactness, portability and stability of the OCT systems, and restricts their applicability in many in-situ imaging applications. Although there is a great progress in the development of MI based technologies, there remains significant interest in developing efficient fiber-optic interferometer configuration that are highly compatible for in-vivo and/or in-situ applications.

Imaging with high resolution and sensitivity is highly desirable for diagnostic applications. High resolution OCT systems can image cellular and extra cellular structures in-vivo quantitatively and non-invasively and thus can improve the diagnostic accuracy by eliminating the sampling error and artifacts. However, the axial resolution and sensitivity of OCT systems are often limited by the non-linearity associated with the spectral sampling techniques used in the system design as well as the system and media dispersion. The mechanical wavelength tuning adopted in the swept-laser sources causes the wavenumber to be swept nonlinearly and to vary within subsequent sweeps [16-18]. This results in the acquisition (sampling) of OCT signals unevenly in $k$-space. The Fourier transformation of unevenly sampled fringes leads to significant reconstruction errors and drastically compromises the performance in terms of axial resolution and sensitivity [19, 20]. This necessitates the resampling of OCT signals in uniform $k$-space intervals prior to the image reconstruction. Various hardware and software methods have been proposed to address this issue. An external $k$-clock based hardware triggering has been demonstrated 
for uniform sampling in OCT $[21,22]$. However, inaccurate triggering caused by the electronic jitter creates problems in this approach. Alternatively, reference signals generated using auxiliary interferometers have been used to calibrate interferograms via post-processing methods [23-25]. The accuracy of these approaches is limited due to the functional approximations used in their algorithm. Most of these calibration schemes demand additional hardware resources, leading to an increased system complexity and reduced cost efficiency. On the other hand, stand-alone schemes based on the numerical algorithms have been widely used for calibration [26, 27]. However, real-time calibration with these schemes require high performance computational platform such as graphics processing unit (GPU) due to their computational complexity and memory requirement. This shows that the performance of the existing calibration scheme is limited in terms of accuracy or computational efficiency. Moreover, most of these approaches are expensive and not feasible for real-time calibration in high speed SSOCT systems.

Another potential factor that limits the axial resolution and sensitivity is the dispersion mismatch between the sample and reference arm of the interferometer. Dispersion is mainly caused by dependence of the refractive index on wavelength. For the fiber-based OCT setup, the dispersion is inevitable because broad band light propagates in optically dense materials such as glass, tissue and water. Dispersion is often corrected physically by matching the media in the sample and reference arms, or by placing compensating optics such as variable thickness prism in the optical path of one of the arms [28, 29]. However, compensation using physical optics method is least preferred due to the space and access constraints. Alternatively, numerical methods are preferred for dispersion correction since they correct dispersion automatically without increasing hardware complexity [30-32]. 
Another important aspect of OCT imaging is image contrast, which has a significant impact on its diagnostic capabilities and clinical utility. The contrast of conventional OCT is limited when the pathological tissue is optically or morphologically identical to normal tissue. This limits the diagnostic capability of OCT at the earlier stages of the diseases. The contrast of OCT images can be enhanced by the administration of passive or targeted exogenous contrast agents at the specific site of interest. Contrast agents applied to the specific sites of interest clearly delineate selected tissues from their surroundings. In addition to targeted imaging of cells or tissues, contrast agents can also serve as multifunctional agents for drug delivery and photothermal therapy. Metallic nanoparticles are widely used as contrast agents in optical imaging owing to their localized surface plasmon resonance (LSPR) induced scattering and absorption properties, and spectral tunability [33, 34]. Gold nanoparticles are extensively used for contrast enhancement and photothermal applications because of their excellent biocompatibility and nontoxicity [ 35 , 36]. However, most of these studies were focused on nanostructures, whose LSPR spectral peaks fall below $1000 \mathrm{~nm}$. Recently, imaging with longer wavelength window such as $1300 \mathrm{~nm}$ is highly preferred for diagnostic applications due to the increased penetration depths and reduced background scattering $[37,38]$. However, very few nanostructures are reported in this wavelength range for contrast enhancement in OCT imaging and photothermal applications. Therefore, the development of plasmonic nanoprobes whose LSPR peak falls in the $1300 \mathrm{~nm}$ window are still under active research.

In order to investigate the applicability and effectiveness of plasmonic nanoprobes for contrast enhancement and therapy, their optical extinction properties must be precisely quantified. The real-time monitoring of nanoparticle distribution and their transport in 
biological tissues are required to be performed in order to study their effective coverage over lesion area and tissue clearance. LSPR characterization using conventional methods such as integrated sphere and spectroscopy are sensitive to many experimental parameters and show large fluctuations [39-41]. Moreover, they are not feasible for the in-vivo characterization of nanoparticles embedded in biological tissues. Similarly, existing methodologies used for the investigation of nanoparticles penetration, such as mean square displacement and Brownian diffusion models are inefficient for assessing nanoparticle distribution over large area $[42,43]$. On the other hand, spatial and temporal variation of the OCT signal can provide real-time information of optical performance and transportation of the nanoprobes over large volume. Accordingly, quantitative assessments of OCT raw data and images via intelligent signal processing algorithms have turn to be a future exploratory area in nanomedicine.

Imaging at cellular and subcellular resolutions with optical imaging techniques is important in developmental biology. Over the past few years, there is an increased interest in exploring the potential of high resolution optical imaging and advanced metrological techniques for the real-time in-situ investigations of microorganisms such as biofilms. Various optical and non-optical schemes have been reported for the investigation of biofilms [44-47]. The non-optical schemes are generally bulky and not feasible for in-situ and online monitoring of the biofilms. Among the optical imaging schemes, confocal microscopy $(\mathrm{CM})$ is widely accepted since it allows high resolution enface and 3D imaging of biofilms. However, this approach works only with the staining of the cells or bacteria which limits its applicability for the in-vivo investigation of natural biofilms. In addition, $\mathrm{CM}$ offers a restricted penetration depth and requires the movement of the focal plane for 
z-slicing and 3D image acquisition. This prevents them to use for online monitoring of biofilms. On the other hand, the capability of OCT to perform label free imaging with improved speed and penetration has made it as a promising tool for in-vivo and/or in-situ monitoring of biofilms [48, 49]. Moreover, the metrological capabilities of OCT allow precise determination of various physical and optical parameters as well as the real-time quantification of biofilms. However, only limited studies were carried out in this direction and still remains as a potential area of research.

This thesis focuses on the research and investigation into a compact swept source-based optical coherence system with enhanced imaging and metrological capabilities. A fiberoptic interferometer with non-reflective reference arm is introduced as an alternative to conventional MI for a compact and stable design. Imaging with enhanced axial resolution and sensitivity is accomplished by implementing an accurate spectral phase-based automatic calibration scheme and a dispersion correction scheme. The proposed methodologies for wavenumber calibration and dispersion correction do not require additional equipment or physical optics, which further simplifies the OCT system. The plasmonic silver and gold nanoprobes are introduced for contrast enhancement in OCT imaging around $1300 \mathrm{~nm}$. A numerical approach based on OCT is proposed for the precise quantification of LSPR induced extinction cross-section of nanoparticles. The specklevariance OCT imaging is demonstrated as an efficient method for investigating the nanoparticle distribution and their transport in biological tissues. The potential of optical coherence techniques for in-situ and quantitative investigation of the biofilms is explored in this thesis. The metrological capabilities of OCT for real-time assessment of optical and 
physical parameters of the biofilms are also demonstrated. In this context, this research has the following objectives and scopes.

\subsection{Objective}

Based on the previous sections, it can be inferred that the realization of an optical coherence imaging system with improved performance in terms of accuracy and contrast, is one of the research thrust area. Moreover, the potential of such an imaging system for real-time quantitative imaging of biological specimens is an important and on-going research in clinical medicine and developmental biology. The fundamental focus of this thesis hence aims at exploring the research and development of a swept source-based optical coherence imaging system with enhanced imaging capabilities in terms of axial resolution, sensitivity and contrast. The focus of this thesis is further extended to novel qualitative and quantitative approaches for the in-situ and online monitoring of microbial communities using optical coherence tomography.

The major objectives of this research in this context can be summarized as:

Research into the enhancements of imaging capabilities of optical coherence imaging systems in terms of novel instrumentation concepts and contrast enhancement methodologies.

Research into the metrological capabilities of optical coherence tomography/microscopy for real-time quantitative and in-situ investigations.

The following section outlines the scope of the research work carried out to meet these desired objectives. 


\subsection{Scope}

The major areas of this research are outlined as follows:

Configuration and experimental characterization of the non-reflective reference arm based SSOCT system and the performance analysis.

Conceptualization, configuration and experimental characterization of a novel automatic calibration scheme for SSOCT system and its performance analysis.

Implementation of a novel spectral phase-based automatic calibration scheme for SSOCT system, followed by its performance analysis and comparison.

Implementation of a spectral phase-based dispersion correction scheme followed by its performance analysis.

Theoretical and experimental investigation into contrast enhancement in OCT using novel nanoprobes.

Implementation of a novel approach for characterization of localized surface plasmonresonance in metallic nanoparticles using OCT and comparison with other standard techniques.

Experimental investigation into the nanoparticle transport and distribution in biological tissues using speckle variance OCT imaging.

Configuration and experimental characterization of a swept source-based optical coherence microscopy for the investigation of bacterial biofilms.

Theoretical and experimental investigation into the real-time and in-situ assessment of optical and physical parameters of bacterial biofilms. 
Experimental investigation into the real-time imaging of the development of thick and planar biofilms.

Theoretical and experimental investigation into the real-time quantitative assessment of growth dynamics of the biofilm using optical density and planar density.

A detailed research road map outlining the research methodology for this thesis is given in the flow chart (See Figure 1.1).

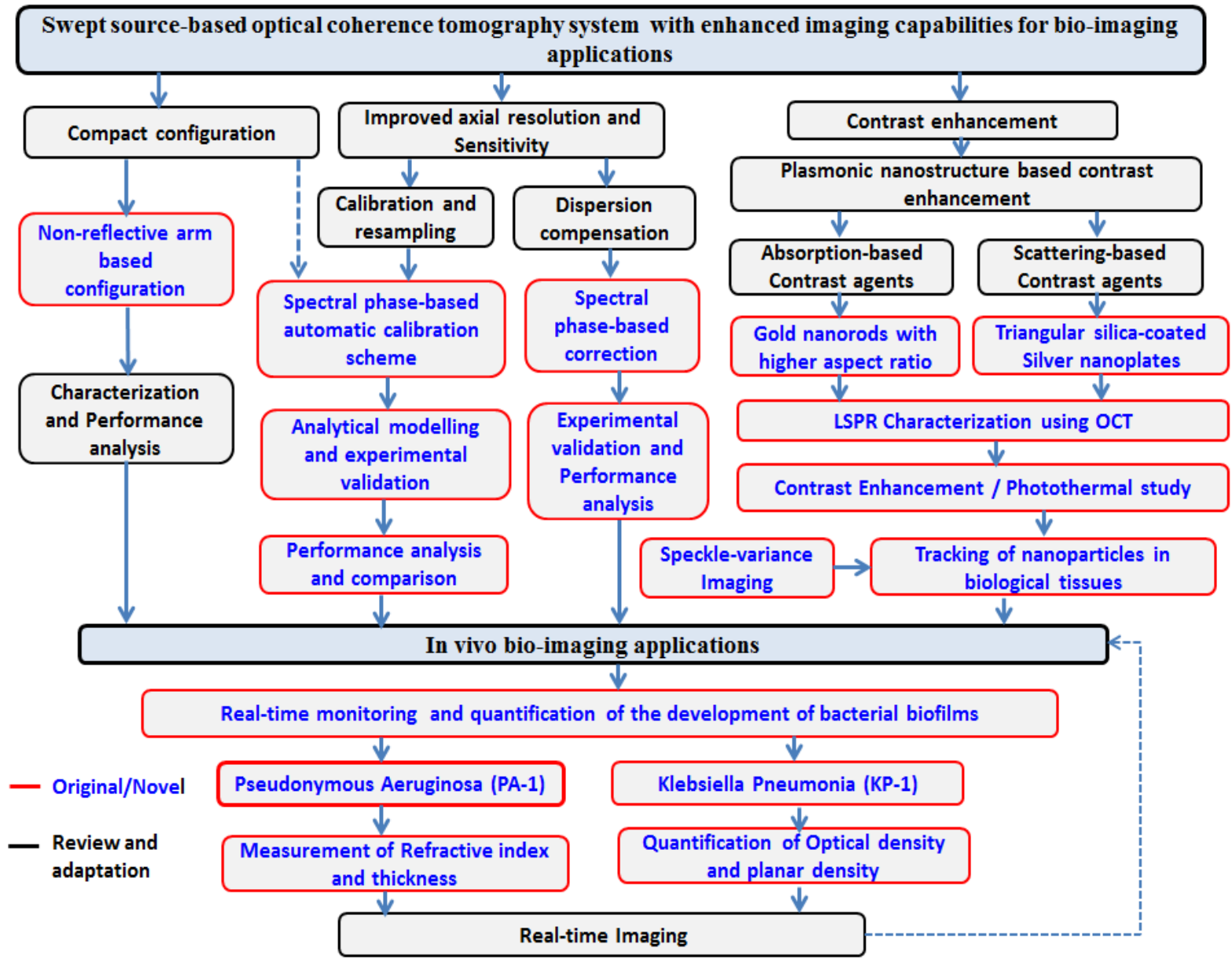

Figure 1.1 Block diagram representation of the whole research methodology implemented for the research thesis. 


\subsection{Organization of the thesis}

This thesis is divided into seven chapters. Each chapter starts with a short note on the main contents of that chapter.

Chapter 1 is an introduction chapter which gives a brief outline on the current status of the research problem considered. The motivation, scopes and objectives of the thesis are also discussed. A summary regarding the limitations of OCT imaging are briefly discussed by highlighting the need for new methodologies to overcome the current limitations. A block diagram representation of the proposed thesis project explaining the methodologies is also presented. The organization of the whole thesis is given in the last section of this chapter.

Chapter 2 presents the state-of-art-of OCTs and their working principles. The chapter starts with the historical background of OCT and its developments over time. Detailed description of different imaging parameters such as spatial resolution, sensitivity, and imaging depth are given along with the theoretical background. Various design considerations and research challenges of OCT imaging are discussed. The different methods reported for addressing the research challenges are explained and compared. The outcome of the literature review is discussed briefly at the end of this chapter.

Chapter 3 describes the design and development of a compact SSOCT system. Fundamental theoretical concepts and various design considerations of SSOCT system are presented at the beginning of this chapter. A comprehensive analysis on the instrumentation scheme including hardware and software configurations is systematically illustrated by highlighting the technical relevance. Experimental and theoretical characterizations of the developed system are presented. The performance of the proposed SSOCT architecture is analyzed based on major imaging parameters such as axial 
resolution, sensitivity and imaging depth. The imaging results obtained from different biological samples are presented at the end of this chapter.

Chapter 4 describes the real-time automatic calibration scheme implemented in the proposed SSOCT system. This chapter begins with explaining the proposed methodology for the generation of reference signal using an automatic method. This is followed by an explanation of a novel spectral-phase approach for real-time calibration of OCT signal. Illustration of the proposed real-time automatic calibration using a mirror and a biological sample are presented. The performance of the proposed calibration scheme is evaluated based on major imaging parameters such as axial resolution, sensitivity roll-off, imaging speed and quality of the acquired images. A comparison with existing schemes is also presented.

Chapter 5 illustrates the numerical investigation and experimental study conducted for contrast enhancement in OCT using exogenous contrast agents. This chapter starts with the discussion of LSPR-induced optical properties of silver and gold nanoparticles. Contrast enhancement using silica-coated silver nanoplates (SSNPs) and gold nanorods (GNRs) are presented in two different sections. Quantification of extinction cross-sections using novel approaches based on OCT are presented for both nanoparticles. The characterization of optical properties using spectroscopy and finite difference time-domain (FDTD) are also illustrated. The investigation of contrast enhancement and diffusion characteristics of SSNPs in biological tissue is presented. The absorption based contrasting properties of the GNRs are described towards the end of this chapter.

Chapter 6 illustrates the real-time quantitative investigation of bacterial biofilms using OCT and OCM techniques. The potential of OCT for high resolution cross-sectional imaging of Pseudonymous Aeruginosa (PA-1) is illustrated at the beginning of the chapter. 
This is followed by an illustration of real-time quantification of optical and physical parameters of PA-1 biofilm using OCT. In the following section, real-time in-situ monitoring of planar biofilms of Klebsiella Pneumonia (KP-1) using optical coherence microscopy is demonstrated. The enface images are presented for an efficient visualization of the earlier development of KP-1 biofilms and their transient response under perturbation. The proposed methodologies for real-time measurement of optical density and planar density of biofilms are demonstrated at the end of this chapter.

Chapter 7 is the last chapter of the thesis. It gives the conclusion for the thesis along with the guidelines for future works. It explains the important original research contributions made as part of the submitted thesis. All the theoretical and experimental works associated with the proposed research for fulfilling the required objectives are concluded here. Also, suggestions are provided for extending the applications areas of the proposed system by incorporating additional elements.

The next chapter overviews the state-of-the-art literature survey relevant to the proposed research topic to identify the potential research gap and required improvement. 


\section{Chapter 2: Literature Review}

This chapter reviews the earlier work done in the related areas that are relevant to the proposed thesis. This chapter begins with the introduction to optical coherence tomography, including the working principle and different implementations which are explained and compared. The following part of the section explains different characteristic OCT parameters, such as sensitivity, signal to noise ratio and optical resolution. Various research challenges in optical coherence imaging related to resolution and contrast are presented based on the reported works. Different approaches for solving these issues are discussed and compared. The outcome of literature review is discussed briefly towards the end of this chapter

\subsection{Introduction}

Until the last century, clinical medicine was dependent on the clinical tools such as thermometer, microscope, stethoscope and knife for diagnostics and therapeutic purposes. In bioresearch, structural studies on the organisms could be only performed at the organ level by anatomically studying the body. Functional and molecular level interactions inside the organisms were a myth and even structural information at tissue and cellular level was a difficult task to achieve. Until 1896, there was no means to study or evaluate the hidden internal world of the human body. The discovery of X-ray marked a revolution in the field of medical science and new branch of science called medical imaging was realized. Today, imaging technology is arguably one of the fastest growing and heavily contributing areas within the biomedical and developmental biology research. 
Today, there are several imaging methodologies available for the biomedical and developmental biology research. Noninvasive radiation technologies such as computer tomography (CT), magnetic resonance tomography (MRT) and positron emission tomography (PET) are widely used in the clinical medicine and other areas of biomedical research. Although these methodologies are capable of imaging large volumes in human body, they are complex, expensive, and demand more space for their clinical applications. Imaging techniques such as $\mathrm{CT}$ and PET are based on ionization radiation, which restricts their applicability in human imaging. Performance of the conventional clinical systems is further limited in terms of spatial resolution. Typical resolutions of conventional imaging systems ranges from $\sim 0.5 \mathrm{~mm}$ to $\sim 1 \mathrm{~mm}(\mathrm{CT}$ and MRT), and several millimeters (PET), which is insufficient for many medical applications [50, 51]. Alternative, less complex approaches based on ultrasound and optical techniques offer high resolution images using non-ionization radiation [52].

A typical ultrasound system has a spatial resolution of $100 \mu \mathrm{m}$ and an imaging depth of several centimeters. However, there exists a trade-off between axial resolution and imaging depth depending on the ultrasound frequency used. A high frequency ultrasound system can provide a resolution up to $\sim 15 \mu \mathrm{m}$, by compromising the imaging depth to only a few millimeters [53]. On the other hand, a high resolution imaging at submicron level can be accomplished via optical methods such as confocal microscopy. In confocal microscopy, the axial and lateral resolution is linked by the beam diffraction and the imaging depth is limited to only a few hundred microns. Optical coherence tomography (OCT) is another optical imaging modality that offers higher axial resolution compared to ultrasound imaging, and better penetration than confocal microscopy. Thus, OCT fills the gap 
between confocal microscopy and other standard clinical imaging modalities in terms of imaging depth and resolution, as illustrated in Figure 2.1. Unlike confocal systems, the axial and lateral resolutions of OCT are decoupled. This enables OCT to use low numerical aperture (NA) focusing, which increases the imaging field. A typical OCT system can offer an axial resolution of $\sim 10 \mu \mathrm{m}$ and a penetration depth of $\sim 1-3 \mathrm{~mm}$ in biological tissues [54]. Ultra-high resolution OCT systems are also introduced to provide a resolution up to $1-2 \mu \mathrm{m}$ [55]. In spite of the small imaging volume and shallow penetration depth, OCT is preferred to CT and ultrasound imaging in many medical imaging applications because of its potential high resolution imaging with fast image acquisition rate.

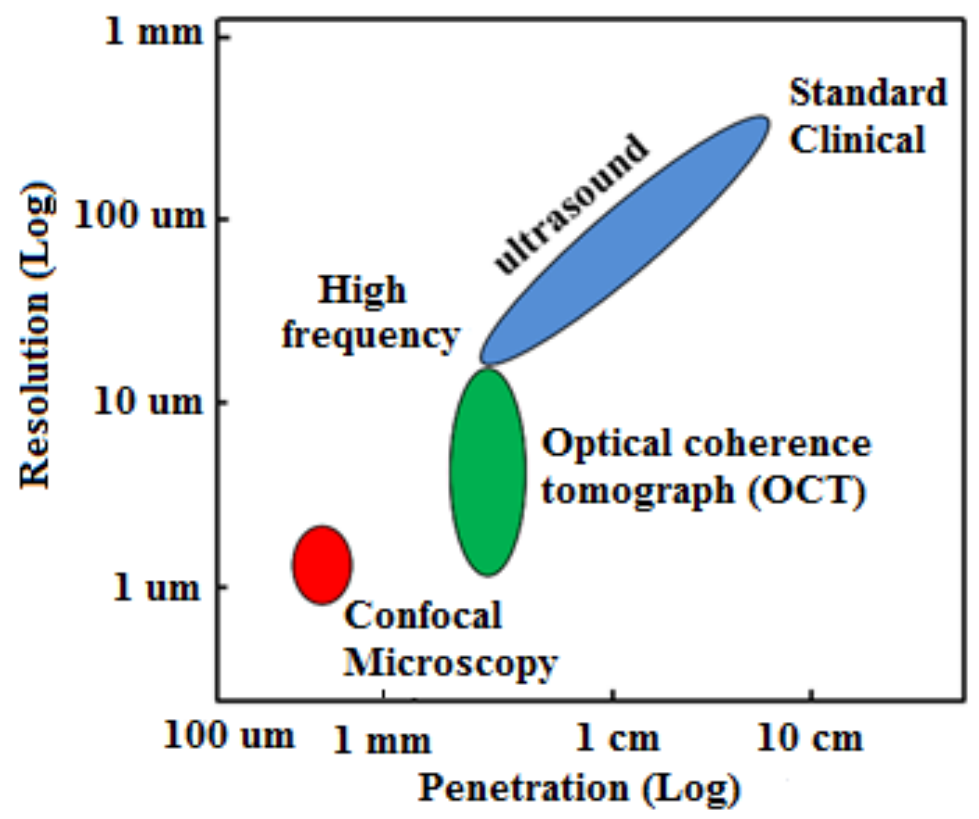

Figure 2.1 Performance of different biomedical imaging techniques with respect to image penetration and achievable resolution (Logarithmic scale) [54]. 


\subsection{Historical Background of OCT}

OCT construct cross-sectional images similar to the ultrasound imaging, except that it makes use of optical waves instead of low frequency sound waves. In medical ultrasonography, conventional electronics schemes are used to detect the magnitude and time delay of back-reflected acoustic waves from the tissue structures. Due to the ultrafast nature of light, the time delay between the optical reflections will be too short to be resolved by the electronics detectors. This prompted the researchers to seek alternate ways to measure optical echoes from the tissue layers. Optical ranging in biological systems was firstly demonstrated in 1971, where an ultra-fast electro-optic shutter was used to separate short light pulses back-scattered from tissue structures [56]. Based on this approach, the cataract microstructure in rabbit eyes has been performed. However, the resolution achievable in this method was poor (> 1mm). In another approach, short pulses backscattered from different tissue layers were resolved by non-linearly mixing with a delayed reference pulse using a cross-correlation setup [57]. This approach significantly improved the axial resolution, and an axial resolution of $15 \mu \mathrm{m}$ in air was obtained. However, a reduced sensitivity of $\sim 70 \mathrm{~dB}$ was obtained in this case. The sensitivity is defined as the ratio of the incident signal power on the sample to the minimum detectable power. A minimum sensitivity of $\sim 90 \mathrm{~dB}$ is required for obtaining good quality images of thick biological samples [56]. Finally, the breakthrough came with the technique of low coherence technique, which enabled the measurement of optical echoes from closely packed layers with high resolution and sensitivity. This technique namely, optical coherence-domain reflectometry was widely used for the optical evaluation of reflection sites in waveguides and the miniaturized optical fibers [58, 59]. The first biological application with this technique was realized in 1988 by measuring axial length of the 
human eye in-vivo [60]. The applicability of this technique for tomographic imaging was firstly demonstrated by Huang et al., in 1991 [1]. In this work, cross-sectional images of human coronary artery and retina ex-vivo were presented. The axial resolution and sensitivity of the first OCT system were $15 \mu \mathrm{m}$ and $93 \mathrm{~dB}$, respectively.

\subsection{Operating wavelength ranges for OCT}

The wavelength window chosen for OCT imaging has a significant impact on the image quality, contrast and the achievable penetration depth in biological tissues. Optical power in biological tissue is mainly attenuated by the wavelength dependent optical scattering and absorption. Therefore, the wavelength window chosen for OCT imaging is generally determined based on the wavelength dependent absorption/scattering characteristics of the sample. The availability of optical sources and detectors also plays a crucial role in determining the wavelength for imaging. The optical absorption in tissue is mainly contributed by the melanin, hemoglobin and water content present in the tissues [61]. Accounting to the reduced water absorption and simple accessibility of light sources and detectors, retinal imaging was firstly performed at $800 \mathrm{~nm}$ [1]. This wavelength is still a dominant wavelength regime in clinical systems for the retinal imaging. Alternatively, $1060 \mathrm{~nm}$ is introduced for retinal imaging due to the reduced water absorption and lower dispersion. A comparative study shows that imaging at $1060 \mathrm{~nm}$ offers high quality images with improved penetration, capable of resolving retinal structures below retinal pigment epithelium (RPE) [62].

Besides optical absorption, scattering also determines the tissue penetration and image quality. Most of the OCT applications except retinal imaging, demand imaging of highly scattering tissues, where the signal loss is mainly contributed by the optical scattering. The 
wavelength dependent scattering in most of the tissues can be expressed as the power relation $a \lambda^{-b}$, where $b$ typically ranges from 1 to 2 [63]. Therefore, imaging at longer wavelength offers reduced scattering loss; thereby an improved penetration at same OCT sensitivity can be achieved. Imaging at $1300 \mathrm{~nm}$ spectral band is accepted as a powerful imaging window for OCT imaging due to reduced scattering loss and the availability of the suitable sources and detectors. The improved imaging penetration at $1300 \mathrm{~nm}$ relative to $800 \mathrm{~nm}$ spectral band is experimentally demonstrated by imaging human epiglottis tissue ex-vivo [64-66]. Besides the normal tissue imaging, $1300 \mathrm{~nm}$ OCT is widely used for the imaging of the anterior chamber of eye [18].

\subsection{Principle of OCT}

The low coherence interferometry is the driving principle of OCT. Figure 2.2 shows the schematic of an OCT imaging setup using a simple Michelson interferometer. Let $E_{i}=$ $E(k, t) e^{j(k z-\omega t)}$ represents electric field of the spectrally broadened light waves used to illuminate the interferometer. Where $k$ and $\omega$ represents the wavenumber and angular frequency, respectively.

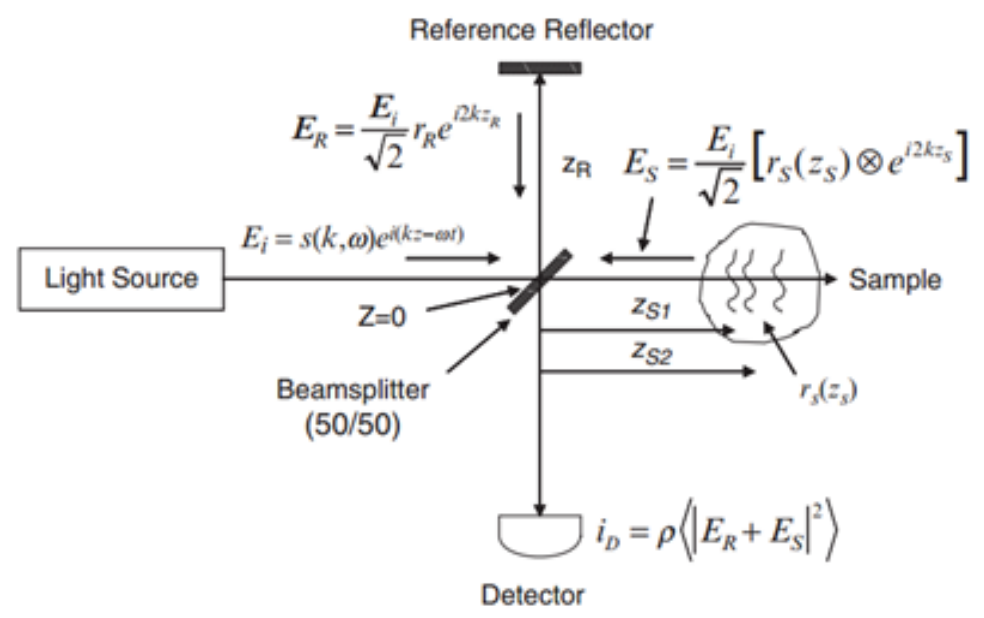

Figure 2.2 Schematic of Michelson Interferometer used in OCT [54] 
The beam splitter divides the source intensity into two halves. The beam that transmits through the beam splitter will have the form $E_{s}=\frac{E(k, t)}{\sqrt{2}} e^{j(k z-\omega t)}$ and the reflected beam will obtain a phase shift $\pi$ and takes the form $E_{R}=\frac{E(k, t)}{\sqrt{2}} e^{j(k z-\omega t+\pi)}=-\frac{E(k, t)}{\sqrt{2}} e^{j(k z-\omega t)}$. The light waves reflected from the sample and reference mirror have a phase difference depends on the path length difference between the interferometer arms. The superimposed electric field will therefore be $E_{T}=E_{R}+E_{S}$. The interference signal detected by a square law detector follows the relation $[1,54]$

$$
I_{D}=\rho\left\langle E_{T} E_{T}^{*}\right\rangle
$$

Where $\rho$ represents the responsivity of the detector and $E_{T}^{*}$ is the complex conjugate of the electric field. This gives a real signal

$$
I_{D}(k)=\frac{\rho}{4} S(k)\left[R_{R}+R_{S} \pm 2 \sqrt{R_{R}} \sqrt{R_{S}} \cos (2 k \Delta z)\right]
$$

Where $S(k)=\left\langle E^{2}(k, t)\right\rangle$ represents the spectral power of the light source, and $\Delta z=Z_{R}-$ $Z_{S}$ is the optical path length difference between the interferometer arms. $R_{R}$ and $R_{S}$ represents the power reflectivity of each reflector.

Equation (2.2) shows that detector current has three terms. The first two terms represent the DC, which is the product of spectrum power and the sum of the reflectivities of reference and sample arm. The third term represents the cross-correlation component, which contributes to the OCT signal. The third term further shows that, OCT signal power is proportional to $\sqrt{R_{R} R_{S}}$, and that can be controlled via reference arm reflectivity. This heterodyne gain allows OCT to achieve higher sensitivity greater than $100 \mathrm{~dB}$. 
In the case of $\mathrm{N}$ reflectors with individual power reflectivity $R_{s 1}, R_{s 2}, \ldots R_{S N}$, the extension of the analysis gives [54]

$$
\begin{aligned}
& I_{D}(k)=\frac{\rho}{4}\left[S(k)\left[R_{R}+R_{S 1}+R_{S 2} \ldots\right]\right]+ \frac{\rho}{4}\left[S(k) \sum_{n=1}^{N} \sqrt{R_{R} R_{S n}}\left(\cos 2 k\left(Z_{R}-Z_{s n}\right)\right)+\right. \\
& \frac{\rho}{4}\left[S(k) \sum_{n \neq m=1}^{N} \sqrt{R_{S n} R_{S m}}\left(\cos 2 k\left(Z_{s n}-Z_{s m}\right)\right)\right.
\end{aligned}
$$

The first two terms in the Equation (2.3) represent the DC term and cross-correlation term, respectively. The third term represents the autocorrelation signal, caused by the mutual interference between the signals within the sample. Figures 2.3 (a) and (b) demonstrate the spectral interferogram corresponding to single reflector and multiple reflectors, respectively. Figure 2.3(a) represents a single sinusoidal signal with a dc offset. It is worth to mention that, the period of the cosinusoid is proportional to the path length difference between the sample and reference reflectors. In the case of multiple reflectors, interferogram modulated by multiple sinusoidal signals were obtained as shown in Figure 2.3 (b).
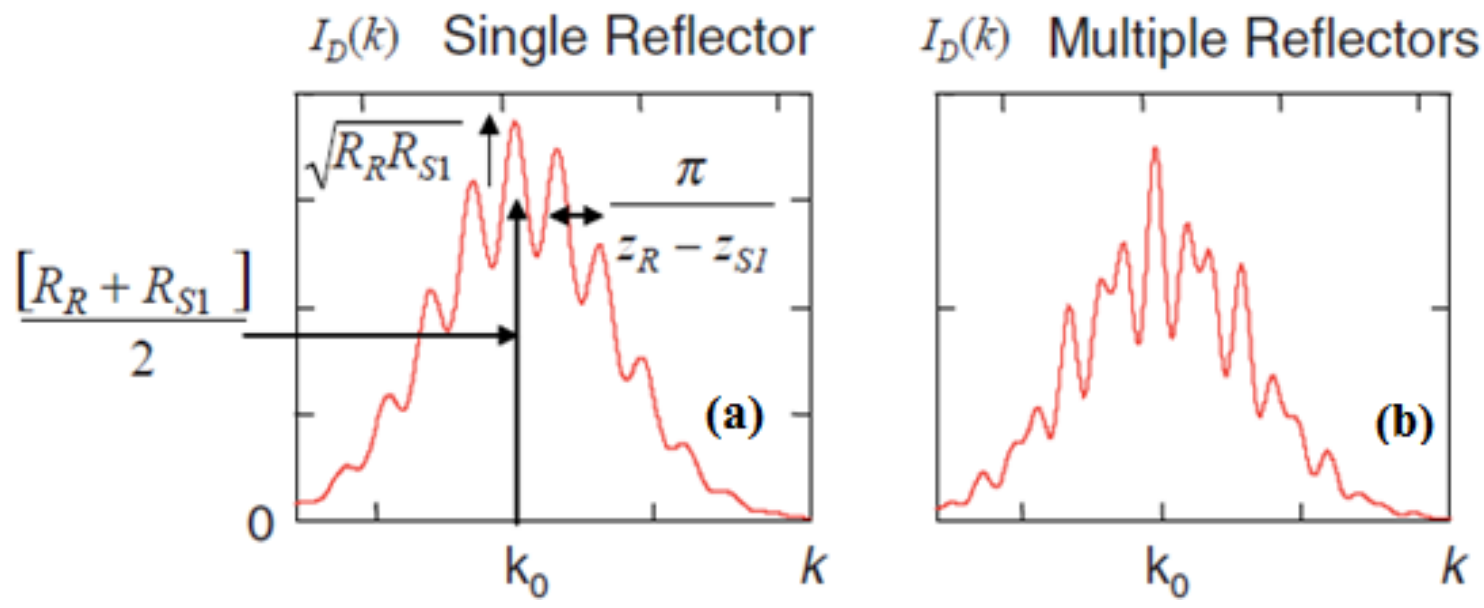

Figure 2.3 Interferogram obtained for (a) Single reflector (b) Multiple reflectors [54] 


\subsection{Different variants of OCT systems}

The OCT configurations are majorly classified into Time-domain OCT (TDOCT) systems and Fourier-domain OCT (FDOCT) systems based on the procedure by which the depth reflectivity profile, or the A-scan is acquired.

\subsubsection{Time-domain OCT (TDOCT)}

The time-domain configuration is the earlier version of the OCT system, which is solely based on low coherence interferometry. The typical schematic of the TDOCT is shown in Figure 2.4. The imaging system is illuminated by a spectrally broadened source and the interferogram is recorded via a single photodetector. In TDOCT, a coherence gate formed by the short coherence length, allows the selective interrogation of the back scattering from a certain depth in the sample. Thus, the depth information is recorded by shifting the coherence gate across the sample depth by moving the reference mirror.

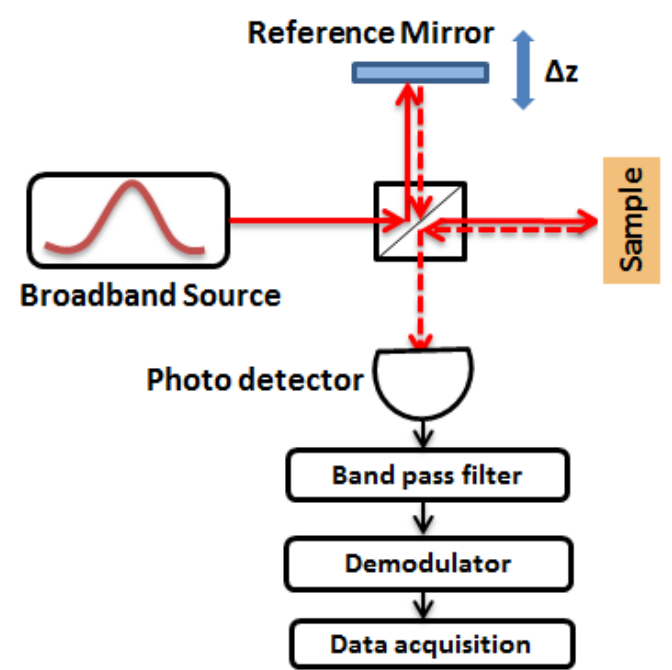

Figure 2.4 Schematic of Time-domain OCT system [50]

In TDOCT, the photodetector receives all the spectral components simultaneously. Therefore the detected current represents the integration of the spectral density of the 
current over $k$. Assuming a source with Gaussian spectral profile having center wavenumber $k_{0}$ and full width at half maximum (FWHM) $\Delta k$, the detected current integrated over the entire spectrum for a single reflection is given by [54]

$$
I_{D}\left(Z_{R}\right)=\frac{\rho}{4} S_{0}\left(R_{R}+R_{S}+2 \sqrt{R_{R} R_{S}} e^{\frac{-\left(Z_{R-} Z_{S}\right)^{2} \Delta k^{2}}{4 \ln (2)}} \cos \left(2 k_{0}\left(Z_{R}-Z_{S}\right)\right)\right.
$$

Where $z_{R}$ represents the arbitrary position of the reference mirror, $Z_{S}$ is the position of the reflector at the sample arm and $S_{0}$ is the spectrally integrated power of the source. Equation (2.4) shows that resultant signal is modulated by a cosinusoidal signal whose amplitude is proportional to the reflectivity of sample layer it decays with $e^{\frac{-\left(z_{R}-z_{S}\right)^{2} \Delta k^{2}}{4 \ln (2)}}$. This function is called as coherence gate function $[g(z)]$, which is directly linked with the power spectral density of the source, via Fourier transform. The detected signal is further filtered using a band pass filter, whose central pass band frequency matches with the frequency of the detected signal. The filtered signal is further demodulated using the envelop detection followed by the gray scale conversion (image construction).

\subsubsection{Fourier-domain OCT (FDOCT)}

Unlike TDOCT, the reference mirror of the interferometer is kept stationary in FDOCT systems and the depth information is retrieved from the spectral contents of the interferogram. Figure 2.3 shows that, for multiple reflectors, the resultant interferogram is a sum of the oscillations and the period of an oscillation (cosinusoid) is proportional to the path length difference between the reference mirror and the sample reflector. Therefore, depth reflectivity profile of the sample can be extracted from the interferogram using Fourier transform. This approach allows simultaneous acquisition of the signals from all 
the depth without the movement of the reference mirror. FDOCT is further classified into spectral-domain OCT (SDOCT) and swept source based OCT (SSOCT), depending on the scheme by which the spectral components are resolved and detected.

\subsubsection{Spectral-domain OCT (SDOCT)}

In SDOCT, the interferometer is illuminated by a broadband source and a spectrometer is used to separate the spectral components at the output. Figure 2.5 shows the schematic of a typical SDOCT system. The spectrometer typically consists of a grating which spatially resolves the spectral components, followed by the detection by a linear array of the photodetectors. Therefore the A-scan rate or the imaging speed is determined by the readout rate of the detector array. This approach was firstly used to measure the intraocular distances [67]. However, the first cross-sectional imaging using SDOCT system was demonstrated in 2002, by performing the in-vivo imaging of the retina [5].

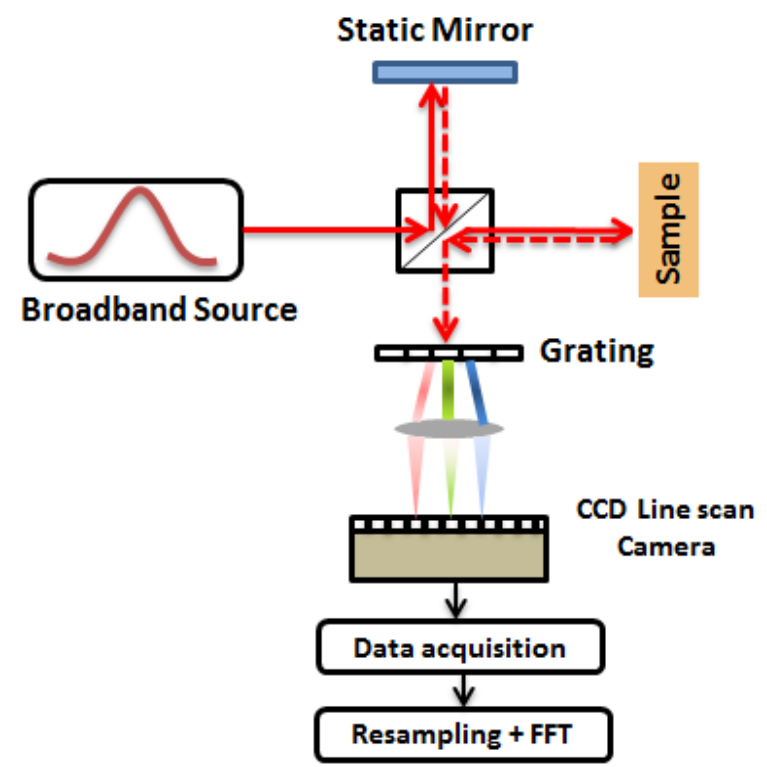

Figure 2.5 Schematic of spectral-domain OCT [50] 


\subsubsection{Swept source-based OCT (SSOCT)}

Figure 2.6 shows the basic setup of a SSOCT system. In a SSOCT system, a rapidly sweeping narrow bandwidth laser source is used for lighting the system and the spectral fringes are detected sequentially by a photodetector. The temporal detection of the spectral fringes is synchronized with wavelength sweeping. SSOCT system is also known as optical frequency domain imaging (OFDI) system. This technique, namely optical frequency domain reflectometry (OFDR) was used in the early 1980's for the measurement in optical fibers and fiber optic components [68]. The potential of this technique for tomographic imaging was firstly demonstrated by Fujimotto et al., in 1997 [10]. However, a SSOCT imaging with the improved quality and speed was firstly demonstrated in 2003 [6].

Consider a frequency swept laser source with initial wavenumber $k^{\prime}$, spectral width $\Delta k$, and sweeping period $\Delta T$. Assuming the laser exhibits a linear wavenumber-time characteristics, $k(t)=k^{\prime}+\frac{\Delta k}{\Delta T} t$. Considering the reflection from a single reflector, the detector current can be expressed as

$$
I_{D}(k)=\frac{\rho}{4} S[k(t)]\left(R_{R}+R_{S} \pm 2 \sqrt{R_{R} R_{S}} \cos (2 k(t) \Delta z)\right)
$$

This results in an oscillation with an angular frequency $\omega_{i}=2 \frac{\Delta k}{\Delta t} \Delta z$

This shows that the beat frequency generated by the interference of two delayed frequency sweeps is proportional to their path length difference. Therefore, a Fourier transform on the acquired fringe signal can construct the depth reflectivity profile of the sample. 


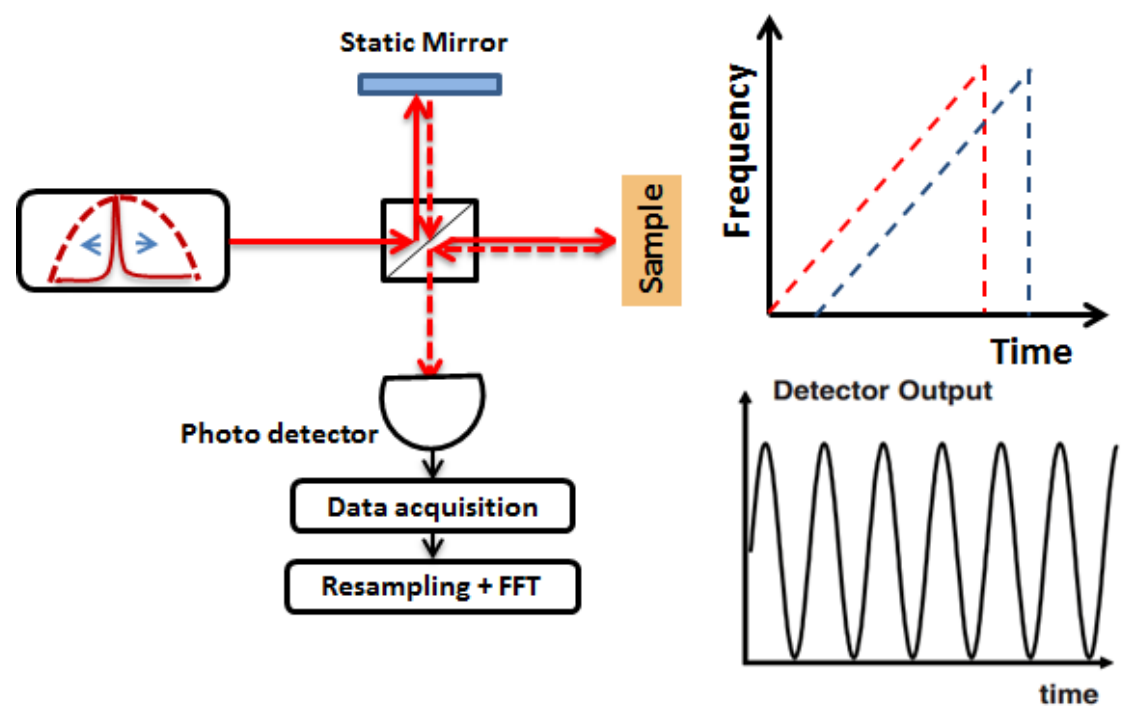

Figure 2.6 Schematic of swept source based-OCT system [50]

\subsection{Different fiber-optic implementations of OCT systems}

Fiber-optic implementations of OCT are seen as the most convenient for clinical applications, due to the compactness and flexibility. During the past years, there has been rapid development in the fiber-optic based technologies for frequency-domain and timedomain OCT systems. Different Interferometer configurations capable of providing highly compact and stable structure have been proposed and demonstrated [69-72]. Of these, standard Michelson interferometer (MI) has been widely used in OCT systems due to the easiness of implementation. Further, the single-mode fibers are generally used in these interferometric applications due to their advantage of guiding the light with minimal scattering into higher-order modes, preserving fringe contrast by ensuring mutual spatial coherence between the reference and sample light at the detector. Figure 2.7 shows the schematic of a typical SSOCT system using fiber-optic MI configuration. Here the light from the source is halved and fed into sample and reference arms by a 50:50 coupler. The light reflected from the reference and sample is recombined at the coupler and produce 
interference fringes. The reference arm of MI generally consists of a dispersion compensating glass, neutral density filter and a mirror as shown in Figure 2.7. The bulky and alignment sensitive reference arm affects the portability, stability and compactness of the OCT system.

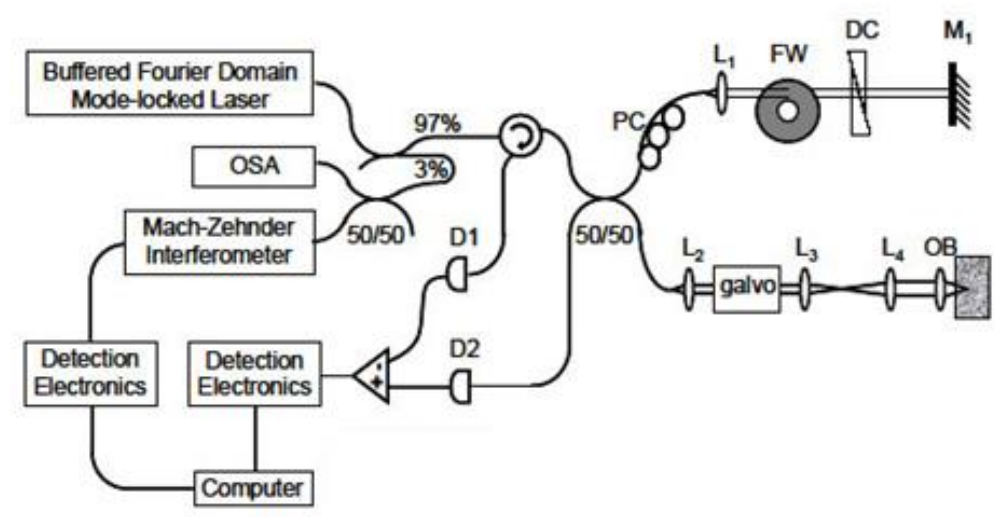

Figure 2.7 Schematic of a typical fiber-optic SSOCT system with MI configuration. L1-L4, lenses; M1, mirror; OB, water-immersion objective; D1-D2, dual-balanced photodetectors; FW, neutral density filter wheel; DC, dispersion compensating glass [73].

Alternatively, a common path interferometer (CPI) has been introduced for simplifying the system design and to improve the stability [74-78]. In CPI, the sample and reference arms share a common path as shown in Figure 2.8. Another significant advantage of the CPI is that the polarization and dispersion effects will be compensated automatically. The common path also improves the interferometer's stability and reduces its sensitivity to vibrations because beam overlap is automatically maintained. Accordingly, various timedomain and frequency-domain OCT systems based on CPI configurations have been demonstrated $[75,79,80]$. The main disadvantages of using common path system is the dependence of the fixed reference reflectivity where it is typically from the Fresnel (partial) reflection at the probe interface. Thus, for in situ or in vivo imaging, it would 
require dielectric or metal thin-film to obtain appropriate reference amplitude that is independent to the interfacial media.

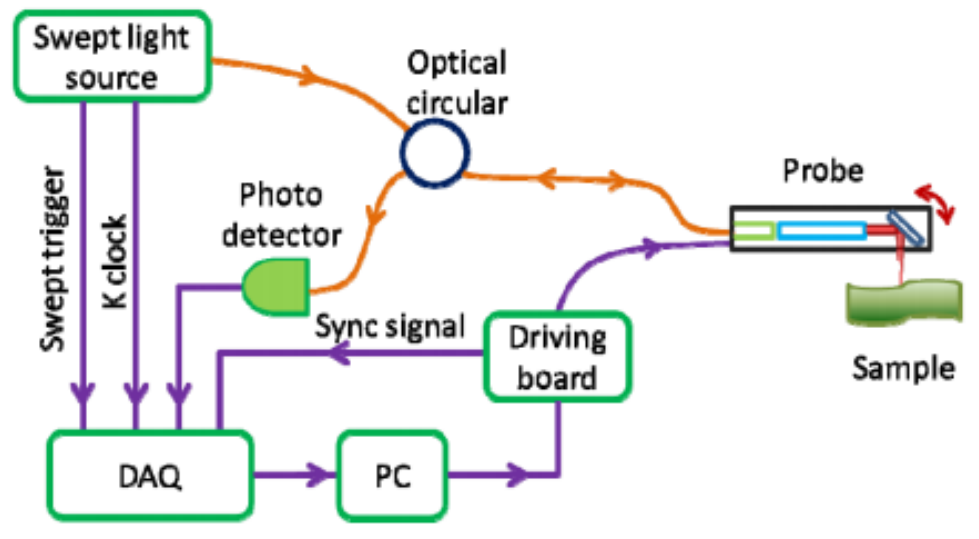

Figure 2.8. Schematic of SSOCT system with MEMS based common-path endoscopic imaging probe [76].

\subsection{Comparison of different OCT implementations}

Nowadays, research in the OCT regime focuses more on FDOCT systems. This is due to the two major advantages of FDOCT systems compared to TDOCT. The main advantage of the FDOCT systems is their high speed imaging compared to TDOCT. Typically, the imaging speed of an OCT is determined by its A-scan acquisition rate (or A-scan rate). In the case of TDOCT, the A-scan rate is limited by velocity of reference path length scanning. Therefore, imaging speed of the TDOCT is typically limited to only a few kHz. On the other hand, A-scan rate of the SDOCT depends on readout rate of the CCD, and sweep rate of the frequency swept laser source in the case of SSOCT. However, in any case, the imaging speed of FDOCT is much higher than (by a factor of 100 or more) that of TDOCT. Fast image acquisition allows the sampling of large volumes in a short time, which make FDOCT schemes highly desirable for in-vivo applications such as the retinal imaging and the intracoronary imaging $[81,82]$. Moreover, high speed imaging allows the 
temporally resolved observation of the fast transient processes in the developmental biology applications.

The other advantage of the FDOCT system is the high sensitivity compared to TDOCT. For the same incident optical power and the A-scan rate, sensitivity of the FDOCT is typically $20 \mathrm{~dB}$ to $30 \mathrm{~dB}$ higher than that of TDOCT [12]. This allows the FDOCT to detect considerably smaller back scattered signal, which further enhances the image quality. A high sensitivity is highly desirable in many imaging contexts such as the retinal imaging, where image quality is hindered due to the strong water absorption, and low scattering properties the retinal tissues. In such cases, OCT with high sensitivity can offer images with better quality, and major intraretinal layers can be distinguished. Similarly, difficulty in identifying the morphological differences between normal and neoplastic tissues prevents the detection of the diseases such as cancer at its earlier stages. This is mainly due to the reduced refractive index differences between normal and neoplastic tissues. Therefore OCT with higher sensitivity enables better delineation between the normal and diseases affected tissues. A high imaging sensitivity is also required in the developmental biology applications, where it allows imaging of the cells or organisms at the earlier stage of their development.

Nevertheless, FDOCT systems possess some disadvantages compared to TDOCT systems. The sensitivity roll-off with increasing imaging depth is one of the disadvantages commonly observed in all FDOCT systems. Similarly, resampling of the spectral fringes is required in most of the FDOCT systems. In TDOCT, imaging with high transversal resolution (higher NA) over large imaging depth can be achieved via the dynamic shift of 
the focal point coherently with path length scanning [83]. However, this approach is not applicable in FDOCT, since a single A-scan contains the complete depth information.

While comparing SSOCT with SDOCT, it is generally true to say that the fastest and higher quality imaging can be performed with SSOCT system. Moreover, the availability of the tunable laser with narrow spectral width such as the vertical-cavity surface emitting laser (VCSEL) in association with sophisticated data acquisition system offer deeper imaging with reduced sensitivity roll-off [13-15]. Compared to SDOCT, SSOCT allows low-cost detection at higher wavelengths, making it highly preferable for many diagnostic imaging applications. The signal detection with high sensitivity facilitated by the dual balanced detection scheme is another advantage in SSOCT systems. Similarly, SSOCT systems exhibit reduced fringe washout effect, compared to SDOCT systems. In FDOCT systems, the fringe signals are acquired over the integration time and the resultant signal will be an averaged signal. However, this averaging causes reduction in the amplitude and results in image distortion when there is a sample motion [84]. In SDOCT systems the signal averaging is performed simultaneously within integration time is equal to the A-scan period (T). Whereas, in SSOCT system each spectral channel is detected sequentially within the A-scan period. If there are $\mathrm{M}$ channels acquired in an $\mathrm{A}$-scan period $\mathrm{T}$, the integration time would be $\sim \frac{T}{M}$. Therefore, SSOCT exhibits negligible fringe wash out effect compared to SDOCT systems [85].

\subsection{Overview of biomedical applications of OCT}

OCT has recently emerged as a powerful tool in clinical medicine and biological research. Retinal imaging is one of the most clinically advanced applications of OCT. The high resolution retinal imaging with OCT allows efficient diagnosis of diseases like glaucoma, 
age-related macular degeneration [86]. Besides retinal imaging, OCT is widely used for imaging of anterior segment including cornea [87]. The wavelength regime used for anterior imaging is usually centered at $1310 \mathrm{~nm}$. Anterior chamber imaging is generally used for the measurement of thickness and refractive power of cornea and identification of causes for corneal opacity [88-90].

Another prominent application of the OCT is the intra coronary endoscopic imaging. Fiber-optic based OCT system integrated with imaging probes such as catheters or forward imaging probes are used for endoscopic applications [71, 91, 92]. Endoscopic OCT allows the detection of atherosclerotic plaques on the surface of arterial wall and efficient monitoring of stent deployment [91, 93]. The potential of endoscopic OCT for intravascular imaging was firstly demonstrated in 1996 [64]. Endoscopy OCT is also used in other medical contexts such as gastroenterology, pulmonary medicine, gynecology, and laryngology [94-99]. Endoscopic OCT allows the detection of superficial lesions and their quantification [100]. The OCT based real-time monitoring is widely used for better control of laser irradiation in laser ablation based treatment [101].

Besides the medical applications, potential of OCT has been explored in the field of developmental biology and cell biology [102, 103]. The label-free imaging with OCT allows the in-vivo investigation of the development of morphology and cell dynamics in their natural state. The higher temporal and spatial resolution of FDOCT systems, allows the efficient detection of cell migration and mitotic activity, which has a great impact in cell biology and medical applications such as the detection of cancer. 


\subsection{Point spread function and optical resolution}

\subsubsection{Axial resolution}

In OCT, the axial resolution is determined by the FWHM of the PSF. The PSF is defined as the OCT signal obtained by placing a mirror with reflectivity $R_{s}(\Delta z)=1$ at the sample arm. In the case of TDOCT, the PSF is represented by the envelope of the signal obtained after the demodulation. However, in FDOCT, the PSF is obtained by performing the inverse Fourier transform of the spectral fringes. In both techniques, the shape of the PSF is determined by the coherence gate function $g(z)$. Assuming that the spectral profile of the source has Gaussian shape with center wavenumber $k_{0}$ and spectral FWHM $\Delta k_{F W H M}$, then the spectral power density $\overline{S(k)}$ is given by [54]

$$
\overline{S(k)}=\frac{S}{\Delta k_{F W H m}} \frac{2 \sqrt{\ln 2}}{\sqrt{\pi}} e^{-\frac{4 \ln (2)\left(k-k_{0}\right)^{2}}{\Delta k_{F W H M}}}
$$

Where $S$ represents an arbitrary power. The Fourier transformation on the above equation yields the coherence gate function $g(z)$ as

$$
g(z)=S \cdot e^{-z^{2 \frac{\Delta k_{F W H M}}{4 \ln (2)}}}
$$

The FWHM of the coherence function $g(z)$, is given by [104]

$$
\Delta z_{F W H M}=\frac{4 \ln 2}{\Delta k_{F W H M}}=\frac{2 \ln (2)}{\pi} \frac{\lambda_{0}^{2}}{\Delta \lambda_{F W H M}}
$$

Where $\lambda_{0}$ and $\Delta \lambda_{F W H M}$ represents the center wavelength and the FWHM of spectral power density in terms of the wavelength. Equation (2.8) shows that the axial resolution of the OCT is inversely proportional to the bandwidth of the source. It is worth to mention that, if the measurement is performed in a medium with refractive index $n$, one has to 
consider the path length $n z$. Therefore, the effective axial resolution would be $\Delta z_{\text {eff }}=$ $\frac{\Delta z_{\text {air }}}{n}$.

\subsubsection{Transversal resolution}

Unlike confocal microscopy, the axial and the transversal resolutions in OCT are decoupled. In confocal microscopy, the transversal and the axial resolutions are determined by the diffraction limited focusing of the optical beam. However, this is applicable in OCT only for lateral resolution. The transversal resolution of the OCT is typically determined by the focal spot size of the imaging beam. For Gaussian beam illumination, the focal spot size $(\Delta x)$ is equal to twice the beam waist, and is given by [105]

$$
\Delta x=\frac{4 \lambda}{\pi} \frac{f}{D}=\frac{2 \lambda}{\pi N A}
$$

Where $D$ is the diameter of the beam spot incident on the objective lens, $f$ is the focal length and $N A$ represents the numerical aperture. Equation (2.9) shows that focusing with large $N A$ gives a reduced spot size, which consequently improves the transversal resolution. However this limits the usable depth range, defined as the confocal parameter (b). The confocal parameter, also known as the depth of field, is equal to twice the Rayleigh's range $\left(Z_{R}\right)$. The Rayleigh range is measured as the distance to the position where the beam spot size is increased by a factor $\sqrt{2}$, with respect to its focal spot size. The confocal parameter is related to beam spot size $(\Delta x)$ by the relation [105]

$$
b=\frac{\pi \Delta x^{2}}{\lambda}
$$


Typically, lower NA focusing is preferred in OCT systems in order to achieve maximum possible depth range. Moreover, this allows the transverse resolution nearly constant over the depth range. Similarly, imaging with higher transversal resolution can accomplished via optical coherence microscopy (OCM), where the objective with large NA is used for focusing. In OCM, the confocal parameter would be approximately equal to the axial $\operatorname{resolution}(b \approx \Delta z)$.

\subsubsection{Signal-to-noise ratio and Sensitivity}

The sensitivity of an OCT system is defined as the ratio of the incident signal power $\left(P_{s}\right)$ on the sample to the minimum detectable power $\left(P_{\min }\right)$ that is back reflected from a certain depth corresponding to a path length difference $2 \Delta z$. This is alternatively measured as the ratio of detector current $I_{D}(\Delta z)$ representing the peak of the PSF to the minimum detectable

current in the $I_{D, \min }(\Delta z)$. Since the detector current, $I_{D}(\Delta z) \propto \sqrt{P_{S}} \propto \sqrt{R_{S}}$, the sensitivity is directly linked to the minimum reflectivity $R_{S, \min }$. The logarithmic representation of the sensitivity is given by [54]

$$
\Sigma_{d B}=10 . \log \frac{P_{S}}{P_{\min }}=20 \log \frac{I_{D}(\Delta z)}{I_{D, \min }(\Delta z)}=10 \log \frac{1}{R_{S_{n}, \min }}
$$

The SNR is defined as the ratio of the amplitude of the maximum signal to the amplitude of the noise signal at a given point in time. The minimum detectable signal represents the minimum sample reflection intensity that gives rise to a signal whose amplitude is same as the noise level, or equivalently SNR is equal to one. The expression for SNR in electrical power is given by [54]

$$
S N R=\frac{I_{D}^{2}}{\sigma^{2}}
$$


Where the $I_{D}{ }^{2}$ and $\sigma^{2}$ represent the mean square peak current and variance of the noise, respectively. Therefore, the minimum detectable current is equal to the standard deviation of the noise floor $\left(I_{D, \min }(\Delta z)=\sigma(\Delta z)\right.$ ) for SNR is equal to one.

\subsection{Comparison of the sensitivities of different OCT configurations}

Table 1 given below compare the performance of the different OCT implementations in terms of mean square current, variance of the noise and, signal-to-noise ratio.

In the expressions, $S_{T D}$ and $S\left(k_{\mathrm{m}}\right)$ represent the incident optical power on the sample for TDOCT and FDOCT, respectively. Similarly, $B_{\mathrm{TD}}, B_{\mathrm{SD}}$ and $B_{\mathrm{SS}}$ represent the detection bandwidth for TDOCT, SDOCT, and SSOCT, respectively. $\rho$ and $M$ indicate the responsivity of the detector and number of spectral channels in FDOCT systems, respectively. In order to compare the sensitivities of different OCT configurations, optical power illuminating the sample $S$, A-scan acquisition time $T$, and the number of channels $M$ are assumed to be the same.

Table 2.1: Comparison of means square current, noise variance and SNR of three OCT implementations

\begin{tabular}{|c|c|c|c|}
\hline $\begin{array}{c}\text { OCT } \\
\text { Scheme }\end{array}$ & $\begin{array}{c}\text { Means square } \\
\text { peak current }\end{array}$ & Variance of noise & SNR \\
\hline TDOCT [1] & $\frac{1}{2} \rho^{2} \mathrm{~S}_{T D}^{2} \mathrm{R}_{\mathrm{S}} \mathrm{R}_{\mathrm{R}}$ & $\rho \mathrm{eS}_{\mathrm{TD}} \mathrm{R}_{\mathrm{R}} B_{\mathrm{TD}}$ & $\frac{\rho S_{T D} R_{S}}{2 e B_{T D}}$ \\
\hline SDOCT [5] & $\frac{M^{2}}{4} \rho^{2} S\left(k_{m}\right)^{2} R_{R} R_{S}$ & $M \rho e S\left(k_{m}\right) R_{R} B_{S D}$ & $M \cdot \frac{\rho S\left(k_{m}\right) R_{S}}{4 e B_{S D}}$ \\
\hline SSOCT [6] & $\frac{M^{2}}{4} \rho^{2} S\left(k_{m}\right)^{2} R_{R} R_{S}$ & $M \rho e S\left(k_{m}\right) R_{R} B_{S S}$ & $M \cdot \frac{\rho S\left(k_{m}\right) R_{S}}{4 e B_{S S}}$ \\
\hline
\end{tabular}


In SSOCT, the power used for illuminating the individual spectral channel is same as that of TDOCT $\left(S_{S S}\left(k_{m}\right)=S_{T D}\right)$. In the case of SDOCT system, the illumination power is contributed by all spectral channels; therefore, the optical power per spectral channel will be decreased by a factor M. i.e., $S_{S D}\left(k_{m}\right)=\frac{S_{T D}}{M}$. The detection bandwidth of SSOCT and SD OCT systems are $B_{S S}=\frac{M}{2 T}$ and $B_{S D}=\frac{1}{2 T}$, respectively.

The expressions for the SNR of SSOCT and SDOCT compared to TDOCT are given by[12]

$$
S N R_{S S O C T}=S N R_{S D O C T}=M \cdot \frac{\rho S\left(k_{m}\right) R_{S}}{4 e B_{F D}}=S N R_{T D O C T} \frac{M}{2}
$$

Equation (2.13) shows that SSOCT and SDOCT exhibit improved sensitivity relative to TDOCT system, by a factor $\frac{M}{2}$. The above SNR improvement is based on the assumption that the source has a rectangular spectral profile with same power distribution in all channels. However in practical, the spectral profile of the source can have different shape such as Gaussian, in which the SNR would be decreased again by a factor two or more. In any case, the availability of large number of spectral channels in practical swept sources and $\mathrm{CCD}$ arrays $(\mathrm{M} \approx 1000)$ offer an additional sensitivity of $20 \mathrm{~dB}-30 \mathrm{~dB}$ than TDOCT systems [12].

\subsection{Non-linear spectral sampling issues in OCT}

In FDOCT, high quality images can be constructed by performing fast Fourier transform (FFT) if the spectral signals are uniformly sampled in wavenumber domain. In most of the FDOCT systems, spectral fringes are recorded non-linearly in wavenumber domain [19, 106]. This is applicable for both SDOCT and SSOCT systems. In SDOCT systems, the 
spectral components are resolved linearly in wavelength by an optical grating. The nonlinear relationship between wavelength $(\lambda)$ and wavenumber $\left(k=\frac{2 \pi}{\lambda}\right)$ causes the spectral distribution unevenly in $k$-space. On the other hand, SSOCT systems make use of frequency $(k)$ swept laser sources and the spectral fringes are recorded as a function of time. However, most of the swept laser sources exhibit non-linear sweeping characteristic (wavenumber-time) that results in the acquisition of spectral fringes in non-uniform $k$ space. Therefore, in general, resampling of the spectral fringes into uniform $k$-space intervals is essential in FDOCT systems. In addition, most of the wavelength sweeping mechanisms employ mechanical movement or resonance, which further raises the concerns over the repeatability and stability of the spectrum. Thus, a real-time calibration is required for a high quality image construction.

Several methods have been demonstrated to resolve non-linear sampling issues in FDOCT systems. Hardware based external $k$-clock triggering methods have been used for calibration in SSOCT $[22,107]$. In this approach, electrical comb signals for the dynamic calibration of the swept spectra are generated using an external optoelectronics circuitry as shown in Figure 2.9.
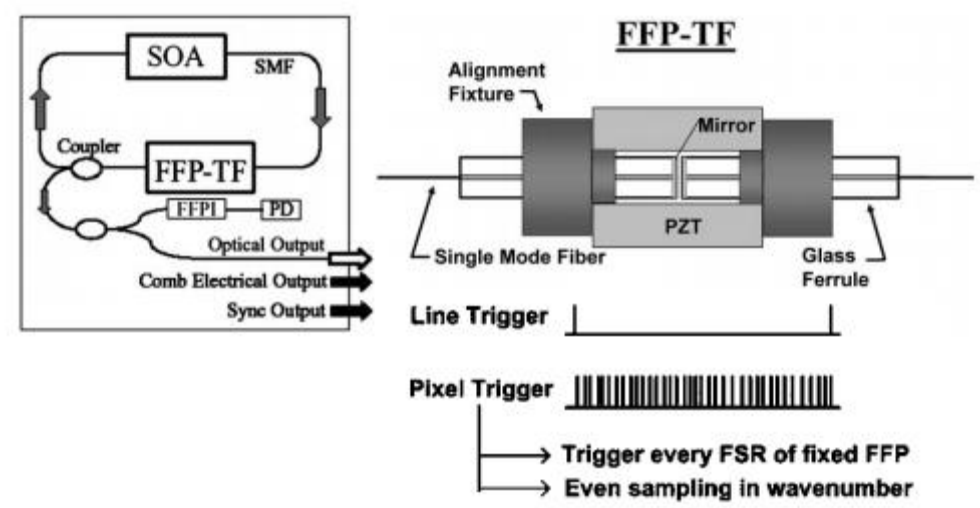

Figure 2.9 Fixed fiber Fabry-Perot interferometer (FFPI) and photodetector (PD) based trigger pulse generation scheme for uniform $\mathrm{k}$-space sampling [22]. 
The timing jitter associated with the clocking often lead to inaccurate resampling in these techniques. The intensity based calibration scheme that makes use of calibration signals from the auxiliary interferometers such as Fabry-Perot interferometer (FPI) and MachZehnder interferometer (MZI) have been demonstrated [23, 24]. In this scheme, time indices corresponding to the maximum and minimum values of the calibration signals are identified using a fast nearest-neighbor check algorithm. The inaccurate determination of peak and valleys points caused by the noise and quantization error often creates problems in these techniques. Alternatively, interpolation schemes based on FFT, zero-filling and cubic spline interpolation schemes in time-domain were demonstrated $[26,108,109]$. Of these, cubic interpolation offers better accuracy by compromising computational speed. Recently, phase analysis based calibration schemes have gained wide attention due to the fact that instantaneous phase of the fringe signal is directly proportional to the optical frequency. Accordingly, phase based k-domain interpolation, and time-domain interpolation based on polynomial fitting function have been demonstrated [25, 110]. The accuracy of the existing spectral-based time domain calibration schemes are limited due to errors associated with the polynomial fitting on the phase values. Higher accuracy can be achieved by fitting polynomials with higher orders. However, it would make the computation intensive and eventually reduce the speed. It is worth to mention that, all the aforementioned methods make use of external calibration devices such as interferometers and additional channel for data acquisition.

Alternatively, calibration schemes that do not rely on external devices are also reported in the literature. Linear-in-k spectrometers based on the specially designed prism as well as Fresnel zone plate were introduced for SDOCT systems [111, 112]. Similarly, k-linear swept sources based on Fourier Domain Mode Locking (FDML) and acousto-optic 
deflector were also introduced $[16,113]$. However, the complex and delicate hardware design in addition to the higher cost have made these approaches less attractive. On the other hand, numerical approaches based on non-uniform discrete Fourier transform (NDFT) and non-uniform fast Fourier transforms (NU-FFT), have been demonstrated [26, 114]. In the case of NDFT, a matrix called as Vander monde matrix, representing the wavelength distribution on the CCD plane is determined from an arbitrary spectral data. The depth information is retrieved by multiplying a pre-calculated Vander monde matrix with the acquired spectral data. Since the wavelength distribution with respect to the pixels positions is constant, this method is highly suitable for SDOCT systems. However, in the case of SSOCT, this matrix needs to be determined repeatedly due the inter-sweep variability. Moreover, NDFT based reconstruction is computationally complex than FFT computation, making them inappropriate for real-time applications. Recently, NU-FFT based image reconstruction has been demonstrated for FDOCT [114]. This approach is based on the grid computation by choosing Gaussian or Bessel kernel as convolution kernel. However this approach is feasible only with GPU architectures due to their computational burden and memory requirement.

\subsection{Nanoparticle assisted OCT imaging}

There has been increased interest in the research and development of various nanomaterials and structures for biomedical applications, particularly in the area of imaging and image-guided therapy. For most imaging modalities, effectiveness of the medical images can be enhanced with the aid of contrast agents. The agents selectively deposited at specific organ sites or tissues of interest to produce specific image signatures have been successfully utilized in almost every medical imaging technique, including 
ultrasound, CT, MRI, and optical microscopy. The contrast of conventional OCT is determined by the spatial variation of the tissue scattering, which is often affected by poor refractive index variation between the tissue layers and multiple light scattering. This limits the diagnostic capability of OCT in many bio-imaging scenarios. For example, imaging using OCT is useful for identifying normal and pathological tissues such as colon adenomas, actinic keratosis, and developing cardiac tissues [115, 116]. However, the image quality is often hindered by poor contrast between adjacent tissues. Similarly, identifying morphological differences between normal and neoplastic tissues at an early stage is difficult in OCT due to the reduced contrast [117]. This is true for OCT imaging, where the use of contrast agents offer the promise of enhanced diagnostic power with the potential to overcome the limitation of relying on inherent optical properties to discriminate pathology. The advent of novel bio-conjugated nanoplatforms has pushed the limits of molecular level to greater levels. Therefore, molecular information with enhanced sensitivity and specificity can be obtained from targeted site by attaching functional moieties to the surface of the specified nanoparticle. In addition to contrast enhancement, contrast agents are shown to be powerful multifunctional agents for drug delivery and photothermal therapy $[118,119]$.

Materials of various classes have been introduced to enhance the contrast in OCT imaging. The optical immersion liquids such as glycerol, propylene glycol, dextranes, and concentrated glucose solution have been used for contrast enhancement [120, 121]. However, the use of these immersion liquids decreases imaging sensitivity and affects the tissue's morphological appearance. Contrast enhancement using air-filled microbubbles, and engineered microspheres with a protein shell, and iron oxide microparticles have also 
been demonstrated for OCT imaging [122-124]. However, their large sizes $(>1 \mu \mathrm{m})$ prevent them from using in in-vivo imaging. Alternatively, NIR fluorescent molecules have been introduced for contrast enhancement in spectroscopic OCT [125]. Very fast quenching of the dye molecules and reduced imaging depth caused by absorption make this approach less attractive. The enhanced scattering and absorption properties caused by localized surface plasmon resonance (LSPR) in metal nanoparticles make them excellent candidates for various applications including contrast enhancement, drug delivery, and photothermal therapy $[33,126]$. Plasmonic nanoparticles possess potential capabilities in the field of optical imaging due to its large surface to volume ratio, tailorable size, shape and composition, structural robustness and its targeted binding capabilities. Owing to significant technological advancements in the synthesis, fabrication, and characterization of nanomaterials, it is now possible to tailor the size, shape and composition of the nanoparticle according to the specific imaging applications. Plasmonic nanoparticles synthesized from noble materials such as gold with or without combinations of a dielectric material are less toxic and are spectrally tunable. Accordingly, various gold nanostructures such as gold nanoshells, gold nanorods, and gold nanocages have been reported for contrast enhancement and photothermal applications in OCT around $800 \mathrm{~nm}$ window [127129]. OCT imaging around $1300 \mathrm{~nm}$ window have been widely attracted for many diagnostic imaging applications due to improved penetration in highly scattering tissues. On account of that gold nanorings and gold nanoshells have been demonstrated as contrast agents for OCT imaging around $1300 \mathrm{~nm}$. These nanoparticles show higher scattering to absorption cross-sections, thus predicting them as scattering based contrast agents. In general, very few nanoparticles and nanostructures have been reported for contrast enhancement in higher NIR wavelength imaging. 
In order to study the applicability and effectiveness of nanoprobes for diagnosis and therapy, their optical behavior, signal enhancement capability, and diffusion characteristics in biological tissues have to be measured in real-time. Different approaches have been reported for predicting the optical behavior of the nanoparticles in biological tissues. Simulations based on the Monte Carlo models were used to predict effect of nanoparticles on backscattered signals [130]. Conventional techniques based on integrating sphere and spectroscopy techniques have been widely used for characterizing the optical response of the nanoparticles [39, 41]. However, these measurements are not suitable for characterizing nanoparticles embedded in biological samples. The extinction coefficient of nanoparticles can be directly derived from the decay profile of OCT A-scan signals. In this method, A-scan signal is acquired from the nanoparticle solution followed by fitting an exponential function (or linear function in $\log$ scale) to the decay profile. The decay constant of the exponential function gives the extinction coefficient of nanoparticles. However, this approach is feasible only for the nanoparticles with high scattering crosssection. Moreover, a calibration function is needed in this approach for correcting the signal loss caused by the confocal effect [131].

The distribution of nanoparticles is another important factor that determines the effectives of the nanoparticle based diagnosis and therapy. For example, the adhesion of nanoparticles to cancer cells must be monitored in order to maintain the effectiveness of photothermal therapy. Similarly, if the affected area (lesion) is wide, the monitoring of a large distribution of nanoparticles is required. Different methods have been proposed for studying the Cell uptake and intercellular transport. A Brownian diffusion model has been demonstrated for studying the diffusion coefficient of gold nanoparticles in Zebrafish 
embryos [42]. Similarly, a two-dimensional mean square displacement (MSD) was demonstrated to track the trajectory of the silver nanoparticles in biological tissues [43]. These approaches were found to be successful only if the nanoparticle concentration is very low.

\subsection{Optical imaging for real-time monitoring of developmental biology}

Imaging has become a required methodology for developmental biologists for understanding the development and functions of microorganisms such as the bacteria. Biofilms are the aggregates of microorganisms such as the bacteria, which are adhered to living and inert surfaces [132]. They are generally enclosed in a matrix formed by extracellular polymer substances (EPS). Biofilms are widely observed in industrial and medical environments often giving rise to a wide range of concerns, especially biosafety [133]. The formation of the biofilm and EPS enable the microorganisms to achieve better resistance towards the antimicrobial treatment as well as tenaciously attaching to surfaces [134]. Hence it is often a challenge to eradicate biofilms from the biomedical and industrial environments in order to meet sufficient biosafety level. In order to have a better understanding and control of biofilms in highly sensitive environments, an efficient in-situ measurement/imaging scheme is required that would assist the strategies used for eradicating the biofilms.

Different imaging modalities have been reported for the investigation of biofilms. Nonoptical schemes such as the scanning transmission X-ray microscopy (STXM) and transmission electron microscopy (TEM) have been used for imaging biofilms [46]. STXM provides high resolution images up to $50 \mathrm{~nm}$ and allows mapping of chemical specious based on the chemical bonds. On the other hand, TEM provides a higher resolution up to 1 
nm and allows chemical identification when the sample is coated with gold-labeled lectins. Major disadvantage of these techniques is that they require sample preparation, which will alter the original structure of the biofilms. Moreover, these techniques use ionization radiation which affects the biofilm growth. Alternatively, nuclear magnetic resonance imaging (NMRI) has been used to image biofilm structure and study flow patterns [135]. However, the poor spatial resolution in addition to long acquisition time limits the applicability of NMRI in real-time imaging of biofilms. All these methods are generally bulky and need more space for their operation. This prevents them from using for real-time and in-situ investigations. Alternatively, optical techniques have been identified as promising tool for investigation of biofilms due to their potential for in-vivo and in-situ imaging using non-ionization radiation. Different optical schemes such as optical fluorometry, optical microsensor, Fourier-transform infrared spectroscopy (FTIR), and photoacoustic spectroscopy (PAS) have been proposed for monitoring of biofilms [136139]. The optical fluorometry and microsensor methods are used to map and quantify the photopigments and proteins via fluorescence measurement. Major limitations of these methods are the requirements of the staining and inapplicability in optically dense samples. Using FTIR and PAS, absorption spectra can be measured and biofilm growth can be monitored in a more specific fashion. In the case of FTIR, the penetration depth is limited to only a few microns, whereas PAS allows depth-resolved in-situ monitoring up to a few millimeters. This depth is approximately three orders of magnitude higher than that of FTIR. Nevertheless, the applicability of PAS is limited to biofilms growing on transparent substrata. Alternatively, optical microscopic techniques such as light microscopy and scanning confocal laser microscopy (SCLM) were introduced for high resolution imaging of biofilms [140, 141]. The SLCM provides high resolution enface images with improved 
contrast achieved via rejecting out-of-focus light [141]. This makes SCLM preferable to conventional light microscopy for the investigation biofilms. These enface images were then analyzed by post image-processing techniques to localize and quantify the cellular and non-cellular areas within the biofilm matrices. However, cell labeling requirements limit the applicability of CLSM for study of natural biofilm in-vivo. Similarly, the shallow imaging penetration limits its use for the investigation of thick biofilms. SCLM constructs the $3 \mathrm{D}$ images by the vertical movement of the focal plane. This limits the applicability of confocal microscopy for the real-time in-situ monitoring of biofilms. On contrary, OCT has potential to perform real-time in-situ monitoring of biofilms without staining [48, 142, 143]. OCT provides high axial resolution and sensitivity sufficient to resolve heterogeneous features in the biofilms. The near-infrared radiation used in OCT imaging allows the increased penetration through highly scattering structures. Accordingly, timedomain OCT systems with center wavelength around $800 \mathrm{~nm}$ have been used for imaging biofilms and their detachments [48]. Spectral-domain OCT system with high temporal resolution was used to visualize biofilm structure in shear stress experiments [49]. All these studies were performed on the biofilms with sufficient thickness and structural features. However, the limited axial resolution of the OCT often prevents it from imaging the biofilms in their earlier stages. This situation is more relevant in the case of planar biofilms such as Klebsiella Pneumonia (KP-1) which are generally developed in flat shape and without any distinct structures. In addition, the real-time in-situ quantification of the microbial communities has great importance in developmental biology research as well as in their eradication process. 


\subsection{Outcome of Literature Review}

The research and development of accurate, compatible, and low cost analytical/diagnostic tools is one of the growing areas is the biomedical industry. OCT has emerged as one of the powerful biomedical optical imaging modality with depth-sectioning ability down to micrometer-scale. Compared to the standard clinical imaging modalities such as CT and PET, OCT is contact free and does not use ionization radiation. Further, OCT provides an resolution close to the histopathology [3]. Imaging at higher wavelength window such as $1300 \mathrm{~nm}$ reduces the background scattering and improves the tissue penetration. The high speed and sensitivity of FDOCT systems has improved its diagnostic potential in ophthalmology, cardiology, and dermatology. Review on recent trends in OCT imaging shows that SSOCT is a better choice for many diagnostic applications because of its high speed imaging, reduced sensitivity fall-off, and capability of low cost detection at higher wavelength $[13,14]$.

The fiber-optic implementation of OCT not only allows high contrast imaging but also provides excellent compactness and stability for OCT configuration [79, 144]. Different fiber optic interferometers have been introduced for OCT applications. Among these, common path interferometer (CPI) has been widely attracted due to its compact structure. However, the poor tunability of the reference arm power reduces the imaging sensitivity in CPI systems. Fiber-optic Michelson interferometer is widely used in OCT systems due to the easiness of implementation. However, the compactness, stability and portability of Michelson interferometer are often challenged by the bulky and alignment sensitive reference arm. Literature shows that the research is still focused on developing compact and stable interferometer design that can facilitate high performance imaging. 
The resampling scheme used in SSOCT system has a great impact on its axial resolution and sensitivity $[17,19]$. Inaccurate resampling compromises the performance in terms of ranging accuracy and sensitivity, and results in images with poor quality. The review on different calibration schemes shows that a real-time calibration is always preferred in SSOCT systems $[26,113]$. The performance of existing calibration schemes is limited in terms of accuracy and speed due to the higher order polynomial fitting and other iterative operations used in their algorithms [25]. Moreover, most of the schemes rely on additional hardware resources for calibration signal generation and acquisition. This makes the system more complex and expensive. On the other hand, software based approaches allow calibration without the need of any external devices. However, they are computationally intensive and not feasible for real-time calibration in SSOCT systems. This shows that there is a great demand for a high performance real-time calibration scheme without increasing hardware and computational complexity.

The contrast enhancement in OCT systems using different exogenous contrast agents has been widely studied by different research groups [120-128]. The unique optical response of plasmonic nanoprobes in terms of their enhanced scattering and absorption has made them powerful contrast agents for imaging and photothermal applications. Literature highlights that studies on nanoparticle based contrast enhancement are limited to only lower imaging window. The current approaches used for the estimation of extinction properties are more feasible for nanoparticles with higher scattering cross-sections. The inaccuracies associated with exponential fitting and requirement of an additional calibration function are the other issues with these techniques. This point towards the requirement of a generic approach that accurately measures the optical extinction properties of scattering as well as absorption 
nanoparticles. Literature also highlights the requirement of an efficient monitoring scheme for nanoparticle distribution and their transport in biological tissues over large area.

The in-vivo analysis of the development of bacterial biofilms has considerably evolved during the last decade, in line with technical advances in microscopy. The bacterial biofilms are widely found in domestic, industrial, and medical environment and identified as the major reason for the inflectional diseases $[132,145]$. The review on the different monitoring schemes shows that optical imaging and measurement techniques are more feasible for in-vivo or in-situ investigations of biofilms [45-48]. Optical methods allow high resolution imaging of biofilms using non-ionization radiation. The introduction of the confocal microscopy has led to a considerable progress in studying the architecture and physiology of the biofilms. However, the limited penetration depth and requirement of the fluorescent labeling are the major disadvantage of this approach. On the other hand, OCT has been emerged as a powerful tool for investigation of the natural biofilms because of its capability to perform label-free imaging at high resolution and better imaging depth [48, 49]. Further, fast acquisition rate of OCT allows real-time in-situ imaging and quantification of biofilms.

Next chapter details the design and development of a compact SSOCT system with improved imaging capabilities. The characterization aspects of SSOCT system and relevant details, which actually led to establishment of a novel configuration and enhanced imaging capability, will also be elaborated. 


\section{Chapter 3: A compact SSOCT system with enhanced imaging capability}

This chapter gives a comprehensive analysis of an instrumentation scheme for the development of a novel swept-source based optical coherence tomography system (SSOCT). The experimental investigations are fundamentally directed towards establishing a compact SSOCT system with enhanced imaging capability. This chapter begins with a detailed description of the fundamental theoretical concepts and various design considerations of the SSOCT imaging. The instrumentation scheme involving optoelectronic hardware design and advanced signal processing schemes are illustrated by highlighting their technical relevance and measuring challenges. The imaging capability of the developed system is determined analytically and experimentally. This chapter concludes with the results obtained from the in-vivo imaging of different biological samples.

\subsection{Introduction}

Optical coherence tomography (OCT) has emerged as one of the most successful applications of interferometry in biological sciences. The ability of low coherence interferometry to perform optical ranging measurements with micrometer resolution and millimeter penetration allows anatomical, structural and functional mapping of biological samples $[3,54]$. Frequency-domain OCT using swept-source technology allows highly efficient imaging in terms of sensitivity and speed. The design and development of a high performance SSOCT system is generally a challenging task due to its modular structure. 
From a system point of view, OCT is a combination of different integrated hardware and software modules with different functionalities, as shown in Figure 3.1. The major modules in the system include the light source, optical interferometer, beam delivery system, data acquisition and computer control, and data and image processing.

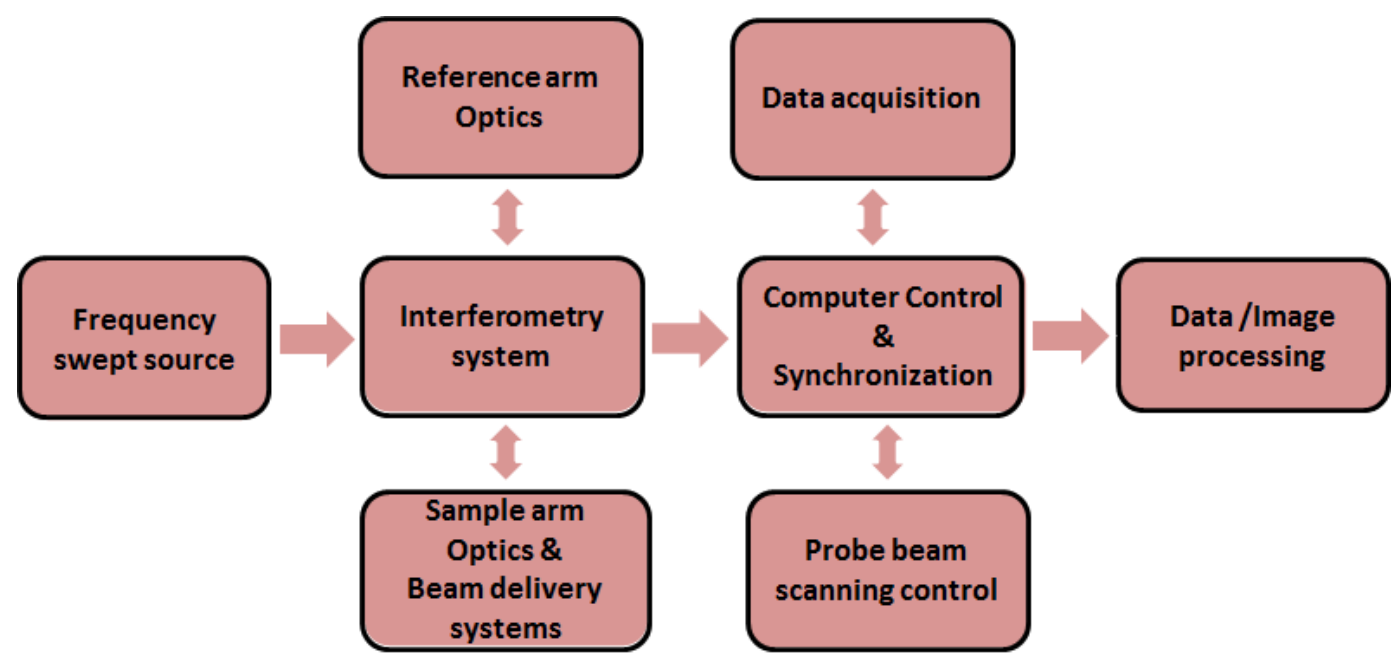

Figure 3.1 Modular structure of a SSOCT system

The individual components and functional modules play a critical role in determining the performance of OCT system. These individual modules are cascaded in sequential, each contributing a transfer function that will combine to form the ultimate system response. Therefore, the selection of components and their integration based on theoretical requirements and various design considerations are paramount in SSOCT design.

The following sections outline the fundamental theoretical concept that governs the imaging capabilities of SSOCT and its various design considerations. The instrumentation scheme of the proposed SSOCT system was designed, configured and characterized based on these theoretical concepts. 


\subsection{Fundamental theoretical concepts of SSOCT}

The principle of SSOCT relies on the spectral interferometry in which a narrow band light is rapidly tuned over a broad bandwidth and spectral fringes are sequentially collected by a single photodetector. The spectral interference signal representing the reflection from a single reflector can be mathematically represented by Equation (2.2)

$$
I_{D}(k)=S(k)\left[R_{R}+R_{S} \pm 2 \sqrt{R_{R}} \sqrt{R_{S}} \cos (2 k \Delta z)\right]
$$

According to the Wiener-Khinchine theorem, the spectral density function $I(k)$ is the Fourier transform of the auto-correlation function. An inverse Fourier transform is performed to retrieve the reflectivity profile of the sample. The depth reflectivity can be expressed as [146]

$$
\begin{gathered}
I_{D}(z)=F T^{-1}\left[I_{D}(k)\right]=F T^{-1}[S(k)] \circledast F T^{-1}\left[R_{R}+R_{S} \pm 2 \sqrt{R_{R}} \sqrt{R_{S}} \cos (2 k \Delta z)\right] \\
I_{D}(z)=F T^{-1}[S(k)] \circledast\left[\delta(z)\left(R_{R}+R_{S}\right) \pm 2 \sqrt{R_{R}} \sqrt{R_{S}}(\delta(z+\Delta z)+\delta(z-\Delta z))\right]
\end{gathered}
$$

where $\circledast$ represents convolution operation.

The coherence gate function $F T^{-1}[S(k)]$ can be denoted by $g(z)$, and Equation (3.2) simplifies to:

$$
I_{D}(z)=g(z)\left[R_{R}+R_{S}\right] \pm \sqrt{R_{R} R_{S}}[g(z+\Delta z)+g(z-\Delta z)]
$$

The resultant signal has DC terms at $\mathrm{z}=0$ and interferometric terms which are identical and symmetric with respect to $\mathrm{z}=0$ as shown in Figure 3.2. The DC term is mainly contributed by the reference arm power and appears as artifacts in the image. In the case of multiple reflectors, the autocorrelation term will also results in artifacts near to the zero path length point. In practical OCT systems, artifacts due to the DC term will be removed 
via background subtraction. The effect of autocorrelation is mitigated by controlling the reference arm power such that the cross-correlation term has higher amplitude than the autocorrelation.

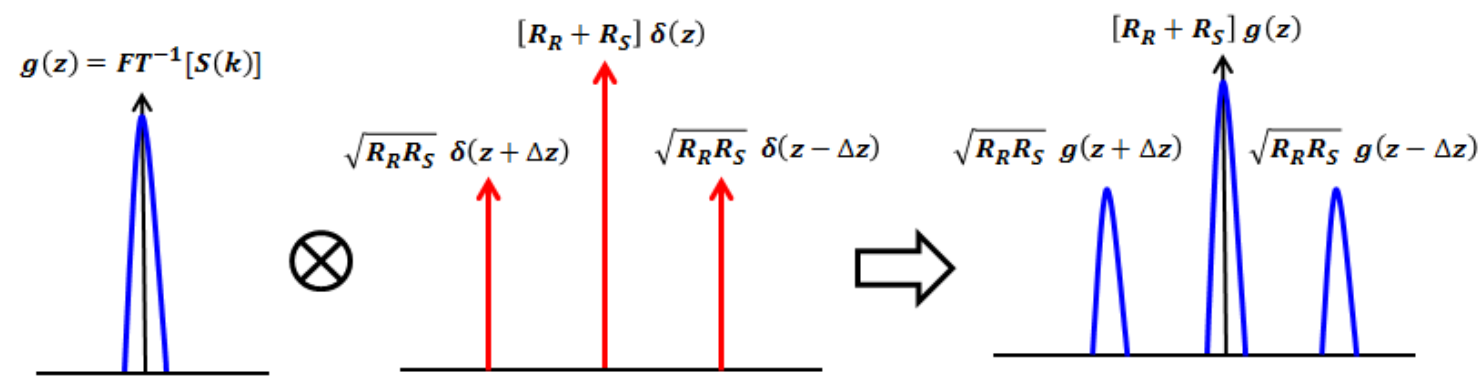

Figure 3.2 Signal reconstruction in SSOCT systems

The symmetrical terms with respect to zero plane $(\mathrm{z}=0)$ appears as mirror images and they overlap each other if sample strays over to zero path length point. This is known as the complex conjugate artifacts in SSOCT systems. Overlapping of the imaging can be avoided by keeping the sample completely at the one side of the zero path length plane.

In SSOCT, the spectral interferogram data is sampled by a spectral resolution function defined by the instantaneous spectral width of the laser source. The appropriate expression for the acquired spectral fringes is then obtained by convolving the spectral resolution function with the ideal spectral interferogram given in Equation (2.2). This is equivalent to multiplying Fourier transformation of spectral resolution function with detected signal in the spatial domain $\left(I_{D}(z)\right)$. Assuming a Gaussian spectral resolution function $\psi(k)=$ $e^{-\frac{4 \ln (2) k^{2}}{\delta_{r} k^{2}}}$ centered at $k=0$ and instantaneous spectral width $\delta_{r} k$. The resultant signal can be expressed as [54]

$$
I_{D}(k) \circledast e^{-\frac{4 \ln (2) k^{2}}{\delta_{r} k^{2}}}=I_{D}(z) e^{-\frac{z^{2} \delta_{r} k^{2}}{4 \ln (2)}}
$$


This results in the decay of the amplitude of the point spread function with distance, as shown in Figure 3.3. This is an inherent feature observed in SSOCT system, known as sensitivity roll-off. The fall-off sensitivity with depth is observed as the decrease in the fringe visibility of the spectral fringes at higher imaging depths. Thus, the sensitivity rolloff has a great impact on the imaging depth and it is characterized in terms of principal imaging range. Principal imaging range is measured as the depth at which the peak sensitivity is reduced by $-6 \mathrm{~dB}$. This shows that a source with finer spectral resolution function can broaden the envelope and reduce the sensitivity roll-off rate. For a spectral resolution function with Gaussian profile, the principal imaging range $Z_{-6 d B}$ is given by [104]

$$
Z_{-6 d B}=\frac{2 \ln (2)}{\pi \delta_{r} \lambda}=\frac{\ln 2}{\pi} \frac{\lambda_{0}^{2}}{\delta_{r} \lambda}
$$

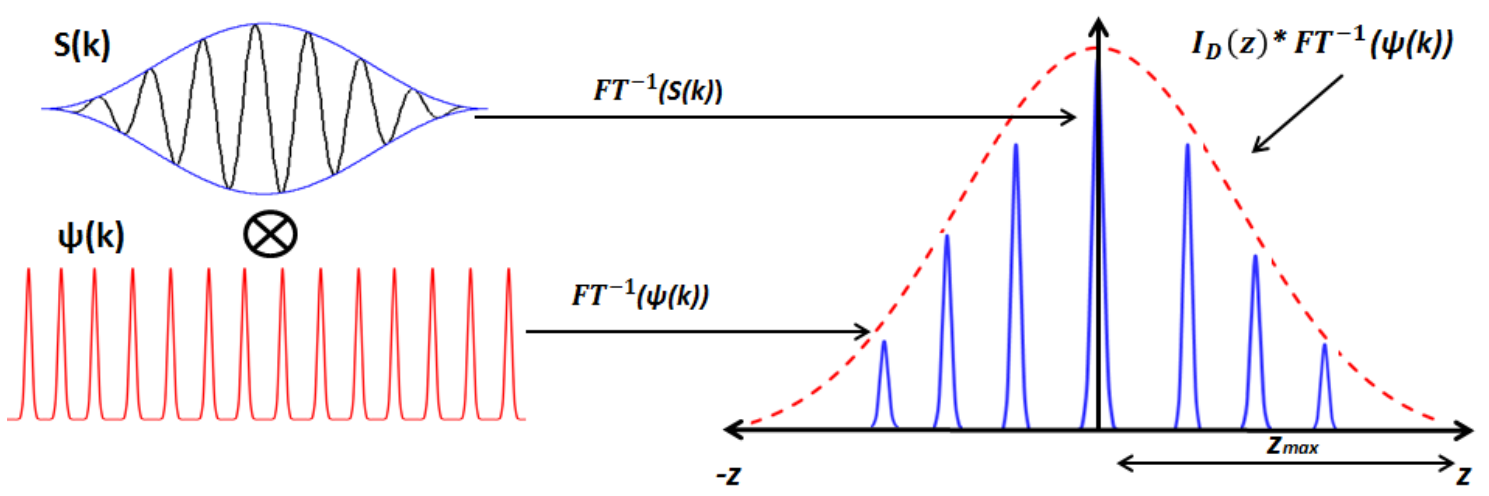

Figure 3.3 Representative figure showing the decay of the amplitude of the PSF with depth

Similar to the spectral sampling, digital sampling of the spectral fringes also has major consideration in the practical implementation of SSOCT. Typically in SSOCT system, the photo detected signal is digitally sampled by a high speed data acquisition system (digitizer) at a high sampling rate. The sampling rate or number data samples determine the 
maximum imaging depth $\left(Z_{\max }\right)$. Assuming that the interferogram is linearly sampled at an interval $\delta_{s} k$ over a spectral period $\Delta k$ results in $\mathrm{M}$ number of samples such that $\Delta k=M \delta_{s} k$. This gives a sample interval of $\frac{\pi}{\Delta k}$ in spatial (z) domain, after the discrete Fourier transformation. Therefore, the total range in z-domain is equal to $\frac{\pi}{\delta_{s} k}$. Therefore the maximum imaging depth is given by [147]

$$
z_{\text {max }}= \pm \frac{\pi}{2 \delta_{s} k}= \pm \frac{\lambda_{0}^{2}}{4 \delta_{s} \lambda}
$$

The following sections detail the system configuration methodologies and its implementation based on the above mentioned theoretical concepts and design considerations. Various advanced signal processing routines are used to formulate the configuration schemes for the proposed SSOCT system relevant to this research.

\subsection{Instrumentation scheme for the proposed SSOCT system}

Technically, OCT is a hybrid system comprising of various optical, electronics, and electro-mechanical functional modules. The schematic of the proposed compact SSOCT imaging setup is shown in Figure 3.4. The major integral hardware schemes in the SSOCT design include fiber-optic interferometer, laser source and optical assemblies, data acquisition, and control signal generation for laser scanning and synchronization. A highly compatible and stable configuration is accomplished by implementing two novel approaches in the configuration: (1) choosing a non-reflective arm based interferometer configuration; and the (2) implementation of an automatic calibration scheme that does not rely on auxiliary interferometer for calibration signal generation and additional channel for 
recording the signal. Descriptions of the individual hardware modules are given in the following subsections.

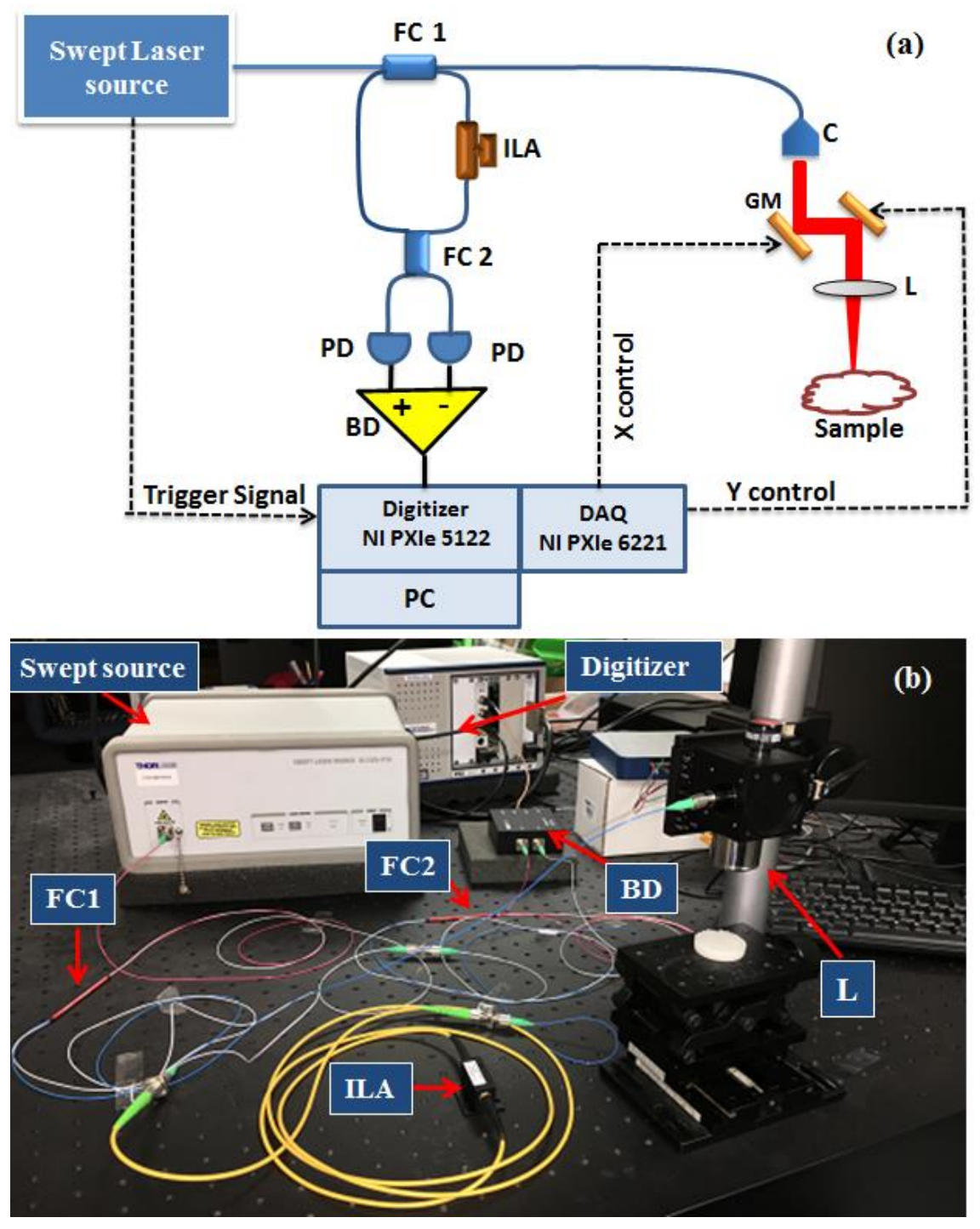

Figure 3.4 (a) Schematic of the developed SSOCT system (b) Photograph of the developed system. BD: Balance detector; C: Collimator; DAQ: Data acquisition and Control; FC: Fiber coupler; GM: Galvo Mirror; ILA: In-line variable Attenuator; PC: Personal Computer; PD: Photodetector.

\subsubsection{Frequency-Swept Laser Source}

The frequency swept-laser source is the key module in SSOCT systems and it determines the major imaging parameters such as axial resolution, speed, penetration depth, and sensitivity roll-off rate. This proposed SSOCT system consists of a high-speed frequency- 
swept external cavity laser source (SL1325-P16, Thorlabs, Germany), which has a central wavelength of $1320 \mathrm{~nm}$ and sweeping range of $100 \mathrm{~nm}$ as shown in Figure 3.5. The source has a repetition rate of $16000 \mathrm{~A}$-scans/second and delivers an average optical power of $12 \mathrm{~mW}$. The instantaneous linewidth of the laser is found to be $0.290 \mathrm{~nm}$, and has a coherence length of $6 \mathrm{~mm}$. The comprehensive details of architecture of swept laser source SL1325-P16, used in the imaging system is given in the Appendix A.

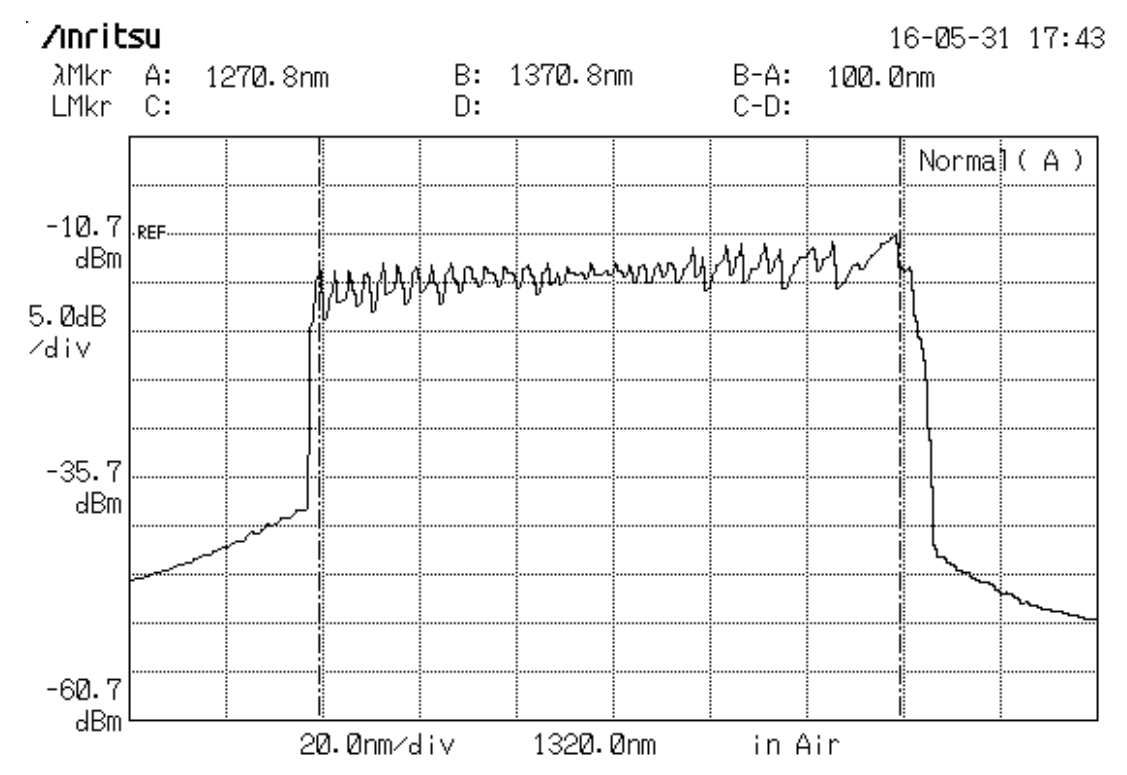

Figure 3.5 Spectrum of the SL1325-P16 swept laser acquired using optical spectrum analyzer.

\subsubsection{Non-reflective reference arm-based Interferometer configuration}

A novel interferometer configuration based on non-reflective arm is implemented in the proposed SSOCT system as shown in Figure 3.4. The output light from the swept source laser is launched into a 75/25 wideband fiber-optic coupler (FC1) (TW1300R3A2, Thorlabs, USA). $75 \%$ of the power is directed to the sample arm of the interferometer. The remaining $25 \%$ is directed to another fiber coupler (FC2). This length of fiber carries the reference signal for the interference. The length of the fiber has to be well-matched to path lengths of the two interfering beams. An in-line variable fiber optical attenuator (VOA50, 
Thorlabs, USA) is used to control the reference signal intensity. This approach avoids the use of neutral density filter (ND) and the bulky and alignment sensitive mirrored reference arm. The reference arm power is optimized using the in-line variable fiber optical attenuator to achieve maximum sensitivity. Back-reflected light from the sample interferes with the forward reference signal at FC2. The resultant fringe signals are acquired using a dual balanced detection (BD) scheme. The optical fiber used in the interferometer has average mode field diameter of $9.2 \mu \mathrm{m}$, cladding diameter of $125 \mu \mathrm{m}$, and numerical aperture of 0.14 .

\subsubsection{Balanced detection and data acquisition}

The balanced detection (PDB440C, Thorlabs, USA) improves the signal-to-noise ratio by suppressing the relative intensity noise (RIN) and auto-correlated noise present in detected signals. It consists of a pair of well-matched photodetectors (PD) and a low-noise, highspeed current-to-voltage converter (transimpedance amplifier) that produces an electrical voltage proportional to the differences between input photocurrents. The subtraction of detected signals in out-of-phase mode, results in the cancellation of common mode noise. The balance detected OCT signals are digitally sampled using a high speed digitizer (PXIe NI-5122, National Instruments) at a sampling rate of $50 \mathrm{MHz}$ and 14 bit resolution. This corresponds to 3125 data points for a single sweep of the laser. Among the 3125 points, only 2800 samples carry relevant spectral information. Thus the acquired data points are truncated to 2800 and used for further processing. The sampling rate is chosen in such a way that the sampling interval is smaller than instantaneous linewidth. Otherwise the sensitivity will fall rapidly with the depth. The data acquisition is synchronized with the laser sweeping via A-scan trigger signal from the source. The digitally sampled fringe data 
is temporarily stored in the onboard memory of the digitizer, and transferred to a work station personal computer (PC) (Dell Precision T1650) upon receiving desired number of A-scan trigger.

\subsubsection{Beam delivery system and control signal generation}

Galvanometer based mirrors and a telecentric objective at the sample arm optics allows the efficient delivery of probe beam into the sample. Figure 3.6 shows a schematic of the sample arm optics containing galvo mirror based beam steering setup and telecentric objective.

(a)

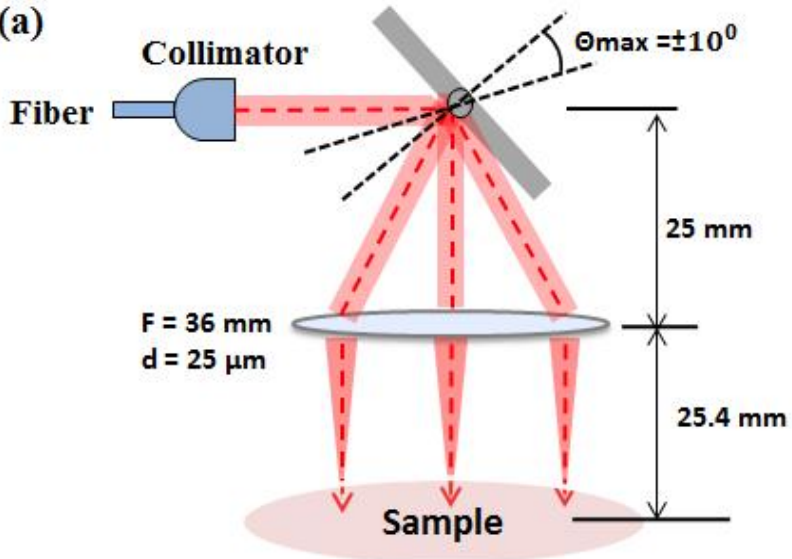

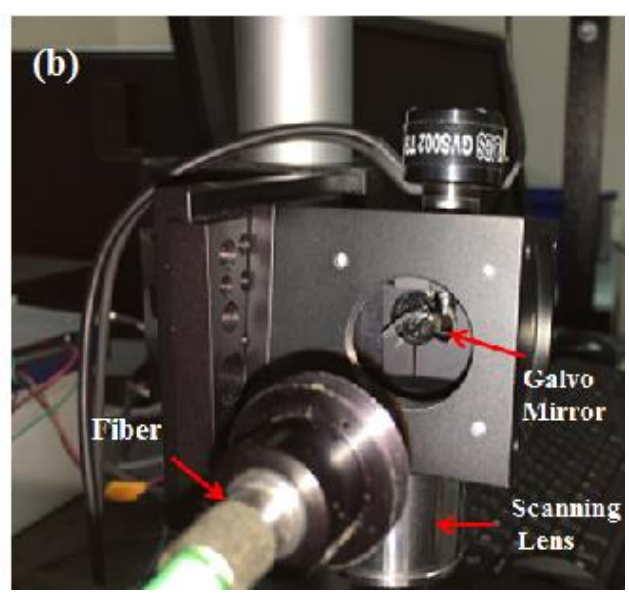

Figure 3.6 (a) Schematic of sample arm optics (a) Photograph of the sample arm optics used in the system

The developed system uses two orthogonally aligned galvo mirrors to scan the laser beam over the sample in $\mathrm{x}$ and $\mathrm{y}$-directions. Light from the sample arm is collimated prior to the scanning. The reflected beam from galvo mirrors is focused onto the sample surface by a telecentric lens (LSM 03, Thorlabs, USA).

A low-speed analog card (DAQ) (NI PXI-6221, National Instruments) synchronized with the data acquisition unit is used to control the motion of the galvo mirror. Control signal 
(voltage waveform) generated by the analog card is fed to the driver circuit. The galvo system is calibrated such that a voltage swing between $\pm 10 \mathrm{~V}$ is linearly transformed into an angular deviation of \pm 10 degree. Consequently, an input triangular waveform is used to drive the galvo mirror linearly to and fro across the zero mean position (0V). Figure 3.7 illustrates the triangular waveform used for controlling the galvo mirror. The scanning range is regulated by the software control. The increment in steps is synchronized with the trigger signal from the swept laser source. One period of the triangular wave represents two frames. The voltage sweep from $-10 \mathrm{~V}$ to $+10 \mathrm{~V}$ represents ( $\mathrm{AB}$ in Figure 3.7) a forward scan, whereas the reverse sweep from $+10 \mathrm{~V}$ to $-10 \mathrm{~V}$ results in a backward scan (BC in Figure 3.7).

The analog card has an onboard 16 bits digital to analog converter (DAC), which gives a voltage resolution (lowest incremental voltage) of $0.305 \mathrm{mV}$. This corresponds to an angular deviation of 0.0003 degree. The focusing optics of the SSOCT system has a mean spot size (1/ $\mathrm{e}^{2}$ beam diameter in the field of focus) of $25 \mu \mathrm{m}$, and effective focal length of $\sim 36 \mathrm{~mm}$. This gives the required minimum angle of rotation of 0.0198 degree by the relation[148]

$$
\theta=\frac{1}{2} \tan ^{-1}\left(\frac{\text { spot size }}{\text { focal length }}\right)
$$

The angular resolution achievable with the proposed configuration is approximately three orders of magnitude higher than the required minimum angle of rotation. Therefore, the developed configuration allows highly stable and precise beam positioning with high density sampling. All the software control for waveform generation and synchronization are performed in LabVIEW software on real time basis. 


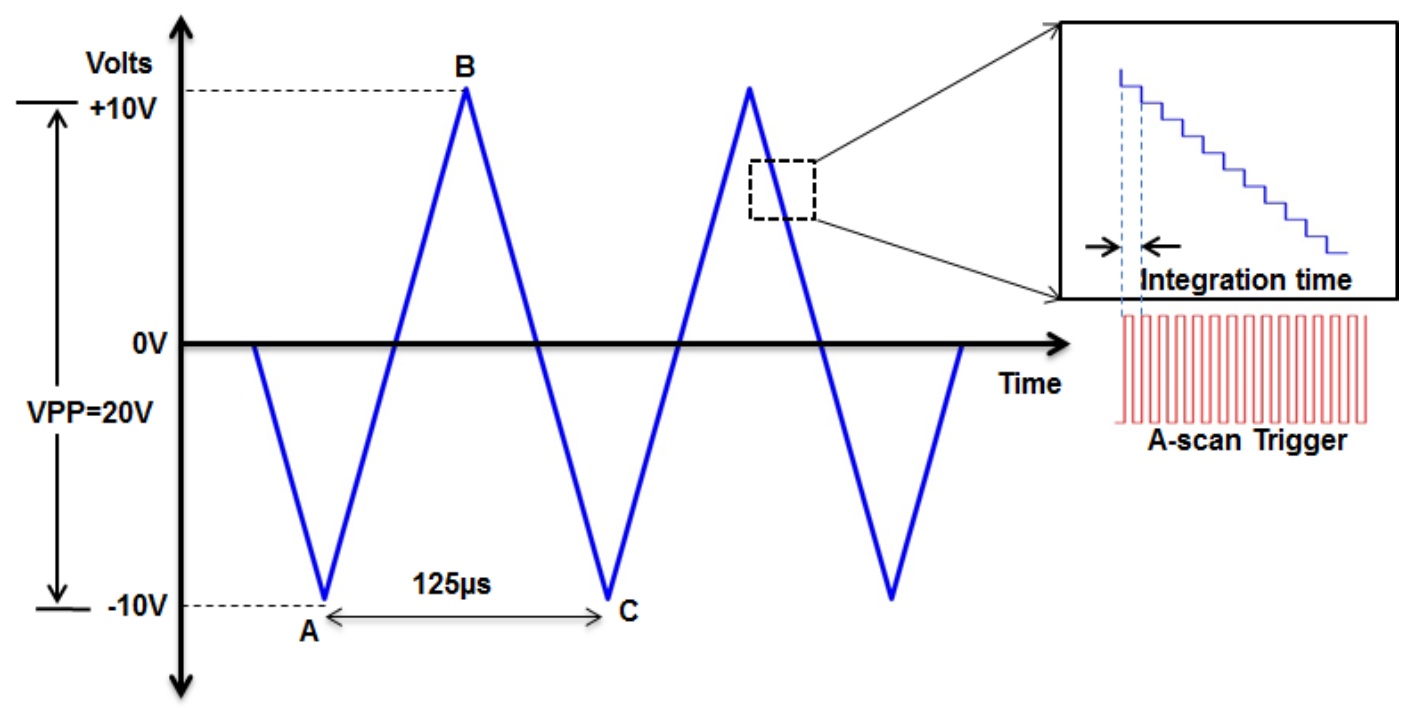

Figure 3.7 Control waveform for galvo mirror based beam steering. AB: forward scan BC: backward scan

\section{4 Implementation of advanced signal processing routines}

Compared to time-domain OCT (TDOCT), Fourier-domain OCT schemes are more computationally intensive as it requires many signal processing stages to construct the image. The major signal processing schemes involve background subtraction, dispersion compensation, spectral apodization, wavenumber calibration and resampling of OCT signal, and inverse FFT followed by the compression and gray scale conversion. Figure 3.8 illustrates the process flow of major signal processing steps required to create depth resolved image from OCT spectral signals. 


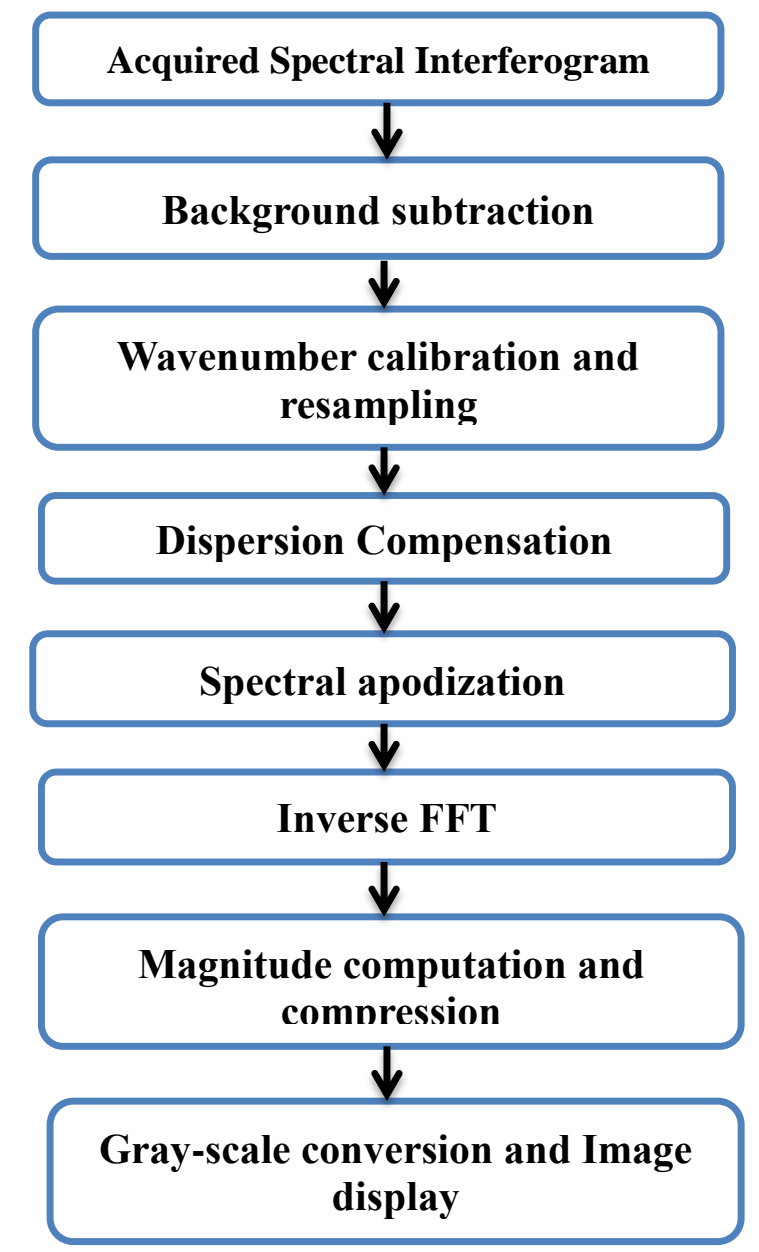

Figure 3.8 Process flow of signal processing steps in SSOCT

\subsubsection{Background subtraction}

Background subtraction involves the recording of the signal by blocking the sample arm prior to every image acquisition, and subtracting with the subsequent interference signals. The signal acquired by blocking the sample arm would contain the residual signal from the reference arm and the signal generated by the imperfect symmetry of the balanced detector. Background subtraction eliminates the DC component and residual signals present in the OCT signal. This helps to remove artifacts and improve the contrast of 
image. Figure 3.9 illustrates the impact of the balanced detection followed by background subtraction on a spectral interferogram.
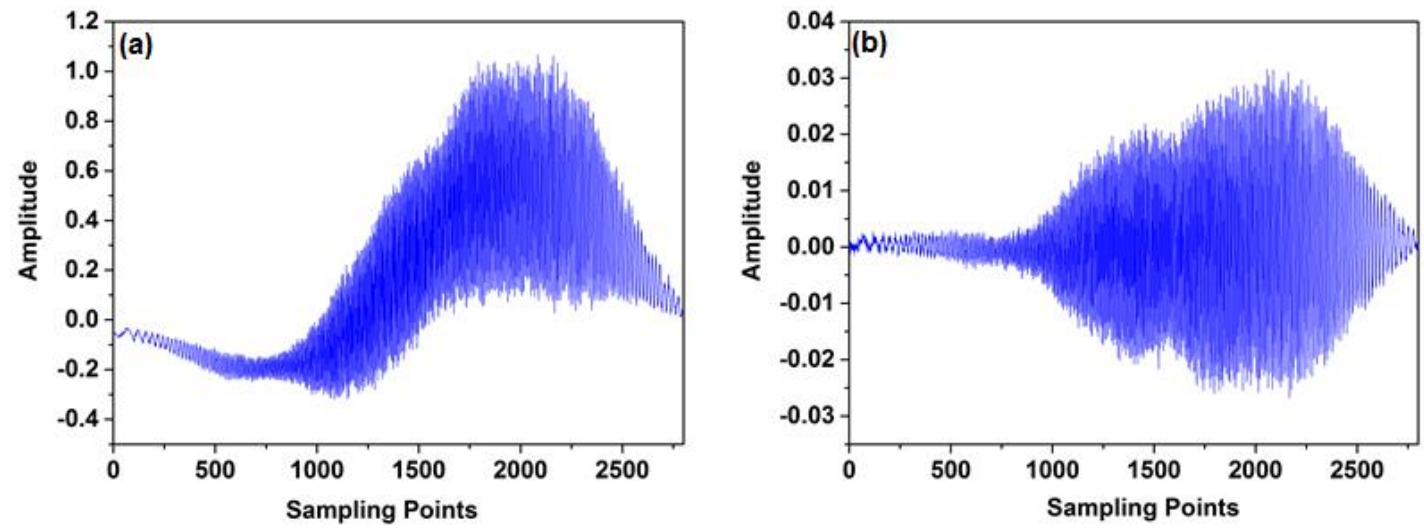

Figure 3.9 Spectral waveform (a) before background subtraction, and (b) after background subtraction.

\subsubsection{Spectral calibration and resampling}

The mechanical wavelength tuning in the frequency-swept laser sources cause the wavenumber to be swept nonlinearly and to vary within subsequent sweeps which are referred to as intra and inter-sweep variability, respectively. Fourier transformation on the non-linearly sampled OCT data will lead to the broadening of the PSF and deterioration of sensitivity. Therefore, the acquired OCT signal must be resampled into the uniform kspace intervals prior to Fourier transformation. Moreover, a real-time calibration is required to correct the inter-sweep variability. A spectral phase-based real-time automaticcalibration scheme is implemented in the developed SSOCT system. In this approach, fundamental component representing the surface reflection from the sample specimen is used as a calibration signal. This method avoids the use of auxiliary interferometer for calibration signal generation and additional channel for its acquisition. The unwrapped phase representing non-linear sweeping characteristics of the laser source is extracted from the reference signal. The phase-linearization (calibration) with improved accuracy is 
achieved by using a resampling function for calibration. The resampling function is obtained by normalization and rescaling of the obtained phase data. The fractional time index values corresponding to the linearized phase data are calculated from the resampling function. The OCT signal is resampled at these fractional time index values using a spline interpolation scheme. A detailed illustration of the concept and performance of the proposed automatic spectral phase-based calibration scheme is given in chapter 4. Figures 3.10 (a) and (b) show the spectral waveform representing a mirror reflection before and after calibration. Figures 3.10 (c) and (d) illustrate the impact of the calibration scheme on the PSF. In the absence of calibration, the PSF is largely spread and contains number of oscillations. This results in the degradation of the axial resolution. On the other hand, the proposed calibration scheme significantly improved the axial resolution, which is evident from the narrow PSF.
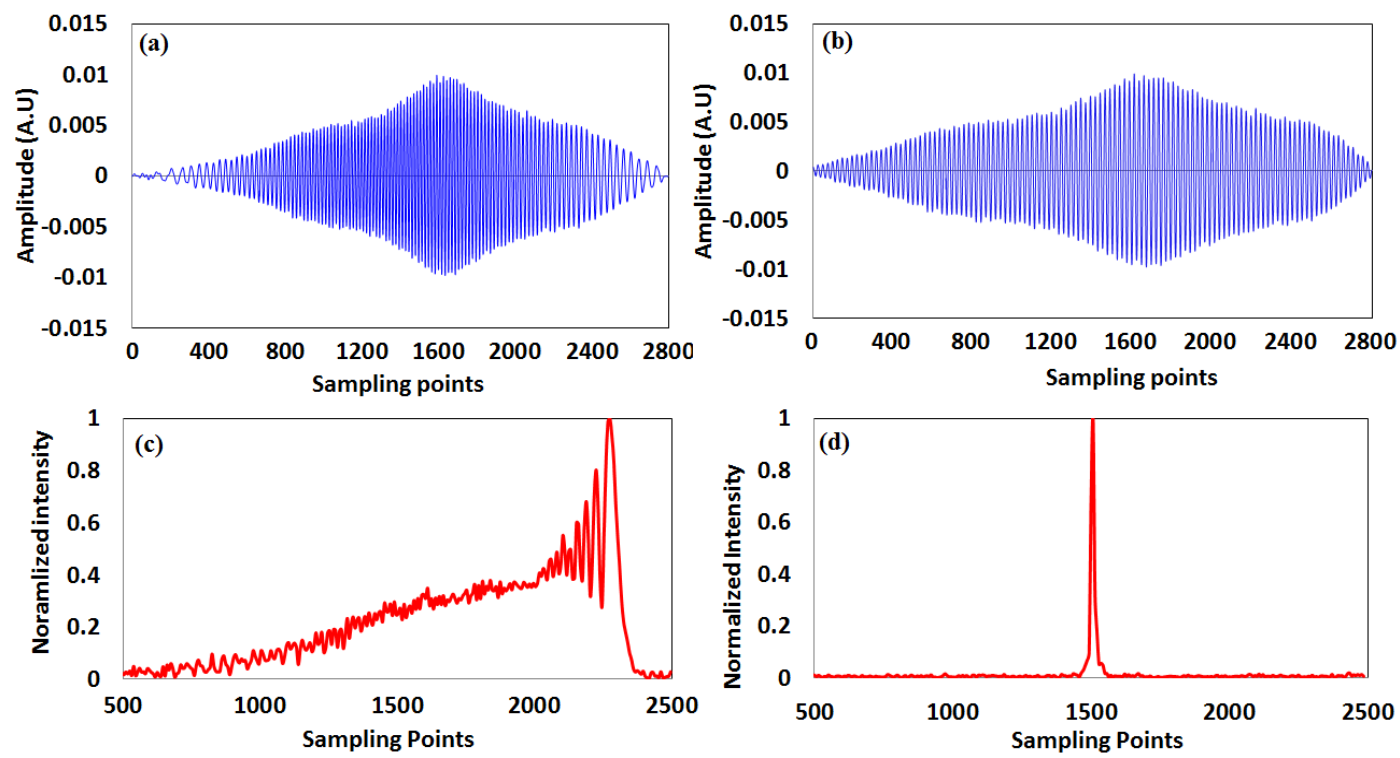

Figure 3.10 (a) and (b) shows spectral waveform and PSF before sampling and (b) and (d), after sampling. 


\subsubsection{Dispersion compensation}

Dispersion is a phenomenon by which the speed of the light in a medium depends on wavelength. If the total group indexes of all media in the sample and reference arms are mismatched, the axial resolution will be degraded.

A numerical algorithm for computationally correcting the second order dispersion is implemented in the developed system. The dispersion can be efficiently corrected by eliminating the dispersion-induced phase contribution from the spectral phase of the OCT signal. The spectral modulation term in the OCT signal can be expressed as:

$$
\cos \left(k(\omega) z+\varphi_{\text {disp }}(k(\omega))=\operatorname{Real}\left\{\exp \left[\mathrm{i}\left(\cos \left(k(\omega) z+\varphi_{\text {disp }}(k(\omega))\right)\right]\right\}\right.\right.
$$

Here $k(\omega)=\frac{\mathrm{n}(\omega) \omega}{c}$ is the wavenumber as a function of frequency $\omega$, and $\mathrm{z}$ is the optical path length difference. $\varphi_{\text {disp }}(k)$ represents the dispersion-induced phase contribution. Equation 3.8 shows that in the absence of dispersion, the spectral phase is directly proportional to optical path length difference $k(\omega) z$. Therefore, the spectral phase function must be linear for calibrated spectral fringes. The phase contribution due to the dispersion can be easily isolated by subtracting the instantaneous phase from the measured linear phase function. Finally, the dispersion is corrected by multiplying the fringe signal by complex conjugate of dispersion-induced phase.

The algorithm of the numerical dispersion compensation is given below.

Let $\varphi(k, i)$ represents the linear phase function of the spectral fringes obtained from a mirror reflection or specular reflection from the sample surface.

1. Calculate phase function $\varphi(k, i)$ 
2. Compute the linearly fitting coefficients $a$ (slope) and $b$ (Y intercept) using a robust linear regression.

3. Calculate the dispersion-induced phase: $\varphi_{\text {Disp }}(k)=\bar{\varphi}(k)-a k-b$

4. Multiply the fringe signal by $\exp \left(-i \varphi_{\text {Disp }}(k)\right)$

Figure 3.11 illustrates the impact of dispersion in OCT signal. Figure 3.11 (a) shows the resampled spectral waveform (blue) corresponding to a mirror reflection, which is not altered by dispersion. The static dispersion induced by the system if any, will be corrected by the spectral calibration scheme. The dispersion is introduced by inserting a thick glass plate in the optical path. The position of the reference is adjusted such that position of the PSF is unaltered. The spectral waveform shown in red color represents the resultant spectral waveform. The sample induces the dispersion and position of the waveform is shifted relative to waveform without dispersion. This relative shift in position is observed as the non-linear phase shift caused by the dispersion. The phase shift induced by the sample dispersion is more evident from the unwrapped phase plot given in Figures 3.11 (b) and 3.11 (d). Figure 3.11 (c) shows the PSF obtained before and after the dispersion correction. It is observed that PSF is broadened in the presence of the dispersion, leading to degradation of axial resolution. Figure 3.11 (c) further shows that FWHM of the PSF is reduced when the dispersion is corrected using proposed method. 

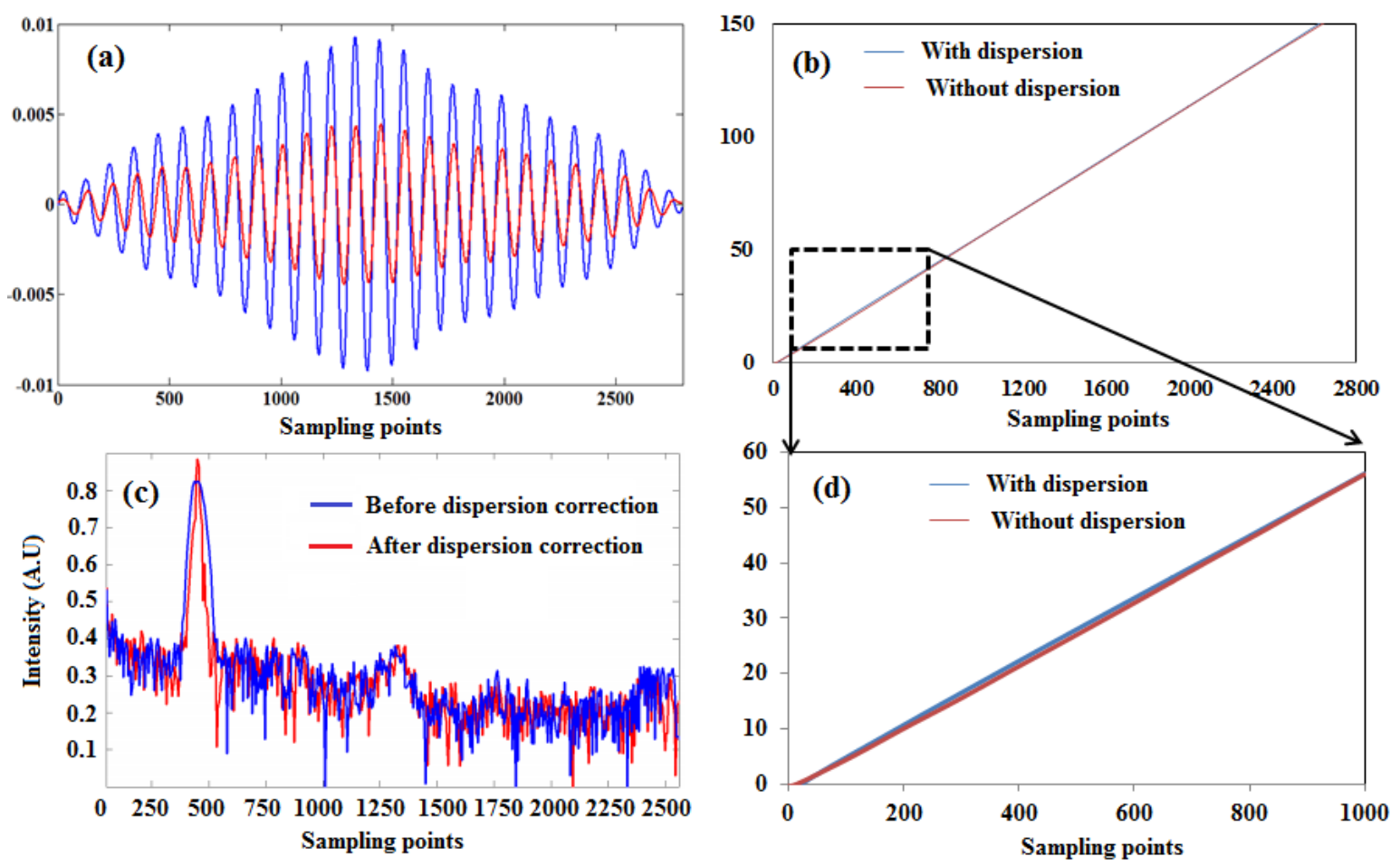

Figure 3.11 Illustration of dispersion (a) Spectral waveform with (red) and without dispersion (blue) (b) unwrapped phase plot (c) PSF before (blue) and after dispersion correction (red) (d) zoomed image of selected portion.

\subsubsection{Spectral apodization}

Figure 3.5 shows that the laser source used in the developed system exhibits non-Gaussian spectrum profile. This causes side lobes in the PSF. The presence of side lobes can blur the adjacent features and induce artifacts in the images. Spectral apodization is used to reshape the envelope of the interferogram so as to get the PSF with minimum side lobes and better contrast. Typically, windowing techniques are preferred for reshaping since they are faster and can be performed digitally. Figure 3.12 (a) and (b) demonstrate the transformation of the fringe shape after applying the Hamming window function. The expression of the hamming window is given by [149]

$$
w(n)=0.54-0.46 \cos \left(\frac{2 \pi n}{M}\right)
$$


Figure 3.12 (c) shows that the side lobes in the PSF is significantly suppressed after the apodization.
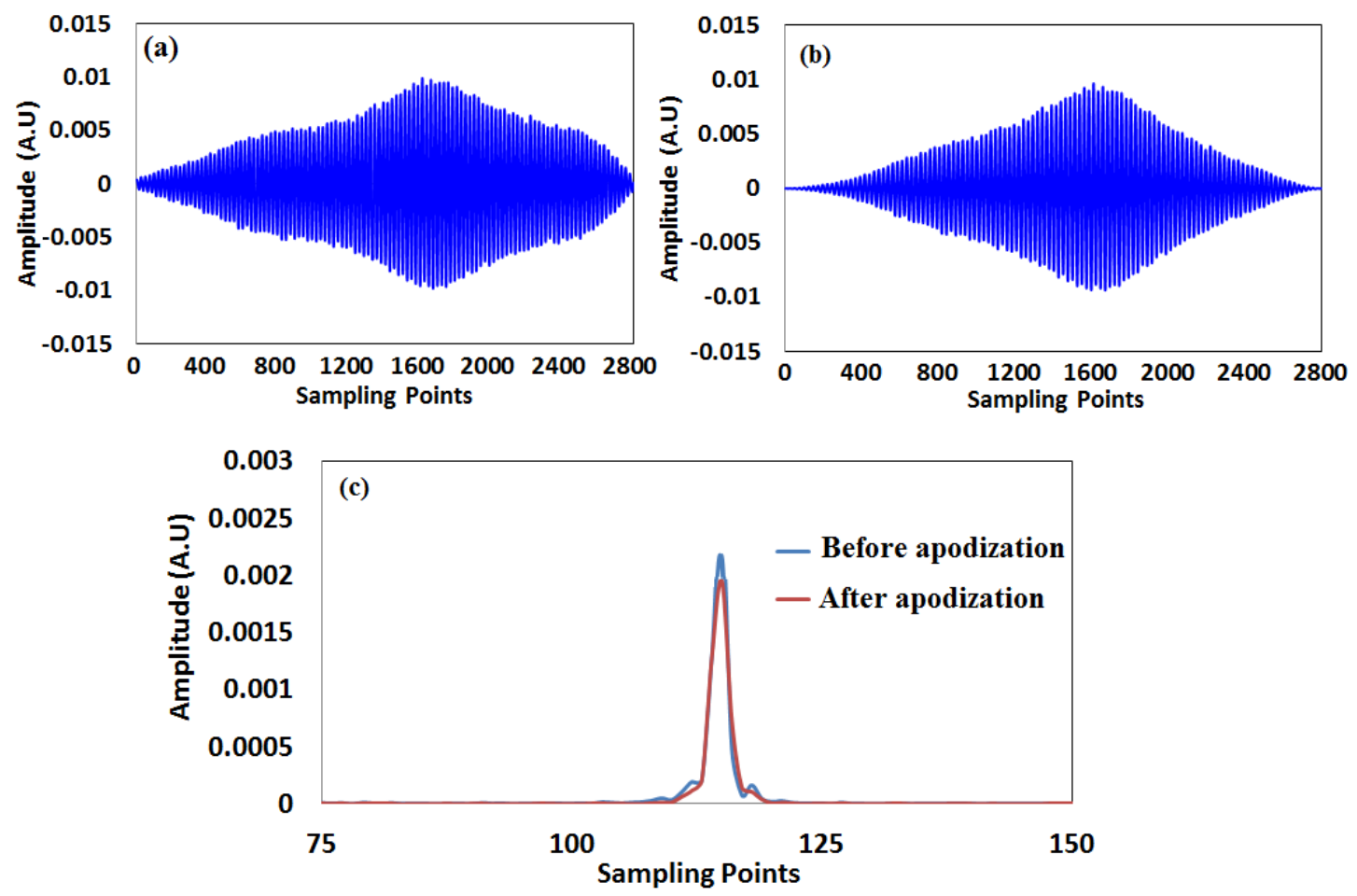

Figure 3.12 Spectral apodization using Hamming window (a) before apodization, and (b) after apodization. (c) PSF obtained before (blue) and after apodization (red).

\subsubsection{Fourier transform and image construction}

SSOCT system constructs images by performing inverse fast Fourier transform of the spectral interferogram that are linearly sampled in frequency space. Logarithmic nonlinearity is used to compress the processed data to fit the dynamic range for display and for brightness correction. The magnitude of the data is then compressed into 8-bit for gray scale image display where the maximum gray value assigned is 255 . 


\subsection{Results and discussion}

\subsubsection{Characterization of the proposed SSOCT system}

This section describes the experimental procedures and results for evaluating the spatial resolution, sensitivity and imaging depth of the proposed SSOCT system. The experimentally determined values of imaging parameters are compared with theoretical values.

3.5.1.1 Spatial resolutions: Unlike confocal microscopy, the mechanism that governs the axial and the transverse resolutions in OCT are decoupled. The transverse resolution of the OCT is determined by the size of the beam spot on the sample which depends on the numerical aperture of the focusing optics and the wavelength of the light source. Light in the sample arm is collimated and focused onto the sample surface by a commercially available microscope objective with 5X magnification (LSM03, Thorlabs Inc., USA), specially designed for OCT applications and optimized for infrared imaging at $1300 \mathrm{~nm}$.

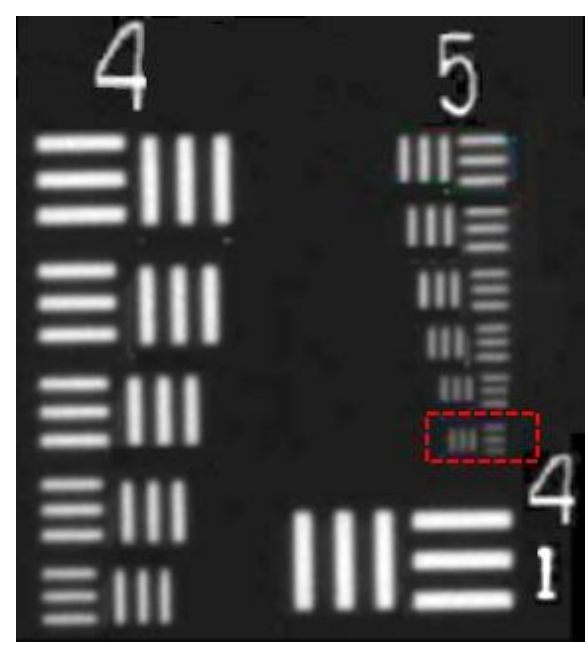

Figure 3.13 Enface image of the USAF chart demonstrating the lateral resolution of the imaging system. 
The focal length and working distance of the objective are $36 \mathrm{~mm}$ and $25 \mathrm{~mm}$, respectively. The lateral resolution of the imaging system is quantified using a USAF resolution chart. Figure 3.13 shows that the smallest grating that can be resolved by the imaging system has a period of $17.5 \mu \mathrm{m}$.

In OCT, the maximum achievable axial resolution is determined by the center wavelength $\lambda_{0}$ and bandwidth $\Delta \lambda$ of the broadband source. According to Equation (2.8), the theoretical axial resolution of the system with source parameters $\lambda_{0}=1320 \mathrm{~nm}$ and $\Delta \lambda=100 \mathrm{~nm}$ is $\sim 8$ $\mu \mathrm{m}$ in air $(\mathrm{n}=1)$. The axial resolution is experimentally measured as the full width at half maximum (FWHM) value of the PSF obtained. PSF is measured by computing the inverse Fourier transformation (IFFT) of the interference fringes obtained by placing a mirror at the sample arm. Figure 3.14, shows the Gaussian approximated PSF. The FWHM of the PSF is measured to be $8.3 \mu \mathrm{m}$. It has to be noted that the experimentally measured value closely agree with the theoretical value.

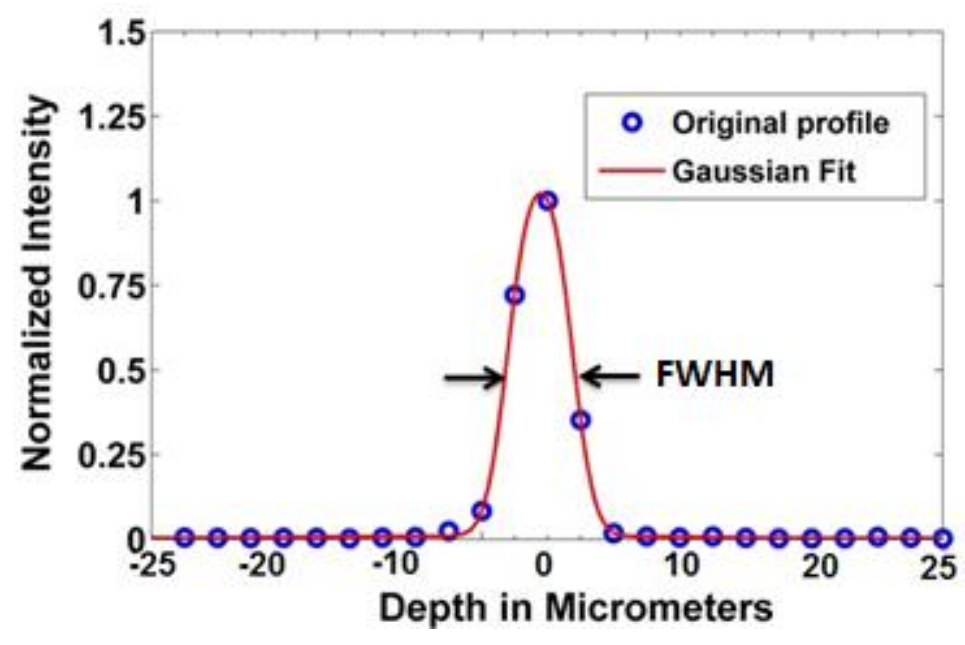

Figure 3.14 Gaussian approximated point spread function

3.5.1.2 Sensitivity: Sensitivity of an OCT system is defined as the ratio of the incident signal power on the sample to the minimum detectable power that is back reflected from a 
certain depth corresponding to the path difference $\Delta z$. The shot-noise limited sensitivity of a SSOCT system is given by [6]

$$
\Sigma_{\text {SSOCT }}=10 \log \left(\frac{\rho S \Delta t}{2 \mathrm{e}}\right)
$$

where $\rho$ is the responsivity of the detector, $\mathrm{S}$ is the source power, $\Delta \mathrm{t}$ is the sweep time and e is the electronic charge. Considering a sweep time of $62.5 \mu$ s and an incident power of $650 \mu \mathrm{W}$ on the sample, the theoretical sensitivity is calculated to be $\sim 111 \mathrm{~dB}$ based on Equation (3.10). Experimental sensitivity is quantified as 20 times the logarithmic ratio of the peak value of the PSF obtained to the standard deviation of the noise floor by blocking the sample arm. Since the direct measurement of the PSF may saturate the detector, a combination mirror and an attenuator are used at the sample arm for sensitivity measurement. The sensitivity of proposed system is characterized by placing a mirror and neutral density filter (ND) with optical density (OD) of 3 at the sample arm. Considering the double passage thorough ND filter, the combination of mirror and ND filter gives an attenuation of $-60 \mathrm{~dB}$. The sensitivity is then quantified by following equation

$$
\Sigma_{d B}=20 \log \left(\frac{\left(i_{D}(\Delta \mathrm{z})\right)}{\sigma(\Delta \mathrm{z})}\right)+20.0 D
$$

where "." represents multiplication, $i_{D}(\Delta z)$ is the detector current corresponding to the $\mathrm{PSF}$, and $\sigma(\Delta z)$ represents the standard deviation of the noise floor.

A SNR of $50 \mathrm{~dB}$ was measured near to the zero optical path length. This shows a peak sensitivity of $110 \mathrm{~dB}$ based on the Equation (3.11). 
Figure 3.15 (a) shows that PSF acquired from different depth positions. As discussed in section 3.1, the amplitude of the SSOCT system decreases with the imaging depth. Figure 3.15 (b) shows the sensitivity-roll off plot obtained for the developed SSOCT system.
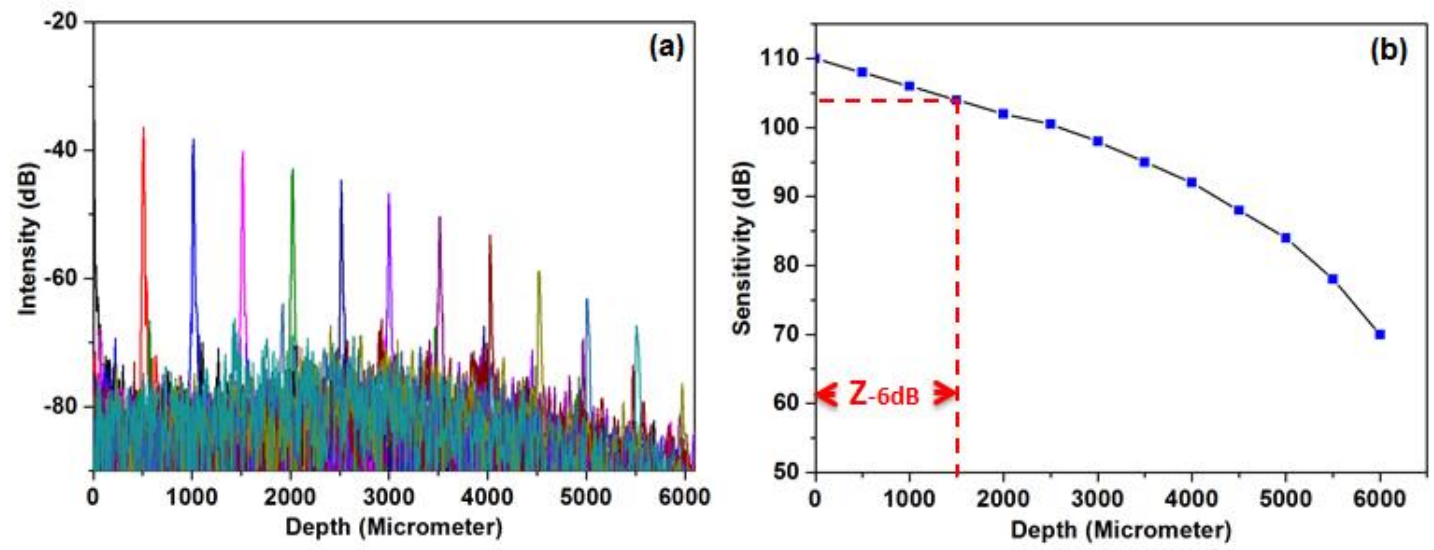

Figure 3.15 (a) PSF at different depth positions, and (b) sensitivity fall-off as function of depth

3.5.1.3 Depth range: The maximum imaging range $\left(Z_{\max }\right)$ in OCT is determined by the central wavelength of the swept source $\left(\lambda_{0}\right)$ and the spectral sampling interval $\left(\delta_{\mathrm{s}} \lambda\right)$. Considering the parameter values $\lambda_{0}=1320 \mathrm{~nm}$ and $\delta_{\mathrm{s}} \lambda=0.07$, the maximum imaging range is calculated to be $6 \mathrm{~mm}$ using Equation (3.6). This value is found to be in good agreement with the experimentally determined value from Figure 3.15 (a). The principal imaging range $\left(Z_{-6 d B}\right)$ is limited by the instantaneous coherence length of the laser. Considering the swept-source parameters $\lambda_{0}=1320 \mathrm{~nm}$ and $\delta_{\mathrm{r}} \lambda=0.290 \mathrm{~nm}$, Equation (3.5) gives a theoretical value of $1.5 \mathrm{~mm}$. Further, the $-6 \mathrm{~dB}$ imaging range is experimentally determined from the sensitivity-roll off curve. Figure 3.15 (a) shows that the depth corresponding to $-6 \mathrm{~dB}$ is $1.5 \mathrm{~mm}$. This shows that the experimentally measured value is consistent with the theoretically determined value. 


\subsubsection{Experimental validation}

The enhanced imaging capability of SSOCT system and its feasibility for in-vivo imaging are demonstrated by acquiring cross-sectional images of different biological samples. Imaging is performed at a rate of 22 frames per second. The obtained images have pixels size of $512 \times 1024$. The performance of the system in highly scattering samples is analyzed by acquiring the cross-sectional image of a human finger nail-skin region in-vivo. Figure 3.16(a) shows the cross-sectional image of a finger nail-skin region. The obtained image with high contrast and resolution clearly shows different subsurface features such as the nail plate (NP), nail bed (NB), demis (D), Epidermis (ED) and blood vessels. The performance of the system is further analyzed by imaging the iridocorneal angle of a pig eye. The ocular medium represents a highly absorptive and dispersive sample. In this case, OCT signal will be severely degraded due to the attenuation and distortion. The attenuation is mainly caused by the wavelength dependent absorption of the ocular medium (aqueous humor), whereas the distortion is contributed by the chromatic dispersion. Figure 3.16(b) shows the cross-sectional image of the iridocorneal angle. The high sensitivity of the developed system allows detection of weaker signal from the tissue layers beneath the aqueous humor. Similarly, dispersion compensation scheme implemented in the system efficiently corrects the distortion caused by the medium. Consequently, a high quality image was obtained, in which the clinically features such as Iris (IS), Cornea (CA), sclera (SA), and trabecular mesh (TM) region were clearly observed. 

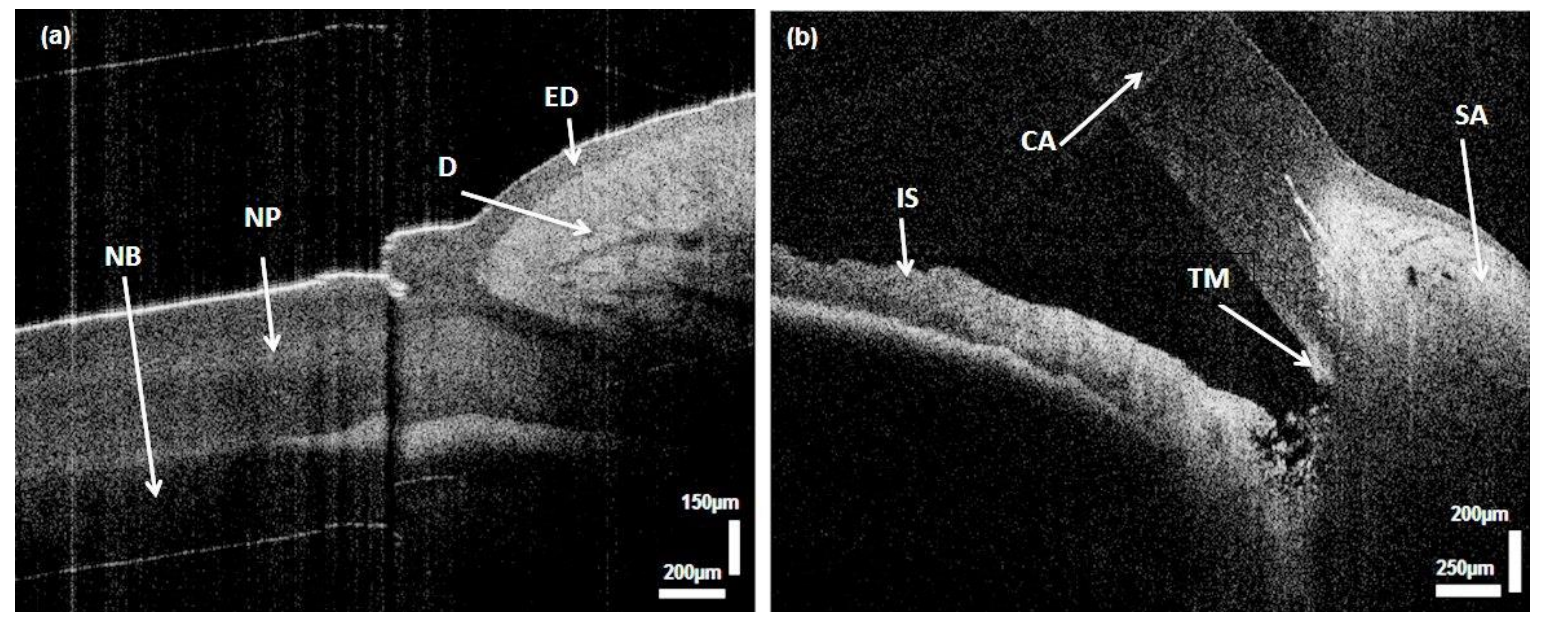

Figure 3.16 Images acquired by SSOCT system (a) finger nail-skin region (b) iridocorneal angle of pig eye. (Images are resized for better visual representation).

\subsection{Conclusion}

In summary, a simple and compact swept source based optical coherence tomography system with improved imaging capability is demonstrated. The non-reflective reference arm based interferometer provides a highly stable and compact configuration for SSOCT system. The implementation of automatic calibration scheme further simplifies system design by avoiding the external hardware resources for calibration. The comprehensive analysis of hardware and software configurations for the proposed system is systematically presented by discussing relevant theoretical and technical aspects. The spectral fringes generated by the interferometer are detected by the dual balanced detection scheme followed by digital sampling using a high speed digitizer. Balanced detection followed by software based background subtraction allows the efficient removal of DC components and other artifacts in the OCT signal. Enhancement of axial resolution and sensitivity are achieved by novel spectral phase-based automatic calibration scheme and dispersion compensation, respectively. It is worth to mention that that experimentally measured values of the imaging parameters such as axial resolution, sensitivity and imaging depth 
are in good agreement with the theoretically computed values. This demonstrates the efficiency of the proposed hardware and software schemes for achieving accurate and precise measurements. High resolution and high contrast images of a finger nail-skin region and an iridocorneal angle show the applicability of the proposed system for in-vivo analysis of complex biological systems.

Next chapter details the implementation of a real-time automatic calibration scheme for SSOCT system. Details including the theoretical concept, implementation and performance analysis will be given. 


\section{Chapter 4: Real-time automatic spectral calibration for SSOCT systems}

In this chapter, a real-time automatic calibration for SSOCT system is presented. This chapter begins with the physics of phase-based calibration method and details the relevant fundamental concepts. Implementation details of the proposed calibration method and signal processing routines will be described. This is followed by the illustration of the performance using a mirror and a tissue sample. The performance of the proposed method is quantitatively evaluated in terms of major imaging parameters such as axial resolution, sensitivity roll-off and imaging depth, and compared with existing schemes.

\subsection{Introduction}

SSOCT system is based on spectral interferometry in which a near-monochromatic light is swept/tuned over a broad bandwidth and the spectral interferograms are acquired sequentially in time space. This means that the obtained spectral interferogram is a function of time, but not a function of wave number $k$. Typically in Fourier domain OCT systems, depth information is retrieved by performing inverse fast Fourier transform of the spectral interferogram in uniform $k$-space. The uncertainty associated with laser generation and the mechanical wavelength tuning of the laser source causes the wavenumber to be swept nonlinearly. Therefore, the spectral interferogram acquired in time space cannot be directly mapped to $k$ space. The inverse fast Fourier transform on the non-uniformly sampled OCT signal in $k$-space will deteriorate the sensitivity and axial resolution, leading to a poor image quality. In order to circumvent this issue, the acquired OCT signal must be 
resampled at equidistant $k$-space intervals prior to performing Fourier transform. In contrast to the spectrometer based systems, the non-linear $k$-space distribution of the SSOCT system is not known a priori. If the swept laser source can maintain highly stable and repeatable inter-scans, frequency-time characteristics once derived can be repeatedly used for calibration. However, in most of the swept laser sources used in SSOCT, especially with fast scanning rate, the stability and repeatability of frequency scanning is always compromised. Therefore, a real-time calibration is always preferred in SSOCT systems to accomplish highly precise and stable measurement throughout the duration of imaging.

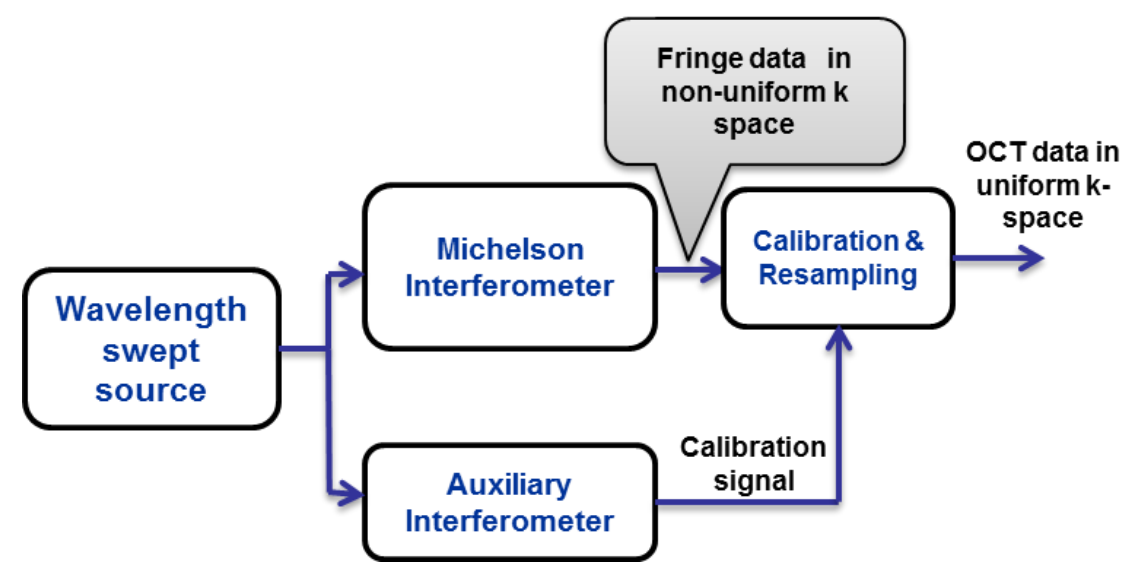

Figure 4.1 Block schematic of typical calibration setup in SSOCT systems

The most common methodology used for calibration in SSOCT systems is depicted in Figure 4.1. In this approach, an auxiliary interferometer is used to generate the calibration signal and an additional data channel to record it. This method allows digital manipulation of the calibration signals for the implementation of different calibration schemes based on digital clocking, intensity, and phase-based algorithms. The use of additional hardware resources makes the system highly complex and expensive. The accuracy and processing 
speed of a calibration scheme are significantly influenced by the algorithm used for calibration. The spectral phase-based algorithms are highly promising for accurate calibration due to the linear-relationship between the spectral phase and wavenumber of interferogram. However, the functional approximation such as polynomial fitting adopted in the spectral phase-based algorithms causes errors in computation and limits the accuracy of calibration. Higher accuracy can be achieved by fitting polynomials with higher orders. However, it would make the computation intensive and limits their applicability in realtime calibration.

This chapter presents a highly accurate and cost-effective solution for real-time calibration in SSOCT systems. The calibration is performed by an automatic method, that doesn't require additional interferometer for calibration. A novel spectral-phase based algorithm based on the absolute phase function of the calibration signal is proposed and demonstrated in this chapter. The following sections detail the proposed method for calibration and its implementation in the developed SSOCT system.

\subsection{Implementation of proposed automatic calibration scheme}

The proposed automatic calibration is performed in two stages. First stage represents the generation of reference signal for calibration. Typically in FDOCT systems, signals coming from different depths result in different frequency components in the spectral interferogram. However, the surface reflection of the sample generates a large spectral modulation due to a high refractive index discontinuity. This spectral modulation essentially determines the fundamental frequency of the interference fringe. This frequency component can be separated using a narrow band-pass filter and used as the calibration signal. The filter with higher-order transfer function and a high quality factor allows the 
efficient rejection of noise and detection of fundamental frequency component of the composite signal. The major advantage of this approach is that the calibration can be performed during the scanning operation and does not require additional hardware resources for calibration.

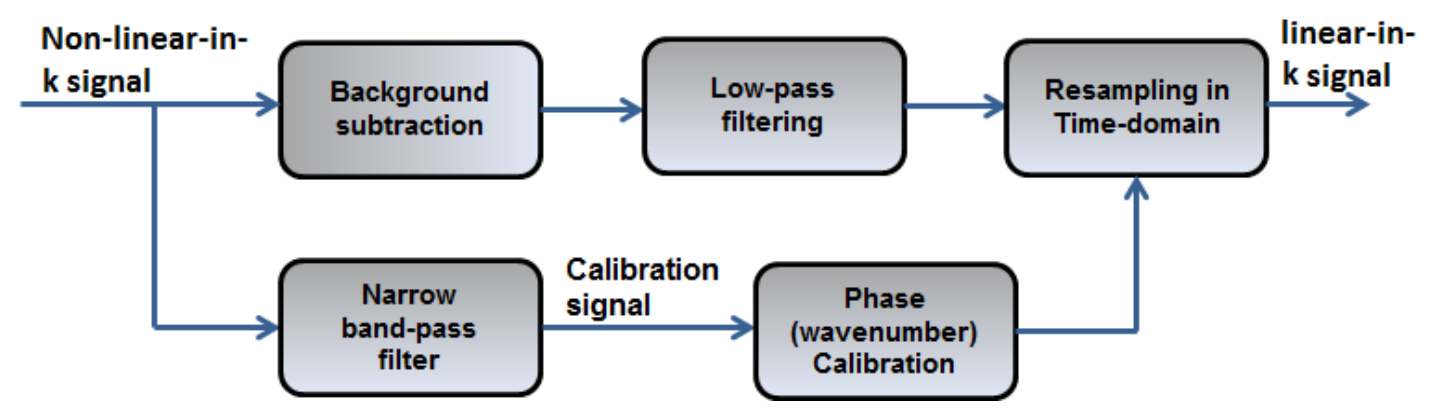

Figure 4.2 Block schematic of the proposed real time automatic calibration scheme

In the second stage, wavenumber linearization of the calibration signal was performed using a spectral phase-based calibration scheme. In this approach, the spectral phase function representing the non-linear sweeping characteristic of the laser source was extracted from the calibration signal. The obtained phase function was normalized and rescaled into maximum number of sampling points. The resampling function was obtained by swapping the axes of the rescaled phase function. Accordingly, the $\mathrm{x}$-axis of the resampling function represents the equidistantly calibrated phase values and $\mathrm{y}$-axis represents the corresponding time index values. The fractional time index values corresponding to the linearized phase data were directly estimated from the resampling function and used to resample OCT signals in time-domain. Unlike the existing timedomain schemes, proposed method does not fit higher order polynomial to phase data for the calculation of time-index values. This improves the accuracy of calibration, 
irrespective of the non-linearity of wavelength sweeping. Finally, the acquired OCT signals were resampled at these fractional time index values using spline interpolation.

In order to accomplish a precise and stable measurement, real-time calibration was performed on individual spectral interferograms based on their respective sweeping characteristics (wavenumber- time characteristics). Figure 4.2 represents the block schematic of the proposed real-time automatic calibration scheme. The proposed method extracts the calibration signal from every spectral interferogram (Non-linear-in-k signal) using an adaptive narrow bandpass filter. The adaptive filter allows the separation of fundamental component irrespective of the location of surface reflection. The resampling function corresponding to individual spectral interferograms was constructed by the spectral phase-based approach. The background subtracted and noise removed (low-pass filtered) OCT signals were resampled directly in time domain using their respective resampling function. Thus the implemented parallel extraction of the calibration signal and phase linearization which is simple and fast enables real-time calibration of OCT signals.

\subsubsection{Illustration using mirror reflection}

The proposed method is illustrated by acquiring the fringe signal corresponding to the specular reflection from a mirror. The interference signal from a Michelson interferometer sampled in uniform time space by the digitizer, giving $\mathrm{N}$-point discrete time-domain signal $I_{D}\left(t_{i}\right)$ can be expressed as

$$
I_{D}\left(t_{i}\right)=S\left(t_{i}\right) \cdot R \cdot \cos \left(k\left[t_{i}\right] \cdot d+\varphi_{D i s p}\right) \quad i=1,2,3, \ldots, N
$$

where $R$ represents a scale factor, $d$ is optical path length difference between the two arms

of the interferometer, $t_{i}$ is the time index of the $i^{\text {th }}$ sample, and $S\left(t_{i}\right)$ is the spectral 
envelope of the swept source. It can be seen that the spectral phase is directly proportional to the wavenumber $(k)$. The term $\varphi_{\text {Disp }}$ represents the dispersion induced phase distortion caused by the sample. This term is neglected in the subsequent analysis since the fringe signal corresponding to the air-sample interface is only considered. This leads to the assumption that the phase non-linearity of the signal is solely determined by the frequency sweeping characteristics of the laser.
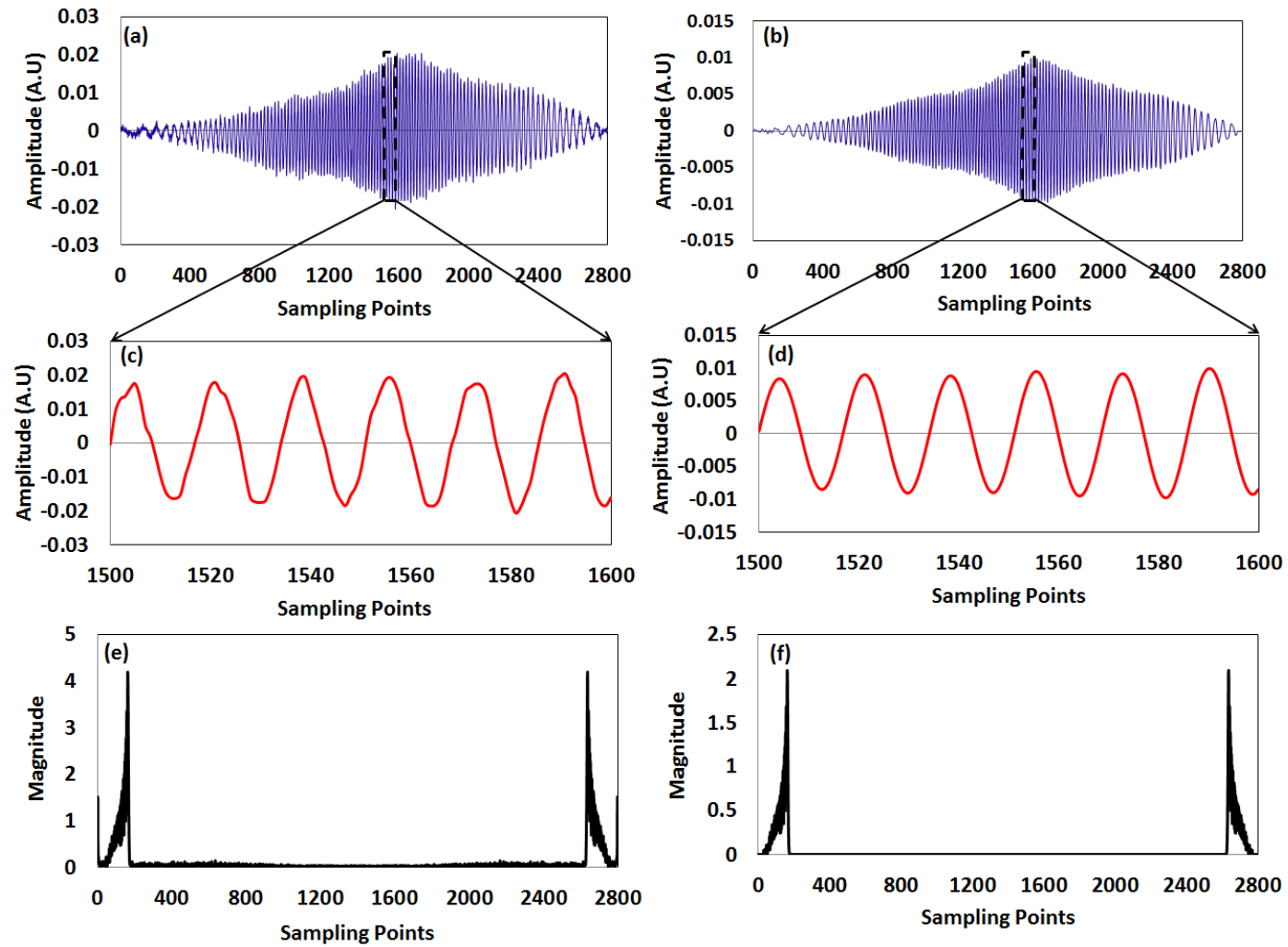

Figure 4.3 (a) Fringe signal before noise removal (b) fringe signal after noise removal (c) and (d) zoomed view of raw fringe signal before and after noise removal (e) and (f) FFT of fringe signal before and after noise removal.

Figure 4.3(a) represents the spectral waveform corresponding to the specular reflection from a mirror. The high frequency noise components present in the signal are removed by filtering as shown in Figures 4.3(b) and 4.3(d). Figure 4.3(b), also shows that spectral 
waveform is chirped, in which the periodicity of the spectral waveform is not constant. This is due to the non-linear sampling of spectral waveform in $k$-space. If left uncorrected, the chirping effect in the spectral waveform results in a broadened signal in time-domain as shown in Figure 4.3(f).

The proposed method makes use of unwrapped phase function of the reference signal for calibration. The unwrapped phase of the spectral waveform extracted via Hilbert transform is shown in Figure 4.4(a). The curved phase function shows that the spectral waveform is non-linearly distributed in $k$-space. The proposed method makes use of a resampling function for the calibration of OCT signals. The phase values are normalized and multiplied with the maximum number of sampling points using the relation $\left\{k_{1}, k_{2}, k_{3} \ldots k_{M}\right\} N / k_{M}$ in order to get the $N$ equidistant k-values. The resampling function is formed by swapping the axes of the unwrapped phase plot as shown in Figure 4.4(b). $\left\{k_{1}, k_{2}, k_{3} \ldots k_{M}\right\}$ represents the set of $k$-values (unwrapped phase) and $k_{M}$ corresponds to the maximum value of the phase obtained.
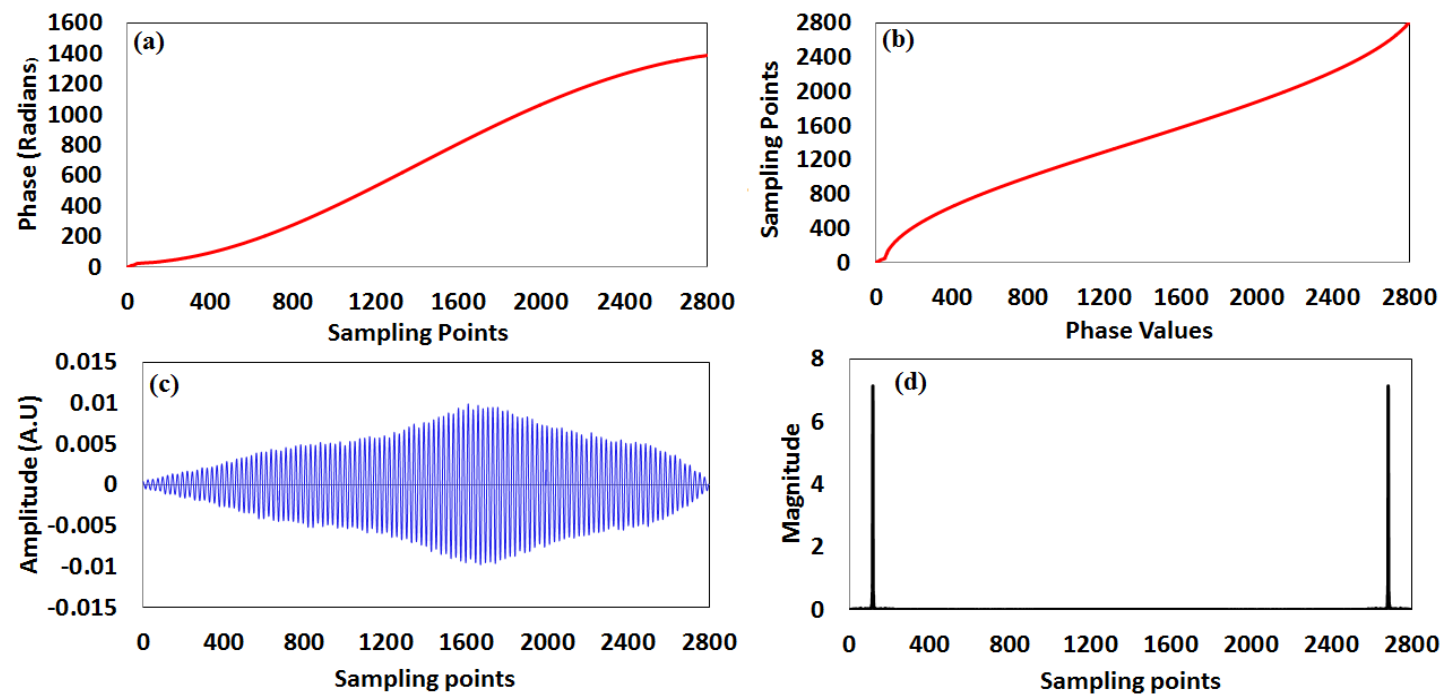

Figure 4. 4 (a) Unwrapped phase function (b) resampling function (c) resampled spectral waveform (d) Fourier transform of the resampled spectral waveform data. 
The non-linear fractional-time index values corresponding to the uniformly distributed phase values are computed directly from the resampling function. The acquired OCT traces are resampled at these fractional-time index values using a spline interpolation in order to get the k-linearized OCT data. Figures 4.4(c) and 4.4(d) represent the resampled signal and its Fourier transform in $k$-space, respectively. The line shaped spectrum illustrates that the fringes are spaced uniformly in $k$ space.
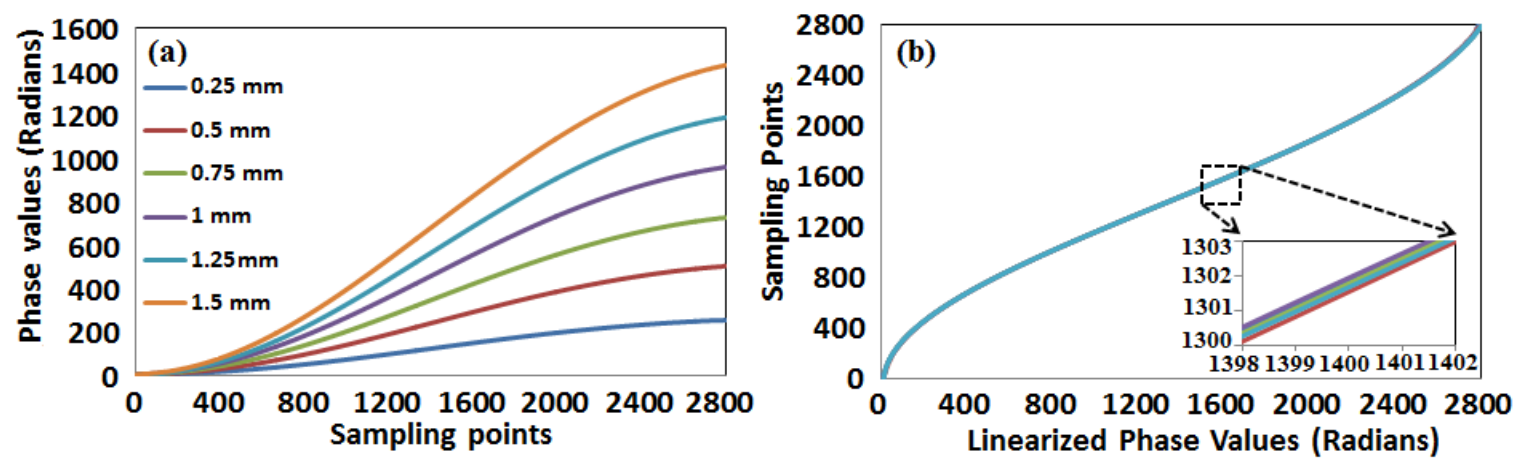

Figure 4.5 (a) Phase functions acquired from different depths, and (b) Resampling functions corresponding to the individual phase functions.

The potential of spectral phase-based approach for real-time automatic calibration is further investigated by acquiring phase functions from different depths. By placing a mirror in the sample arm, the optical path difference between the sample and reference arms was adjusted such that interferogram from different depths are recorded. Figure 4.5(a) represents unwrapped phase functions extracted from the spectral interferogram acquired from different depths. Figure 4.5(b) represents the resampling functions obtained by normalization and rescaling of the individual phase functions given in Figure 4.5(a). Figure 4.5(b) shows that spectral phase-based linearization scheme allows the efficient construction of resampling function regardless of the position of specular reflection. This aspect is quite important when the arbitrary reflection from a non-planar surface is considered. Figure 4.5(b) shows that the resampling functions do not superpose one over 
the other due to the inter-sweep variability (sweep to sweep variation) of the frequency swept laser source.

\subsubsection{Illustration using a sample tissue}

The feasibility of the proposed method for a practical imaging scenario is demonstrated by considering the Fresnel reflection from an air/tissue interface. Figure 4.6(a) represents the OCT image of a finger nail-skin region, in the absence of calibration. The spectral interferogram obtained from an arbitrary position of the sample is shown in figure 4.6(b).
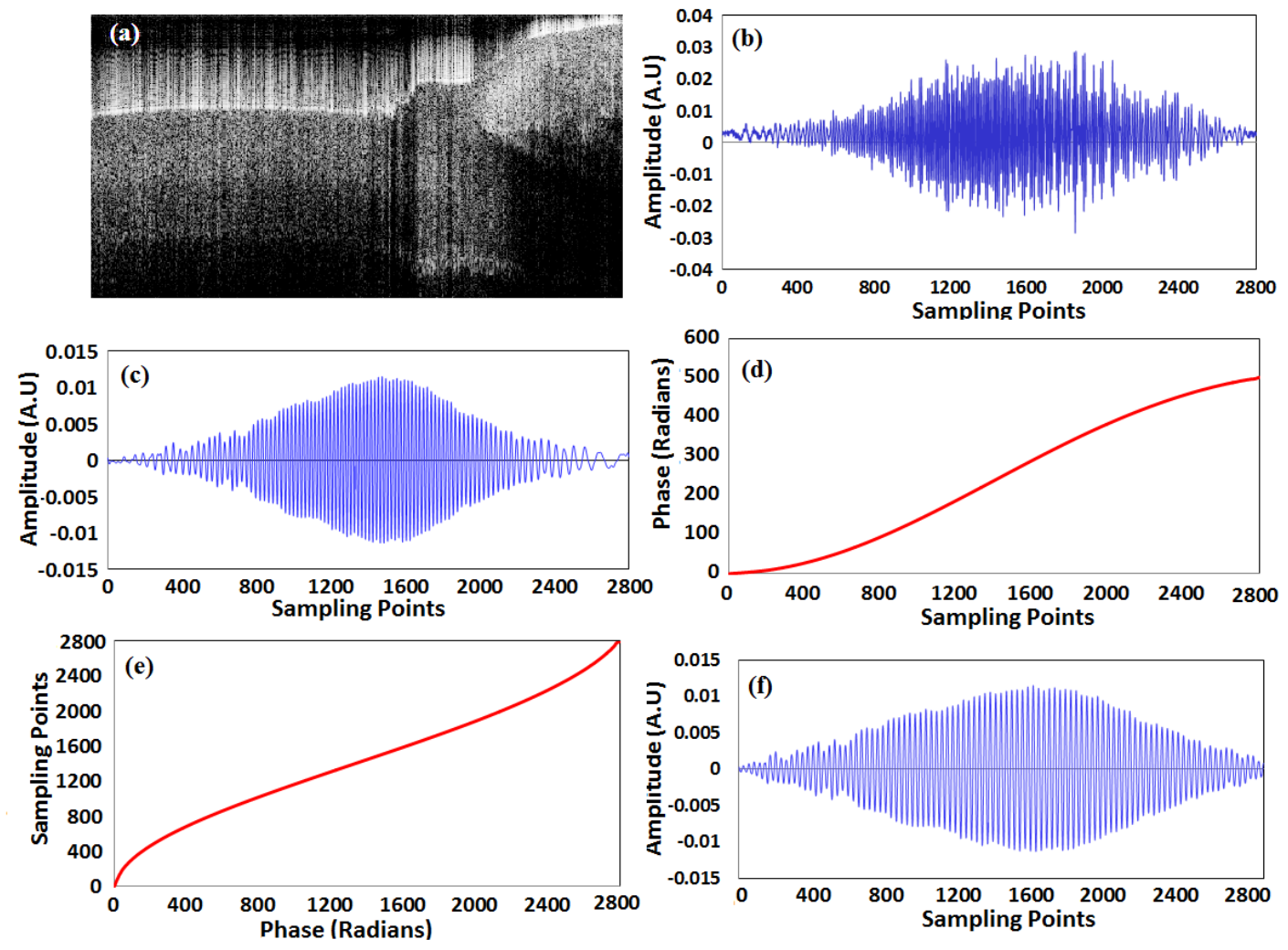

Figure 4.6 (a) Cross-sectional image of the finger nail-skin sample before calibration (b) A-scan acquired from an arbitrary position (c) Band-pass filtered signal representing the surface reflection (d) (d) Spectral phase function (e) Resampling function (f) Resampled signal.

This signal contains different spectral components representing the reflections from different subsurface layers with phase modulations induced by the medium. Figure 4.6(c) 
represents a fundamental frequency component representing the tissue surface reflection that is separated using narrow band-pass filter. This signal is used as the calibration signal for acquired spectral interferogram. Figures 4.6(d) and 4.6(e) represent the spectral phase function and resampling function, respectively. Figure 4.6(f) represents the resampled spectral signal.

\subsection{Performance analysis and discussion}

The performance of the proposed calibration scheme is quantitatively evaluated in terms of axial resolution, sensitivity roll-off, processing time and quality of the acquired images. Furthermore, the performance is compared with that of the existing phase-based time domain interpolation scheme with the polynomial fitting approach (PTDI) and the intensity-based scheme that rely on a fast nearest neighbor check algorithm (NNC) [23, 25]. The polynomial fitting-based time domain interpolation scheme is accomplished by extracting the unwrapped phase values of the calibration signal and fitting by a higher order polynomial function. The fitted higher order polynomial with the phase (wavenumber) as an independent variable and time as a dependent variable can be expressed as the following equation.

$$
t(k)=a+b k+c k^{2}+d k^{3}+e k^{4}+f k^{5}+g k^{6}+\ldots
$$

For a $6^{\text {th }}$ order polynomial fitting on the phase function gives the calibration coefficients a $=0.000364, \quad b=-0.2330, \quad c=0.000815, d=-3.34 * 10^{-7}, \quad e=6.93 * 10^{-11}, \quad f=-1.01 * 10^{-11}$ and $\mathrm{g}=6.17 * 10^{-19}$. Equidistantly spaced k-values are further computed by Equation (4.3). The fractional time index values corresponding to the equidistantly spaced k-values are calculated using the fitted polynomial relation [110] 


$$
k_{i}=k_{0}+\frac{k_{N-1}-k_{0}}{N-1} i
$$

Where $i=0,1,2 \ldots N-1$ represents the sampling instants. The acquired OCT traces are interpolated at these fractional index values using the spline interpolation.

In the case of intensity-based calibration scheme, time index values corresponding to the peak and valley points of the calibration signal are identified using a fast nearest neighbor check algorithm (NNC). Further, the sampling points are increased to 840 by adding time instances corresponding to zero crossing points. The numbers of points are further increased to 2800 points by zero padding so as to make it comparable with the phase-based schemes.

\subsubsection{Axial resolution}

The performance of the proposed scheme is evaluated by measuring the FWHM of the PSF, which determines the axial resolution of the system. Figure 4.7(a) shows the PSF obtained for different optical path differences (imaging depth) by placing a mirror at the sample arm followed by performing inverse FFT of the acquired fringes at each position. Figures 4.7(a) and 4.7(c) demonstrate that the proposed calibration scheme improves the axial resolution significantly which can be observed from the reduced FWHM of the PSF obtained. In addition, the FWHM obtained by the proposed calibration scheme is less than that obtained by PTDI with sixth-order polynomial fitting and the NNC scheme as demonstrated in Figure 4.7(d). The proposed calibration scheme gives a FHWM of $8.3 \mu \mathrm{m}$ for a PSF measured at a depth of $1.5 \mathrm{~mm}$ in air, whereas the reduced values of 9.3 and 9.9 $\mu \mathrm{m}$ are observed when PTDI (sixth-order) and NNC schemes are used. Furthermore, the axial resolution obtained by the proposed scheme is close to the theoretical value of $\sim 8$ 
$\mu \mathrm{m}$, which shows the improved accuracy of the calibration. Variations of the FWHM with the imaging depth for different schemes were also investigated, as shown in figure 4.7(b). From Figure 4.7(b), it is obvious that the proposed scheme keeps the axial resolution almost constant throughout the imaging depth, whereas the PTDI with higher order polynomial fitting and NNC schemes show the reduced axial resolution with the increase in depth. The axial resolution is found to be improved when tenth and fifteenth-order polynomial fittings are used; however, it is lower than the proposed scheme.
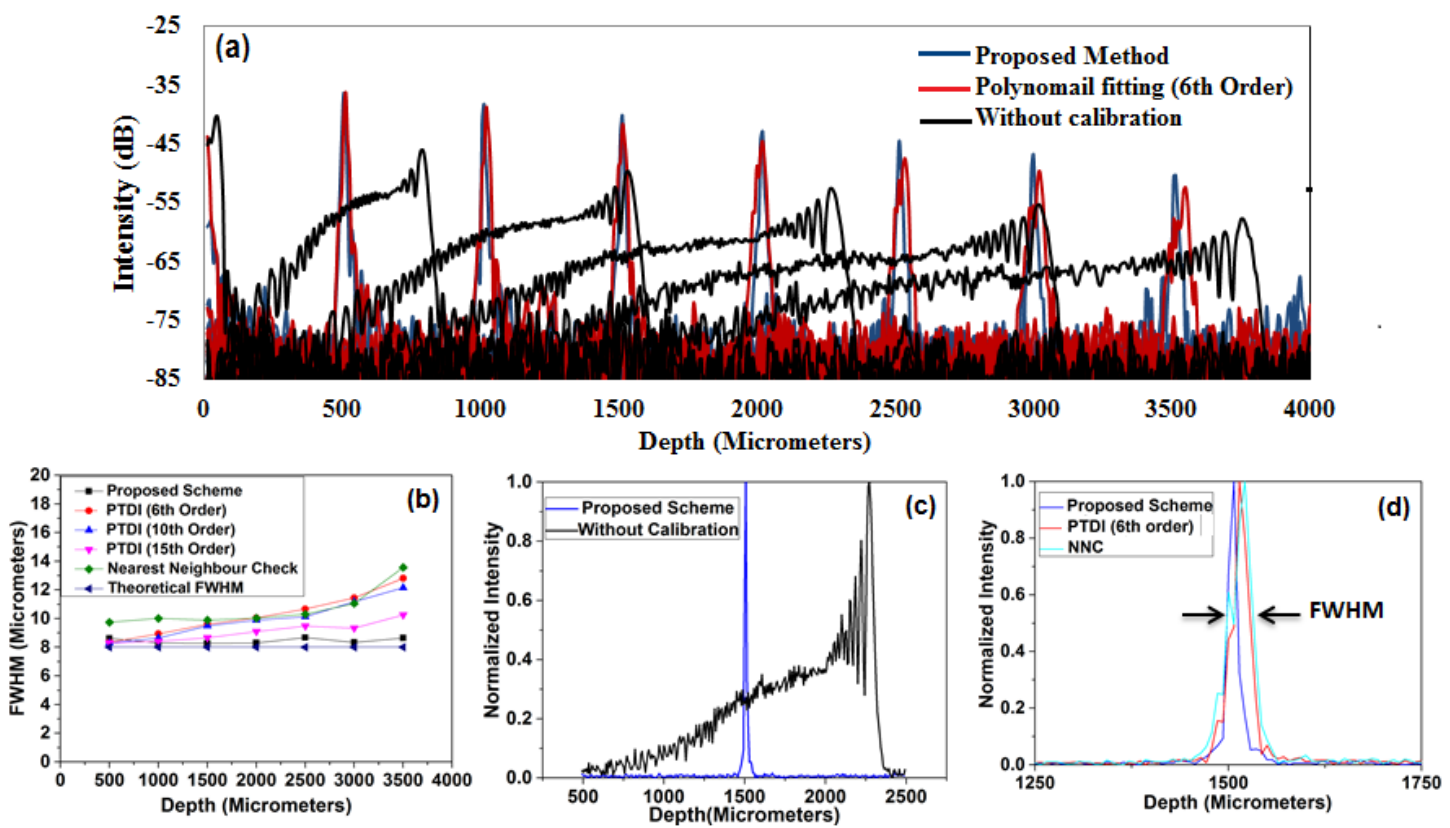

Figure 4.7 (a) PSF for the proposed scheme and PTDI with $6^{\text {th }}$ order polynomial fitting (b) FWHM at different depths (c) PSF for proposed scheme and without calibration scheme at $1.5 \mathrm{~mm}$ (d) PSF obtained for proposed scheme, PTDI with $6^{\text {th }}$ order and NNC scheme at $1.5 \mathrm{~mm}$.

\subsubsection{Sensitivity roll-off measurement}

The performance of the system under the proposed calibration scheme is also investigated by measuring the sensitivity roll-off with imaging depth. The sensitivity in $\mathrm{dB}$ is measured as 20 times the decadic logarithm of the ratio between the peak of the PSF and the standard deviation of the noise floor, which was measured by blocking the sample arm. The 
sensitivity and its roll-off for the proposed PTDI scheme with different polynomial order fittings and NNC are demonstrated in Figure 4.8. It is evident that the proposed calibration scheme gives better sensitivity and reduced roll-off compared to the other schemes. Furthermore, the imaging depth corresponding to the $6 \mathrm{~dB}$ sensitivity drop is measured as $1.5 \mathrm{~mm}$ for the proposed scheme and is in good agreement with the theoretical value. Reduced depths of 1.25 and $1.15 \mathrm{~mm}$ were obtained in the case of PTDI with sixth-order polynomial fitting and the NNC approach. However, a reduced fall-off was observed for PTDI with tenth and fifteenth-order fitting-still poorer than the proposed scheme.
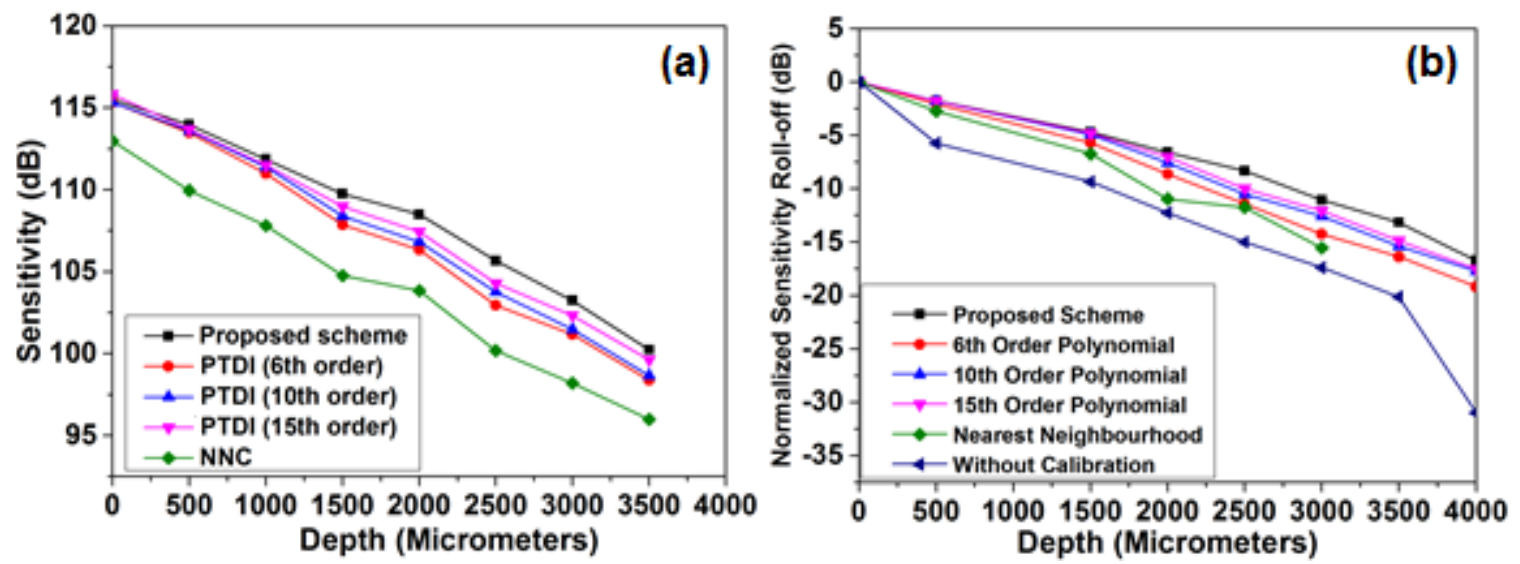

Figure 4.8 Sensitivity roll-off measurement of different calibration schemes (a) absolute values (b) normalized sensitivity roll-off curve.

\subsubsection{Computation time}

The processing time taken for the different calibration schemes was investigated using MATLAB script in post-processing mode. Average times of 5.8, 6.8 and $8.6 \mathrm{~ms}$ were obtained for the proposed scheme, NNC and PTDI with sixth-order fitting, respectively. It is evident that the processing time for the proposed scheme is less compared to PTDI and NNC scheme. Furthermore, an increased processing time of 13.4 and $17.8 \mathrm{~ms}$, were obtained for PTDI with tenth and fifteenth-order polynomial fitting, respectively. 


\subsubsection{Images acquired with different calibration schemes}

The performance of the proposed automatic calibration scheme was evaluated by acquiring cross-sectional images of the samples showing different reflectivity and surface geometry. Figure 4.9 illustrates the impact of the proposed calibration and resampling scheme in the quality of the image. The images of the finger nail and its adjacent skin acquired using different calibration schemes are shown in Figures 4.9(a)-(d). Figure 4.9(a) represents the image acquired by the system without any calibration scheme, where the quality of the image is degraded. Figure 4.9(b) represents the image obtained when the NNC algorithmbased calibration is implemented. The contrast of the obtained image is poor compared to the PTDI (sixth order) and proposed scheme, which are shown in Figures 4.9(c) and 4.9(d), respectively. However, the proposed algorithm gives an image with improved quality in comparison with the PTDI with higher order fitting and NNC, as demonstrated in figure 4.9(d). The performance of the proposed calibration is also validated by imaging the anterior chamber of an eye model (Carl Zeiss), which represents an optically transparent and dispersive medium with curved surface profile. Figure 4.9(e) represents the image of an eye model acquired by the OCT system without any calibration scheme, where the quality of the image is poor. Figure 4.9(f) demonstrates the quality of the image obtained when resampling based on the NNC algorithm was applied. The resolution at the deeper layers (iris and lens) is found to be degraded and more artifacts and aliasing effects are observed. Figures $4.9(\mathrm{~g})$ and $(\mathrm{h})$ represent the images when PTDI (sixth-order fitting) is used. Figure 4.9(h) shows the image with enhanced quality when the proposed scheme is implemented. The improved quality of the iris and lens shows the improvement in the resolution and the sensitivity at the deeper layers, which were degraded in the case of other schemes. 

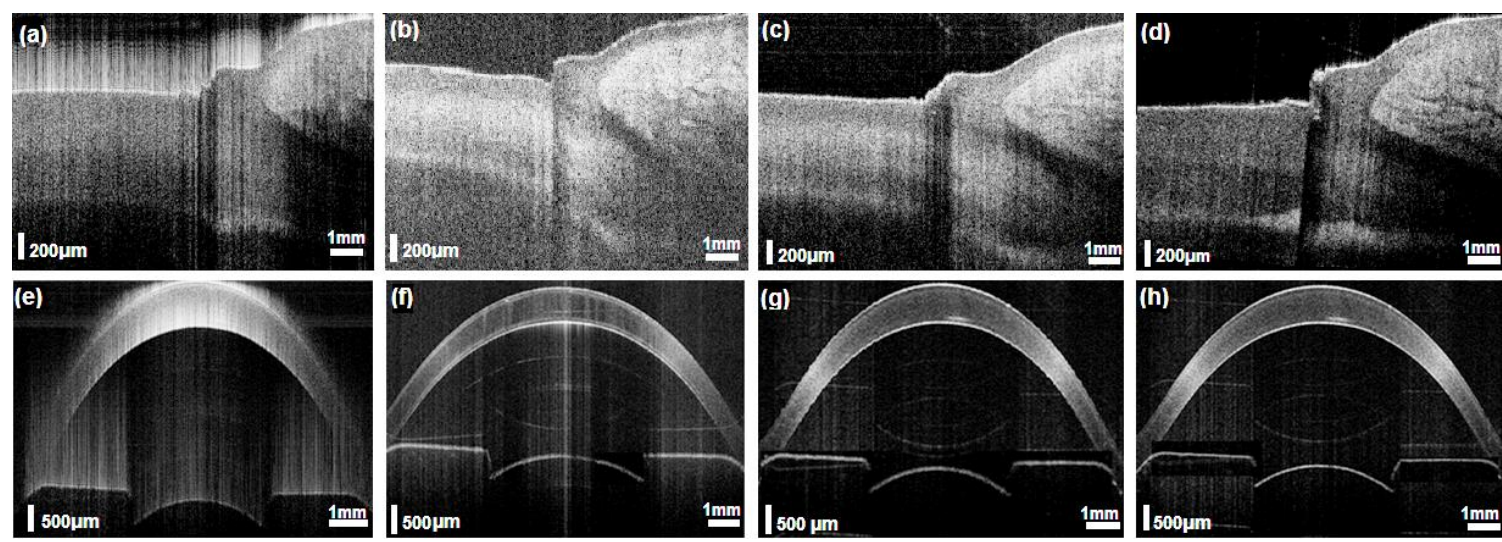

Figure 4.9 Images obtained under different calibration schemes. (a)-(d) Images of human finger nail and skin region; (e)-(h) images of an anterior chamber eye model; (a) and (e) without any calibration scheme; (b) and (f) using NNC; (c) and (g) using PTDI (sixth-order fitting); (d) and (h) using the proposed linearization scheme.

\subsection{Conclusion}

In summary, an accurate method for real-time calibration in SSOCT system is demonstrated. The proposed method performs automatic calibration by extracting the calibration signal from the OCT signal itself. This approach avoids the requirement for an auxiliary interferometer and an additional channel for calibration, thereby offering a costeffective solution for calibration. Moreover, the proposed method enables a compatible SSOCT configuration with minimal hardware features. In the proposed automatic scheme, the spectral component representing the sample surface reflection is separated using a narrow bandpass filter and used as the reference signal for calibration. The wavenumber linearization with an improved accuracy is achieved by a novel spectral phase-based direct-time domain interpolation scheme. The resampling function obtained by normalization and rescaling processes allows precise calibration irrespective of the nonlinearity of wavelength sweeping. The simple and computationally efficient algorithm used in the proposed method, allows real-time calibration of individual spectral interferograms based on their respective sweeping characteristics. Thus, the proposed method efficiently 
corrects the inaccuracy and instability associated with inter-sweep variance of the swept laser source.

The enhanced performance of the proposed scheme is validated in terms of axial resolution, sensitivity fall-off, computational time and quality of the reconstructed image. In comparison to the PTDI with polynomial fitting and NNC algorithm-based scheme, the proposed scheme improves the axial resolution and is found to correlate well with the theoretical value. Furthermore, the proposed method keeps the resolution almost constant along the entire imaging range. It is evident from the illustrated figures that the proposed scheme shows improved sensitivity and reduced sensitivity fall-off in comparison with the other schemes. The proposed calibration scheme being simpler and stronger in terms of computational efficiency was demonstrated to exhibit more than $30 \%$ improvement in the processing time when compared to the NNC and PTDI scheme with polynomial fitting.

Next chapter describes the theoretical and experimental investigations carried out for the contrast enhancement in OCT imaging using plasmonic nanoprobes. 


\section{Chapter 5: Nanoparticle-Assisted Contrast Enhancement in Optical Coherence Tomography}

This chapter begins with a detailed description of the fundamental theoretical aspects of contrast mechanism in OCT imaging, followed by the physics of surface plasmon resonance (SPR) in metallic nanoparticles. This is followed by theoretical and experimental investigations into the optical extinction properties of silver and gold nanoparticles and their capability for contrast enhancement in OCT imaging. A novel numerical approach is introduced for quantifying the extinction cross-section of nanoparticles with high absorption cross-section. The extinction cross-section calculated using OCT is compared with UV-Vis-NIR spectroscopy and finite-difference time-domain (FDTD)-based simulation results. Speckle-variance OCT imaging is demonstrated as a powerful tool to investigate the distribution of nanoparticles in biological tissues. The contrast enhancement capability of the proposed nanoparticles is demonstrated using phantom and pig adipose tissue samples, respectively.

\subsection{Introduction}

Optical coherence tomography (OCT) constructs tomographic images by detecting the depth-dependent back-reflected signal from morphological structures of the sample. Imaging at NIR wavelength such as $1300 \mathrm{~nm}$ allows the visualization of deeper structural morphology in biological tissues, which can improve existing and create new applications. Contrast of OCT image is fundamentally determined by the spatial variation of refractive indices. The reflectivity or intensity of the back-reflected signal depends on the difference 
between the refractive indices of the layers. Figure 5.1 shows a heterogeneous sample with two layers having different refractive indices $n_{1}$ and $n_{2}$. The power reflectivity of the boundary between two media $R_{S}$ is given by [150]

$$
\mathrm{R}_{\mathrm{s}}=\left|\frac{\mathrm{n}_{1}-\mathrm{n}_{2}}{\mathrm{n}_{1}+\mathrm{n}_{2}}\right|^{2}
$$

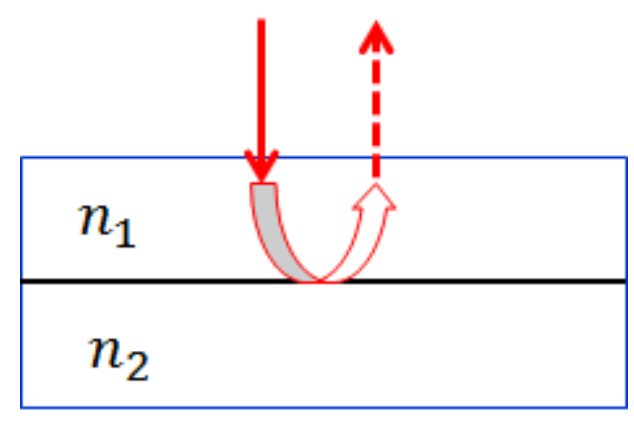

Figure 5.1 Reflection from the boundary between two media

From Equation (5.1), it can be understood that a smaller refractive index difference corresponds to a lower reflectivity. It is evident from Equation (2.2) that the amplitude of the balanced detected current is directly proportional to the sample reflectivity $R_{S}$. This means that OCT is unable to differentiate tissue layers or features with comparable refractive indices, particularly when the pathological tissue is optically or morphologically identical to normal tissue. The diagnostic capability of OCT is therefore limited at the earlier stages of diseases. The contrast of OCT images can be greatly enhanced by the administration of contrast agents. The unsurpassed capability of metallic nanoparticles for enhancing optical scattering and absorption due to the surface plasmon resonance (SPR), have made them a better choice for contrast enhancement in OCT. Plasmonicnanoparticles applied to a specific region can alter the amplitude of the back-scattered 
signal relative to its surroundings. Nanoparticles melded with OCT imaging can serve as multifunctional and highly sensitive diagnostic tools for subsurface imaging applications. Amongst the various kinds of metal nanoparticles, nanoparticles of gold and silver have provided significant contributions towards various fields of medicine [34]. Gold nanoparticles are widely used as a contrast agent because of their biocompatibility and non-toxicity. Silver nanoparticles have a higher extinction coefficient relative to gold nanoparticles of the same size and have been demonstrated in several bio-imaging applications [34, 151]. Coating silver nanoparticles with passive dielectric materials makes them more biocompatible and less cytotoxic.

\subsubsection{Surface plasmon resonance}

When the size of noble metals such as gold and silver are restricted to a nanoscale dimensions, they exhibit exciting properties different from the bulk. This is mainly due to the restrictions imposed towards the spatial confinement of the electrons, which induces restrictions to the electron motion. When such metallic nanoparticles are exposed to electromagnetic radiations, the conduction electrons in the metal nanoparticles will be displaced relative to the lattice points under the influence of electric field. The periodic displacement of the electric charges towards the opposite surface of nanoparticle creates electric dipoles. The dipole length depends on the composition, size, and shape of the nanoparticles. However, the Coulomb force of attraction between the opposite charges tends to restore them in equilibrium positions. This results in an electron oscillation with a specific frequency and the quantum of the electron oscillator is known as surface plasmon (SP). Under the irradiation of light, the surface plasmons will be coherently oscillating at a resonant frequency of the incident light, known as the SPR. At this resonant frequency, the 
amplitude of the oscillation reaches the maximum, giving rise to enhanced radiative and non-radiative properties. The resonant frequency and SPR intensity depend on many factors such as particle size, shape, structure, composition and the dielectric constant of the surrounding medium.

\subsubsection{Surface plasmon absorption, scattering and optical tuning}

The extinction (loss) of the electromagnetic wave after passing through a matter is mainly contributed by absorption or scattering. If the plasmon oscillation causes intra or inter-band transition of the electrons, the incident light will be strongly attenuated by the absorption. On the other hand, plasmon oscillation resulting in the emission of energy at the same frequency of incident light constitutes an elastic (Rayleigh) scattering. The extinction properties of plasmonic-nanoparticles were initially studied on gold nanospheres using Mie theory. The optical absorption and scattering is largely dependent on the size of the nanoparticles. The extinction cross-section of smaller nanoparticles is mainly caused by optical absorption [36]. As the nanoparticle size increases, Mie scattering begins to contribute to the total extinction. Therefore, nanoparticles with relatively large dimensions and higher scattering efficiency are preferred for imaging applications, whereas the smaller particles are mainly considered for photothermal application due to their high absorption cross-sections [152]. The high-absorption cross-section exhibited by the smaller nanoparticles allows heat conversion, leading to selective destruction of cell or tissue [36, 153].

For non-spherical nanoparticles such as nanorods, the SPR band intensity can be explained based on Gans theory $[154,155]$. According to Gans theory, the SPR bands in nanorods split into two modes: (a) longitudinal SPR mode corresponding to the electron oscillations 
along the length and (b) transverse SPR mode corresponding to the electron oscillations along the short axis of the rod (diameter). The plasmon wavelength of the longitudinal mode can be synthetically tuned across a broad spectral range covering the visible and NIR regions by tailoring their aspect ratio, whereas, the transverse band is insensitive to the size changes.

Besides altering the shape, optical tuning into NIR region can also be achieved by introducing structural variation into the nanoparticle such as in core-shell nanoparticles $[156,157]$. Metal core/silica shell nanoparticles are most widely used because of their inherent tuning property. The red shift of the SPR band from visible to NIR region can be achieved by varying the core-shell thickness ratio [156]. The red-shift is mainly due to the increased coupling between surface plasmons of the inner core and outer shell of the nanoparticle. Therefore, for the efficient usage of metallic nanoparticles for OCT applications, it is desirable to explain and quantify these optical behaviors using computational models. In this context, theoretical studies were conducted to address and develop FDTD method based computation models for silica-coated silver nanoparticles and nanorods with higher aspect ratio.

\subsubsection{Modelling and simulation using finite difference time domain method}

Finite difference time domain (FDTD) allows efficient computation of the electromagnetic fields and its propagation by solving Maxwell's equations discretely in time domain. In this approach, the computations are performed by dividing the time and space into discrete segments knows as Yee cells. These segmented rectangular-shaped cells must be small compared to the excitation wavelength. Time is quantized into small steps where each step represents the time required for the field to travel from one cell to the next. When many 
FDTD cells are combined together to form a three-dimensional volume, the result is an FDTD grid or mesh. By employing a light source into the computational model, it is possible to determine the propagation of electromagnetic field through the structure of interest until the electromagnetic fields are vanished from the computation region. The cell size is the most important constraint in any FDTD simulation since it determines not only the step size in time, but also the upper frequency limit for the calculation. In practice, the cell size must be sufficiently small for resolving dimensions and features of the structure to be simulated. The main advantage of the discrete time domain approach is that a single execution of the program can provide broadband output. Further, the FDTD approach has excellent scaling performance as the problem size grows. As the number of unknowns increase, the FDTD approach quickly outpaces other methods in efficiency. Furthermore, FDTD method was shown to be the most efficient approach for the analysis of field penetration into biological tissues.

\subsection{Investigation into contrast enhancement using Silica-Coated Silver Nanoplates}

Compared to other metallic nanoparticles, silver nanoparticles are particularly interesting because of their strongest plasmonic interaction with light. As matter of fact, the scattering cross-section of silver is greater than that of the other kinds of metals. Compared to other shapes, the anisotropic morphology of nanotriangles offers multiple LSPR bands with their

decreased symmetry [158]. Triangular nanoplates offer optical extinction peaks resulting from in-plane and out-of-plane modes, which can be conveniently tuned by controlling their aspect ratio (the ratio of edge length to thickness) [158, 159]. Since most of the signals are generated from tip area, nanotriangles provides excellent enhancement of the local field and the spatial resolution. The triangular-shaped nanoplates have been 
synthesized by various research groups with tight control over sizes and homogeneities that are suitable for bio-imaging applications $[158,160]$.

Coating silver nanoparticles with materials such as silica or polyethylene glycol (PEG) makes them more biocompatible and less cytotoxic $[160,161]$. Further, a core/shell configuration prevents agglomeration, and enhances the thermal and chemical stability of silver nanoparticles. Moreover, in-plane mode of the SPR can be red-shifted to the desired NIR region by changing the core-shell thickness ratio [160].

The following subsection illustrates the FDTD model and various simulations performed to determine the optical properties of the silica-coated silver nanoplates.

\subsubsection{FDTD model for LSPR Characterization in Silica-Coated Silver Nanoplates}

The optical response of the triangular-shaped silica-coated silver nanoplate (SSNP) was investigated using a computational model based on the FDTD method. 3-D FDTD computation models were formulated using FDTD Solutions 7.5 Commercial software (Lumerical Solutions Inc.) for a single nanoplate. The model involves the use of a total field scattered field (TFSF) source surrounding the defined nanoparticle, with one power monitor in the total field region and one power monitor in the scattered field region to calculate the absorption and scattering cross-sections, respectively. The analysis group placed inside the TFSF source calculates the absorption cross-section. The analysis group located outside the TFSF source calculates the scattering cross-section. In order to accurately resolve the electromagnetic interactions, a cubic grid with a grid spacing of 1 $\mathrm{nm}$ was used. The simulation was performed by choosing water as the embedding dielectric medium (refractive index=1.33). Figures 5.2(a) and 5.2(b) show the variation of 
resonance peak wavelength with the side length of SSNP and thickness of the silicacoating, respectively (for a silver base plate thickness of $10 \mathrm{~nm}$ ). Figure 5.2(a) shows that there is a significant redshift in the resonance peak wavelength with the increase in side length. On the other hand, increase in silica thickness results in slight red shift of resonance peak wavelength. Figure 5.2 illustrates that the triangular-shaped SSNP with a side length of $170 \mathrm{~nm}$, base silver thickness of $10 \mathrm{~nm}$, and silica shell thickness of $40 \mathrm{~nm}$ exhibits resonance peak at $1320 \mathrm{~nm}$. Figures 5.2(c) demonstrate the field enhancement in a triangular-shaped SSNP with the above mentioned dimensions, under LSPR. Figure 5.2 (d) shows that the spectral extinction plot obtained with the above physical dimensions.
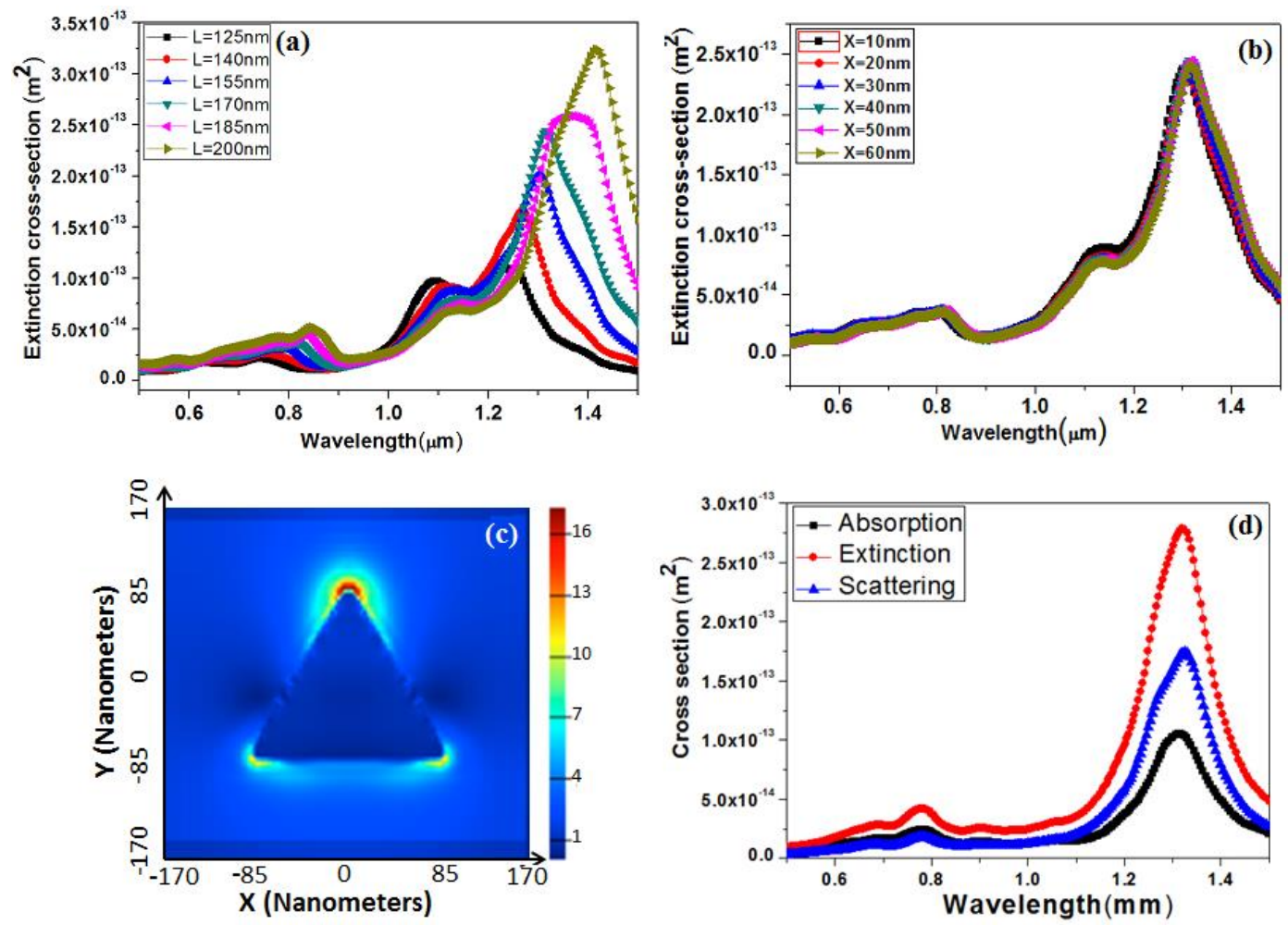

Figure 5.2 Variation of the resonance peak wavelength with (a) side length (b) thickness of silicacoating (c) enhancement of the electric field in the triangular-shaped SSNP. (b) extinction, scattering and absorption cross-section plot using the FDTD method. 
There are two major resonance peaks observed in the spectral plot. The large resonance peak which is red-shifted is due to the in-plane mode. The in-plane mode is generated by the oscillation of electrons along an axis parallel to the plane of the nanoplate (side length). The resonance peak with lower amplitude represents the out-of-plane mode, which is due to the electron oscillation along the axis perpendicular to the plane of nanoplate (thickness). Figure 5.2 (d) further shows that SSNP with the selected dimension gives a resonance peak at $1320 \mathrm{~nm}$. The obtained spectral plot gives an extinction-cross section of $2.7 \times 10^{-14} \mathrm{~m}^{2}$. The results also indicate that the scattering cross-section is dominant with respect to the absorption cross section. This predicts that SSNP as a promising scatteringbased contrast agent for OCT.

\subsubsection{Sample and Characterization}

The nanoplates were custom ordered from NanoComposix Inc., USA according to the dimensions used in the FDTD models. The obtained nanoplates have an average side length of $170 \pm 5 \mathrm{~nm}$. The average thicknesses of the base silver plate and silica shell are $10 \pm 1 \mathrm{~nm}$ and $40 \pm 2 \mathrm{~nm}$, respectively. Figure 5.3(a) shows transmission electron microscopy (TEM) images (JEOL1010, JEOL, USA) with the sizes and shapes of the SSNPs. The dimensions of the SSNPs were verified using a dynamic light scattering particle analyzer (Zetasizer, Melvern, UK). Figure 5.3 shows that the SSNPs exhibit excellent size uniformity. However, a $\pm 9 \%$ size variation was observed within 36 nanoplates. The SSNPs diluted in water have a mass concentration of $1 \mathrm{mg} / \mathrm{mL}$, which was estimated by using the inductively-coupled plasma spectroscopy (X Series 2 ICP-MS, Thermo-Fisher Scientific Inc., USA). 
A spectral extinction plot of SSNPs recorded using a UV-Vis-NIR spectrometer (8453 UV-Visible spectrometer, Agilent, USA) is shown in Figure 5.3(b). From the extinction plot, it is evident that the SSNPs exhibit a broad longitudinal resonance peak around 1300 $\mathrm{nm}$. The extinction coefficient of the SSNPs was calculated to be $30.45 \mathrm{OD} \mathrm{cm}^{-1} \mathrm{mg}^{-1} \mathrm{~mL}^{\text {at }}$ $1320 \mathrm{~nm}$, which is equivalent to $7.00 \mathrm{~mm}^{-1} \mathrm{mg}^{-1} \mathrm{~mL}$ (calculated using the following unit conversion formula: $1.00 \mathrm{OD} \mathrm{cm}^{-1} \mathrm{mg}^{-1} \mathrm{~mL}=0.23 \mathrm{~mm}^{-1} \mathrm{mg}^{-1} \mathrm{~mL}$ ). This corresponds to an extinction cross section of $2.48 \times 10^{-14} \mathrm{~m}^{2}$, which was calculated by dividing the extinction coefficient by the concentration of SSNPs.
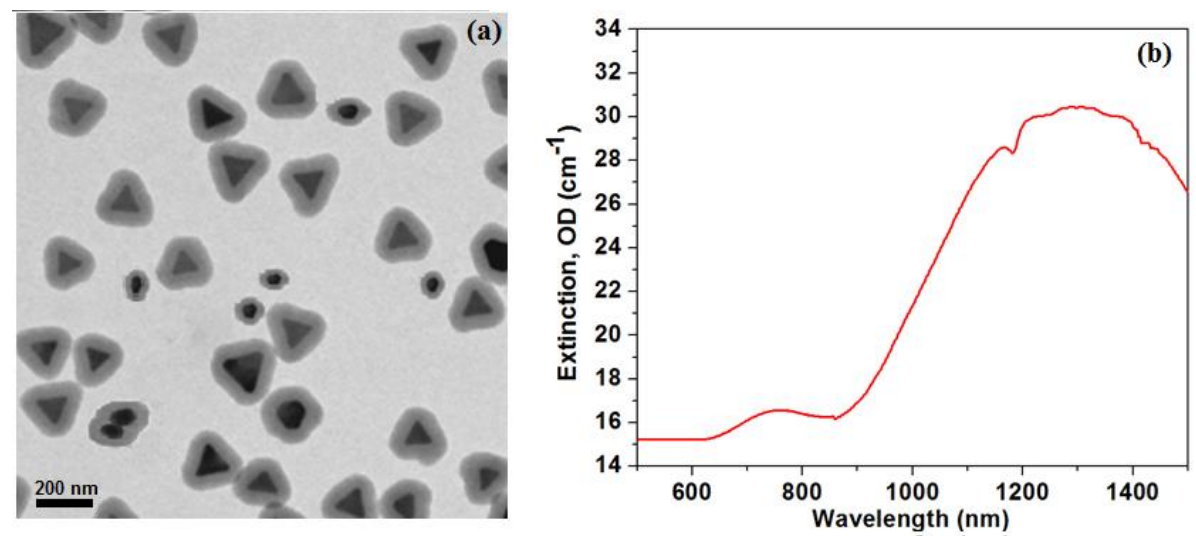

Figure 5.3 (a) TEM image of SSNPs (b) UV-Vis-NIR extinction spectrum recorded for SSNPs with a concentration of $1 \mathrm{mg} / \mathrm{mL}$.

\subsubsection{LSPR Characterization using OCT}

In order to estimate the optical extinction coefficient using OCT, SSNPs were filled in a flow cell channel with physical dimensions of $40 \mathrm{~mm}$ (length) x $10 \mathrm{~mm}$ (width) x $1 \mathrm{~mm}$ (depth), followed by scanning with OCT. Figure 5.4(a) shows a cross-sectional image of SSNPs filled in the flow channel. The two thick lines at the top of the image represent reflections from the top and bottom surfaces of the glass cover slip. Scattering caused by the SSNPs can be seen in Figure 5.4(a). 
Considering the single scattering model, the depth-dependent back-scattered signal acquired by the OCT system follows the Beer-Lambert law according to the relation [162]

$$
i_{s}(d) \propto \frac{\exp \left(-2 \mu_{t} z\right)}{\sqrt{\left(\frac{z-z_{f}}{z_{R}}\right)^{2}+1}}
$$

where $\propto$ means "proportional to", $z$ is the optical depth, and $\mu_{t}$ is the attenuation coefficient of the medium. Equation (5.2) shows that a back-scattered signal follows exponential decay versus imaging depth. Furthermore, the OCT signal intensity is affected by the confocal properties of the imaging optics (the confocal point spread function), which is represented by the denominator in the Equation (5.2). The terms $z_{f}$ and $z_{R}$ in Equation (5.2), represents the position of the confocal gate and the Rayleigh depth, respectively.
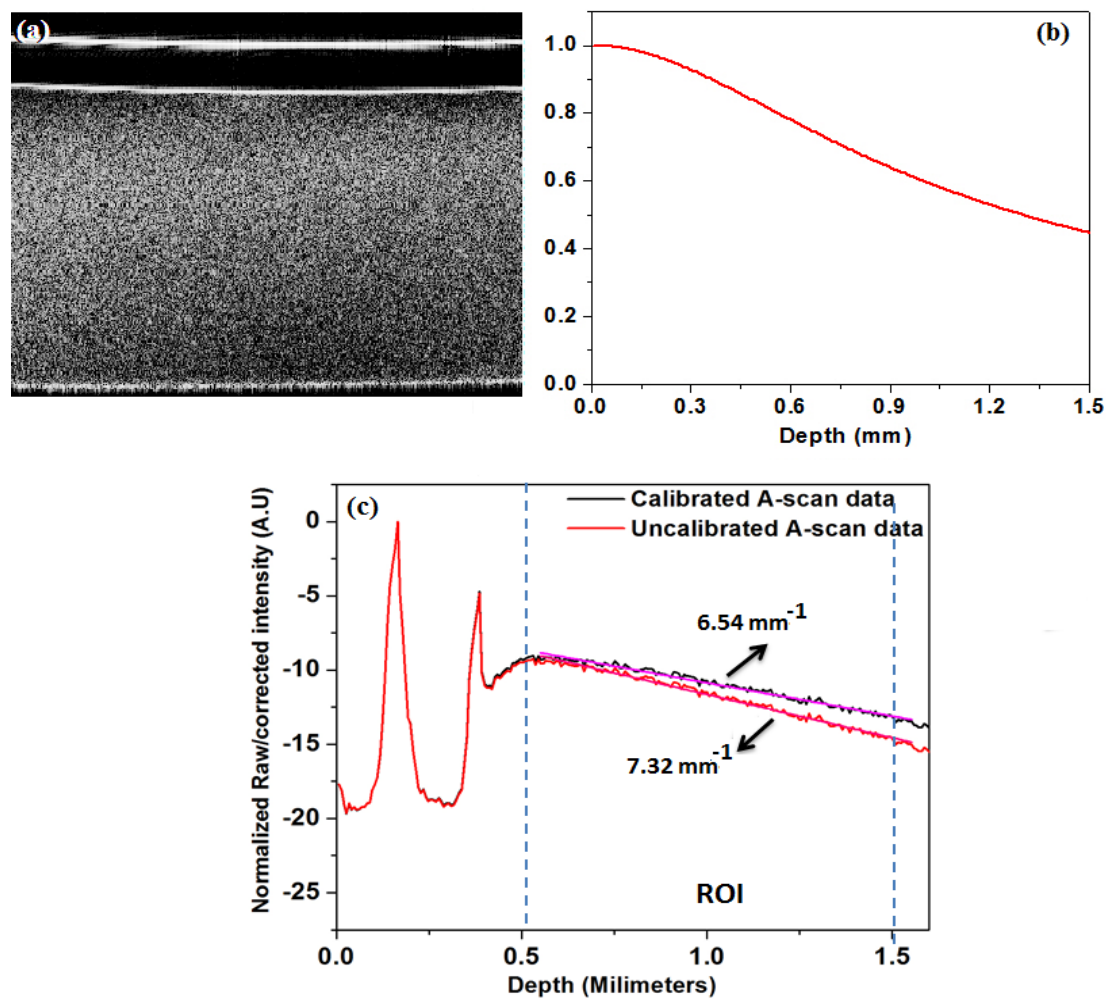

Figure 5.4 (a) Cross-sectional image of SSNPs filled in a flow channel. (b) Confocal point spread function (c) Normalized raw and calibrated A-scan data on a logarithmic scale. 
Figure 5.4 (b) represents the correction function defined by the axial confocal point function. The depth-dependent decay of the averaged intensity profile on a logarithmic scale is shown in Figure 5.4(c). The extinction coefficient can be calculated by fitting a straight line to the intensity profile using a least squares fitting approach. The corrected intensity plot is obtained by multiplying the correction function with the raw OCT signal as shown in Figure 5.4 (c). Accordingly, the extinction coefficients $7.32 \pm 0.05 \mathrm{~mm}^{-1}$ $\left(\mathrm{R}^{2}=0.99\right)$ and $6.54 \pm 0.05 \mathrm{~mm}^{-1}\left(\mathrm{R}^{2}=0.99\right)$ were obtained for the raw and calibrated A-scan profiles, respectively. The extinction coefficient $6.54 \mathrm{~mm}^{-1}\left(28.4 \mathrm{OD} \mathrm{cm} \mathrm{mg}^{-1} \mathrm{~mL}\right)$ calculated using the OCT technique corresponds to an extinction cross section of $2.32 \times 10^{-}$ ${ }^{14} \mathrm{~m}^{2}$. The extinction cross-section calculated by using the OCT scheme is in good agreement with the value obtained by UV-Vis-NIR spectroscopy.

The LSPR-induced extinction cross-section of the SSNPs characterized using the OCT technique was found to be reasonably consistent with the values obtained by UV-Vis-NIR spectroscopy and FDTD- based simulation. The extinction cross-section calculated using FDTD is ten-fold higher than the value obtained by OCT and UV-VIS spectroscopy. In addition, the extinction plot obtained by UV-Vis-NIR is broader than the spectral plot obtained by FDTD simulation. The major reasons for these differences are as follows. (1) FDTD-based simulation represents an ideal case, in which a single SSNP devoid of plasmonic coupling is considered. (2) The non-uniform geometry of the SSNPs and their random orientation alignment in water causes a reduction in their average extinction value. (3) The stacking of the nanoplates in the axial direction, or plates with a greater thickness decreases the aspect ratio (length/thickness). This eventually broadens the lower wavelength region (blue shift). (4) The lateral interaction of the nanoplates increases the aspect ratio leading to the broadening of the higher wavelength region (red shift). 
It is found that the estimated extinction cross-section of SSNPs $\left(2.32 \times 10^{-14} \mathrm{~m}^{2}\right)$ is six orders of magnitude higher than that of NIR dye molecules $\left(2.9 \times 10^{-20} \mathrm{~m}^{2}\right)$ and is comparable to that of gold nanorings $\left(4.86 \times 10^{-14} \mathrm{~m}^{2}\right)$ of a similar size $(174 \mathrm{~nm})$.

\subsubsection{Experimental investigation into contrast enhancement using SSNPs}

In order to investigate the contrast enhancement in biological tissues, SSNPs were delivered into fresh pig adipose tissue. The system generates cross sectional images with dimensions of $300 \times 300$ pixels representing, a field of view of $1.5 \times 2.4 \mathrm{~mm}$. The delivered SSNPs (5 $\mu \mathrm{L}$ droplets) were diffused through the tissue structures and occupied the intercellular substance. The tissue morphology mainly consists of adipose cells of relatively large size. During OCT scanning, LSPR-induced enhanced scattering in SSNPs contributes to the OCT signal.
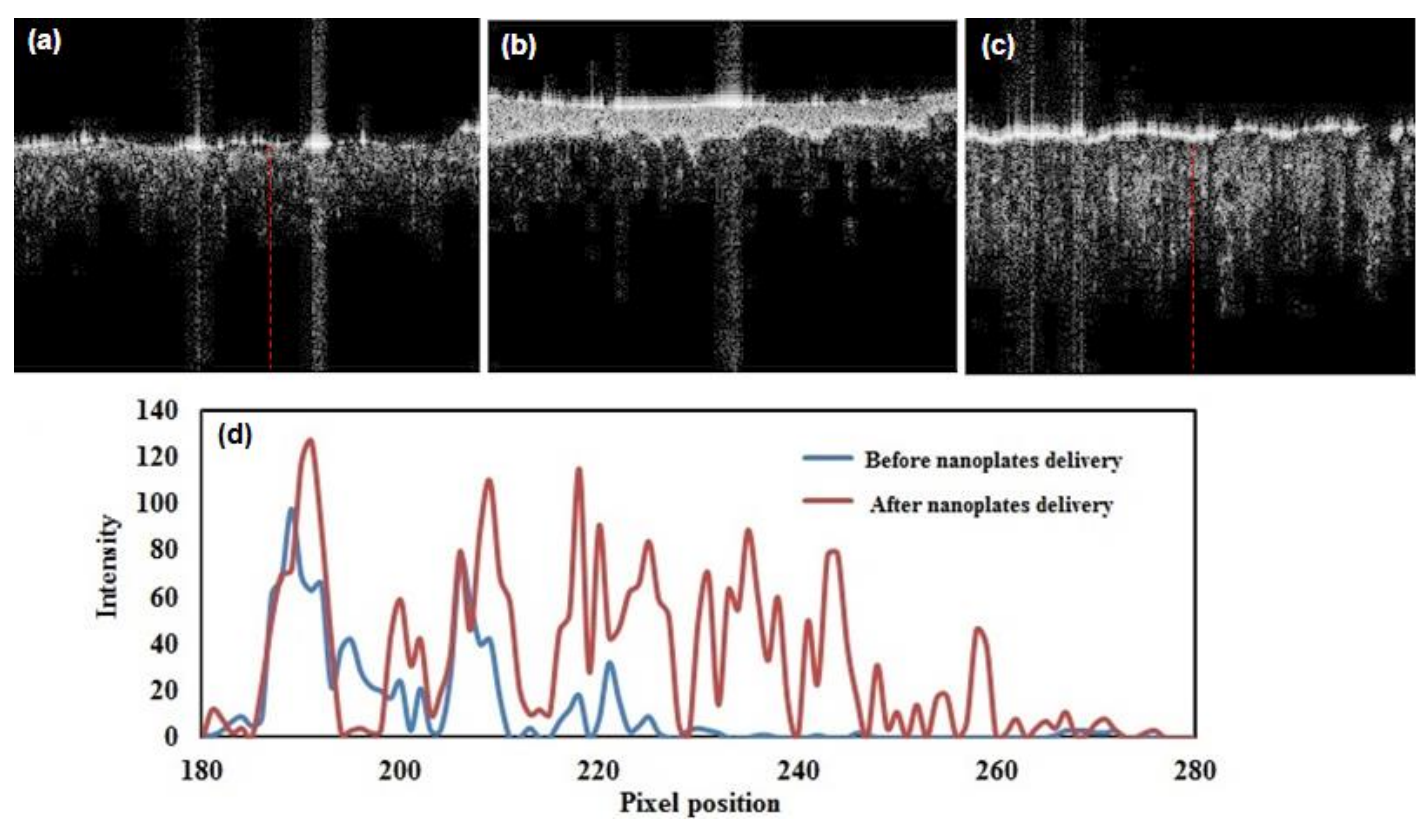

Figure 5.5 (a) Pig adipose tissue before applying nanoplates (b) Image of the tissue just after application of nanoplates droplet (c) Image of the tissue after the nanoplates are completely diffused (d) Variation of the pixel intensity along the marked (red) line. 
Figure 5.5 (a) shows an OCT image of fresh pig adipose tissue before the application of nanoplates, in which the deeper regions are not visible. The strong scattering on the tissue's surface shown in Figure 5.5 (b) represents the deposition of the SSNP droplet over the tissue sample. Figure 5.5 (b) shows that, the intensity of the subsurface region is significantly reduced. This is due to the strong optical extinction caused by the resonant SSNPs spread over the surface. Figure 5.5 (c) shows the image acquired after the SSNPs were completely diffused into the adipose tissue. The LSPR-induced enhanced scattering in SSNPs contributes to the OCT signal, leading to images with better contrast. Figure 5.5 (c) illustrates that the contrast of the image is enhanced and the visibility of the tissue microstructures is improved. Figure 5.5 (d) shows a variation in pixel intensity along the marked line before and after the delivery of nanoplates, demonstrating the enhanced image contrast after the delivery of SSNPs into the adipose tissue.
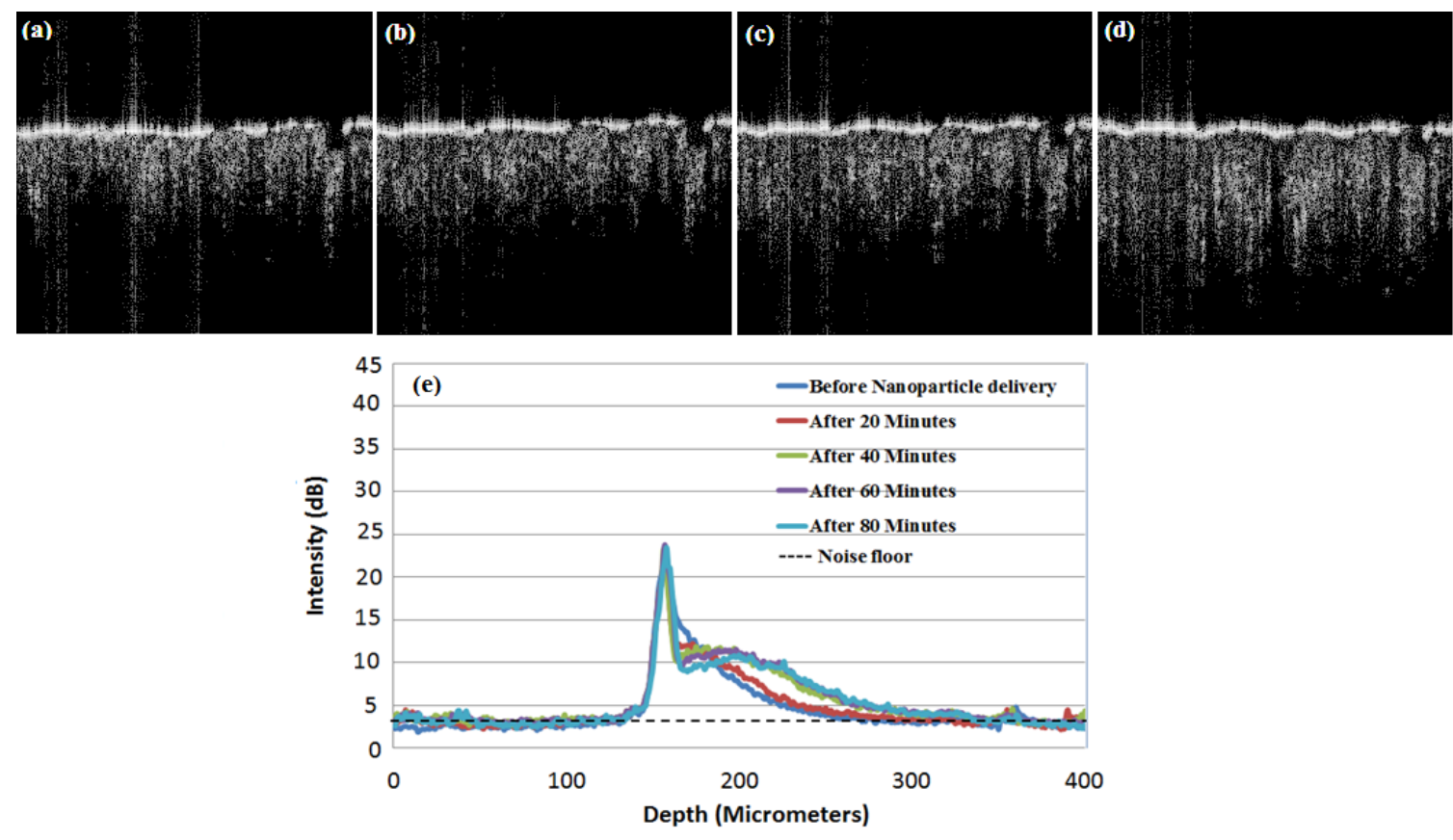

Figure 5.6 OCT images acquired at (a) 20 (b) 40 (c) 60, and (d) 80 min after the delivery of nanoplates (e) Average A-scan profiles obtained from B-scan data. 
In order to investigate the penetration of SSNPs into the adipose tissue, OCT intensity images were acquired at different time delays as shown in Figure 5.6. Figures 5.6 (a)-(d) represent the images acquired at 20, 40, 60, and $80 \mathrm{~min}$, respectively, after the application of the nanoplates. The figures show that the SSNPs are diffused into the deeper regions of the tissue sample, resulting in images with enhanced contrast. Figure 5.6(e) illustrates the average A-scan data obtained from a B-scan data before and after the application of SSNPs. It can be seen that, signal-to-noise ratio of the detected signal is improved after the delivery of the SSNPs into the tissue. Moreover, the relative shift in the peak observed in the decay profile with respect to time indicates the penetration of the SSNPs into the tissues.

\subsubsection{Speckle-variance imaging for the analysis of the nanoparticles distribution}

The term speckle is used to describe the OCT signal acquired as a result of partial coherently backscattered light from biological tissues. If an OCT image is acquired in a stationary object, the speckle pattern is temporally stationary as well. Due to the presence of moving particles in biological tissues (such as red blood cells), the speckle pattern varies with time and can be quantified by speckle variance calculations using either inter-frame or interline comparisons $[163,164]$. It is often difficult to investigate the distribution of SSNPs by differentiating the scattering contribution of SSNPs from the normal tissue scattering. The diffusion of SSNPs into the tissues would change the speckle patterns with respect to the static morphological tissue structures and increases the inter-frame pixel intensity variance at the respective locations. Therefore, speckle variance (SV) OCT images can be used for the efficient analysis of SSNP distribution. In addition, SV images are not affected by the confocal properties of the imaging optics. Figures 5.7(a)-(d) show 
SV images acquired before and after SSNP delivery into the adipose tissue. SV images are generated from four successive intensity images based on the following equation [164]

$$
S V_{i j k}=\frac{1}{N} \sum_{1}^{N}\left[\mathrm{I}_{i j k}(x, y)-\frac{1}{N} \sum_{1}^{N} \mathrm{I}_{i j k}(x, y)\right]^{2}
$$

where $i, j$ and $k$ represents the frame number, lateral pixel position, and axial pixel position of the B-scan image, respectively. $N$ is the number of B-scan images used for the variance calculation, and $\mathrm{I}_{i j k}(x, y)$ represents the intensity at pixel position $(x, y)$.

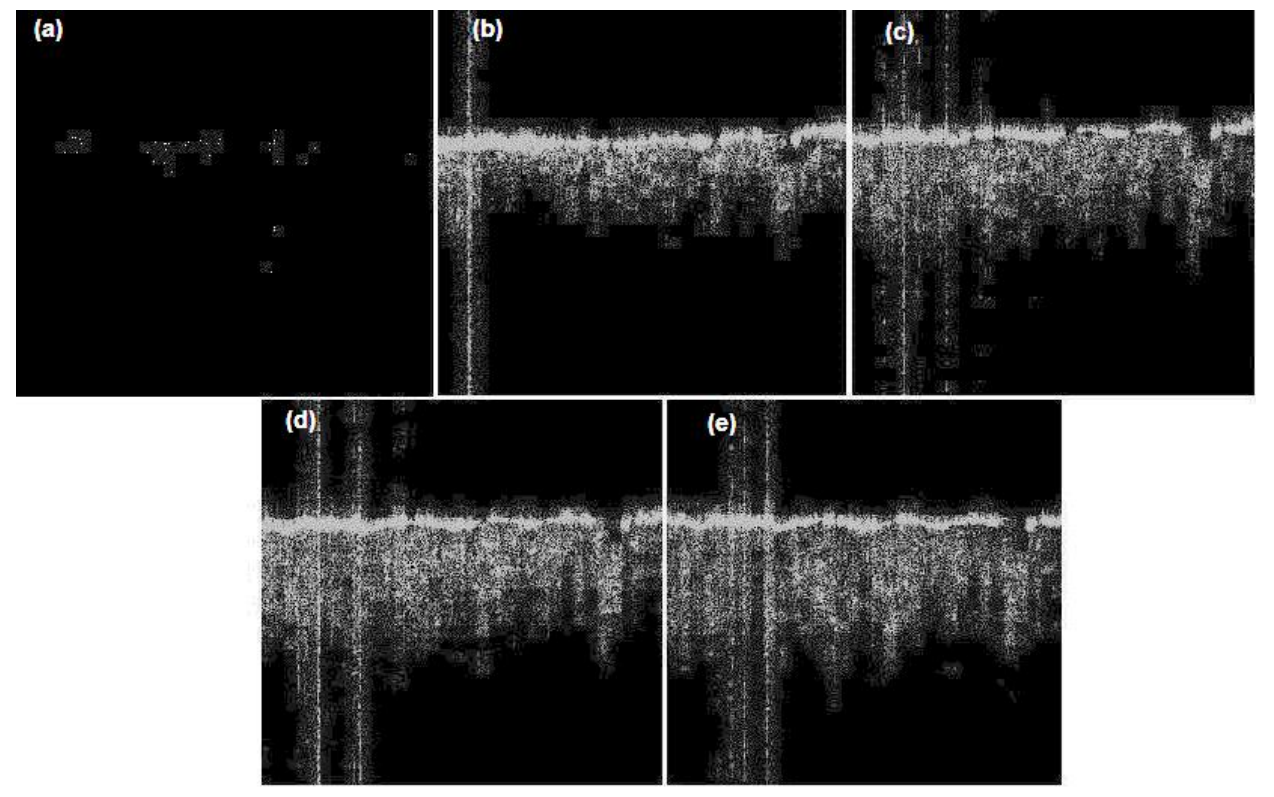

Figure 5.7 Speckle variance OCT images demonstrating the distribution of SSNPs (a) without SSNPs, and (b)-(e) 20, 40, 60, and 80 min after silver nanoplate delivery, respectively.

From Figure 5.7(a), it is clear that speckle variance in adipose tissue is negligible in the absence of SSNPs. Figures 5.7 (b) and (e) represent SV images generated at different time delays, showing the regions over which SSNPs are distributed. The figures show that the SSNPs penetrate into the deeper portions relative to the time, and are widely distributed 
over the subsurface region. This demonstrates the efficient delivery of SSNPs and their applicability over a large affected area.

\subsection{Gold nanorods with higher aspect-ratio as absorption-based contrast agent for OCT}

Gold nanorods (GNRs) are widely attractive for OCT imaging due to the tunability of their longitudinal plasmon band to NIR region. By changing the aspect ratio ( ratio of length to diameter) of GNRs, the longitudinal surface plasmon wavelength (LSPW) can be easily tuned to $1300 \mathrm{~nm}$ [154]. The total extinction as well as the scattering cross-sections of GNRs increases linearly with its effective size (effective radius, $R_{\text {eff }}$ ), although they are independent of the aspect ratio [36]. Similarly, a higher absorption cross-section can be achieved by choosing a smaller effective size. Therefore, GNRs with higher aspect ratios and smaller effective radii can act as efficient photoabsorption agents for imaging in the longer wavelength band. Additionally, their smaller effective sizes will allow efficient biodistribution and tissue clearance, making them suitable for in-vivo investigations.

The following subsection illustrates the FDTD model and various simulations performed to determine the optical properties of the GNRs with higher aspect ratio.

\subsubsection{FDTD model for LSPR characterization in gold nanorod}

A three dimensional FDTD computational model is formulated for gold nanorod as shown in Figure 5.8. The nanorod was composed of a solid cylinder whose ends were capped with solid hemispheres with radius equivalent to the radius of the solid cylinder. The optical constants for gold and silica were taken from Johnson and Christy, and palik, respectively [164]. FDTD method based computations were performed for determining extinctioncross-section as a function of aspect ratio. Figure 5.8(a) illustrates the variation of the longitudinal surface plasmon wavelength of GNR with aspect ratio. Figure 5.8 (a) shows 
that GNR with aspect ratio of 8.8 exhibits longitudinal peak at $1320 \mathrm{~nm}$. Figure 5.8 (b) shows the electric field enhancement around the GNR with a length of $88 \mathrm{~nm}$ and a diameter of $10 \mathrm{~nm}$.
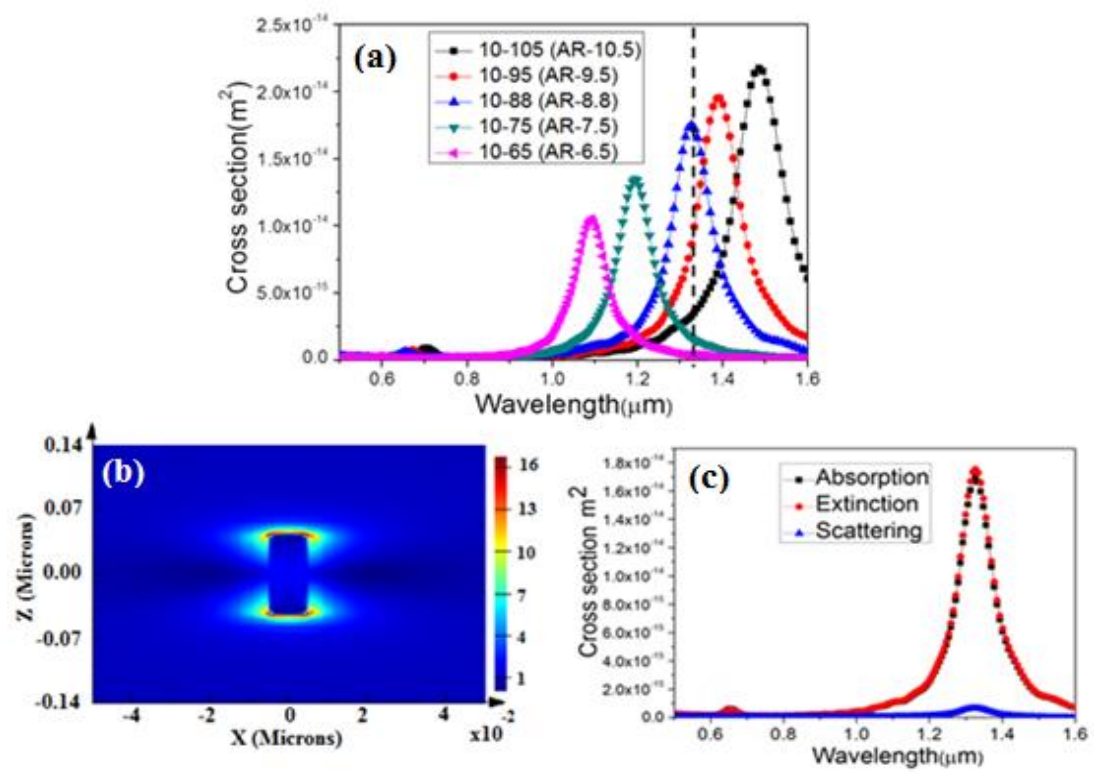

Figure 5.8 FDTD simulation results (a) Variation of LSPW with aspect ratio (b) Enhancement of the electric field due to the LSPR in GNR. (c) The scattering, absorption cross-section and total extinction plot.

The extinction spectrum of GNR shows two distinct peaks representing the longitudinal and transverse resonance modes. The longitudinal mode is due to the electronic oscillation along the length of the nanorod. This appears as the red-shifted component in the spectrum with large extinction peak. On the other hand, transverse mode is caused by the electronic oscillations along the width of the rod. This peak appears to be blue-shifted to lower wavelength regions with lower amplitude. From Figure 5.8 (c), the extinction crosssection corresponding to the longitudinal peak is found to be $1.75 \times 10^{-14} \mathrm{~m}^{2}$. The extinction spectrum further shows that, GNR has a higher absorption to scattering cross- 
section ratio $(\sim 10)$. This predicts the photoabsoption capability of the proposed GNRs and its potential for photothermal applications.

\subsubsection{Sample and Characterization}

Figure 5.9(a) shows transmission electron microscopy (TEM) images (JEOL1010, JEOL, USA) of the GNRs used in the study. The length and the diameter of the GNRs are measured to be $88 \pm 5 \mathrm{~nm}$ and $10 \pm 2 \mathrm{~nm}$, respectively. The GNRs were custom ordered from NanoComposix,.Inc.,USA according to the dimensions used in the FDTD models. The GNRs diluted in water has a weight concentration of $1 \mathrm{mg} / \mathrm{ml}$, when estimated using the inductively coupled plasma spectroscopy (X Series 2 ICP-MS, Thermo-Fisher Scientific Inc., USA). This corresponds to a number concentration of $6.86 \times 10^{11} / \mathrm{mL}$. Figure 5.9. (b) shows the extinction plot of GNRs recorded using UV-Vis-NIR spectroscopy (8453 UV-Visible spectrometer, Agilent, USA). The extinction plot shows that the longitudinal resonance peak of GNRs is at $1320 \mathrm{~nm}$. This is in good agreement with the FDTD based simulation result. The extinction coefficient of GNRs is calculated to be $33.43 \mathrm{OD} \mathrm{cm}^{-1} \mathrm{mg}^{-1} \mathrm{~mL}$ at $1320 \mathrm{~nm}$. This is equivalent to $6.56 \mathrm{~mm}^{-1} \mathrm{mg}^{-1} \mathrm{~mL}$. This gives an extinction cross-section of $1.12 \times 10^{-14} \mathrm{~m}^{2}$, which is obtained by dividing the extinction coefficient with the GNRs concentration.
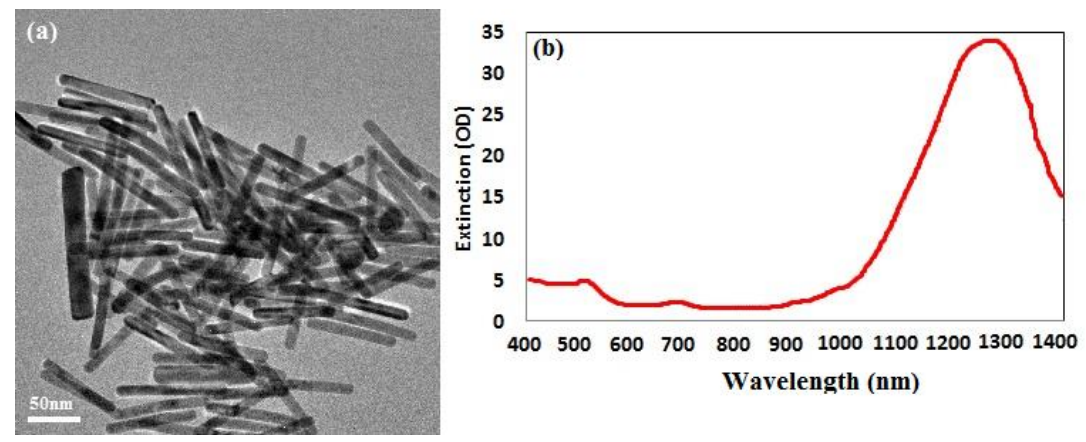

Figure 5.9 (a) TEM image of GNRs (b) Extinction spectrum obtained via UV-Vis-NIR spectroscopy 


\subsubsection{Estimation of extinction cross-section using OCT}

The method demonstrated in the section 5.2.3 is not suitable for measuring the extinction coefficient of highly absorbing nanoparticles due to the immediate decay of the A-scan signal. Alternatively, the extinction coefficient can be accurately determined using crosscorrelation method, in which the OCT signal from a reference phantom and GNR embedded phantoms are considered. The phantom was made of agar embedded with $\mathrm{TiO}_{2}$ nanoparticles to mimic biological sample (Refer Appendix B). Figures 5.10 (a) and 5.10 (b) represent the OCT images of the reference and GNR deposited phantoms, respectively. Figure 5.10 (b) shows that the intensity of the image is significantly reduced due to the strong absorption caused by the distribution of GNR. Figure 5.10 (c) represents the averaged A-scan data obtained from reference and GNR deposited phantom.
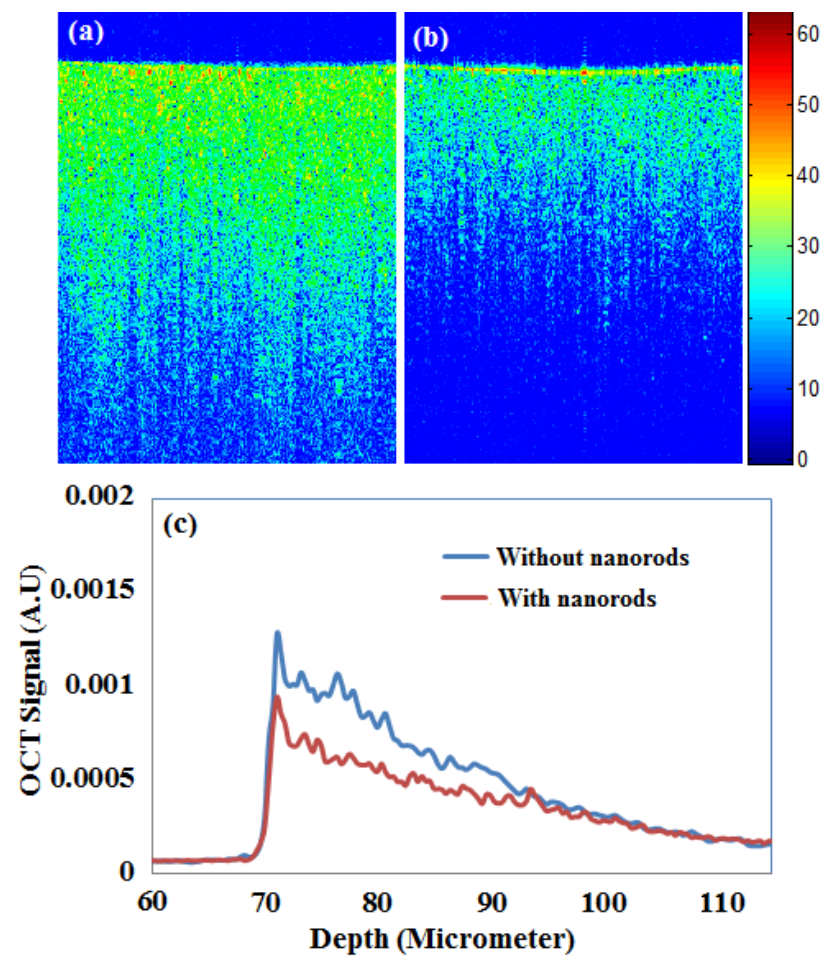

Figure 5.10 OCT image of agar gel phantom embedded with $\mathrm{TiO}_{2}$ (a) Phantom without GNRs (b) Phantom with GNRs (c) Averaged A-scan data with and without GNRs. 
In order to measure the extinction-cross section, the single scattering model is considered. If there is no multiple scattering, the depth-dependent back scattered signal $\left(S_{O C T}(z)\right)$ acquired by the OCT system follows Beer-Lambert law according to the relation [162]

$$
S_{O C T}(z)=S_{0} K\left(\mathrm{z}-z_{f}\right) \sqrt{\mu_{b}} \exp \left(-2 \mu_{t} z\right)
$$

Where $S_{0}$ represents the background OCT signal. $K\left(\mathrm{z}-z_{f}\right)$ represents the normalized calibration function that corrects the confocal properties of the sample arm optics. $\mathrm{z}$ and $z_{f}$ represents the arbitrary depth position and the position of the focus, respectively. Similarly, $\mu_{b}$ and $\mu_{t}$ represent the back scattering coefficient and total extinction coefficient, respectively.

The extinction coefficient of the sample specimen can be derived from the signal values corresponding to the two arbitrary depth positions $z_{1}$ and $z_{2}$ in the exponential decay region. Let $S\left(z_{1}\right)$ and $S\left(z_{2}\right)$ represent the signal values at $z_{1}$ and $z_{2}$, respectively. Using Equation (5.4), the extinction coefficient can be derived as,

$$
\begin{aligned}
& S\left(z_{1}\right)=S_{0} K\left(\mathrm{z}_{1}-z_{f}\right) \sqrt{\mu_{b}} \exp \left(-2 \mu_{t} z_{1}\right) \\
& S\left(z_{2}\right)=S_{0} K\left(\mathrm{z}_{2}-z_{f}\right) \sqrt{\mu_{b}} \exp \left(-2 \mu_{t} z_{2}\right)
\end{aligned}
$$

Take natural logarithm (ln) on both sides of the equation (2) and (3)

$$
\begin{gathered}
\ln \left[S\left(z_{1}\right)\right]=\ln \left(S_{0}\right)+\ln \left[K\left(\mathrm{z}_{1}-z_{f}\right)\right]+\frac{1}{2} \ln \left(\mu_{b}\right)-2 \mu_{t} z_{1} \\
\ln \left[S\left(z_{2}\right)\right]=\ln \left(S_{0}\right)+\ln \left[K\left(\mathrm{z}_{2}-z_{f}\right)\right]+\frac{1}{2} \ln \left(\mu_{b}\right)-2 \mu_{t} z_{2}
\end{gathered}
$$

Equation (5.8) - Equation (5.7) 


$$
\ln \left[S\left(z_{1}\right)\right]-\ln \left[S\left(z_{2}\right)\right]=\ln \left[K\left(\mathrm{z}_{1}-z_{f}\right)\right]-\ln \left[K\left(\mathrm{z}_{1}-z_{f}\right)\right]+\mu_{t} 2\left(z_{2}-z_{1}\right)
$$

Therefore the extinction coefficient $\mu_{t}$,

$$
\mu_{t}=\frac{1}{\Delta z}\left[\ln \frac{S\left(z_{1}\right)}{S\left(z_{2}\right)}-\ln \frac{K\left(\mathrm{z}_{1}-z_{f}\right)}{K\left(\mathrm{z}_{1}-z_{f}\right)}\right]
$$

Where $\Delta \mathrm{z}=2\left(\mathrm{z}_{2}-\mathrm{z}_{1}\right)$

Based on Equation (6.0), extinction coefficient reference phantom (Without GNRs) $\left(\mu_{\operatorname{Re} f}\right)$ can be expressed as

$$
\mu_{\operatorname{Re} f}=\frac{1}{\Delta z}\left[\ln \frac{S_{\operatorname{Re} f}\left(z_{1}\right)}{S_{\operatorname{Re} f}\left(z_{2}\right)}-\ln \frac{K\left(\mathrm{z}_{1}-z_{f}\right)}{K\left(\mathrm{z}_{1}-z_{f}\right)}\right]
$$

Similarly for phantom with GNRs $\left(\mu_{\operatorname{Re} f+G N R}\right)$, extinction coefficient can be expressed as

$$
\mu_{\operatorname{Re} f+G N R}=\frac{1}{\Delta z}\left[\ln \frac{S_{\operatorname{Re} f+G N R}\left(z_{1}\right)}{S_{\operatorname{Re} f+G N R}\left(z_{2}\right)}-\ln \frac{K\left(\mathrm{z}_{1}-z_{f}\right)}{K\left(\mathrm{z}_{1}-z_{f}\right)}\right]
$$

Therefore, the extinction coefficient of GNRs $\left(\mu_{G N R}\right)$ is given by

$$
\mu_{G N R}=\mu_{\operatorname{Re} f+G N R}-\mu_{\operatorname{Re} f}=\frac{1}{\Delta z}\left[\ln \frac{S_{\operatorname{Re} f+G N R}\left(z_{1}\right)}{S_{\operatorname{Re} f+G N R}\left(z_{2}\right)}-\ln \frac{S_{\operatorname{Re} f}\left(z_{1}\right)}{S_{\operatorname{Re} f}\left(z_{2}\right)}\right]
$$

This approach allows a fast and accurate estimation of the extinction coefficient from the A-scan data without any need of linear fitting. Moreover, the effect of confocal properties of the imaging optics will be automatically corrected in this method. For convenience, OCT signal values corresponding to the depth positions $z_{1}=80 \mu \mathrm{m}$ and $z_{2}=90 \mu \mathrm{m}$, near to the center of exponential decay region of the A-scan data are considered for calculation. For the reference phantom, the signal values are found to be $S_{R e f}\left(z_{1}\right)=0.0008$ and 
$S_{R e f}\left(z_{2}\right)==0.000543$, respectively. In the case of GNRs embedded phantom, the obtained values are $S_{R e f+G N R}\left(z_{1}\right)=0.000547$ and $S_{R e f+G N R}\left(z_{2}\right)=0.000421$, respectively. This gives an extinction coefficient of $6.325 \pm 0.012 \mathrm{~mm}^{-1} \mathrm{mg}^{-1} \mathrm{~mL}$ (or 27.36 $\pm 0.02 \mathrm{OD} \mathrm{cm}^{-1} \mathrm{mg}^{-1} \mathrm{~mL}$ ) based on Equation (6.3). This is corresponding to an average extinction cross-section of $1.09 \times 10^{-14} \mathrm{~m}^{2}$.

\subsubsection{Results and Discussion}

The above analysis shows that the estimated extinction cross-section of GNRs using OCT is consistent with the results obtained via UV-Vis-NIR spectroscopy and FDTD-based simulation. Table 1 compares the extinction properties of GNRs with other contrast agents reported for OCT imaging around $1300 \mathrm{~nm}$.

Table 5.1 Optical extinction properties of different contrast agents

\begin{tabular}{|c|c|c|c|c|}
\hline $\begin{array}{c}\text { Type of OCT } \\
\text { contrast agent }\end{array}$ & $\begin{array}{c}\text { Characteristic } \\
\text { dimension } \\
(\mathbf{n m})\end{array}$ & $\begin{array}{c}\text { OCT } \\
\text { wavelength } \\
(\mathbf{n m})\end{array}$ & $\begin{array}{c}\text { Extinction } \\
\text { cross- } \\
\text { section } \\
\mathbf{\sigma}_{\text {Ext }}\left(\mathbf{m}^{2}\right)\end{array}$ & $\begin{array}{c}\text { Absorption } \\
\left(\boldsymbol{\sigma}_{\text {Abs }}\right) \text { to } \\
\text { scattering } \\
\text { cross- } \\
\text { section } \\
\text { ratio }\end{array}$ \\
\hline $\begin{array}{c}\text { Indocyanine } \\
\text { green[125] }\end{array}$ & single & 800 & $2.9 \times 10^{-20}$ & $\sigma_{\text {Ext }}=\left(\sigma_{\text {Abs }}\right)$ \\
\hline $\begin{array}{c}\text { Gold } \\
\text { nanoshell[128] }\end{array}$ & 291 & 1310 & $2.21 \times 10^{-13}$ & 0.34 \\
\hline $\begin{array}{c}\text { Gold } \\
\text { nanorings[166] }\end{array}$ & 174 & 1310 & $4.86 \times 10^{-14}$ & 0.47 \\
\hline $\begin{array}{c}\text { Silica coated } \\
\text { silver } \\
\text { nanoplates }\end{array}$ & 170 & 1320 & $2.32 \times 10^{-14}$ & 0.65 \\
\hline $\begin{array}{c}\text { Gold } \\
\text { Nanorods }\end{array}$ & 1320 & $1.09 \times 10^{-14}$ & 10 \\
\hline
\end{tabular}


Table 5.1 shows that the extinction cross-section of GNRs is six orders of magnitude higher than that of NIR fluorescent molecule. Compared to gold nanoshells and nanorings, GNRs exhibit a higher absorption to scattering cross-section ratio $(\sim 10)$, thereby suggesting them as a potential candidate for absorption based contrast agents and around $1300 \mathrm{~nm}$. Similarly, the effective size of GNRs is much smaller than that of gold nanoshells and gold nanorings. This makes the proposed GNRs, more suitable for in-vivo imaging as well as photothermal applications around $1300 \mathrm{~nm}$. A qualitative investigation of the photothermal effect of the proposed GNRs using OCT is presented in the Appendix C. It has to be noted that GNRs, show comparable extinction cross-section with gold nanorings and SSNPs, even though their size is much smaller than that of nanorings and SSNP.

\subsection{Conclusion}

In summary, silica-coated silver nanoplates (SSNPs) and gold nanorod (GNR) with higher aspect ratio are demonstrated as powerful contrast agents for optical coherence tomography around $1300 \mathrm{~nm}$. Experimental and simulation studies show that SSNPs with the selected dimension exhibit higher scattering to absorption cross-section ratio. The LSPR induced extinction cross-section of the SSNPs is measured using the OCT technique based on a flow-cell based experiment. The extinction cross-section measured using OCT technique is reasonably consistent with the values obtained by UV-Vis-NIR spectroscopy and FDTD based simulation. It is found that SSNPs exhibit a higher extinction crosssection comparable with that of gold nanorings. The experimental results show that the LSPR-induced scattering in SSNPs enhances the OCT signal and enable their detection with high sensitivity. The experimental results also show that image contrast is greatly 
enhanced and deeper layers are clearly visible after the delivery of the SSNPs. The timeresolved intensity images and average A-scan profile showed the efficient transport of SSNPs into the deeper regions of the tissues. The speckle variance imaging is demonstrated as a powerful tool to investigate the distribution of SSNPs in biological tissues. The acquired speckle-variance images show that SSNPs are efficiently distributed over the targeted tissue region, demonstrating their applicability in a large lesion area.

Gold nanorods (GNRs) with an aspect ratio of 8.8 are demonstrated as absorption based contrast agents for OCT imaging and photothermal applications around $1300 \mathrm{~nm}$. GNRs with a length of $88 \pm 5 \mathrm{~nm}$ and a diameter of $10 \pm 2 \mathrm{~nm}$ exhibit LSPR induced extinction peak at $1320 \mathrm{~nm}$ wavelength. The FDTD simulations and phantom experimental studies show that GNRs exhibit excellent absorption to scattering cross-section ratio. Optical extinction of GNRs is estimated using OCT based on a cross-correlative approach. It is shown that extinction cross-section calculated using OCT is consistent with the FDTDbased simulation and spectroscopy results. The potential of GNRs as absorption based contrasts agent for OCT has been demonstrated using a gel-phantom based study.

Next chapter details the work performed to investigate the potential of the OCT for realtime in-situ imaging of bacterial biofilms and to explore the metrological capability of OCT to assess different optical and physical parameters of the biofilms. 


\section{Chapter 6: Real-time in-situ investigation of bacterial biofilms using optical coherence tomography}

This chapter describes the use of optical coherence tomography for high resolution in-situ imaging of the development of bacterial biofilms. Chapter begins with the in-situ investigation of biofilms of Pseudomonas Aeruginosa (PA-1) using cross-sectional images. This will be followed by the theoretical and experimental demonstration of a novel methodology that allows simultaneous measurement of group refractive index and the physical thickness biofilms. The experimental investigations are further extended towards imaging microcolonies of Klebsiella Pneumonia (KP-1) using swept source based optical coherence microscopy (SSOCM). The development of microcolonies and the transient processes in response to the antibacterial treatment were investigated using enface images. The real-time in-situ quantification of optical density and planar density of biofilms was performed using novel methodologies based on OCM technique and compared with the measurements obtained via standard methods.

\subsection{Introduction}

Biofilms are aggregates of microbial communities growing on living and inert surfaces, frequently enclosed in matrix formed by the extra cellular polymeric substances (EPS). The major contents of the biofilm mass are contributed by water (up to 97\%), microbial cells $(2-5 \%)$ and the extra cellular polymeric substances (EPS) (1-5\%) [133]. Biofilms are widely found in nature and often characterized by their structural organization and the population density. The structural organization of the microbial communities is significantly influenced by the various environmental gradients such as shear stress, 
temperature and the nutrient composition $[132,166]$. Biofilms are also observed in industrial and medical environments often giving rise to a wide range of concerns, especially biosafety. It has been reported that biofilms can develop on or within the indwelling medical devices such as catheters, needleless connectors, contact lenses and mechanical joints and various body tissues such as teeth, middle ear, oral tissues etc [168, 169]. The biofilms colonized in such devices are identified as the major reason for the healthcare-associated bacterial infections $[170,171]$. The more problematic drug-resistant bacterial biofilms encountered today include Pseudomonas aeruginosa (PA-1) and Klebsiella pneumoniae (KP-1) $[145,168]$. KP-1 species are identified as the major contributor of the nosocomial infections and medically most important species of the genus $[172,173]$. Despite the wide distribution of PA-1 in nature and the potential for community-acquired infections, serious infections with PA-1 are predominantly hospital acquired. The recent studies show that PA-1 is the second most common cause of nosocomial pneumonia, health care-associated pneumonia [174]. The formation of biofilm and EPS enable these microorganisms to achieve better resistance towards the antimicrobial treatment as well as tenaciously attaching to surfaces. Hence it is often a challenge to eradicate biofilms from the biomedical and industrial environments in order to meet sufficient biosafety level. In order to have a better understanding and control of biofilms in highly sensitive environments, an efficient in-situ measurement/imaging scheme is required that would assist the strategies used for eradicating the biofilms. Besides the medical environments, biofilms are cultivated in laboratories under defined conditions for research purposes. In order to characterize the biofilm growth in terms of structural organization and population density, monitoring of biofilms and measurement of 
parameters such as thickness, refractive index and the flow patterns have to be performed nondestructively.

The capability of OCT to perform label free imaging allows efficient monitoring of biofilms and their development in biologically sensitive environments. The high resolution, depth-resolved cross-sectional images rendered by OCT reveal the structural information of bacterial biofilms. Further, OCT provides the combined spatial and temporal resolution necessary for assessing the acute biofilms to consistent growth and perturbations. Apart from bio-imaging applications, the applicability of OCT can be extended to metrological investigations because of the inherent capability of optical interferometry to perform precise measurement with high sensitivity. The following subsection describes the potential of the OCT for in-situ imaging of PA-1 biofilm and methodology for the quantification of refractive index and thickness.

\subsection{In-situ investigation of biofilms of Pseudomonas Aeruginosa}

PA-1 is a ubiquitous organism present in many diverse environmental settings, and it can be isolated from various living sources, including plants, animals, and humans. PA-1 biofilm generally appears as structured community and forms relatively thick film on the surface [175]. The PA-1 biofilm developed in a flow cell is used for the study, which is representative of a wide range of scenarios where biofilms develop. The mean biofilm thickness provides a measure of the spatial size of the biofilm and is the most common variable used in biofilm literature to quantify the growth. 


\subsubsection{Experiments and operational conditions}

The experimental setup for the biofilm cultivation and monitoring using the OCT instrument is shown in Figure 6.1. The three channel flow cell with channel dimension $1 \times 4 \times 40 \mathrm{~mm}^{3}$ was inoculated with PA-1 set at an optical density $\left(\mathrm{OD}_{600}\right)$ of 0.1 . This corresponds to about $1 \times 10^{8} \mathrm{cfu} / \mathrm{ml}$. Lysogeny broth (LB) (Miller) media was supplied at a flow rate of $10 \mathrm{ml} /$ hour with a temperature at $22^{0} \mathrm{C}$. A glass cover slip is used to cover up the biofilms grown in flow cells, through which the OCT monitoring is performed.

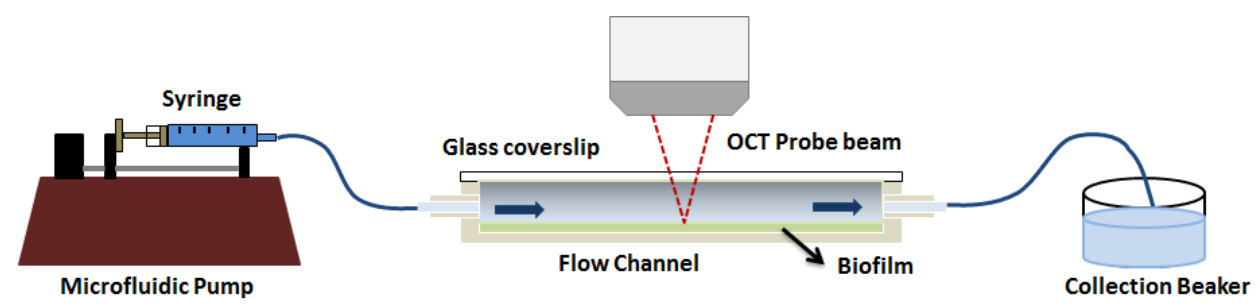

Figure 6.1 Flow cell channel used for the biofilm growth and imaging using OCT

The in-situ imaging was performed using the SSOCT system which is described in the Chapter 3. The OCT system consist of telecentric lens (LSM 03, Thorlabs, USA) with optical zoom of $5 \mathrm{X}$ which provides a maximum scanning area of $100 \mathrm{~mm}^{2}$. Since there is a considerable amount of spatial heterogeneity in the flow channels, the images were acquired at bottom positions of the flow cell channel at distance of $5 \mathrm{~mm}$ from the inlet. This minimizes the structural heterogeneities caused by the spatial differences in the flow channel. The OCT engine was configured to provide high resolution images with a sensitivity of $115 \mathrm{~dB}$ at $16 \mathrm{kHz}$ A-scan rate. The lateral and the axial resolution of the developed SSOCT system are $18 \mu \mathrm{m}$ and $8 \mu \mathrm{m}$ (in air) respectively. The working distance between the scanning lens and the cover glass slip is manually adjusted such that the focus 
of the beam was placed approximately $500 \mu \mathrm{m}$ above the bottom surface of the flow cell in order to optimize visualization of features at both the bottom and top surfaces. In order to acquire the cross-sectional images of biofilm, an OCT probe signal is scanned over a length of $9 \mathrm{~mm}$ along the flow cell. This gives an image dimension of $500 \times 250$ pixels corresponding to a physical depth of $1.5 \mathrm{~mm}$.

\subsubsection{Methodology for the simultaneous measurement of refractive index and thickness of biofilm}

The optical path length of a sample is given by the product of the physical length of the sample and its refractive index. In order to determine the thickness and the refractive index, the sample (BC) is placed between two reference reflectors (or glass plate or any reflective surface) as shown in Figure 6.2, followed by the acquisition of the A-scan signal using OCT.

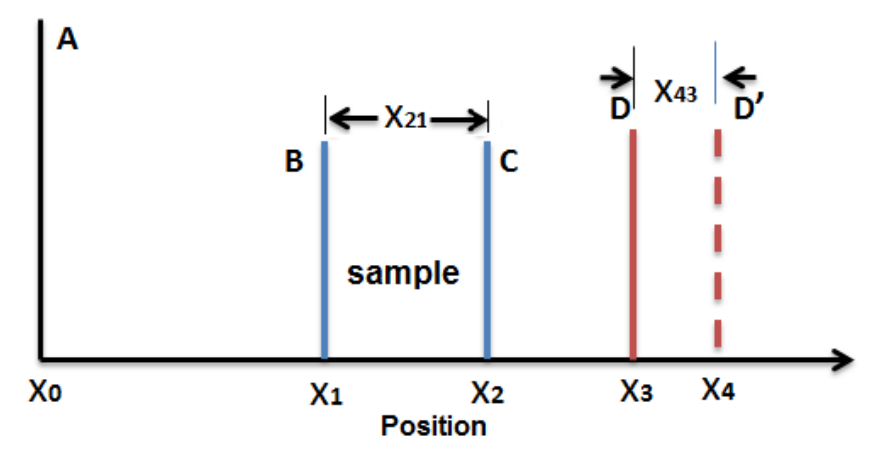

Figure 6.2 The positions of the sample and the reference reflectors

Initially, the reflections from the reference surfaces are measured in the absence of sample. As shown in Figure 6.2, $\mathrm{X}_{0}$ and $\mathrm{X}_{3}$ are the locations of two reference reflectors (A and D) which are obtained in the absence of sample medium. The position of the reference surfaces are represented by the point spread functions, which would be obtained by 
performing inverse Fourier transformation of the fringes. Later, the positions of the reference surfaces are measured by inserting the sample between the reference surfaces. In this case the position of the reference surface behind the sample (D) would be apparently shifted (D') due to the path length difference induced by the refractive index of the sample. The shifted position is represented by $\mathrm{X}_{4}$ in the Figure 6.2. In both cases, the reference surface A has no influence on the measurement since its position would not be altered by the presence of sample. However, the position of the surface A would be helpful in determining the relative shift in the path lengths.

The optical thickness of the sample is represented by the following equation:

$$
\mathrm{X}_{21}=\mathrm{X}_{2}-\mathrm{X}_{1}=\mathrm{n}_{\mathrm{g}} \mathrm{L}^{\prime}
$$

Where $L^{\prime}$ and $n_{g}$ are the physical thickness and refractive index of the sample. The apparent shift in the back reference reflector is represented by $X_{43}=X_{4}-X_{3}=\left(n_{g}-1\right) L^{\prime}$. Thus the refractive index of the sample medium can be found by the following equation[176]

$$
\mathrm{n}_{\mathrm{g}}=\frac{\mathrm{x}_{21}}{\mathrm{x}_{21}-\mathrm{x}_{43}}
$$

Once the group index is known, the physical thickness of the sample can be calculated using following equation:

$$
\mathrm{L}^{\prime}=\frac{\mathrm{x}_{21}}{\mathrm{n}_{\mathrm{g}}}
$$




\subsubsection{Results and discussions}

Figures 6.3(a) represents the cross-sectional image of the flow cell before inoculation. The two thick lines observed at the top of the image represent the reflections from the cover glass slip. The bacterial biofilms were cultured at the space between cover glass and the bottom surface of the channel. Figures 6.3(b) and 6.3(c) represent the cross-sectional images of PA-1 biofilm that are acquired on week 1 and week 2, respectively. Figure 6.3 (d) illustrates an A-scan signal from an empty flow cell, where the reflection peaks correspond to different layers in the empty flow cell. The first two peaks represent the reflections from the top and bottom surface of the cover glass.
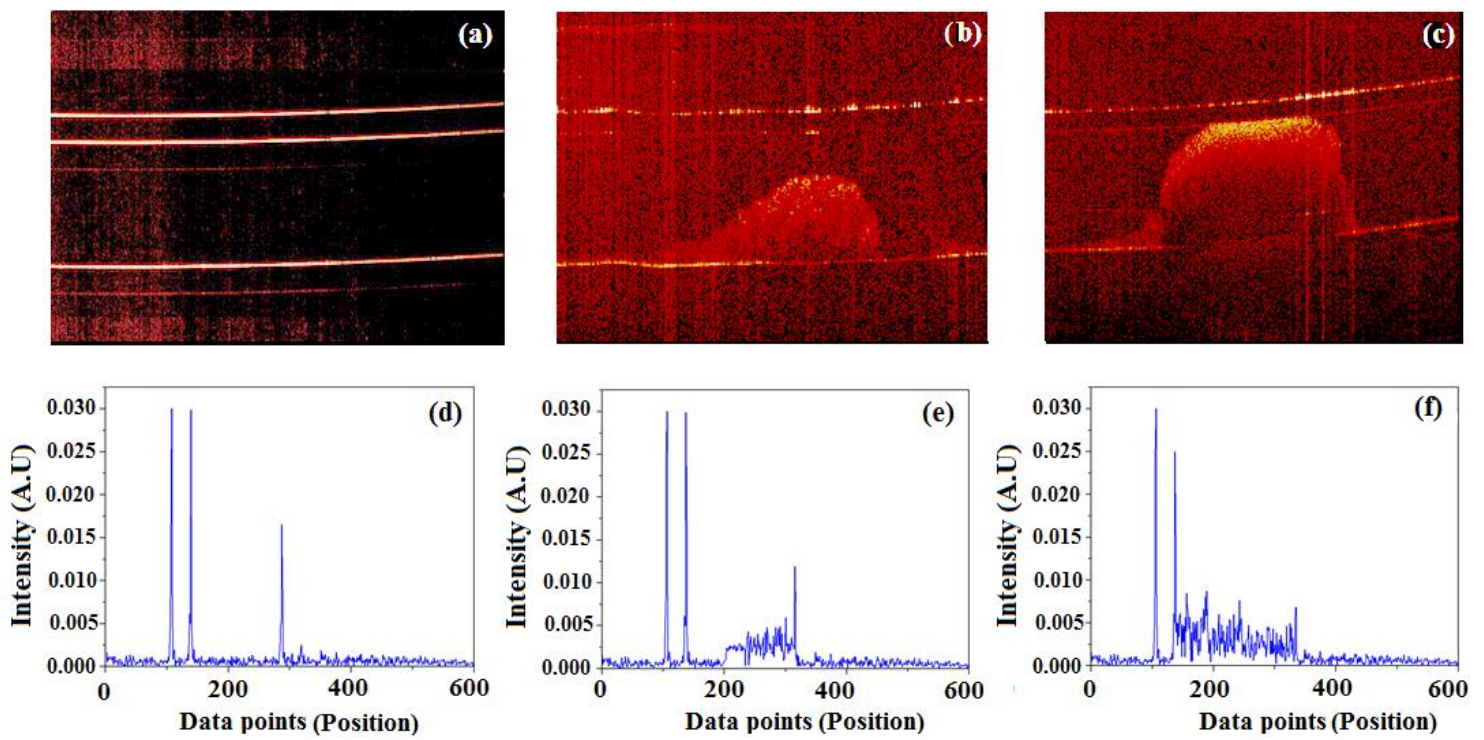

Figure 6.3 (a), (b) and (c) represent the cross-sectional images of an empty flow cell, flow cell with 1 week old biofilm and flow cell with 2 weeks old biofilm respectively;(d), (e) and (f) represent the A-scan signal from an empty flow cell, flow cell with 1 week old biofilm and flow cell with 2 weeks old biofilm, respectively.

The third peak represents the reflection from the bottom surface of the channel. In this experiment, the cover glass slip and the bottom layer of the flow channel are used as the reference surfaces for the measurement. The bottom surface of the flow cell can function 
as the back reference surface which eliminates the need of an additional (external) reference mirror or glass plate. The presence of the biofilms in the channel alters the optical path length inside the channel and the relative position of the reflection peak (or PSF) from the bottom surface is shifted. The position of the base layer of the channel with and without the biofilm is demonstrated in Figure 6.3(d), 6.3(e) and 6.3(f). Figure 6.3 (e) represents the A-scan signal from the biofilm measured at week 1. From the A-scan data shown in Figure 6.3(e), the optical path length of the biofilm is found to be $814 \pm 15 \mu \mathrm{m}$ (corresponding to 114 data points) and the relative shift in the position of the rear reflector is $214 \pm 4 \mu \mathrm{m}$ (corresponding to 30 data points). Using Equation (6.2) the refractive index value of the biofilm is found to be $1.356 \pm 0.022$. This gives a physical thickness value of $600 \pm 10 \mu \mathrm{m}$ based on Equation (6.3). Similarly, Figure 6.3(f) represents the A-scan signal from biofilm measured at week 2 . The optical path length of the film was measured to be $1.36 \pm 0.02 \mathrm{~mm}$ and the apparent shift in the location of the channel base is found to be $357 \pm 7 \mu \mathrm{m}$. This gives a refractive index value of $1.355 \pm 0.023$ according to the Equation (6.2). In this case, the thickness of the film is found to be $1.00 \pm 0.01 \mathrm{~mm}$, based on the Equation (6.3) which agrees well with the known physical dimension of the flow channel. From the above observations it is evident that the refractive index of the biofilm is closer to the refractive index of the water $(n=1.333)$, and in good agreement with previously reported values [47].

\subsection{Real-time in-situ investigation of Klebsiella Pneumonia using SSOCM}

The studies based on the tomographic images are useful only if the biofilms have sufficient thickness and structural features that are resolvable by the axial resolution of OCT system. The limited axial resolution of the practical OCT systems especially those operating at 
$1300 \mathrm{~nm}$ window, often prevents the investigation of the microbial growth or biofilm formation at the earlier stages of their development. This situation is more relevant in the case of biofilms such as Klebsiella Pneumonia which are generally developed in flat shape and without any distinct structures $[177,178]$.

The performance of the OCT can be improved to high lateral resolution via optical coherence microscopy (OCM), which combines the coherent detection with confocal microscopy. OCM can facilitate enface visualization with high lateral resolution sufficient to resolve both structural and cellular morphology without contrast agents. Therefore, OCM can provide better visualization of the development of the microcolonies over large field of view. OCM provides the enhanced penetration depth compared to the conventional confocal microscopy and improves the image contrast by rejecting unwanted scattered light from the out-of-focus region using coherent gated detection. Moreover, OCM based on Fourier-domain detection scheme allows simultaneous acquisition of the signals from different depths, thereby enabling the acquisition of enface image at any depth position. Furthermore, imaging at longer wavelengths, such as $1300 \mathrm{~nm}$, offers high quality images with improved penetration depth in turbid media.

\subsubsection{Materials and Methods}

\subsubsection{Biofilm growth}

Wild type Klebsiella Pneumonia, an environmental isolate, was maintained at $-80{ }^{\circ} \mathrm{C}$. The bacteria was cultured in either M9 minimal medium $\left(48 \mathrm{mM} \mathrm{Na}_{2} \mathrm{HPO}_{4} ; 22 \mathrm{mM} \mathrm{KH}_{2} \mathrm{PO}_{4}\right.$; $9 \mathrm{mM} \mathrm{NaCl} ; 19 \mathrm{mM} \mathrm{NH}_{4} \mathrm{Cl}$ ) supplemented with $2 \mathrm{mM} \mathrm{MgSO}_{4}, 0.1 \mathrm{mM} \mathrm{CaCl}_{2}, 0.04 \%$ w/v glucose and $0.2 \% \mathrm{w} / \mathrm{v}$ casamino acids (Supplemented M9) or Luria Bertani broth (LB10) $\left(10 \mathrm{gl}^{-1} \mathrm{NaCl} ; 10 \mathrm{gl}^{-1}\right.$ tryptone $5 \mathrm{gl}^{-1}$ yeast extract). 
The continuous flow-cell experiment was adapted from Reference [177, 179]. Biofilms were cultivated at $25 \pm 0.1{ }^{\circ} \mathrm{C}$ in three-channel flow cells with channel dimensions of $1 \times 4 \times 40 \mathrm{~mm}^{3}$ (IBI SCIENTIFIC). The flow-cells were connected to syringes and waste collection bottle with oxygen-permeable silicon tubing. The flow cells were supplied (via syringe pumps) with supplemented M9 minimal medium at $9 \mathrm{ml}^{-1}$ (mean velocity of $0.625 \mathrm{mms}^{-1}$ and associated Reynolds number of 1.12).

Overnight cultures of KP-1 were grown in LB10 medium from frozen stock and then streaked onto LB10-Agar. The streaked plates were incubated at $30^{\circ} \mathrm{C}$ for $24-\mathrm{h}$ and then refrigerated at $3^{\circ} \mathrm{C}$ for a maximum of 1 week. A single colony was selected and grown overnight in supplemented M9. Afterwards, the bacteria were pelleted, followed by resuspension in fresh supplemented M9 and incubation at $25^{\circ} \mathrm{C}$ for $1 \mathrm{~h}$.

Each channel of the flow-cell was inoculated with overnight culture, diluted to an optical density $\left(\mathrm{OD}_{600}\right)$ of 0.100 (UV-1800, Shimadzu, Japan), containing approximately $1 \times 10^{8}$ cfu $\mathrm{ml}^{-1}$. Incubation was made without flow, at $25^{\circ} \mathrm{C}$ for $1 \mathrm{~h}$ to allow cell attachment.

Three-day-old biofilms were treated with $0.1 \%$ w/v Sodium Dodecyl Sulfate (SDS) in supplemented M9 at a flow rate of $9 \mathrm{ml}^{-1}$ for $2 \mathrm{~h}$ to impose a stress on an otherwise flat biofilm with no distinct features. Biofilm growth was monitored by periodic measurements of the optical density $\left(\mathrm{OD}_{600}\right)$ and colony forming units of the effluent. It has already been established, in a related experiment that $\mathrm{OD}_{600}$ measurements of the effluent of the flowcell closely correlate with biomass measurements made by quantitative confocal microscopy image analysis using IMARIS and 16S rRNAs sequencing [177]. 


\subsubsection{Optical coherence microscopy imaging}

The experimental setup used for the real-time monitoring of the KP-1 biofilms is shown in Figure 6.4. The OCM probe was placed in the same incubator as the planar flow-cell and aligned with the top glass surface as shown in Figure 6.4 (a). The selective photographs of the flow-cell experiment and real-time monitoring using OCM setup are given in the Appendix D. The growth rate of KP-1 has been shown to be sensitive to glucose concentrations and as such, its biomass varies from channel inlet to outlet. For consistency, only images stacks of biofilms grown $5 \mathrm{~mm}$ from the inlet ends were used in this study. The light in the sample arm is collimated and focused onto the sample surface by a commercially available microscope objective with long working distance and $20 \mathrm{X}$ magnification (20X/air, LD Plan-Neofluar 441340, Zeiss, USA). The lateral resolution of the imaging system is quantified using an USAF resolution chart after immersed into growth medium at $1 \mathrm{~mm}$ depth and by holding a cover glass slip on the surface of the water. This is to replicate experimental conditions where the probe beam has to pass through cover glass slip and growth medium for imaging biofilms at the bottom surface of the flow-cell. Figure 6.4 (b) shows the enface image of the USAF chart. From the image, it is evident that smallest element that can be resolved is Group7-Element 6, which indicate a lateral resolution of $2.19 \mu \mathrm{m}$. This resolution is sufficient to resolve the microcolonies of the KP-1. The position of the beam waist is manually calibrated such that focal plane coincides with the bottom surface of the flow-cell. The laser beam was scanned over the flow cell channel in the $\mathrm{x}$ and $\mathrm{y}$-directions using two orthogonally aligned galvo mirrors (GVSM002, Thorlabs Inc., USA), $5 \mathrm{~mm}$ away from the flow cell inlet. The images are acquired at speed of 15 frames per second. This results in an image volume of voxel size $500 \times 460 \times 1040$, obtained after combining the individual B-scans. Enface images 
representing the bottom surface, with a dimension of $500 \times 460$ are extracted from the 3D volume data. This is corresponding to a field of view of $1.150 \mathrm{~mm} \times 1.030 \mathrm{~mm}$. Therefore, the developed OCM system provides the combined spatial and temporal resolution necessary for assessing the acute biofilms to consistent growth and perturbations.
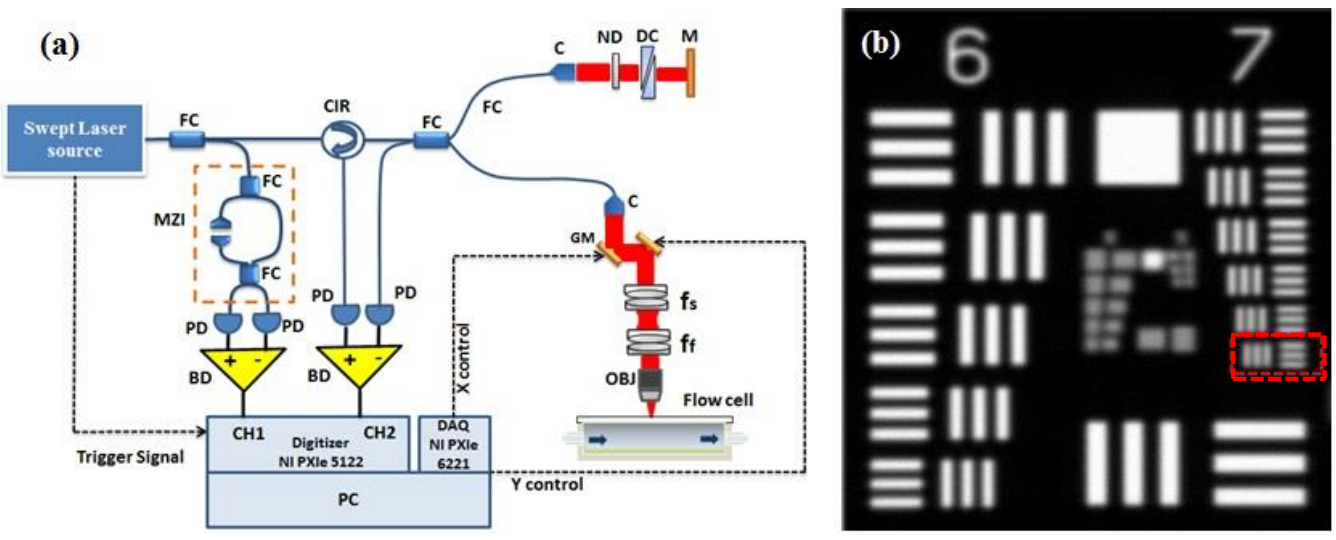

Figure 6.4 (a) Schematic of the planar flow cell configuration used for the biofilm growth and OCM imaging. (b) Enface image of the USAF chart demonstrating the lateral resolution of the imaging system. BD: Balance detector; C: Collimator; DAQ: Data acquisition and Control; FC: Fiber coupler; GM: Galvo Mirror; ILA: In-line variable Attenuator; PC: Personal Computer; PD: Photodetector. FS: scan lens; FF: tube lens.

\subsubsection{Real-time optical density measurement using OCM technique}

The quantitative analysis of the microbial growth is performed in terms of optical density (OD) of the bacterial microcolonies since they correlate directly with the cell concentration and biomass. Considering the single scattering model for the photon diffusion, the depthdependent decay of the OCM signal follows Beer-Lamberts law as [180, 181]

$$
I(z) \propto \exp (O D)
$$

where $\mathrm{OD}=\mu \mathrm{cL} ; \mu=$ extinction coefficient, $\mathrm{c}=$ concentration and $\mathrm{L}=$ optical path length of the medium. Since the extinction coefficient (for a single bacterial species) and the length of the channel are constant, then the OD directly correlates with the concentration of the 
biofilm. Besides providing morphological images, OCT can perform quantitative measurements of local optical intensities which would enable the estimation of optical density or reflectively of biological specimen [182]. The bottom surface of the flow cell can serve as reference surface, whose relative position and reflectivity can be used for the estimation of the refractive index and thickness of the biofilms as discussed in section 6.2.2. In the present experiment, the optical density is altered by the varying concentration of bacterial cells/biofilm leading to a subdued intensity from the bottom surface, according to the relation [182]

$$
R_{\text {new }}(t)=R_{\text {Ref }}-\int_{0}^{L} \mu c(t) d l
$$

where $R_{\text {Ref }}$ is the reflection peak corresponds to the base of the flow channel before inoculation, $R_{\text {new }}(t)$ is the reflection peak at any time instant $t$ and represents the time varying concentration of microbial which are dispersed in the medium as well as on the bottom surface. Therefore the optical density of the biofilms can be calculated by the relation

$$
O D(t)=R_{R e f}-R_{n e w}(t)
$$

\subsubsection{Measurement of planar density measurement}

A quantitative analysis of the biofilm growth is performed in terms of planar density which represents the ratio of area occupied by the microbial communities to the total field of view. The area occupied by the microbial communities is calculated using ImageJ, an image processing package that is available as freeware (http://rsb.info.nih.gov/ij/). 


\subsection{Results and discussions}

To facilitate the grown of biofilms on common engineering plastics encountered in real life situations, a standard planar flow cell was used. Figure 6.5 represents the enface image of the base of the flow cell channel. The surface roughness, scratches and holes are apparent from the topography of the lower plastic base. A side-by-side comparison is made by bright field microscopy (Nikon Eclipse LV100 with 20X objective) and demonstrates that these features are not artifacts induced by OCM.
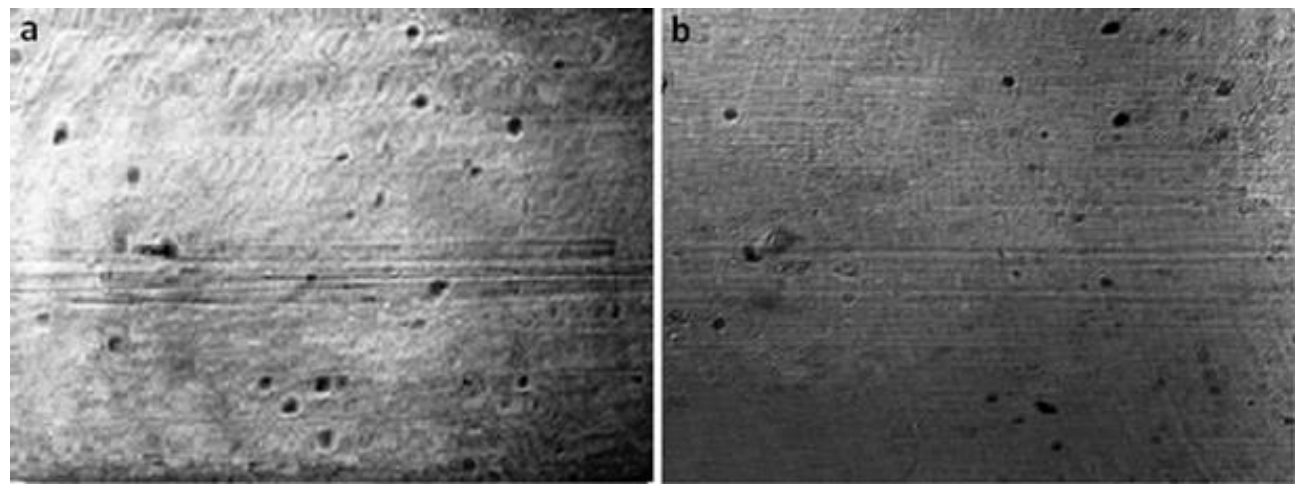

Figure 6. 5 (a) Enface image of bottom surface of the flow cell channel (a) acquired by OCM (b) acquired by light microscopy. The image sizes are $1.3 \mathrm{~mm} \times 1.0 \mathrm{~mm}$.

Figures 6.6 (a)-(f) show the representative images (Day 1, Day 3, and Day 3 just 2 hours after perturbation) of the growth dynamics of a KP-1 biofilm as imaged continuously over a period of 6 days. The non-uniform profile of the base surface reduces the visibility of microcolonies. In order to study the spatial localization of microcolonies and their quantification, image processing methods have been adopted. The enface images with microcolonies are subtracted from the background image shown in Figures 6.5 (a), followed by edge detection using ImageJ. The resulting images highlights the regions 
occupied by the bacterial microcolonies and are illustrated in Figures 6.6 (b), 6.6 (d) and 6.6 (f), respectively.

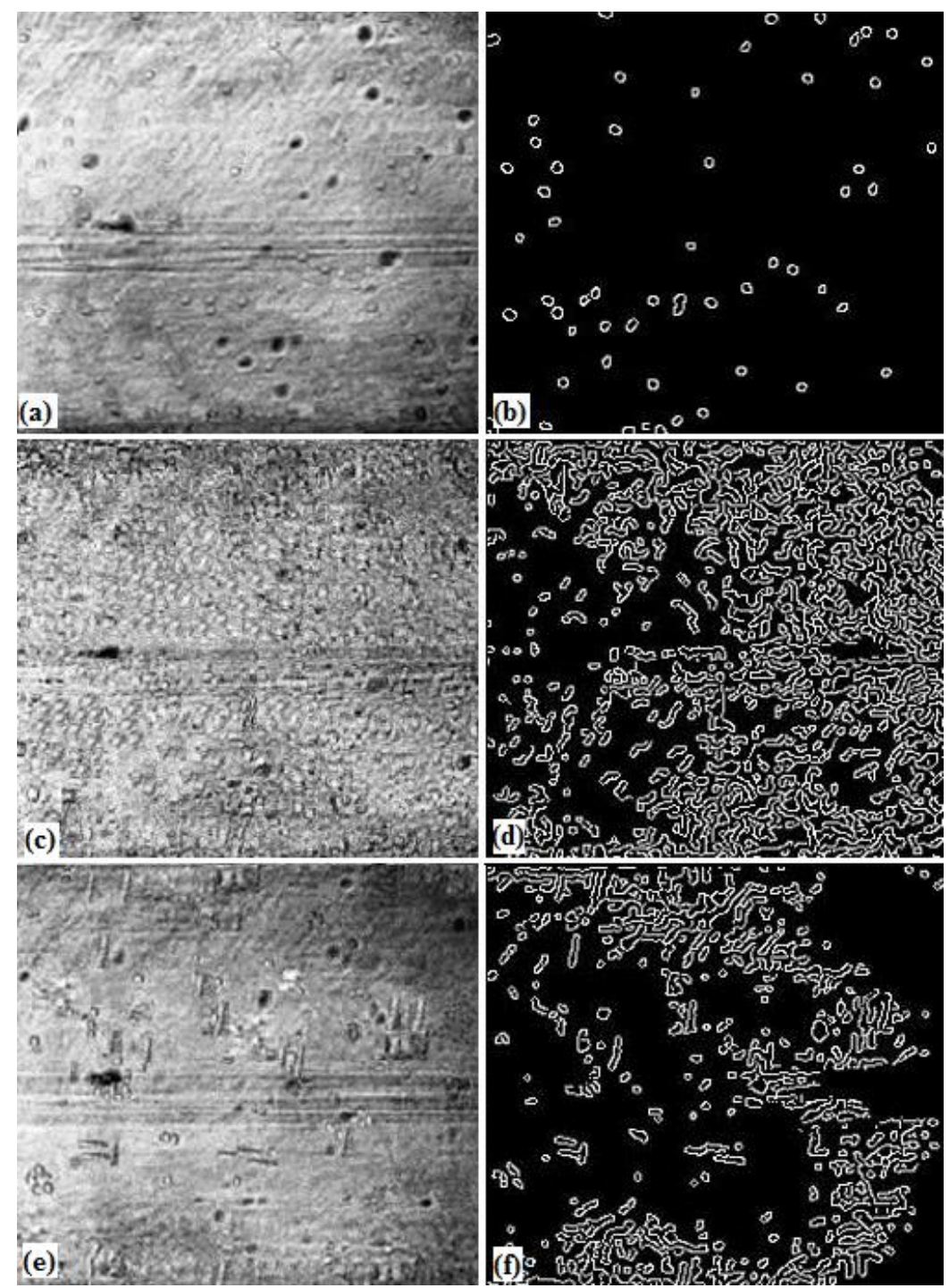

Figure 6.6 Enface images and edge detected images of the $K P-1$ microcolonies formed on the base of flow cell channel. Images (a), (c) and (e) correspond to biofilm growth on day 1, day 3, and on day 3; $2 \mathrm{~h}$ after perturbation. Images (b), (d) and (f) corresponding edge detected images. The image sizes are $1.150 \mathrm{~mm} \times 1.030 \mathrm{~mm}$.

On day-1, fragmented biofilm regions are apparent (see Figures 6.6 (a)). By day-3, a mature KP-1 biofilm is visible and occupies most of the base area, as shown in Figures 6.6 
(b). On day-3, the matured biofilm is treated with $0.1 \%$ of w/v sodium dodecyl sulfate (SDS), and the recovery from the transient response was observed for the next three days (day-4 to day-6). Figures 6.6 (e) represents the image acquired $2 \mathrm{~h}$ after the perturbation, demonstrating significant reduction of the biomass in response to the perturbation. The biomass has reached its minimum level on day-4, and gradually recovered over the following days (day-5 and day-6). The complete set of enface images of microcolonies of KP-1, and their background removed and edge detected images are given in the appendix E. This result is consistent with the growth rate reported for the same KP-1 biofilm, with identical experimental conditions [177].
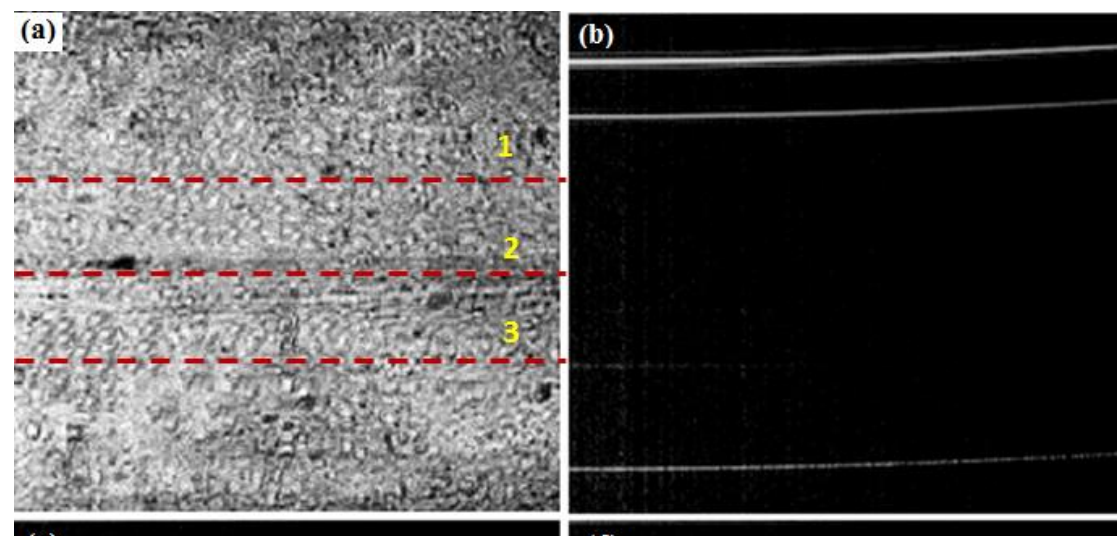

(c)

(d)
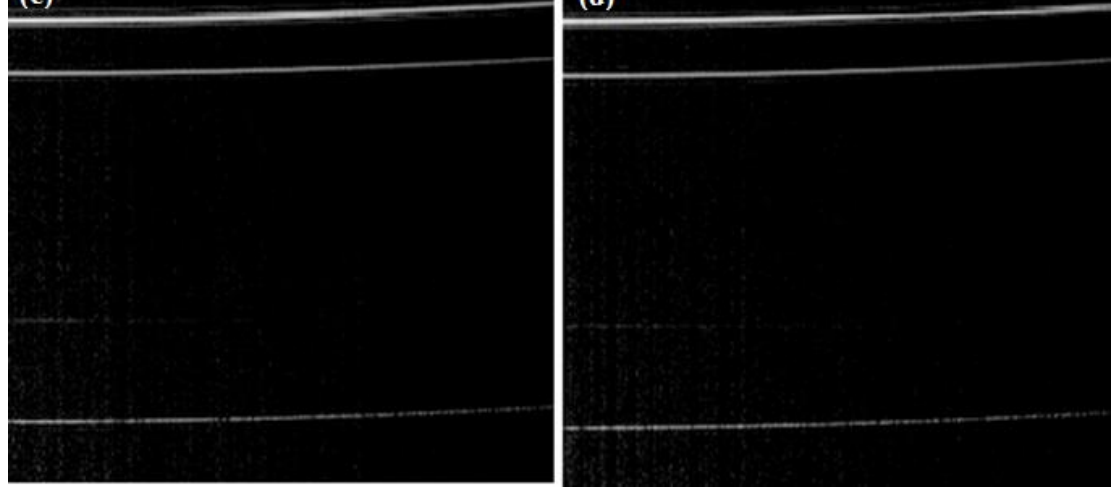

Figure 6.7 Enface image of KP-1 biofilm on matured state (Day-3). (b), (c) and (d) shows the cross sectional images acquired from the vertical positions indicated by the red dotted lines and numbers respectively. 
The cross-sectional images of the flow cell (on day-3) are provided in the Figure 6.7. The figures illustrates that the biofilms developed at the bottom cannot be resolved by the axial resolution of the system. On the other hand, the enface images with high lateral resolution provides the better visualization of bacterial microcolonies and their development at the bottom surface of the flow cell.

Figure 6.8 demonstrates the representative averaged A-scan profiles acquired from the volume under investigation before and after the biofilm growth. The first two peaks in the profile represent the reflections from the top and bottom surfaces of the glass cover slip. The third peak represents the reflection from the base of the flow cell channel. The region between the top and bottom reflections represents the growth medium, where the signal attenuation is dominant due to the absorption. It is known that the plankton cells and biofilms that are present in the flow-cell will alter the optical density. The variation in the OD would be reflected as a variation in peak reflection from the base surface.

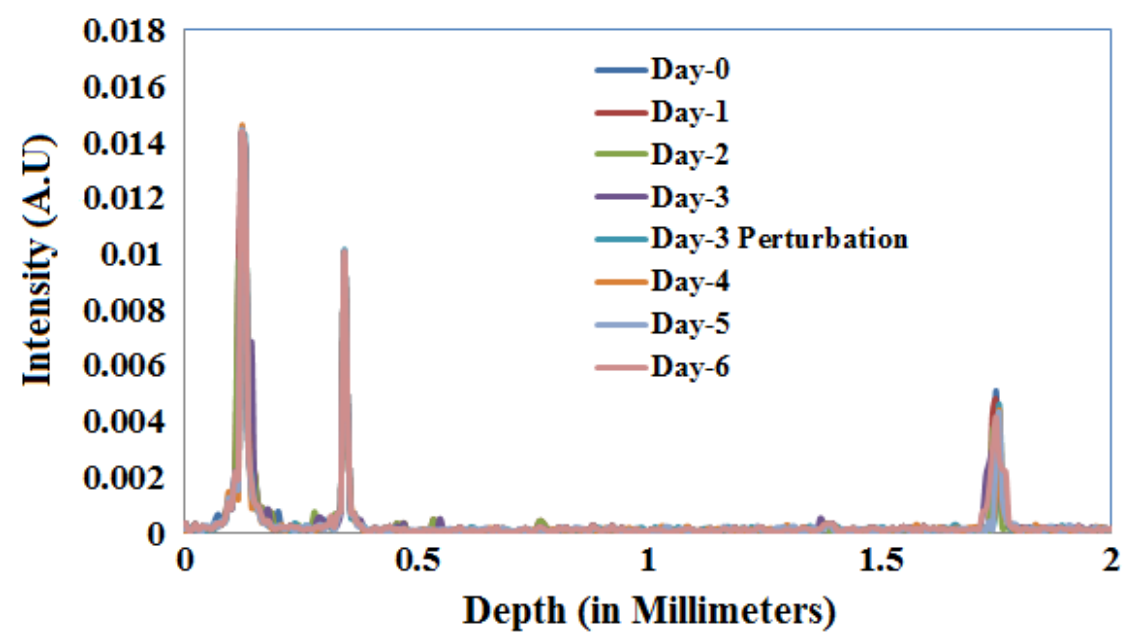

Figure 6.8 A-scan profiles (averaged) demonstrating a reduction of the reflection peak caused by the presence of the microbial. 
Figure 6.9(a) represents the optical density (OD) plot obtained by the standard approach based on UV-VIS spectroscopy $\left(\mathrm{OD}_{600}\right)$ of the effluent. Similarly, colony forming unit (CFU) measurements are illustrated in Figure 6.9(b). These measurements are different from OD since they only quantify the viable cells that are able to be cultured into colonies. Nevertheless, the trend in CFU's is similar to those for OD. Figure 6.9(c) represents the OD plot obtained using OCM techniques based on the Equation (6.6). It is observed that OD measured using the OCM technique is consistent with the OD measured using spectroscopy. However their absolute values differ due to the usage of different wavelength regimes for their measurement. It can be seen that the OD increases from day1 to day-3 representing the growth of biofilm. On day-3 OD reaches a maximum indicating that the biofilm is now mature. The reduction of the OD can be observed on day- 3 to day4, representing the reduction of biomass in response to the perturbation by SDS. The recovery of the biofilm on days 5 and 6 was also exhibited by the OD analysis as a gradual increase in the OD values. Quantitative analysis of biofilms based on the OCM enface images were also made. Figure 6.9(d) represents the planar density of the biofilm microcolonies, which represents the ratio of area occupied by the microbial communities to the total field of view. Figure 6.9(d) represents the variation of the planar density over different days. It is evident from the illustrated figures and analysis that the quantitative assessment of the biofilm growth using both OCM technique and standard procedures are closely agree with the enface images based analysis. This demonstrates the potential of optical coherence microscopy for quantitative in-situ monitoring of microorganisms and their clusters such as bacterial biofilms. 

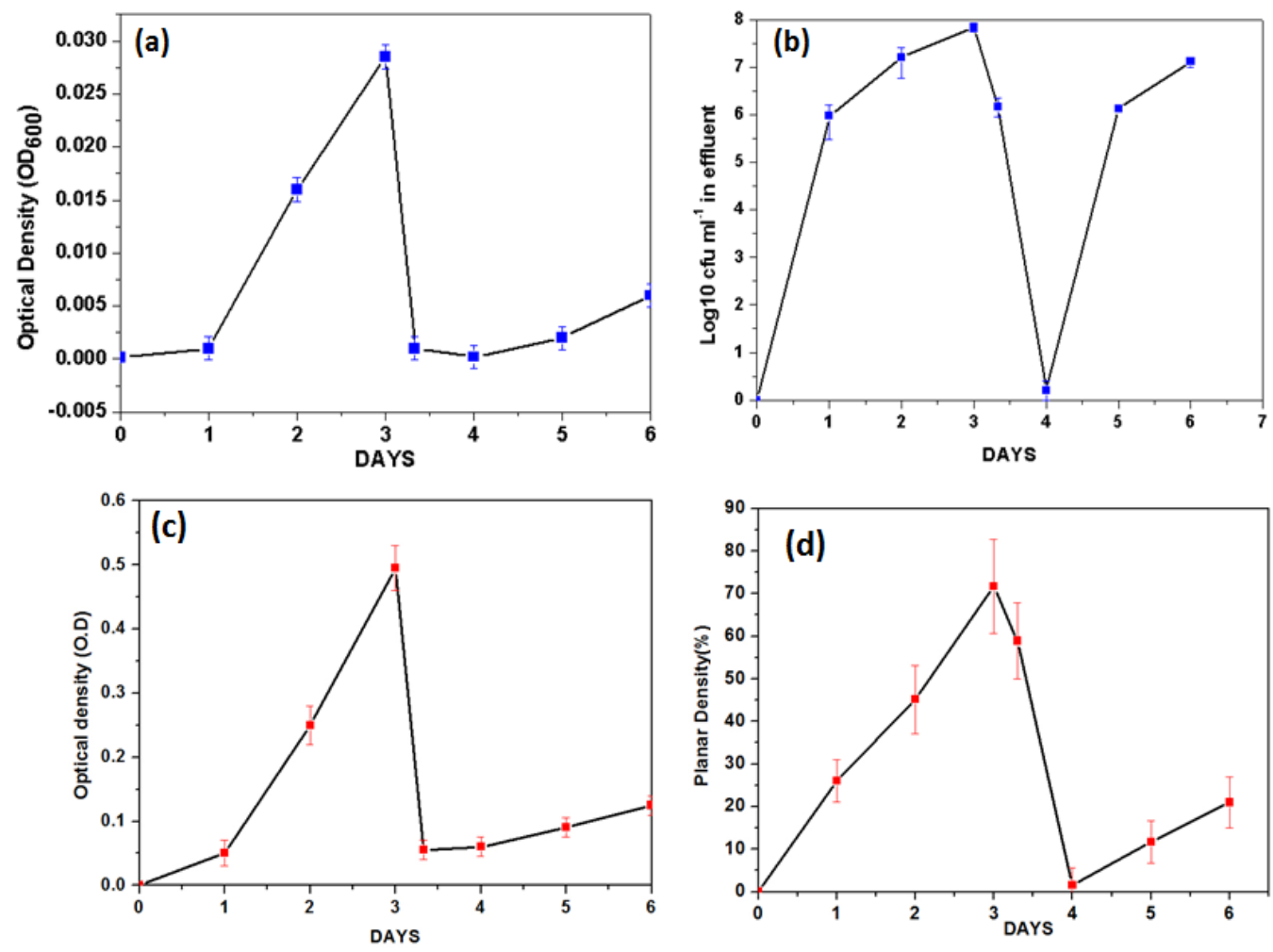

Figure 6. 9 (a) Standard Optical Density $\left(\mathrm{OD}_{600}\right)$ measurements and (b) Colony Forming Unit (CFU) measurements made by periodically sampling the flow-cell effluent. (c) Optical density measured via OCM and (d) planar density measurements of the biofilm via OCM

\subsection{Conclusion}

The potential of optical coherence imaging for real-time in-situ monitoring of the bacterial biofilms is demonstrated. The experimental investigations are performed using a flow cellbased biofilm culture setup due to its similarity with the practical scenarios in which biofilms are developed. The development of Pseudomonas Aeruginosa (PA-1) biofilms is monitored by acquiring the cross-sectional images of biofilms. The cross-section images of one week and two week old PA-1 biofilms were acquired at an axial resolution of $8 \mu \mathrm{m}$ and lateral resolution of $18 \mu \mathrm{m}$. A novel methodology for simultaneous measurement of refractive index and the thickness of bacterial biofilms was demonstrated. The apparent shift in the reflection peak caused by the biofilm growth in the flow channels was used to 
determine the group index and the thickness of the biofilm. The measured refractive index of PA-1 was found to be close to that of water. It is observed that the refractive index calculated by the proposed method is in good agreement with the previously reported value. The proposed method is very simple to implement and can be used for precise measurement of thin films and transparent materials.

The potential of the optical coherence microscopy for real time in-situ investigation of the thin biofilms was further explored. The development of the Klebsiella Pneumoniae (KP-1) biofilms and the transient processes in response to the antibacterial treatment was demonstrated using enface images. Enface images of the microcolonies developed at the bottom surface of the flow cell channel were acquired using a swept-source based optical coherence microscopy system (SSOCM). All the enface images were acquired at a spatial resolution of $2.2 \mu \mathrm{m}$. The growth of biofilms were quantified in terms of optical density and planar density using OCM technique; which was found to be consistent with the assessment based on the spectroscopy based OD values and colony forming units (CFU). It is envisaged that this in-situ monitoring with OCM can make impactful contributions towards various biofilm analysis and their growth monitoring in-vivo. 


\section{Chapter 7: Conclusions and Future Research Directions}

This chapter begins with the brief descriptions on the outcomes of the various investigations conducted, followed by highlighting the salient and original contributions made. The future research directions are explained by highlighting the potential research challenges ahead.

\subsection{Conclusions}

A compact swept source-based optical coherence tomography system with enhanced imaging capabilities has been conceptualized, established and experimentally demonstrated. An interferometry system based on the non-reflective reference arm has been developed and incorporated in the SSOCT system. The proposed configuration avoids the need of bulky and alignment sensitive mirror based reference arm and provides a highly stable and compact configuration for the SSOCT system. The imaging was performed at a wavelength of $1320 \mathrm{~nm}$ in order to attain improved penetration depth and reduced back scattering in highly turbid media. The imaging capabilities of the SSOCT system are improved by enhancing the axial resolution and sensitivity. The degradation of axial resolution and sensitivity caused by the non-linear sweeping characteristics of the wavelength swept-laser sources is efficiently corrected by a novel spectral-phase based calibration scheme. The proposed spectral phase-based scheme is performed automatically during the laser scanning, without relying on external hardware resources. It has shown that the proposed method outperforms the existing calibration scheme in terms of axial resolution, sensitivity roll-off and computational efficiency. Thus, the proposed method 
offers an accurate and cost effective solution for the calibration in SSOCT system without increasing computational and hardware complexity. The axial resolution and sensitivity of the system is further improved by correcting the optical dispersion using a numerical algorithm. It has shown that the dispersion can be efficiently corrected by eliminating the dispersion-induced spectral phase component from the OCT signal. The proposed method is shown to be computationally efficient and corrects the dispersion more accurately. The quality of image is further enhanced via suppressing the side lobes in the point spread functions. A spectral apodization method based on the Hamming window is shown to be efficient for suppressing the side lobes in the proposed system.

The developed system is theoretically and experimentally characterized using the key performance parameters such as spatial resolution, sensitivity roll-off, imaging speed and imaging depth. The lateral resolution of the system is experimentally measured using a resolution chart. The United States Air Force (USAF) chart based resolution characterization shows that the proposed system exhibits a lateral resolution of $17.5 \mu \mathrm{m}$. The axial resolution is determined by measuring the FWHM of the PSF. The measured axial resolution in air is found to be $8.3 \mu \mathrm{m}$, which is close to the theoretically predicted value. Moreover, the proposed SSOCT system maintains nearly constant axial resolution throughout the imaging depth. The experimental investigation shows that a sensitivity of $110 \mathrm{~dB}$ and a principal imaging range (-6 dB imaging depth) of $1.5 \mathrm{~mm}$ is achieved. It is worth to mention that all the experimentally determined imaging parameters are in good agreement with their theoretically predicted values. This shows the accuracy and efficiency of the proposed instrumentation and signal processing schemes. The enhanced imaging 
capability of the system is further illustrated using high quality cross-sectional images of highly scattering and dispersive media.

The plasmon resonant silica-coated silver nanoplates and gold nanorods with higher aspect ratio are shown to be powerful contrast agents for OCT imaging around $1300 \mathrm{~nm}$. The dimensions and the structure of the nanoparticles are chosen such that the surface plasmon resonance wavelength overlaps with the central wavelength of the light source. FDTD based simulation results show that SSNPs with selected dimension exhibits peak extinctions at $1320 \mathrm{~nm}$ and can function as an excellent scattering based contrast agent for OCT. It is shown that the extinction coefficient of highly scattering nanoparticles can be accurately determined from the A-scan profile using a calibration function. It is found that the extinction cross-section measured using OCT is reasonably consistent with the spectroscopy and FDTD simulation results. Experiments on pig adipose tissues show that LSPR induced scattering in SSNPs contributes to the OCT signal and offer a better image contrast. It is evident from the illustrated images and average A-scan signals that SSNPs can efficiently penetrate into tissue structures. The variation of speckle patterns with respect to the static morphological tissue structures can be efficiently used for tracking the nanoparticles. Accordingly, speckle variance OCT imaging is demonstrated as a powerful method for the analysis of distribution of the nanoparticles in tissues.

Gold nanorods (GNRs) with higher aspect-ratio are shown to be powerful photo-absorption agents at higher imaging window. The FDTD results show that the longitudinal plasmon wavelength of the GNR can be tuned to higher wavelength window by varying its aspect ratio. It is shown that, GNRs with aspect ratio 8.8 exhibits longitudinal peak at $1320 \mathrm{~nm}$. A cross-correlation method based on the OCT is used to measure the extinction coefficient of 
highly absorbing GNRs. The proposed method avoids the complex fitting process and calibration function for correcting the confocal effect of the imaging optics. The extinction cross-section measured using the proposed method is found to be in good agreement with the spectroscopy method and FDTD results. The cross-sectional images acquired from GNRs embedded agar-gel phantom shows that GNRs with the selected dimensions can act as excellent absorption based contrast agents for OCT imaging.

The potential of optical coherence imaging for real-time in-situ and quantitative monitoring of the biofilms is explored in this thesis. It has shown that OCT performs labelfree imaging and reveals the spatially resolved structures of biofilms. The cross-sectional imaging with OCT enabled the time resolved development of thick biofilms of Pseudonymous Aeruginosa (PA-1) in a flow cell. It has shown that the apparent shift in the position of reference peak caused by the biofilm growth can be used to determine the refractive index and thickness of the biofilms. The proposed method is simple and allows the measurement more precisely during the development of biofilm. The refractive index of the PA-1 biofilms is found to close to water and in good agreement with the previously reported value.

The optical coherence microscopy is demonstrated as a powerful tool for quantitative imaging of the development of microcolonies of bacteria and their spatial localization. The capability of SSOCM for volume imaging and acquisition of enface images is demonstrated. The time-resolved development of the Klebsiella Pneumonia (KP-1) microcolonies and transient process in response to the antibacterial treatment are investigated using enface images. It is shown that quantitative measurement of local optical intensities allows the real-time assessment of optical density of biofilms. The 
quantification of biofilms using planar density is also explored. It is evident from the illustrated figures that the assessment based on OCM agrees well with the standard spectroscopy based OD and colony formation unit (CFU) measurements. It is envisaged that this in-situ monitoring with OCM can make impactful contributions towards various biofilm analysis and their growth monitoring in-vivo. Moreover, this proposed methodology can be extended to the possible use of this bio-optic probe variety of different applications such as for the detection of the resistance to therapeutic agents during treatment of chronic infections in-vivo.

\subsection{Major Contributions}

The major contributions of this research thesis are summarized as follows

* A compact swept source based optical coherence tomography system is configured and developed.

* A real-time automatic calibration scheme for swept source based optical coherence tomography is established and demonstrated.

* Contrast enhancement in OCT using silica coated silver nanoplates and gold nanorods with long aspect ratio are demonstrated.

FDTD based simulation models with related methodologies and algorithms for the determination of optical properties of nanoprobes are proposed and experimentally demonstrated.

* The extinction cross-section of nanoparticles was calculated with theoretical formulations followed by experimental demonstration.

The capability of OCT for real-time in-situ imaging of time-resolved development of Pseudonymous Aeruginosa is demonstrated. 
A methodology for the real-time and simultaneous measurement of refractive index and physical thickness of biofilms (Pseudonymous Aeruginosa) is demonstrated.

* The potential of swept source optical coherence microscopy (SSOCM) for real-time in-situ and quantitative investigation of the development of bacterial microcolonies (Klebsiella Pneumonia) is demonstrated.

Real-time quantification of optical density and planar density of the biofilms is demonstrated.

\subsection{Future Works}

There exist numerous possibilities for future studies in optical coherence imaging in term of its instrumentation, imaging capability and applications. This section details a brief list of studies that naturally seems to be extensions of the research work described in this thesis.

* The compactness of the OCT system can be further improved by eliminating the complex galvo mirror based beam steering setup. The future research can be directed towards developing a compact spatial division multiplexing system that allows parallel illumination and acquisition of the optical beams from the sample. This approach totally avoids the use of additional DAQ card for control generation. The implementation of the proposed parallel acquisition system is illustrated in Figure 7.1. The proposed system consists of a 1xN PLC splitter with inbuilt delay for output channels. This is attached to a lens plate via an optical epoxy. The lens plate has multiple micro lenses inscribed on it forms a lens array. The light from the sample arm is spatially divided by the PLC and the light travel through the individual channel is distinguished via their time delays. The light arrives at the output facet of the PLC, travel through an optical epoxy $(\mathrm{n}=\sim 1.5)$ and then 
through the lens plate. The reflected light from the sample is collected back via the illumination lens. The light from the individual points are directed towards the interferometer through the PLC channels.

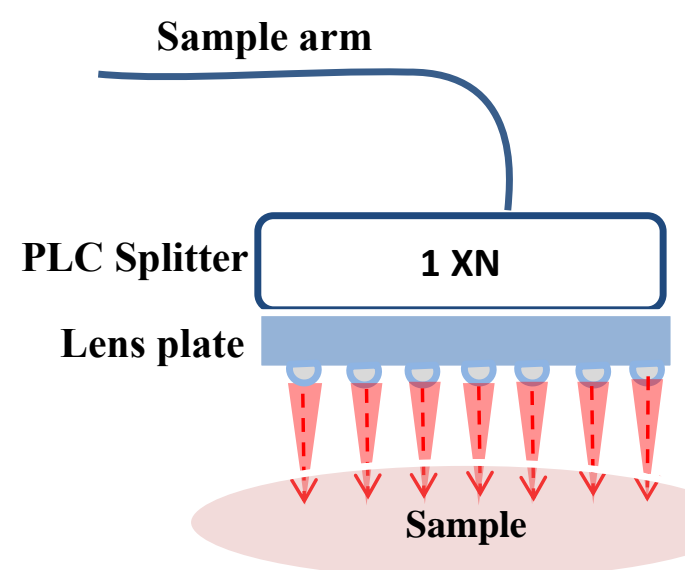

Figure 7.1 Schematic of the proposed beam delivery system via spatial multiplexing

* The application of the spatial multiplexing probe for complex imaging applications such as intracoronary and retinal imaging can be considered as another future work. For intracoronary applications, a circumferential scanning probe is generally used. A spatial multiplexing probe with lens array aligned over the circumference of catheter/ endoscopic probe can eliminate the rotation scanning system and tremendously increase the imaging speed.

* The high absorption-to-scattering cross-section ratio of the GNRs with higher aspect ratio predicts them as promising candidate for photothermal applications. Accordingly, future work can be directed towards the quantitative investigation of photothermal effects of the proposed GNRs and their applicability in photothermal therapy and surgery. Similarly, quantification of the nanoparticles in biological system is (i.e., cells, tissues and organs) is becoming a vital part of nanotoxicological and nanomedical fields. Therefore, the future work 
can be further focused on the quantitative approaches based on OCT to investigate biodistribution of nanoparticles in tissues and cell uptake.

* The existing capabilities of OCT have a great potential for noninvasive, label-free, realtime, in-situ and/or in-vivo investigation of biofilms and their characterization. Future research directions in biofilm investigation will be focusing on the functional extensions of the OCT to improve the characterization of biofilm structure and function. The biofilms are generally developed on the aquatic surfaces and their structure and functions will be greatly influenced by the flow dynamics. The potential of the OCT to perform real-time acquisition of velocity profiles in small lumens and vessels can be explored in this regard. Similarly, Doppler OCT imaging can provide quantitative information on the biofilm dynamics, flow velocities, and flow distributions during the development of biofilms.

* The biofilm structure and function is also influenced by the nature of the substrates and nutrient heterogeneity. Therefore, the future research can be focused on spectroscopic OCT (SOCT) for high-resolution spatial localization of spectroscopic information from a sample. The use of ultrahigh resolution OCT (UHR-OCT) will allow the differentiation cells and the polymer matrix in biofilms. Using appropriate thresholding techniques, the cells and polymer matrix in the biofilm can be differentiated. Accordingly, biomass can be calculated from each image. Similarly, Fourier domain OCT with high acquisition rate allows the imaging of bacterial growth in biofilms in 3-D volumes in real time. These quantitative data will be useful for mathematically modeling biofilm growth, as it will provide data of a complete biofilm rather than a portion of a biofilm. 


\section{APPENDICES}




\section{Appendix A: Configuration of swept-laser source}

Thorlabs' frequency swept tunable lasers are specifically designed for Swept Source Optical Coherence Tomography (SSOCT) and Optical Frequency Domain Reflectometry (OFDR) applications. These applications provide real-time, high resolution, cross-sectional imaging of turbid media and require a specially designed laser source that can sweep a wide wavelength range at very high speeds. A wide spectral tuning range is required for high axial resolution OCT images, and a high sweep-speed is needed to obtain real-time 2D and 3D OCT imaging speeds.

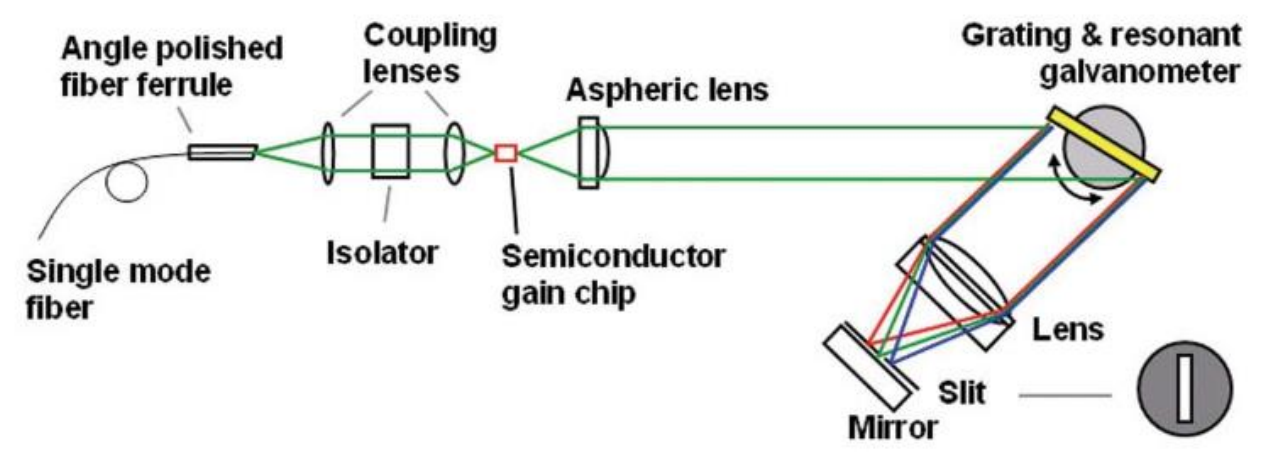

Figure A.1 Schematic of the swept source laser [183]

SL1325-P16 is based on external cavity laser geometry. This external cavity laser consists of a single gain element where one facet of the element serves as an end mirror for the cavity. The extended cavity is comprised of a single collimating lens and a Cat-Eye wavelength selection device [183]. The intra-cavity side of the semiconductor gain element is AR coated, providing a residual reflectivity of less than $10^{-4}$ thus allowing for the efficient formation of an extended cavity. Wavelength selection is achieved using a diffraction grating mounted onto a scanner with a focusing lens, mirror, and slit assembly 
providing active wavelength selection. The focusing lens and slit/mirror assembly are separated by the focal length of the lens. This configuration is commonly referred to as a Cat-Eye and is highly insensitive to angular misalignment. Output from the laser cavity is coupled into a fiber using a lens system containing an isolator that prevents optical feedback into the cavity. This design enables a robust alignment due to the cat's eye configuration of the back-reflector which provides superior long-term stability .compared to designs with a quasi-collimated beam on the laser cavity back-reflector. 


\section{Appendix B: Phantom preparation}

The study of photon migration in strongly scattering media is gathering increased interest due to the growing number of applications in medicine and biology, such as functional monitoring of blood oxygenation or glucose concentration, optical mammography, and in vivo measurement of the absorption spectrum of exogenous dyes [184, 185]. For the development and the proper assessment of a diagnostic or spectroscopic technique, a test on a tissue phantom is highly desirable. The phantom should mimic biological tissues and allow the easy tuning of the optical properties.

Over the last few decades, considerable efforts have been put to develop phantoms for optical imaging and spectroscopy. The diverse spectral and geometrical range of the optical applications has made the establishment of tissue phantoms arduous and fairly complicated. Most of the reported phantoms simulate optical properties at selected wavelength, are instable over time, or cannot be used to replicate stratified tissues of varying optical properties. In addition, optical phantom design is dependent upon the desired optical and physical properties for specific applications. Due to the increasing importance of precise fabrication of phantoms, a series of fabrication approaches have been experimented and only a few were generally accepted. The main constituents for sample fabrication include a host medium, includes the usage of epoxy resin, polyester or silicone as these materials provide enhanced shaping flexibility. The three main choices for scatters are white metal oxide powders $\left(\mathrm{Al}_{2} \mathrm{O}_{3}\right.$ or $\left.\mathrm{TiO}_{2}\right)$, lipid microparticles and polymer particles [186]. To introduce absorption properties into the host material, various kinds of dyes and phospholid emulsions have been investigated [187]. 
We preferred to use agar instead, since it allows a higher hardening of the sample. Moreover, highly purified agar powders are available with almost no absorption and very low turbidity. Finally, these products are well characterized and more controlled, since they are in use for standard biological routines.

\section{Materials and method}

The main constituents for fabricating an optical phantom include a host medium, absorbing agents and scattering agents. The following section describes the various materials used for phantom preparation.

\section{Host medium}

Various types of materials have been used as host medium to house scatters and absorbers. These materials fall principally under three categories such as solid, aqueous suspension or liquid and hydrogels $[185,186]$. However, the characteristics such as stability, refractive index, cost and ease of fabrication and biological compatibility varies across the choice of materials and should be carefully considered for its usage in the desired investigation. Recently agar is highly preferred as host medium for making phantom due to the negligible absorption and very low turbidity. Thus, the desired optical properties of the phantom are reached by adding appropriate amounts of $\mathrm{TiO}_{2}$ (Pharmacia, Italy), as a scattering medium. Finally, these products are well characterized and more controlled, since they are in use for standard biological routines 


\section{Scattering agent}

The choice of the scattering agent hinges upon considerations such as the type of embedding base material, its limitations and stability issues. $\mathrm{TiO}_{2}$ nanoparticles show excellent scattering properties at wide range of optical spectrum and their scattering properties can be easily predicted using Mie theory. Moreover, $\mathrm{TiO}_{2}$ allows the linear adjustment of scattering properties via changing its concentration.

\section{Fabrication methodology}

A highly purified agar powder (A-7049, Sigma-Aldrich, USA) of $100 \mathrm{gm}$ is dissolved in $10 \mathrm{ml}$ distilled water is used as the host medium. The agar solution alone has negligible absorption and very low turbidity. Thus, the desired scattering properties of the phantom are reached by adding appropriate amounts of $\mathrm{TiO}_{2}$ nanoparticles. $10 \mathrm{mg}$ of $\mathrm{TiO}_{2}$ is added to agar solution and sonicated for 10 minutes in order to disperse $\mathrm{TiO}_{2}$ uniformly throughout the solution. Without sonication, the particle settling can cause some unwanted spatial variations. After the sonication, the solution is heated up using a hot plate at $70^{\circ} \mathrm{C}$ for 10 minutes. At $70^{\circ} \mathrm{C}$ the solution is poured into the mould and left there for some time to reach a proper hardening and stable optical properties. Rapid cooling, as obtained by immersion in cold water, can be suggested, but freezing should be avoided, since it can change the optical properties. As typical examples, if immersed in cold water (at $10^{\circ} \mathrm{C}$ ), a 1 $\mathrm{cm} \times 1 \mathrm{~cm} \times 10 \mathrm{~cm}$ sample will require less than $5 \mathrm{~min}$, while a $15 \mathrm{~cm} \times 15 \mathrm{~cm} \times 5 \mathrm{~cm}$ block 3-4 h. 


\section{Appendix C: Qualitative Investigation of photothermal effect of gold nanorods}

Although, the photothermal effect caused by the LSPR-induced optical absorption in GNRs has been widely observed, measurement of local temperature variations in biological samples is still difficult. The photothermal effect generated by the LSPR of GNRs is demonstrated by delivering the GNRs into a pig adipose tissue sample and followed by the continuous scanning of OCT. The typical size of the pig adipose cells ranges from 100 to $200 \mu \mathrm{m}[166,187]$. These cells are generally opaque to the NIR wavelength at a temperature below $25^{\circ} \mathrm{C}[166,187]$. However, these cells become transparent and visible at a higher temperature. This property of the pig adipose cells is used herein to demonstrate photothermal effect. The adipose tissue is refrigerated before the OCT scanning. Figure C.1 (a) shows the cross sectional images of fresh pig adipose tissue just after refrigeration. Since the temperature is very low, the cells in the adipose tissue became opaque. The tissue sample was removed from refrigerator and it took around three hours to observe transparent cells at an ambient temperature of $27^{\circ} \mathrm{C}$. Figure C.1(b) represents the OCT image of the adipose tissue, after keeping at a normal temperature for three hours. In order to study the local heating effect, the droplet of GNRs was delivered onto the refrigerated adipose sample followed by a continuous scanning by OCT. Figure C.1 (c) shows the GNR droplet deposited over the sample surface. The droplet appears dark due to a strong absorption by the plasmon-resonant GNRs. The lower physical size of GNRs allows them to easily penetrate into the deeper regions of the tissue and occupy the intercellular substance. During OCT scanning, the LSPR induced absorption causes local heating of the surrounding tissues. This causes a thermo-elastic expansion in the 
microenvironment surrounding the absorber, resulting in visibility of the adipose cells as shown in Figure C.1 (d).

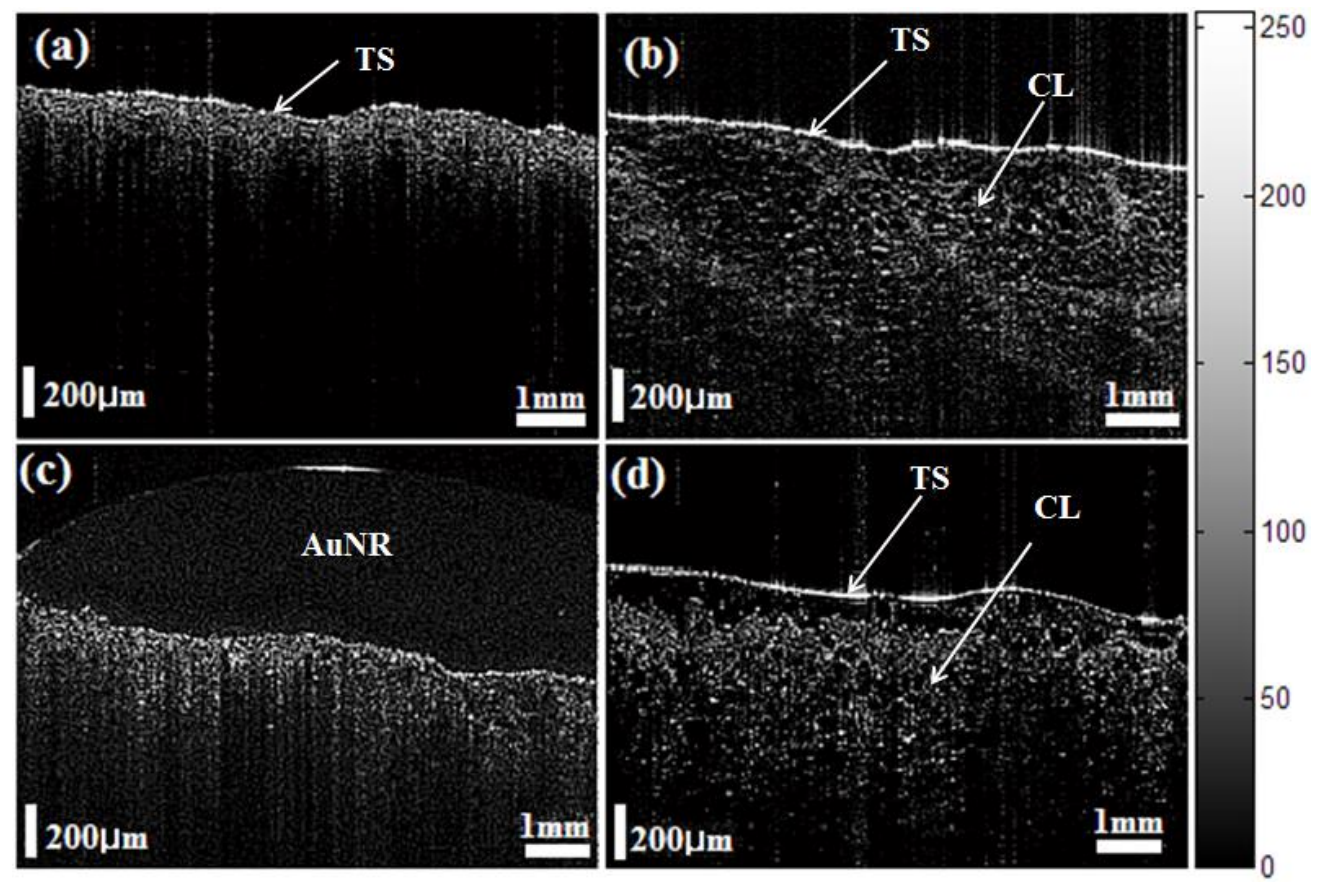

Figure C.1 OCT images of pig adipose tissue (a) Just after refrigeration (b) After three hours at normal temperature (c) GNRs droplet deposited over tissue sample just after refrigeration (d) 15 minutes after the GNR deposition. TS: tissue surface; CL: adipose cells 


\section{Appendix D: Experimental arrangement for the real- time in-situ investigation of biofilms}

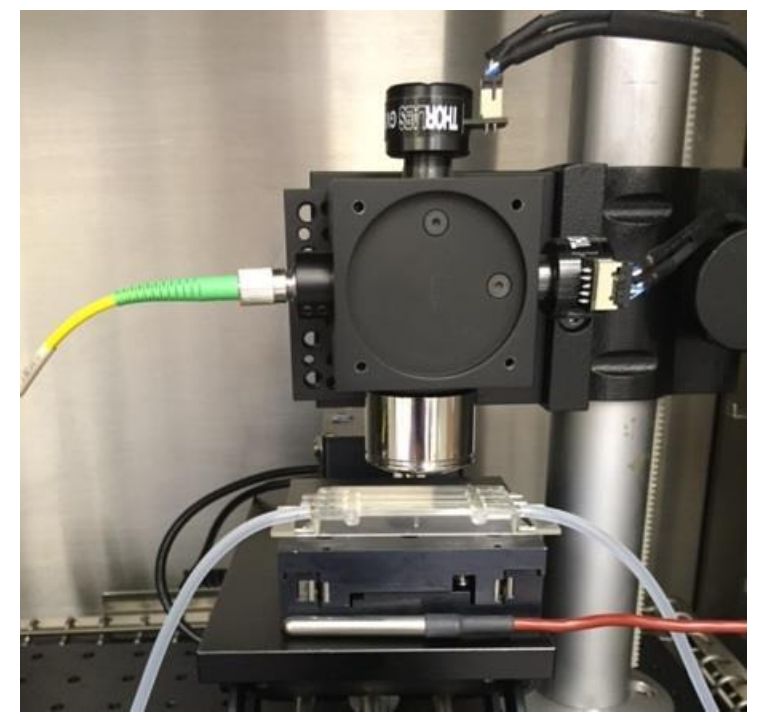

Figure D.1 Selective photograph of the combined experiment, located inside an incubator. The OCM probe is seen located above the flow-cell coverslip, with a working distance of about $1 \mathrm{~cm}$.

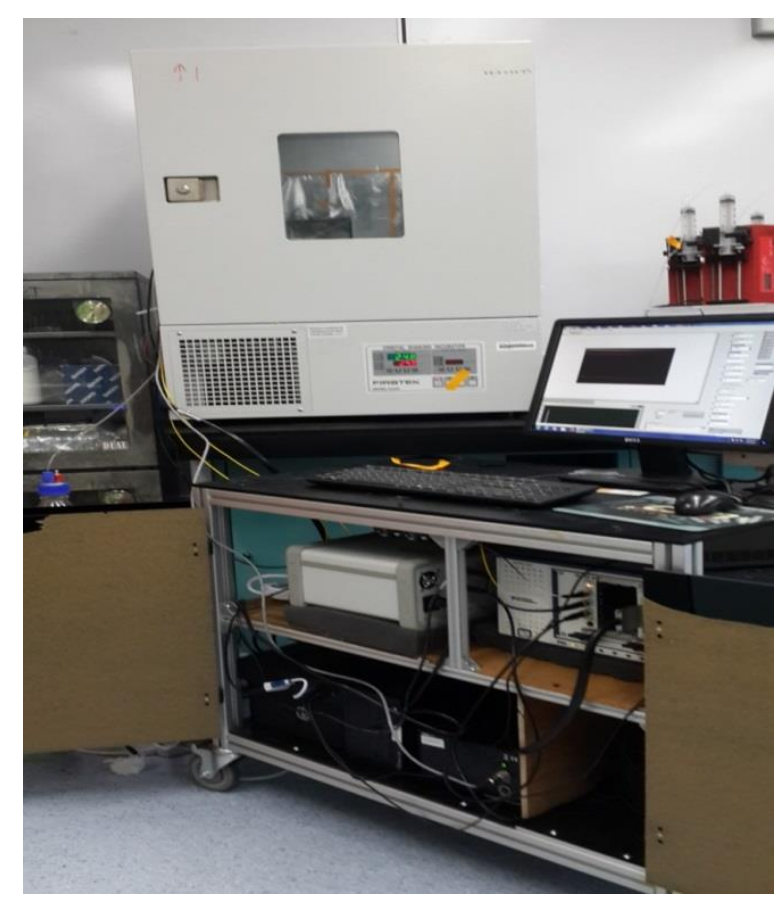

Figure D.2 Photograph of imaging system including data acquisition and control 


\section{Appendix E: Enface images of Klebsiella Pneumonia}

Figure E.1 illustrates the development of the microcolonies of Klebsiella Pneumonia (KP1), at the bottom surface of the flow channel. Figure E.1 (a), (c), (e), (g), (i), (k) and (m) represent the enface images of the bacterial microcolonies. The presence of microcolonies is highlighted by back ground subtraction followed by the edge detection, as shown in Figure E.1. (b), (d), (f), (h), (j), (l) and (n) respectively. Figure E.1 (a), (c) and (e) demonstrates the continuous growth of the KP-1 biofilms in the presence of nutrition medium. On day-3 biofilms have matured and occupied over the entire area of the bottom surface as shown in Figure E.1(e). Figure E.1 (g) represents the image acquired two hours after the perturbation. On day-4 the biomass has reached at its minimum as shown in Figure E.1 (i). The biomass has started recovering on the following days (day-5 and day6), which is evident from Figure E.1 (k) and Figure E.1 (m) respectively.
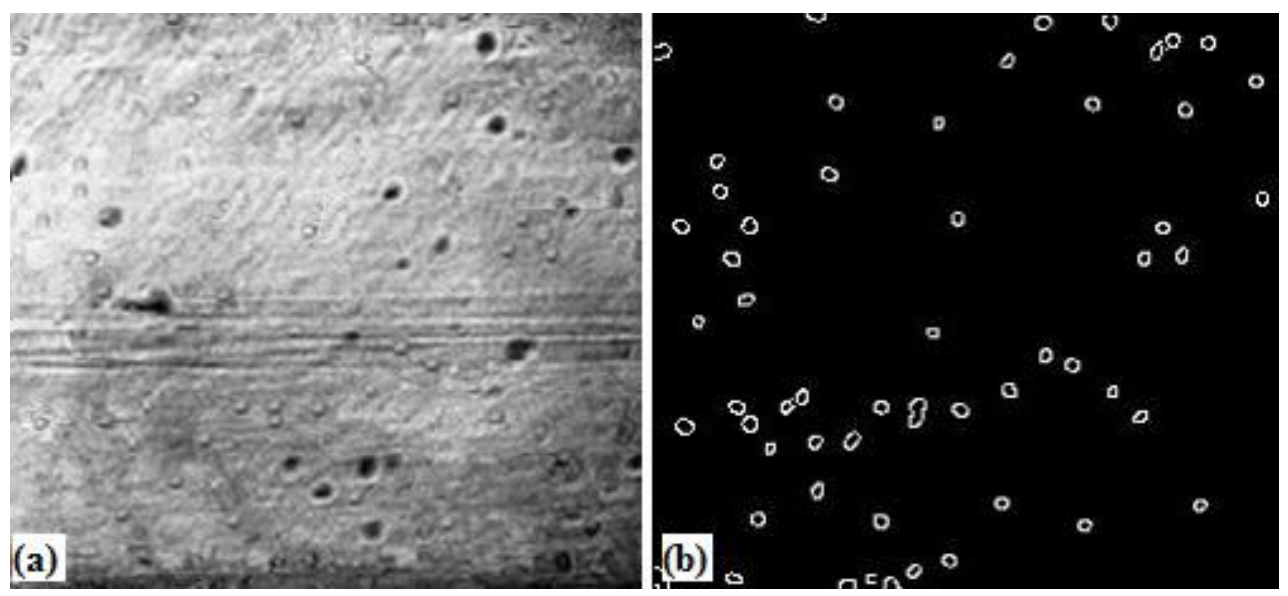

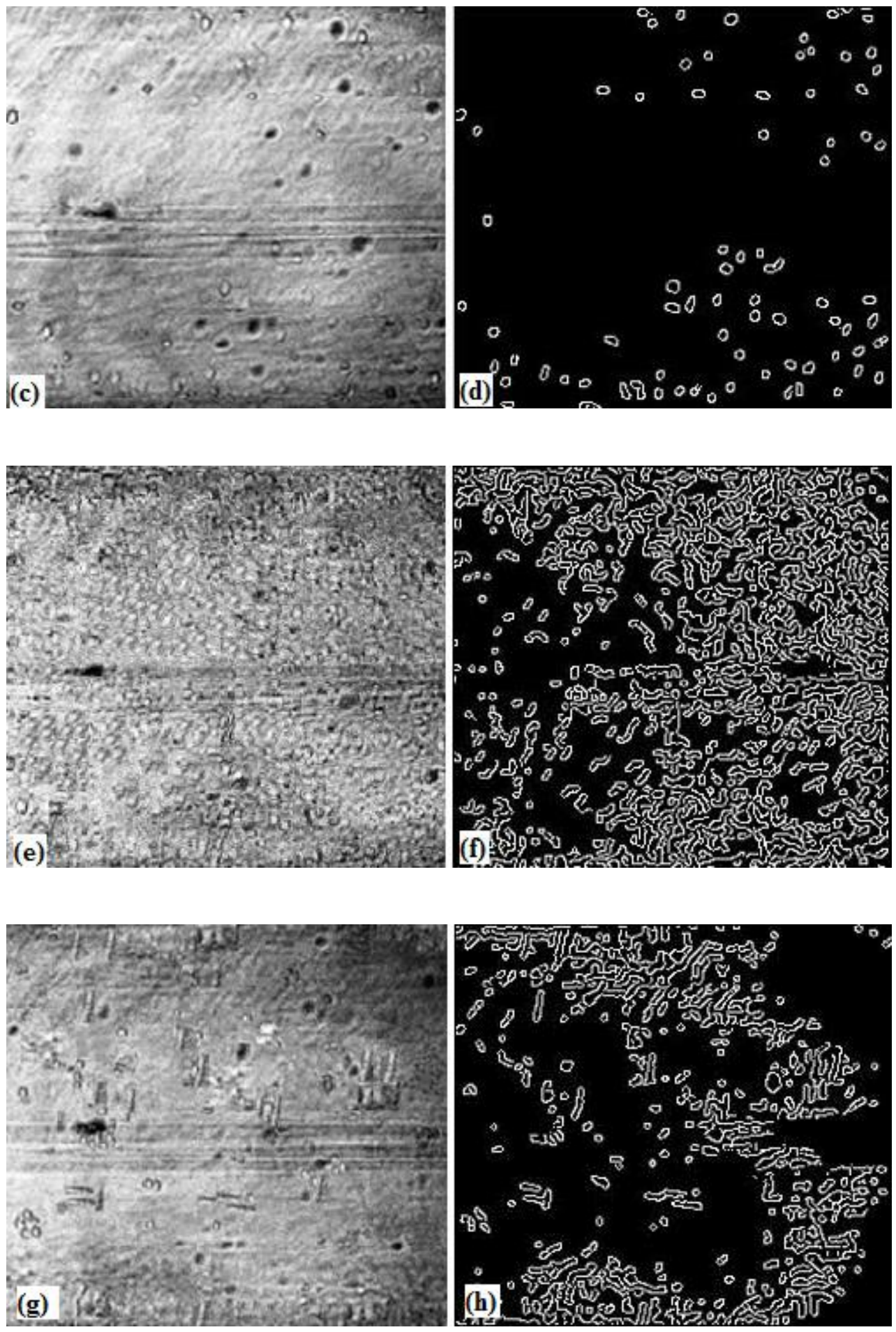

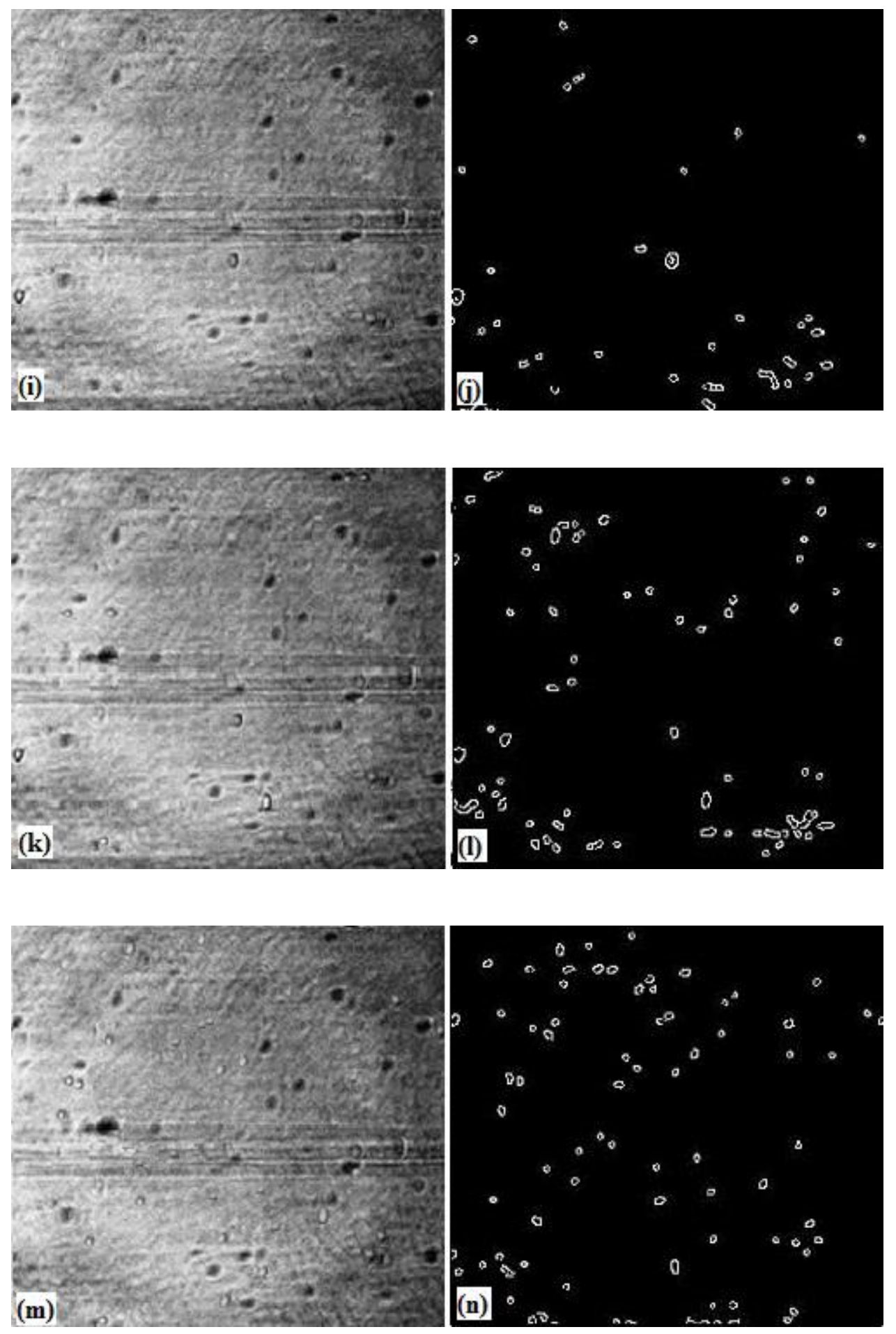

Figure E.1 Enface images of the KP-1 biofilms at the bottom surface of the flow channel 


\section{PUBLICATIONS}

\section{* Peer-Reviewed Journals}

1. Ratheesh Kumar M, Christopher Shearwood, L. K. Seah and Murukeshan V M, "Quantitative optical coherence microscopy for the in situ investigation of the biofilm," Journal of Biomedical Optics 21(12), 127002 (2016). (Published).

2. K. M. Ratheesh, L. K. Seah, and V. M. Murukeshan, "Spectral phase-based automatic calibration scheme for swept source-based optical coherence tomography systems," Physics in Medicine and Biology, 61(21), 7652 (2016). (Published)

3. R. K. Meleppat, Murukeshan. V. Matham, and L. K. Seah, "An efficient phase analysisbased wavenumber linearization scheme for swept source optical coherence tomography systems," Laser Physics Letters 12, 055601 (2015). (Published)

4. K M Ratheesh, P Prabhathan, L K Seah and V M Murukeshan, 'Gold nanorods with higher aspect ratio as potential agent for contrast enhancement and for photothermal applications at $1300 \mathrm{~nm}$ imaging window", Biomedical Physics and Engineering Express 2, 055005 (2016). (Published)

5. Ratheesh Kumar Meleppat, Prabhathan Patinharekandy, Seah Leong Keey, and Murukeshan Vadakke Matham, "Plasmon resonant silica coated silver nanoplates as contrast agents for optical coherence tomography," Journal of Biomedical Nanotechnology 12, 1929-1937 (2016). (Published)

6. Ratheesh Kumar Meleppat, Murukeshan Vadakke Matham, and Seah Leong Keey, "Swept source based optical coherence tomography system at 1320nm-A comprehensive analysis of the opto-mechatronic instrumentation scheme", Asian Journal of Physics, Vol. 24, No 10 (2015)1403-1418 (Published)

7. Ratheesh Kumar M, Murukeshan. V. Matham and L. K. Seah, Non-reflective arm based frequency-domain system for in-vivo imaging applications" Review of Scientific Instruments, (2016). (To be submitted)

8. K M Ratheesh, L K Seah and V M Murukeshan, "Gold nanorod based contrast enhancement in anterior chamber imaging", Journal of Plasmonics (2016). (To be submitted) 


\section{* Conferences Proceedings}

1. Ratheesh Kumar M, Murukeshan. V. Matham, and L. K. Seah, "Optical frequency domain imaging with a rapidly swept laser in the 1300nm bio-imaging window", SPIE Proc. ICOPEN, Singapore (2015).

2. Ratheesh Kumar M, Murukeshan. V. Matham, L. K. Seah and C. Shearwood "Quantification of biofilm thickness using a swept source based optical coherence tomography system ", SPIE Proc. ICOPEN, Singapore (2015).

3. Ratheesh Kumar M, Murukeshan. V. Matham, and L. K. Seah, "Spectral based direct time domain interpolation scheme for resampling in swept source based optical coherence tomography system”, SPIE Proc., Biophotonics, Japan (2015).

4. Ratheesh Kumar M, Murukeshan. V. Matham, and L. K. Seah, "Contrast enhancement in optical coherence tomography at 1300 nm using gold nanorods", SPIE Proc., Biophotonics, Japan (2015). 


\section{REFERENCES}

1. D. Huang, E. A. Swanson, C. P. Lin, J. S. Schuman, W. G. Stinson, W. Chang, M. R. Hee, T. Flotte, K. Gregory, C. A. Puliafito, and et al., "Optical coherence tomography," Science (New York, N.Y.) 254, 1178-1181 (1991).

2. C. Kerbage, H. Lim, W. Sun, M. Mujat, and J. F. de Boer, "Large depth-high resolution full 3D imaging of the anterior segments of the eye using high speed optical frequency domain imaging," Optics Express 15, 7117-7125 (2007).

3. J. G. Fujimoto, C. Pitris, S. A. Boppart, and M. E. Brezinski, "Optical Coherence Tomography: An Emerging Technology for Biomedical Imaging and Optical Biopsy," Neoplasia (New York, N.Y.) 2, 9-25 (2000).

4. M. Hughes; A. Gh. Podoleanu "Simplified dynamic focus method for time domain OCT," Electronics Letters 45, 623 (2009).

5. M. Wojtkowski, R. Leitgeb, A. Kowalczyk, T. Bajraszewski, and A. F. Fercher, "In vivo human retinal imaging by Fourier domain optical coherence tomography," Journal of Biomedical Optics 7, 457-463 (2002).

6. S. Yun, G. Tearney, J. de Boer, N. Iftimia, and B. Bouma, "High-speed optical frequency-domain imaging," Opt. Express 11, 2953-2963 (2003).

7. RK Wang "In vivo full range complex Fourier domain optical coherence tomography," Applied Physics Letters 90, 054103 (2007).

8. V. J. Srinivasan, R. Huber, I. Gorczynska, J. G. Fujimoto, J. Y. Jiang, P. Reisen, and A. E. Cable, "High-speed, high-resolution optical coherence tomography retinal imaging with a frequency-swept laser at $850 \mathrm{~nm}$," Opt. Lett. 32, 361-363 (2007).

9. H. Lim, T. C. Chen, J. F. de Boer, M. Mujat, C. Kerbage, E. C. W. Lee, and Y. Chen, "High-speed imaging of human retina in vivo with swept-source optical coherence tomography," Optics Express 14, 12902-12908 (2006).

10. S. R. Chinn, E. A. Swanson, and J. G. Fujimoto, "Optical coherence tomography using a frequency-tunable optical source," Optics Letters 22, 340-342 (1997).

11. R. Leitgeb, C. Hitzenberger, and A. Fercher, "Performance of fourier domain vs. time domain optical coherence tomography," Opt. Express 11, 889-894 (2003).

12. M. Choma, M. Sarunic, C. Yang, and J. Izatt, "Sensitivity advantage of swept source and Fourier domain optical coherence tomography," Opt. Express 11, 2183-2189 (2003). 
13. V. Jayaraman, G. D. Cole, M. Robertson, C. Burgner, D. John, A. Uddin, and A. Cable, "Rapidly swept, ultra-widely-tunable 1060 nm MEMS-VCSELs," Electronics letters 48, 1331-1333 (2012).

14. V. Jayaraman, D. D. John, C. Burgner, M. E. Robertson, B. Potsaid, J. Y. Jiang, T. H. Tsai, W. Choi, C. D. Lu, P. J. S. Heim, J. G. Fujimoto, and A. E. Cable, "Recent advances in MEMS-VCSELs for high performance structural and functional SS-OCT imaging," Proc. SPIE, 893402-893411, (2014).

15. I Grulkowski, "Retinal, anterior segment and full eye imaging using ultrahigh speed swept source OCT with vertical-cavity surface emitting lasers," Biomedical Optics Express 3, 2733 (2012).

16. C. M. Eigenwillig, B. R. Biedermann, G. Palte, and R. Huber, "K-space linear Fourier domain mode locked laser and applications for optical coherence tomography," Opt. Express 16, 8916-8937 (2008).

17. E. Azimi, B. Liu, and M. E. Brezinski, "Real-time and high-performance calibration method for high-speed swept-source optical coherence tomography," Journal of biomedical optics 15, 016005 (2010).

18. S. H. Yun, C. Boudoux, G. J. Tearney, and B. E. Bouma, "High-speed wavelength-swept semiconductor laser with a polygon-scanner-based wavelength filter," Optics Letters 28, 1981-1983 (2003).

19. C. Dorrer, N. Belabas, J.-P. Likforman, and M. Joffre, "Spectral resolution and sampling issues in Fourier-transform spectral interferometry," J. Opt. Soc. Am. B 17, 1795-1802 (2000).

20. S.-W. Lee, H.-W. Song, B.-K. Kim, M.-Y. Jung, S.-H. Kim, J.-D. Cho, and C.-S. Kim, "Fourier Domain Optical Coherence Tomography for Retinal Imaging with 800-nm Swept Source: Real-time Resampling in k-domain," Journal of the Optical Society of Korea 15, 293-299 (2011).

21. J. Xi, L. Huo, J. Li, and X. Li, "Generic real-time uniform K-space sampling method for high-speed swept-Source optical coherence tomography," Opt. Express 18, 9511-9517 (2010).

22. M. A. Choma, K. Hsu, and J. A. Izatt, "Swept source optical coherence tomography using an all-fiber 1300-nm ring laser source," Journal of biomedical optics 10, 044006044009 (2005).

23. R. Huber, M. Wojtkowski, K. Taira, J. Fujimoto, and K. Hsu, "Amplified, frequency swept lasers for frequency domain reflectometry and OCT imaging: design and scaling principles," Opt Express 13, 3513-3528 (2005). 
24. R. Huber, M. Wojtkowski, J. G. Fujimoto, J. Y. Jiang, and A. E. Cable, "Threedimensional and C-mode OCT imaging with a compact, frequency swept laser source at 1300 nm," Opt. Express 13, 10523-10538 (2005).

25. T. Wu, Z. Ding, L. Wang, and M. Chen, "Spectral phase based k-domain interpolation for uniform sampling in swept-source optical coherence tomography," Opt. Express 19, 18430-18439 (2011).

26. T. Wu, Z. Ding, K. Wang, and C. Wang, "Swept source optical coherence tomography based on non-uniform discrete fourier transform," Chin. Opt. Lett. 7, 941-944 (2009).

27. K. Wang, Z. Ding, T. Wu, C. Wang, J. Meng, M. Chen, and L. Xu, "Development of a non-uniform discrete Fourier transform based high speed spectral domain optical coherence tomography system," Opt. Express 17, 12121-12131 (2009).

28. M. Maciejewski, M. Strąkowski, J. Pluciński, and B. B. Kosmowski, "Dispersion compensation in optical coherence tomography," Proc. SPIE, 63471-63474, (2006).

29. K.-S. Lee, A. C. Akcay, T. Delemos, E. Clarkson, and J. P. Rolland, "Dispersion control with a Fourier-domain optical delay line in a fiber-optic imaging interferometer," Applied Optics 44, 4009-4022 (2005).

30. N. Lippok, S. Coen, P. Nielsen, and F. Vanholsbeeck, "Dispersion compensation in Fourier domain optical coherence tomography using the fractional Fourier transform," Optics Express 20, 23398-23413 (2012).

31. A. F. Fercher, C. K. Hitzenberger, M. Sticker, R. Zawadzki, B. Karamata, and T. Lasser, "Dispersion compensation for optical coherence tomography depth-scan signals by a numerical technique," Optics Communications 204, 67-74 (2002).

32. M. Wojtkowski, V. J. Srinivasan, T. H. Ko, J. G. Fujimoto, A. Kowalczyk, and J. S. Duker, "Ultrahigh-resolution, high-speed, Fourier domain optical coherence tomography and methods for dispersion compensation," Optics Express 12, 2404-2422 (2004).

33. T. Sandu, "Shape effects on localized surface plasmon resonances in metallic nanoparticles," J Nanopart Res 14, 1-10 (2012).

34. E. Ringe, J. M. McMahon, K. Sohn, C. Cobley, Y. Xia, J. Huang, G. C. Schatz, L. D. Marks, and R. P. Van Duyne, "Unraveling the Effects of Size, Composition, and Substrate on the Localized Surface Plasmon Resonance Frequencies of Gold and Silver Nanocubes: A Systematic Single-Particle Approach," The Journal of Physical Chemistry C 114, 12511-12516 (2010).

35. M.-C. Daniel, and D. Astruc, "Gold Nanoparticles: Assembly, Supramolecular Chemistry, Quantum-Size-Related Properties, and Applications toward Biology, Catalysis, and Nanotechnology," Chemical Reviews 104, 293-346 (2004). 
36. X. Huang, and M. A. El-Sayed, "Gold nanoparticles: Optical properties and implementations in cancer diagnosis and photothermal therapy," Journal of Advanced Research 1, 13-28 (2010).

37. C. A. Banzhaf, L. Themstrup, H. C. Ring, M. Mogensen, and G. B. E. Jemec, "Optical coherence tomography imaging of non-melanoma skin cancer undergoing imiquimod therapy," Skin Research and Technology 20, 170-176 (2014).

38. O. Assayag, K. Grieve, B. Devaux, F. Harms, J. Pallud, F. Chretien, C. Boccara, and P. Varlet, "Imaging of non-tumorous and tumorous human brain tissues with full-field optical coherence tomography," NeuroImage: Clinical 2, 549-557 (2013).

39. A. Oldenburg, D. A. Zweifel, C. Xu, A. Wei, and S. A. Boppart, "Characterization of plasmon-resonant gold nanorods as near-infrared optical contrast agents investigated using a double-integrating sphere system," Proc. SPIE, 50-60, (2005).

40. J. W. Pickering, S. A. Prahl, N. van Wieringen, J. F. Beek, H. J. C. M. Sterenborg, and M. J. C. van Gemert, "Double-integrating-sphere system for measuring the optical properties of tissue," Applied Optics 32, 399-410 (1993).

41. E. Tomaszewska, K. Soliwoda, K. Kadziola, B. Tkacz-Szczesna, G. Celichowski, M. Cichomski, W. Szmaja, and J. Grobelny, "Detection Limits of DLS and UV-Vis Spectroscopy in Characterization of Polydisperse Nanoparticles Colloids," Journal of Nanomaterials 2013, 10 (2013).

42. K. J. Lee, P. D. Nallathamby, L. M. Browning, C. J. Osgood, and X.-H. N. Xu, "In Vivo Imaging of Transport and Biocompatibility of Single Silver Nanoparticles in Early Development of Zebrafish Embryos," ACS Nano 1, 133-143 (2007).

43. X.-H. N. Xu, W. J. Brownlow, S. V. Kyriacou, Q. Wan, and J. J. Viola, "Real-Time Probing of Membrane Transport in Living Microbial Cells Using Single Nanoparticle Optics and Living Cell Imaging," Biochemistry 43, 10400-10413 (2004).

44. F. V. B. Manz, D. Goll and H. Horn, "Investigation of biofilm structure, flow patterns and detachment with magnetic resonance imaging," Water Science \& Technology 52, 1$6(2005)$.

45. T. Schmid, U. Panne, C. Haisch, M. Hausner, and R. Niessner, "A Photoacoustic Technique for Depth-Resolved In Situ Monitoring of Biofilms," Environmental Science \& Technology 36, 4135-4141 (2002).

46. J. R. Lawrence, G. D. W. Swerhone, G. G. Leppard, T. Araki, X. Zhang, M. M. West, and A. P. Hitchcock, "Scanning Transmission X-Ray, Laser Scanning, and Transmission Electron Microscopy Mapping of the Exopolymeric Matrix of Microbial Biofilms," Applied and Environmental Microbiology 69, 5543-5554 (2003). 
47. R. Bakke, R. Kommedal, and S. Kalvenes, "Quantification of biofilm accumulation by an optical approach," Journal of Microbiological Methods 44, 13-26 (2001).

48. C. Haisch, and R. Niessner, "Visualisation of transient processes in biofilms by optical coherence tomography," Water Research 41, 2467-2472 (2007).

49. F. Blauert, H. Horn, and M. Wagner, "Time-resolved biofilm deformation measurements using optical coherence tomography," Biotechnology and Bioengineering 112, 18931905 (2015).

50. H. L. K. J. Beutel, and R. L. Van Metter, Handbook of Medical Imaging ( SPIE Press, 2000).

51. M. Gerd, and S. K. Joel, "Positron emission tomography," Physics in Medicine and Biology 51, R117 (2006).

52. T. Wilson, Confocal microscopy (Academic Press, 1990).

53. D. L. H. W. R. Hedrick, and D. E. Starchmann, Ultrasound Physics and Intrumentation 4th edition (Mosby, 2005).

54. W. D. a. J. G. Fujimoto, Optical Coherence Tomography: Technology and Applications (Springer, 2008).

55. W. Drexler, U. Morgner, R. K. Ghanta, F. X. Kärtner, J. S. Schuman, and J. G. Fujimoto, "Ultrahigh-resolution ophthalmic optical coherence tomography," Nature medicine 7, 502-507 (2001).

56. A. P. Bruckner, "Picosecond light scattering measurements of cataract microstructure," Applied Optics 17, 3177-3183 (1978).

57. J. G. Fujimoto, C. A. Puliafito, R. Margolis, A. Oseroff, S. De Silvestri, and E. P. Ippen, "Femtosecond optical ranging in biological systems," Optics Letters 11, 150-152 (1986).

58. R. C. Youngquist, S. Carr, and D. E. N. Davies, "Optical coherence-domain reflectometry: a new optical evaluation technique," Optics Letters 12, 158-160 (1987).

59. K. Takada, I. Yokohama, K. Chida, and J. Noda, "New measurement system for fault location in optical waveguide devices based on an interferometric technique," Applied Optics 26, 1603-1606 (1987).

60. A. F. Fercher, K. Mengedoht, and W. Werner, "Eye-length measurement by interferometry with partially coherent light," Optics Letters 13, 186-188 (1988).

61. L. J. Steven, "Optical properties of biological tissues: a review," Physics in Medicine and Biology 58, R37 (2013). 
62. B. Považay, K. Bizheva, B. Hermann, A. Unterhuber, H. Sattmann, A. F. Fercher, W. Drexler, C. Schubert, P. K. Ahnelt, M. Mei, R. Holzwarth, W. J. Wadsworth, J. C. Knight, and P. S. J. Russel, "Enhanced visualization of choroidal vessels using ultrahigh resolution ophthalmic OCT at 1050 nm," Optics Express 11, 1980-1986 (2003).

63. G. M. Hale, and M. R. Querry, "Optical Constants of Water in the 200-nm to 200- $\mu \mathrm{m}$ Wavelength Region," Applied Optics 12, 555-563 (1973).

64. M. E. Brezinski, G. J. Tearney, B. E. Bouma, J. A. Izatt, M. R. Hee, E. A. Swanson, J. F. Southern, and J. G. Fujimoto, "Optical Coherence Tomography for Optical Biopsy," Circulation 93, 1206-1213 (1996).

65. J. G. Fujimoto, M. E. Brezinski, G. J. Tearney, S. A. Boppart, B. Bouma, M. R. Hee, J. F. Southern, and E. A. Swanson, "Optical biopsy and imaging using optical coherence tomography," Nat Med 1, 970-972 (1995).

66. J. M. Schmitt, A. Knuttel, M. Yadlowsky, and M. A. Eckhaus, "Optical-coherence tomography of a dense tissue: statistics of attenuation and backscattering," Physics in Medicine and Biology 39, 1705 (1994).

67. A. F. Fercher, C. K. Hitzenberger, G. Kamp, and S. Y. El-Zaiat, "Measurement of intraocular distances by backscattering spectral interferometry," Optics Communications 117, 43-48 (1995).

68. B. Golubovic, B. E. Bouma, G. J. Tearney, and J. G. Fujimoto, "Optical frequencydomain reflectometry using rapid wavelength tuning of a Cr4+:forsterite laser," Optics Letters 22, 1704-1706 (1997).

69. A. M. Rollins, and J. A. Izatt, "Optimal interferometer designs for optical coherence tomography," Optics Letters 24, 1484-1486 (1999).

70. U. Sharma, J. U. Kang, and N. M. Fried, "Fizeau Optical Coherence Tomography: Sensitivity Optimization and System Analysis," Proc. OSA, 11-15, (2005).

71. B. E. Bouma, and G. J. Tearney, "Power-efficient nonreciprocal interferometer and linear-scanning fiber-optic catheter for optical coherence tomography," Optics Letters 24, 531-533 (1999).

72. A. G. Podoleanu, "Unbalanced versus balanced operation in an optical coherence tomography system," Applied Optics 39, 173-182 (2000).

73. S.-W. Huang, A. D. Aguirre, R. A. Huber, D. C. Adler, and J. G. Fujimoto, "Swept source optical coherence microscopy using a Fourier domain mode-locked laser," Optics Express 15, 6210-6217 (2007).

74. J.-U. Kang, J.-H. Han, X. Liu, and K. Zhang, "Common-path Optical Coherence Tomography for Biomedical Imaging and Sensing," Journal of the Optical Society of Korea 14, 1-13 (2010). 
75. A. B. Vakhtin, D. J. Kane, W. R. Wood, and K. A. Peterson, "Common-path interferometer for frequency-domain optical coherence tomography," Applied Optics 42, 6953-6958 (2003).

76. J. U. Kang, J.-H. Han, X. Liu, K. Zhang, C. G. Song, and P. Gehlbach, "Endoscopic Functional Fourier Domain Common Path Optical Coherence Tomography for Microsurgery," IEEE journal of selected topics in quantum electronics : a publication of the IEEE Lasers and Electro-optics Society 16, 781-792 (2010).

77. J. H. Han, X. Liu, Z. Kang, and J. U. Kang, "Common-path fourier-domain optical coherence tomography in ophthalmology applications," Proc. IEEE, 163-166 (2009).

78. Y. Huang, X. Liu, C. Song, and J. U. Kang, "Motion-compensated hand-held commonpath Fourier-domain optical coherence tomography probe for image-guided intervention," Biomedical Optics Express 3, 3105-3118 (2012).

79. X. Liu, X. Li, D.-H. Kim, I. Ilev, and J. U. Kang, "Fiber-optic Fourier-domain commonpath OCT," Chinese Optics Letters 6, 899-901 (2008).

80. K. M. Tan, M. Mazilu, T. H. Chow, W. M. Lee, K. Taguchi, B. K. Ng, W. Sibbett, C. S. Herrington, C. T. A. Brown, and K. Dholakia, "In-fiber common-path optical coherence tomography using a conical-tip fiber," Optics Express 17, 2375-2384 (2009).

81. D. C. Adler, Y. Chen, R. Huber, J. Schmitt, J. Connolly, and J. G. Fujimoto, "Threedimensional endomicroscopy using optical coherence tomography," Nat Photon 1, 709716 (2007).

82. T. Klein, W. Wieser, C. M. Eigenwillig, B. R. Biedermann, and R. Huber, "Megahertz OCT for ultrawide-field retinal imaging with a 1050nm Fourier domain mode-locked laser," Optics Express 19, 3044-3062 (2011).

83. F. Lexer, C. K. Hitzenberger, W. Drexler, S. Molebny, H. Sattmann, M. Sticker, and A. F. Fercher, "Dynamic coherent focus OCT with depth-independent transversal resolution," Journal of Modern Optics 46, 541-553 (1999).

84. S. H. Yun, G. Tearney, J. de Boer, and B. Bouma, "Motion artifacts in optical coherence tomography with frequency-domain ranging," Optics Express 12, 2977-2998 (2004).

85. H. C. Hendargo, R. P. McNabb, A.-H. Dhalla, N. Shepherd, and J. A. Izatt, "Doppler velocity detection limitations in spectrometer-based versus swept-source optical coherence tomography," Biomedical Optics Express 2, 2175-2188 (2011).

86. P. C. Schuman, Fujimoto J.G., Optical Coherence Tomography of Ocular Diseases, 2nd ed (Slack Inc, 2004). 
87. I. Grulkowski, M. Gora, M. Szkulmowski, I. Gorczynska, D. Szlag, S. Marcos, A. Kowalczyk, and M. Wojtkowski, "Anterior segment imaging with Spectral OCT system using a high-speed CMOS camera," Optics Express 17, 4842-4858 (2009).

88. J. A. Goldsmith, Y. Li, M. R. Chalita, V. Westphal, C. A. Patil, A. M. Rollins, J. A. Izatt, and D. Huang, "Anterior Chamber Width Measurement by High-Speed Optical Coherence Tomography," Ophthalmology 112, 238-244 (2005).

89. H. Hoerauf, C. Wirbelauer, C. Scholz, R. Engelhardt, P. Koch, H. Laqua, and R. Birngruber, "Slit-lamp-adapted optical coherence tomography of the anterior segment," Graefe's Archive for Clinical and Experimental Ophthalmology 238, 8-18 (2000).

90. S. Radhakrishnan, A. M. Rollins, J. E. Roth, and et al., "REal-time optical coherence tomography of the anterior segment at $1310 \mathrm{~nm}, "$ Archives of Ophthalmology 119, 1179-1185 (2001).

91. B. E. Bouma, G. J. Tearney, H. Yabushita, M. Shishkov, C. R. Kauffman, D. DeJoseph Gauthier, B. D. MacNeill, S. L. Houser, H. T. Aretz, E. F. Halpern, and I.-K. Jang, "Evaluation of intracoronary stenting by intravascular optical coherence tomography," Heart 89, 317-320 (2003).

92. T. Wang, T. Pfeiffer, E. Regar, W. Wieser, H. van Beusekom, C. T. Lancee, G. Springeling, I. Krabbendam, A. F. W. van der Steen, R. Huber, and G. van Soest, "Heartbeat OCT: in vivo intravascular megahertz-optical coherence tomography," Biomedical Optics Express 6, 5021-5032 (2015).

93. M. Asrar ul Haq, J. Layland, V. Mutha, and P. Barlis, "The invasive assessment of coronary atherosclerosis and stents using optical coherence tomography: a clinical update," Heart Asia 5, 154-161 (2013).

94. A. M. Rollins, R. Ung-arunyawee, A. Chak, R. C. K. Wong, K. Kobayashi, M. V. Sivak, and J. A. Izatt, "Real-time in vivo imaging of human gastrointestinal ultrastructure by use of endoscopic optical coherence tomography with a novel efficient interferometer design," Optics Letters 24, 1358-1360 (1999).

95. A. V. Shakhov, A. B. Terentjeva, V. A. Kamensky, L. B. Snopova, V. M. Gelikonov, F. I. Feldchtein, and A. M. Sergeev, "Optical coherence tomography monitoring for laser surgery of laryngeal carcinoma," Journal of Surgical Oncology 77, 253-258 (2001).

96. A. G. Bibas, A. G. Podoleanu, R. G. Cucu, M. Bonmarin, G. M. Dobre, V. M. M. Ward, E. Odell, A. Boxer, M. J. Gleeson, and D. A. Jackson, "3-D optical coherence tomography of the laryngeal mucosa*," Clinical Otolaryngology \& Allied Sciences 29, 713-720 (2004).

97. N. Hanna, D. Saltzman, D. Mukai, Z. Chen, S. Sasse, J. Milliken, S. Guo, W. Jung, H. Colt, and M. Brenner, "Two-dimensional and 3-dimensional optical coherence 
tomographic imaging of the airway, lung, and pleura," The Journal of Thoracic and Cardiovascular Surgery 129, 615-622, (2009).

98. G. J. Tearney, M. E. Brezinski, J. F. Southern, B. E. Bouma, S. A. Boppart, and J. G. Fujimoto, "Optical Biopsy in Human Urologic Tissue Using Optical Coherence Tomography," The Journal of Urology 157, 1915-1919, (2009).

99. C. Pitris, M. E. Brezinski, B. E. Bouma, G. J. Tearney, J. F. Southern, and J. G. Fujimoto, "High Resolution Imaging of the Upper Respiratory Tract with Optical Coherence Tomography," American Journal of Respiratory and Critical Care Medicine 157, 1640-1644 (1998).

100. A. M. Sergeev, V. M. Gelikonov, G. V. Gelikonov, F. I. Feldchtein, R. V. Kuranov, N. D. Gladkova, N. M. Shakhova, L. B. Snopova, A. V. Shakhov, I. A. Kuznetzova, A. N. Denisenko, V. V. Pochinko, Y. P. Chumakov, and O. S. Streltzova, "In vivo endoscopic OCT imaging of precancer and cancer states of human mucosa," Optics Express 1, 432440 (1997).

101. F.-Y. Chang, M.-T. Tsai, Z.-Y. Wang, C.-K. Chi, C.-K. Lee, C.-H. Yang, M.-C. Chan, and Y.-J. Lee, "Optical coherence tomography-guided laser microsurgery for blood coagulation with continuous-wave laser diode," Scientific Reports 5, 16739 (2015).

102. M. W. Jenkins, O. Q. Chughtai, A. N. Basavanhally, M. Watanabe, and A. M. Rollins, "In vivo gated 4D imaging of the embryonic heart using optical coherence tomography," Journal of Biomedical Optics 12, $030505-030503$ (2007).

103. S. A. Boppart, M. E. Brezinski, B. E. Bouma, G. J. Tearney, and J. G. Fujimoto, "Investigation of Developing Embryonic Morphology Using Optical Coherence Tomography," Developmental Biology 177, 54-63 (1996).

104. E. A. Swanson, D. Huang, C. P. Lin, C. A. Puliafito, M. R. Hee, and J. G. Fujimoto, "High-speed optical coherence domain reflectometry," Optics Letters 17, 151-153 (1992).

105. M. W. D. Douglas B. Murphy, Fundamentals of Light Microscopy and Electronic Imaging, 2nd Edition (Wiley-Blackwell, 2011).

106. X. Liu, M. Balicki, R. H. Taylor, and J. U. Kang, "Towards automatic calibration of Fourier-Domain OCT for robot-assisted vitreoretinal surgery," Opt. Express 18, 2433124343 (2010).

107. J. Zhang, J. S. Nelson, and Z. Chen, "Removal of a mirror image and enhancement of the signal-to-noise ratioin Fourier-domain optical coherence tomography using an electro-optic phasemodulator," Opt. Lett. 30, 147-149 (2005).

108. Y. Zhang, X. Li, L. Wei, K. Wang, Z. Ding, and G. Shi, "Time-domain interpolation for Fourier-domain optical coherence tomography," Optics Letters 34, 1849-1851 (2009). 
109. S. Vergnole, D. Lévesque, and G. Lamouche, "Experimental validation of an optimized signal processing method to handle non-linearity in swept-source optical coherence tomography," Opt. Express 18, 10446-10461 (2010).

110. M. Gora, K. Karnowski, M. Szkulmowski, B. J. Kaluzny, R. Huber, A. Kowalczyk, and M. Wojtkowski, "Ultra high-speed swept source OCT imaging of the anterior segment of human eye at $200 \mathrm{kHz}$ with adjustable imaging range," Opt Express 17, 14880-14894 (2009).

111. V. M. Gelikonov, G. V. Gelikonov, and P. A. Shilyagin, "Linear-wavenumber spectrometer for high-speed spectral-domain optical coherence tomography," Opt. Spectrosc. 106, 459-465 (2009).

112. N. Zhang, T. Chen, C. Wang, J. Zhang, T. Huo, J. Zheng, and P. Xue, "Spectral-domain optical coherence tomography with a Fresnel spectrometer," Opt. Lett. 37, 1307-1309 (2012).

113. T. Huo, J. Zhang, J.-g. Zheng, T. Chen, C. Wang, N. Zhang, W. Liao, X. Zhang, and P. Xue, "Linear-in-wavenumber swept laser with an acousto-optic deflector for optical coherence tomography," Opt. Lett. 39, 247-250 (2014).

114. K. Zhang, and J. U. Kang, "Graphics processing unit accelerated non-uniform fast Fourier transform for ultrahigh-speed, real-time Fourier-domain OCT," Opt. Express 18, 23472-23487 (2010).

115. J. K. Barton, K. W. Gossage, W. Xu, J. R. Ranger-Moore, K. Saboda, C. A. Brooks, L. D. Duckett, S. J. Salasche, J. A. Warneke, and D. S. Alberts, "Investigating sundamaged skin and actinic keratosis with optical coherence tomography: a pilot study," Technology in cancer research \& treatment 2, 525-535 (2003).

116. A. R. Tumlinson, L. P. Hariri, U. Utzinger, and J. K. Barton, "Miniature endoscope for simultaneous optical coherence tomography and laser-induced fluorescence measurement," Applied Optics 43, 113-121 (2004).

117. J. A. Linehan, E. R. Bracamonte, L. P. Hariri, M. H. Sokoloff, P. S. Rice, J. K. Barton, and M. M. Nguyen, "Feasibility of optical coherence tomography imaging to characterize renal neoplasms: limitations in resolution and depth of penetration," BJU international 108, 1820-1824 (2011).

118. A. K. Singh, B. Chakravarty, and K. Chaudhury, "Nanoparticle-Assisted Combinatorial Therapy for Effective Treatment of Endometriosis," Journal of Biomedical Nanotechnology 11, 789-804 (2015).

119. L. Ferroni, C. Gardin, A. D. Puppa, S. Sivolella, G. Brunello, R. Scienza, E. Bressan, D. D'Avella, and B. Zavan, "Novel Nanotechnologies for Brain Cancer Therapeutics and Imaging," Journal of Biomedical Nanotechnology 11, 1899-1912 (2015). 
120. V. V. Tuchin, G. B. Altshuler, A. A. Gavrilova, A. B. Pravdin, D. Tabatadze, J. Childs, and I. V. Yaroslavsky, "Optical clearing of skin using flashlamp-induced enhancement of epidermal permeability," Lasers in Surgery and Medicine 38, 824-836 (2006).

121. I. V. Larina, E. F. Carbajal, V. V. Tuchin, M. E. Dickinson, and K. V. Larin, "Enhanced OCT imaging of embryonic tissue with optical clearing," Laser Physics Letters 5, 476 (2008).

122. J. P. Ehlers, P. K. Gupta, S. Farsiu, R. Maldonado, T. Kim, C. A. Toth, and P. Mruthyunjaya, "Evaluation of Contrast Agents for Enhanced Visualization in Optical Coherence Tomography," Investigative Ophthalmology \& Visual Science 51, 6614-6619 (2010).

123. T. M. Lee, A. L. Oldenburg, S. Sitafalwalla, D. L. Marks, W. Luo, F. J.-J. Toublan, K. S. Suslick, and S. A. Boppart, "Engineered microsphere contrast agents for optical coherence tomography," Optics Letters 28, 1546-1548 (2003).

124. C. Yang, M. A. Choma, L. E. Lamb, J. D. Simon, and J. A. Izatt, "Protein-based molecular contrast optical coherence tomography with phytochrome as the contrast agent," Optics Letters 29, 1396-1398 (2004).

125. C. Xu, J. Ye, D. L. Marks, and S. A. Boppart, "Near-infrared dyes as contrast-enhancing agents for spectroscopic optical coherence tomography," Optics Letters 29, 1647-1649 (2004).

126. Z. Fan, X. Huang, C. Tan, and H. Zhang, "Thin metal nanostructures: synthesis, properties and applications," Chem Sci 6, 95 - 111 (2015).

127. T. S. Troutman, J. K. Barton, and M. Romanowski, "Optical coherence tomography with plasmon resonant nanorods of gold," Optics Letters 32, 1438-1440 (2007).

128. A. Agrawal, S. Huang, A. Wei Haw Lin, M.-H. Lee, J. K. Barton, R. A. Drezek, and T. J. Pfefer, "Quantitative evaluation of optical coherence tomography signal enhancement with gold nanoshells," Journal of Biomedical Optics 11, 041121-041121-041128 (2006).

129. J. Chen, F. Saeki, B. J. Wiley, H. Cang, M. J. Cobb, Z.-Y. Li, L. Au, H. Zhang, M. B. Kimmey, Li, and Y. Xia, "Gold Nanocages: Bioconjugation and Their Potential Use as Optical Imaging Contrast Agents," Nano Letters 5, 473-477 (2005).

130. E. V. Zagaynova, M. V. Shirmanova, M. Y. Kirillin, B. N. Khlebtsov, A. G. Orlova, I. V. Balalaeva, M. A. Sirotkina, M. L. Bugrova, P. D. Agrba, and V. A. Kamensky, "Contrasting properties of gold nanoparticles for optical coherence tomography: phantom, in vivo studies and Monte Carlo simulation," Physics in Medicine and Biology 53, 4995 (2008).

131. J. Xi, Y. Chen, and X. Li, "Characterizing optical properties of nano contrast agents by using cross-referencing OCT imaging," Biomedical Optics Express 4, 842-851 (2013). 
132. M. D. Rodney, "Biofilms: Microbial Life on Surfaces," Emerging Infectious Disease journal 8, 881 (2002).

133. S. S. Branda, A. Vik, L. Friedman, and R. Kolter, "Biofilms: the matrix revisited," Trends in Microbiology 13, 20-26 (2005).

134. P. S. Stewart, and J. William Costerton, "Antibiotic resistance of bacteria in biofilms," The Lancet 358, 135-138 (2001).

135. B. Manz, F. Volke, D. Goll, and H. Horn, "Measuring local flow velocities and biofilm structure in biofilm systems with Magnetic Resonance Imaging (MRI)," Biotechnology and Bioengineering 84, 424-432 (2003).

136. R. Thar, M. Kühl, and G. Holst, "Fiber-Optic Fluorometer for Microscale Mapping of Photosynthetic Pigments in Microbial Communities," Applied and Environmental Microbiology 67, 2823-2828 (2001).

137. H. Beyenal, C. Yakymyshyn, J. Hyungnak, C. C. Davis, and Z. Lewandowski, "An optical microsensor to measure fluorescent light intensity in biofilms," Journal of Microbiological Methods 58, 367-374 (2004).

138. T. Schmid, C. Helmbrecht, U. Panne, C. Haisch, and R. Niessner, "Process analysis of biofilms by photoacoustic spectroscopy," Analytical and Bioanalytical Chemistry 375, 1124-1129 (2003).

139. D. E. Nivens, J. Q. Chambers, T. R. Anderson, A. Tunlid, J. Smit, and D. C. White, "Monitoring microbiol adhesion and biofilm formation by attenuated total reflection/Fourier transform infrared spectroscopy," Journal of Microbiological Methods 17, 199-213 (1993).

140. R. Bakke, and P. Q. Olsson, "Biofilm thickness measurements by light microscopy," Journal of Microbiological Methods 5, 93-98 (1986).

141. J. R. Lawrence, D. R. Korber, B. D. Hoyle, J. W. Costerton, and D. E. Caldwell, "Optical sectioning of microbial biofilms," Journal of Bacteriology 173, 6558-6567 (1991).

142. L. Tóth, A. Vajas, P. Csomor, A. Berta, I. Sziklai, and T. Karosi, "Optical coherence tomography for biofilm detection in chronic rhinosinusitis with nasal polyposis," European Archives of Oto-Rhino-Laryngology 270, 555-563 (2012).

143. C. Xi, D. Marks, S. Schlachter, W. Luo, and S. A. Boppart, "High-resolution threedimensional imaging of biofilm development using optical coherence tomography," Journal of Biomedical Optics 11, 034001-034006 (2006). 
144. K. Aljasem, A. Werber, A. Seifert, and H. Zappe, "Fiber optic tunable probe for endoscopic optical coherence tomography," Journal of Optics A: Pure and Applied Optics 10, 044012 (2008).

145. J. W. Costerton, P. S. Stewart, and E. P. Greenberg, "Bacterial Biofilms: A Common Cause of Persistent Infections," Science 284, 1318-1322 (1999).

146. J. M. Schmitt, "Optical coherence tomography (OCT): a review," IEEE Journal of Selected Topics in Quantum Electronics 5, 1205-1215 (1999).

147. G. Hausler, and M. W. Lindner, "“Coherence Radar" and "Spectral Radar"-New Tools for Dermatological Diagnosis," Journal of Biomedical Optics 3, 21-31 (1998).

148. R. J. Zawadzki, S. M. Jones, S. Pilli, S. Balderas-Mata, D. Y. Kim, S. S. Olivier, and J. S. Werner, "Integrated adaptive optics optical coherence tomography and adaptive optics scanning laser ophthalmoscope system for simultaneous cellular resolution in vivo retinal imaging," Biomedical Optics Express 2, 1674-1686 (2011).

149. S. W. Smith, Digital Signal Processing (California Technical Publishing 1999).

150. G. R. Fowles, Introduction to modern optics (Dover Publications, 1989).

151. J. Millstone, S. Hurst, G. Metraux, J. Cutler, and C. Mirkin, "Colloidal gold and silver triangular nanoprisms," Small 5, 646 - 664 (2009).

152. P. K. Jain, X. Huang, I. H. El-Sayed, and M. A. El-Sayed, "Noble Metals on the Nanoscale: Optical and Photothermal Properties and Some Applications in Imaging, Sensing, Biology, and Medicine," Accounts of Chemical Research 41, 1578-1586 (2008).

153. J. Chen, D. Wang, J. Xi, L. Au, A. Siekkinen, A. Warsen, Z.-Y. Li, H. Zhang, Y. Xia, and $\mathrm{X}$. Li, "Immuno Gold Nanocages with Tailored Optical Properties for Targeted Photothermal Destruction of Cancer Cells," Nano Letters 7, 1318-1322 (2007).

154. S. L. Smitha, K. G. Gopchandran, T. R. Ravindran, and V. S. Prasad, "Gold nanorods with finely tunable longitudinal surface plasmon resonance as SERS substrates," Nanotechnology 22, 265705 (2011).

155. H. Chen, L. Shao, Q. Li, and J. Wang, "Gold nanorods and their plasmonic properties," Chemical Society Reviews 42, 2679-2724 (2013).

156. S. Moon, Y. Oh, D. Kim, H. Lee, H. C. Kim, and K. Lee, "Gold @ silica core-shell nanoparticle for enhanced surface plasmon resonance detection of DNA hybridization in combination with gold nanowire gratings",Proc. SPIE, 809914-809917 (2011). 
157. M. Gangishetty, K. Lee, R. Scott, and T. Kelly, "Plasmonic enhancement of dye sensitized solar cell in the red-to-near-infrared region using triangular core-shell Ag@SiO2 nanoparticles," ACS Appl Mater Interfaces 5, 11044 - 11051 (2013).

158. C. Wu, X. Zhou, and J. Wei, "Localized Surface Plasmon Resonance of Silver Nanotriangles Synthesized by a Versatile Solution Reaction," Nanoscale Research Letters 10, 354 (2015).

159. K. G. François, G. Mircea, P. Monica, M. N. Hounkonnou, and A. Simion, "Optical properties of single silver triangular nanoprism," Physica Scripta 86, 055702 (2012).

160. P. L. Geoffrey, B. Ashvin, A. H. Kimberly, M. Suraj, C. Yun-Sheng, and Y. E. Stanislav, "Silica-coated gold nanoplates as stable photoacoustic contrast agents for sentinel lymph node imaging," Nanotechnology 24, 455101 (2013).

161. R. Aniruddha, M. Ananya, X. Zhixing, K. Leshern, W. Xueding, and K. Raoul, "Highly stable polymer coated nano-clustered silver plates: a multimodal optical contrast agent for biomedical imaging," Nanotechnology 25, 445104 (2014).

162. F. J. van der Meer, D. J. Faber, D. M. B. Sassoon, M. C. Aalders, G. Pasterkamp, and T. G. van Leeuwen, "Localized measurement of optical attenuation coefficients of atherosclerotic plaque constituents by quantitative optical coherence tomography," Medical Imaging, IEEE Transactions on 24, 1369-1376 (2005).

163. A. Mariampillai, B. A. Standish, E. H. Moriyama, M. Khurana, N. R. Munce, M. K. K. Leung, J. Jiang, A. Cable, B. C. Wilson, I. A. Vitkin, and V. X. D. Yang, "Speckle variance detection of microvasculature using swept-source optical coherence tomography," Opt. Lett. 33, 1530-1532 (2008).

164. M. S. Mahmud, D. W. Cadotte, B. Vuong, C. Sun, T. W. H. Luk, A. Mariampillai, and V. X. D. Yang, "Review of speckle and phase variance optical coherence tomography to visualize microvascular networks," Journal of Biomedical Optics 18, 050901-050901 (2013).

165. P. B Johnson and R. W Christy, " Optical Constant of Noble Metals", Physical Review B, 6, 4370-4379 (1972)

166. C.-K. Lee, H.-Y. Tseng, C.-Y. Lee, S.-Y. Wu, T.-T. Chi, K.-M. Yang, H.-Y. E. Chou, M.-T. Tsai, J.-Y. Wang, Y.-W. Kiang, C.-P. Chiang, and C. C. Yang, "Characterizing the localized surface plasmon resonance behaviors of Au nanorings and tracking their diffusion in bio-tissue with optical coherence tomography," Biomedical Optics Express 1, 1060-1073 (2010).

167. G. M. Marco, "The physics of biofilms - an introduction," Journal of Physics D: Applied Physics 49, 203001 (2016). 
168. M. D. Rodney, "Biofilms and Device-Associated Infections," Emerging Infectious Disease journal 7, 277 (2001).

169. A. Dongari-Bagtzoglou, "Pathogenesis of mucosal biofilm infections: challenges and progress," Expert Review of Anti-infective Therapy 6, 201-208 (2008).

170. S. S. Magill, J. R. Edwards, W. Bamberg, Z. G. Beldavs, G. Dumyati, M. A. Kainer, R. Lynfield, M. Maloney, L. McAllister-Hollod, J. Nadle, S. M. Ray, D. L. Thompson, L. E. Wilson, and S. K. Fridkin, "Multistate Point-Prevalence Survey of Health CareAssociated Infections," New England Journal of Medicine 370, 1198-1208 (2014).

171. W. E. Stamm, "Catheter-associated urinary tract infections: Epidemiology, pathogenesis, and prevention," The American Journal of Medicine 91, S65-S71 (1991).

172. R. Podschun, and U. Ullmann, "Klebsiella spp. as Nosocomial Pathogens: Epidemiology, Taxonomy, Typing Methods, and Pathogenicity Factors," Clinical Microbiology Reviews 11, 589-603 (1998).

173. C. Vuotto, F. Longo, M. P. Balice, G. Donelli, and P. E. Varaldo, "Antibiotic Resistance Related to Biofilm Formation in Klebsiella pneumoniae," Pathogens 3, 743-758 (2014).

174. P. D. Lister, D. J. Wolter, and N. D. Hanson, "Antibacterial-Resistant Pseudomonas aeruginosa: Clinical Impact and Complex Regulation of Chromosomally Encoded Resistance Mechanisms," Clinical Microbiology Reviews 22, 582-610 (2009).

175. G. Sharma, S. Rao, A. Bansal, S. Dang, S. Gupta, and R. Gabrani, "Pseudomonas aeruginosa biofilm: Potential therapeutic targets," Biologicals 42, 1-7 (2014).

176. W. V. Sorin, and D. F. Gray, "Simultaneous thickness and group index measurement using optical low-coherence reflectometry," IEEE Photonics Technology Letters 4, 105107 (1992).

177. K. W. K. Lee, S. Periasamy, M. Mukherjee, C. Xie, S. Kjelleberg, and S. A. Rice, "Biofilm development and enhanced stress resistance of a model, mixed-species community biofilm," The ISME Journal 8, 894-907 (2014).

178. S. P. Dzul, M. M. Thornton, D. N. Hohne, E. J. Stewart, A. A. Shah, D. M. Bortz, M. J. Solomon, and J. G. Younger, "Contribution of the Klebsiella pneumoniae Capsule to Bacterial Aggregate and Biofilm Microstructures," Applied and Environmental Microbiology 77, 1777-1782 (2011).

179. S. A. Crusz, R. Popat, M. T. Rybtke, M. Cámara, M. Givskov, T. Tolker-Nielsen, S. P. Diggle, and P. Williams, "Bursting the bubble on bacterial biofilms: a flow cell methodology," Biofouling 28, 835-842 (2012). 
180. L. Wind, and W. W. Szymanski, "Quantification of scattering corrections to the BeerLambert law for transmittance measurements in turbid media," Meas Sci Technol 13 (2002).

181. J. Li, Z. Shen, Y. He, Z. Tu, Y. Xia, C. Chen, and S. Liu, "Quantitative measurement on optical attenuation coefficient of cell lines $5-8 \mathrm{~F}$ and $6-10 \mathrm{~B}$ using optical coherence tomography," Journal of Applied Physics 112, 084702 (2012).

182. H. Chen, X. Chen, Z. Qiu, D. Xiang, W. Chen, F. Shi, J. Zheng, W. Zhu, and M. Sonka, "Quantitative analysis of retinal layers' optical intensities on 3D optical coherence tomography for central retinal artery occlusion," Scientific Reports 5, 9269 (2015).

183. Thorlab Swept Laser Source SL1325 P-16 Catalog (https://www.thorlabs.com/catalogPages/608.pdf)

184. W. Georges, C. Shangguan, Z. Matthieu, U. Nora, B. Daniel, B. Jean-Pierre, and B. Hubert van den, "An optical phantom with tissue-like properties in the visible for use in PDT and fluorescence spectroscopy," Physics in Medicine and Biology 42, 1415 (1997).

185. D. M. de Bruin, R. H. Bremmer, V. M. Kodach, R. de Kinkelder, J. van Marle, T. G. van Leeuwen, and D. J. Faber, "Optical phantoms of varying geometry based on thin building blocks with controlled optical properties," Journal of Biomedical Optics 15, 025001-025001-025010 (2010).

186. C. Rinaldo, P. Antonio, T. Paola, T. Alessandro, and V. Gianluca, "A solid tissue phantom for photon migration studies," Physics in Medicine and Biology 42, 1971 (1997).

187. D. Wang, Y. Chen, and J. T. C. Liu, "A liquid optical phantom with tissue-like heterogeneities for confocal microscopy," Biomedical Optics Express 3, 3153-3160 (2012). 